\title{
Division of Energy Biosciences Annual Report and Summaries of FY 1996 Activities
}

\section{April 1997}

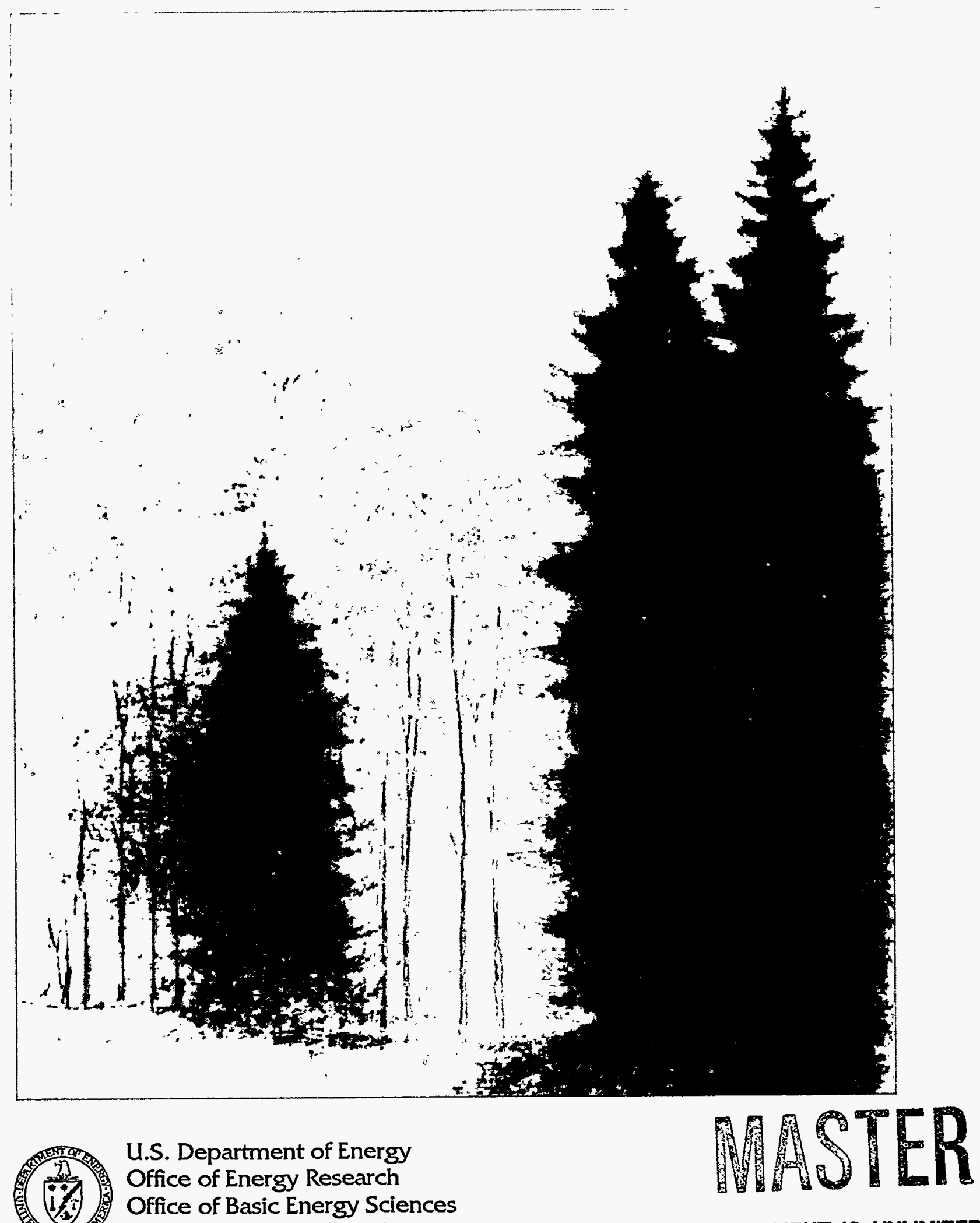

Office of Basic Energy Sciences

Division of Energy Biosciences DISTRIBUTION OF. THIS DOCUARENT IS UNLIMITED 
This report has been reproduced directly from the best available copy.

Available to DOE and DOE Contractors from the Office of Scientific and Technical Information, P.O. Box 62, Oak Ridge, TN 37831; prices available from (423) 576-8401.

Available to the public from the U.S. Department of Commerce, Technology Administration, National Technical Information Service, Springfield, VA 22161, (703) 487-4650.

Cover Picture: Skogsväg, an enlarged reproduction of a drypoint engraving by Gunnar Norrman of Lomma, Sweden. 


\section{DISCLAIMER}

This report was prepared as an account of work sponsored by an agency of the United States Government. Neither the United States Government nor any agency thereof, nor any of their employees, make any warranty, express or implied, or assumes any legal liability or responsibility for the accuracy, completeness, or usefulness of any information, apparatus, product, or process disclosed, or represents that its use would not infringe privately owned rights. Reference herein to any specific commercial product, process, or service by trade name, trademark, manufacturer, or otherwise does not necessarily constitute or imply its endorsement, recommendation, or favoring by the United States Government or any agency thereof. The views and opinions of authors expressed herein do not necessarily state or reflect those of the United States Government or any agency thereof. 


\section{DISCLAIMER}

Portions of this document may be illegible in electronic image products. Images are produced from the best available original document. 


\section{Program Overview of the Division of Energy Biosciences}

The mission of the Division of Energy Biosciences is to support research that advances the fundamental knowledge necessary for the future development of biotechnologies related to the Department of Energy's mission. The departmental civilian objectives include effective and efficient energy production, energy conservation, environmental restoration, and waste management. The Energy Biosciences program emphasizes research in the microbiological and plant sciences, as these understudied areas offer numerous scientific opportunities to dramatically influence environmentally sensible energy production and conservation. The research supported is focused on the basic mechanisms affecting plant productivity, conversion of biomass and other organic materials into fuels and chemicals by microbial systems, and the ability of biological systems to replace energy-intensive or pollutant-producing processes. The Division also addresses the increasing number of new opportunities arising at the interface of biology with other basic energy-related sciences such as biosynthesis of novel materials and the influence of soil organisms on geological processes.

The Division of Energy Biosciences supports research at the very fundamental level in an effort to support as broad a scientific foundation for the Department's technology development efforts as possible. The Department currently expends considerable effort through its Offices of Energy Efficiency and Renewable Energy, Fossil Energy, and Environmental Management to develop technologies based on available fundamental knowledge. The biological research supported by the Energy Biosciences program includes basic research on plants, algae and photosynthetic bacteria with emphases on photosynthetic mechanisms and bioenergetics, control of plant growth and development, genetic transmission and expression, and plant cell wall structure and function.

Support in these areas seeks to define and understand the biological mechanisms that effectively transduce light energy into chemical energy, to identify the biochemical pathways and genetic regulatory mechanisms for the energy efficient biosynthesis of potential fuels and petroleum-replacing compounds, and to elucidate the capacity of plants to remediate contaminated environments by transporting and detoxifying toxic substances. Another area covered by the Division is fermentative (and related) microbiology, which includes support of basic research on fungal and bacterial metabolism and physiology with emphases on anaerobic bacteria, bacteria that live in extremely 
harsh environments and biopolymer degradation by fungi and bacteria. Support in these areas seeks to provide the capability of converting organic and inorganic compounds, such as cellulose and sulfur-containing coal, in a rapid and energy efficient manner. Several DOE technology programs are interested in the potential to integrate biological-based systems into industrial processes.

The program's efforts in supporting interdisciplinary activities at the boundaries of biology and other disciplines represented within Basic Energy Sciences include: biocatalytic mechanisms, biomaterials and materials biosynthesis, interactions between plant/microbes with mineral particles, interface of photobiology and photochemistry, and bioengineering. The program also interacts extensively with other Federal agencies to coordinate and cooperate in areas of joint interest. The most prominent of these efforts has been the threeagency plant science activities in partnership with the National Science Foundation (NSF) and the U.S. Department of Agriculture (USDA). This partnership, initiated in 1987 under the auspices of the Office of Science and Technology Policy, now supports nine multi-institutional research coordinating group awards and eleven interdisciplinary research training group awards. The three agency programs have done much to stimulate plant science research nationwide on university campuses.

The Division of Energy Biosciences has joined with NSF and USDA to fund three national groups to initiate large scale sequencing of the genome of the plant Arabidopsis thaliana. These U.S. groups will coordinate with activities in Europe and Japan to decipher the entire genome of this model plant by the year 2004 . The three U.S. groups expect to sequence a minimum of $20 \%$ of the Arabidopsis genome within the first 3 years of the effort, and an equal amount of sequence is expected from the combined effort of 17 European laboratories and one Japanese laboratory during that period. The sequence of each clone will be released as it is completed and the user community can expect to see about 1 Mega base per month of annotated sequence entering the public databases.

\section{Recent Accomplishments}

Researchers in the Biomaterials Program at Lawrence Berkeley National Laboratory have been studying self-assembling thin-film biosensors that recently led to the development of a rapid and sensitive detector of the toxic Escherichia coli strains, which have caused outbreaks of deaths from contaminated foods. The fundamental design of the biosensor is the incorporation of a receptor-specific ligand into a long chain of linked lipid molecules that together constitute a polydiacetylene film, which happens to be blue. The binding of a specific ligand to the receptor molecule alters the linkage of the lipids in the polydiacetylene film, changing the color of the film 
from blue to reddish magenta. This biosensing litmus film can be developed with almost any receptor-ligand detector combination. In the biodetector film developed for $E$. coli, the human eye can easily see a color change triggered by $E$. coli concentrations in the parts-per-million range.

Research at Ohio State University has identified a signal transduction that is likely involved in integrating three central metabolic functions of photosynthetic bacteria. The three functions are photosynthetic energy capture, nitrogen fixation and carbon dioxide assimilation. The isolation of a single gene involved in the global regulation of three critical physiological processes is a major step in understanding the genetic regulatory machinery of bacteria.

During Fiscal Year 1996, the Division provided partial support for the following conferences, workshops or training activities:

1. FASEB Summer Research Conference: Signal Transduction in Plants, Copper Mountain, CO, June 16-21, 1996

2. Eighth International Symposium on Molecular Plant-Microbe Interactions, University of Tennessee, Knoxville, TN, July 14-19, 1996

3. FASEB Summer Research Conference on Plant Developmental Genetics, Saxton River, VT, August 10-15, 1996

4. Conference: The Art of Anaerobes, University of Georgia, Athens, $G A$, August 16-17, 1996

5. Workshop on Plant Growth, Lewes, DE, August 18-21, 1996

6. Investigations into the Metabolic Diversity of Microorganisms as Part of Microbial Diversity, Woods Hole, MA, Summer 1996

7. International Symposium Thermophiles 96 and International Workshop Thermophiles: Keys to Molecular Evolution and the Origin of Life?, University of Georgia, Athens, GA, September 4-12, 1996

8. Workshop on Microbes and Minerals: Mechanisms at the Interface, Pennsylvania State University, September 22-23, 1996

9. Diversity, Genetics and Physiology of Photosynthetic Prokaryotes, Indiana University, Bloomington, IN, October 18-20, 1996 
10. Genetics and Molecular Biology of Industrial Microorganisms (GMBIM-6): Augmented Access by Younger Faculty and Students, Indiana University, Bloomington, IN, October 20-24, 1996

11. An Advanced Course in Plant Biochemistry, Washington State University, Púllman, WA, to be held July 20-August 1, 1997

The Division continues to participate in the Life Sciences Research Foundation post-doctoral fellowship program, with three-year fellowship support for the following individuals in Fiscal Year 1996:

Dr. Paul Blount (Lab of Molecular Biology, University of Wisconsin, Madison)

Dr. David Lerner (Biology Dept., University of California, San Diego)

Dr. Peter Margolis (Dept. Biological Sciences, Stanford University, Stanford, CA)

Dr. Hank Bass (University of California, Berkeley)

Dr. Krishna Niyoga (Dept. Plant Biology, Carnegie Inst. of Washington, Stanford, CA)

Dr. David Weiss (Dept. Microbiology \& Molecular Genetics, Harvard Medical

School)

Dr. S.P. Dinesh-Kumar (University of California, Albany)

Dr. Andrew Millar (University of Virginia, Charlottesville)

Dr. Jacqueline Heard (Harvard Medical School)

Dr. Andrew Kloek (Washington University)

Dr. Jennifer Kuzma (The Rockefeller University)

Dr. Ramin Yadegari (University of California, Berkeley)

Each of the individuals is working in an area related to the Energy Biosciences program scope. 
The Division of Energy Biosciences was provided approximately thirty million dollars in fiscal year 1996. The breakdown of how the resources were distributed is indicated in the following table.

\begin{tabular}{|c|c|c|c|}
\hline & $\begin{array}{l}\text { Number } \\
\text { of } \\
\text { Projects }\end{array}$ & $\begin{array}{c}\text { FY } 96 \\
\text { funding } \\
\text { (in thousands) }\end{array}$ & $\begin{array}{l}\text { Percent } \\
\text { of } \\
\text { total } \\
\text { funds }\end{array}$ \\
\hline $\begin{array}{l}\text { University and Non-Profit } \\
\text { Institutions }\end{array}$ & 200 & 21,582 & 71 \\
\hline $\begin{array}{l}\text { Michigan State University } \\
\text { Plant Research Laboratory }\end{array}$ & 14 & 2,950 & 10 \\
\hline $\begin{array}{l}\text { National Laboratories } \\
\text { Brookhaven National Lab } \\
\text { Lawrence Berkeley Lab } \\
\text { Los Alamos National Lab } \\
\text { National Renewable Energy Lab. }\end{array}$ & 11 & 2,538 & 8 \\
\hline $\begin{array}{l}\text { Small Business Innovation } \\
\text { Research (SBIR) contribution, } \\
\text { and Miscellaneous }\end{array}$ & & 2,576 & 9 \\
\hline $\begin{array}{l}\text { Conferences, Educational } \\
\text { Activities }\end{array}$ & 12 & 429 & 1 \\
\hline Databases (joint funding) & 1 & 125 & $<1$ \\
\hline & 238 & $\$ 30,200$ & \\
\hline
\end{tabular}


The Energy Biosciences Division staff wish to thank the hundreds of reviewers, both in this country and abroad, who have contributed their time and effort to the peer review process of the program through mail reviews, panel meetings and site visit reviews. Without your assistance the program would be unable to maintain the same quality.

The staff members of the Energy Biosciences program are:
Dr. Gregory L. Dilworth, Director
Ms. Patricia A. Snyder
Dr. James E. Tavares
Ms. Mary Jo Martin

The mailing address:

Division of Energy Biosciences, ER-17

Office of Basic Energy Sciences

U.S. Department of Energy

19901 Germantown Road

Germantown, MD 20874-1290

Communication contacts for the EB program:

Phone: $\quad 301-903-2873$

Fax: 301-903-1003

E-mail: greg.dilworth@oer.doe.gov

james.tavares@oer.doe.gov

pat.snyder@oer.doe.gov

mary-jo.martin@oer.doe.gov

Web address: http://www.er.doe.gov/production/bes/eb/ebhome.html ; 


\title{
Abstracts of Projects Supported in FY 1996
}

\author{
U.S. Department of Agriculture \\ Madison, WI 53705-2398
}

1. Molecular Organization in the Native State of Wood Cell Walls: Studies of Tertiary Structure and its Development Using the Raman Microprobe, Solid State ${ }^{13} \mathrm{C}$ NMR, Fluorescence Spectroscopy and Photoconductivity R.H. Atalla, Forest Products Laboratory

$\$ 240,000$ (FY 95 funds/2 years)

Our studies of wood cell wall structure include both the variation of molecular organization within the walls and a search for deeper understanding of the phenomena of structure formation and their regulation by intracellular processes. We observe significant coupling between the aggregation of cellulose and the hemicelluloses, and clear evidence of the organization of the structure of lignin by the polysaccharide matrix within which it is polymerized. We believe that some unique characteristics of the secondary wall point to distinctive pathways in cell wall assembly that may not be obvious from studies of primary walls. We are, therefore, seeking a comprehensive paradigm for cell wall assembly that is applicable to both primary and secondary walls. We are guided by analysis of the organization of the constituents of woody tissue, at both cellular and sub-cellular levels, within the broader conceptual framework of hierarchic organization in biological systems. The methods we have used for characterization of woody tissues include Raman and Raman microprobe spectroscopy, solid state ${ }^{13} \mathrm{C}$ NMR, fluorescence spectroscopy and photoconductivity measurements. We complement these with theoretical modeling of intermolecular interactions and other traditional analytical procedures. The regularity of structure in adjacent cells leaves little doubt that the assembly of the wall is governed by intracellular processes that are not adequately accounted for in the accepted paradigms concerning the formation of the three major constituents of the cell wall.

\section{U.S. Department of Agriculture Beltsville, MD 20705-2350}

\section{Metabolic Regulation of the Plant Hormone Indole-3-acetic Acid} J.D. Cohen and J.P. Slovin, Beltsville Agricultural Research Center

The phytohormone indole-3-acetic acid (IAA, auxin) is important for many aspects of plant growth, development and responses to the environment. Our understanding of the biosynthesis of auxin in plants, and the mechanisms by which plants regulate auxin levels has changed remarkably within the last few years. We showed that mutant plants that cannot make the amino acid tryptophan still make IAA, and in very high amounts. We also showed that both the traditional tryptophan pathway and a non-tryptophan pathway to IAA occur in plants, and sometimes both are used by the same plant. An additional source of IAA in 
plants is the relatively large pool of IAA stored within plant cells in conjugated form. We are working on four fundamental problems related to how plants get their IAA: 1) Characterizing the non-tryptophan pathway to auxin using an in vitro system from maize seedlings; 2) Determination of how a plant decides to use one or the other pathway, or both, during various developmental stages or in response to environmental stresses and stimuli; 3) Isolation and characterization of bacterial and plant enzymes that hydrolyze conjugates that could be useful for altering IAA metabolism in specific plant tissues; and 4) Analysis of auxin turnover, i.e. the rate at which IAA is made and destroyed. Recent studies have shown that IAA levels are controlled, in part, by a feedback mechanism that is sensitive to the levels of IAA but not linked to the levels of active synthetic auxins that might be present.

\section{U.S. Department of Agriculture Raleigh, NC 27695-7631}

\section{Regulation of Sucrose-Phosphate Synthase and Other Cytosolic Proteins by Reversible Protein Phosphorylation \\ S.C. Huber, USDAVARS and Departments of Crop Science and Botany, NCSU}

Studies have been continued to characterize the control of enzymes of sucrose metabolism by reversible protein phosphorylation. Efforts have focused on sucrose-phosphate synthase (SPS), a key enzyme of sucrose biosynthesis, and sucrose synthase (SuSy), which is thought to function in sucrose cleavage in vivo. With SPS, work has focused on the activation of the enzyme that occurs when plant tissues are subjected to osmotic stress. Although not yet conclusive, our results are consistent with the idea that a unique site on the protein molecule becomes phosphorylated under stress conditions and thereby activates sucrose synthesis, even though the major regulatory phosphorylation site (Ser-158) is fully phosphorylated. In other words, the inhibition of SPS activity caused by phosphorylation of Ser-158 is at least partially overcome by phosphorylation of the "osmotic-stress site." We have tentatively identified the site as well as the protein kinase that might be involved in its phosphorylation, and we are attempting to determine what aspect of the kinase (if any) is affected by stress. Understanding this process may uncover new information about how plants cope with stress, and could provide new avenues to increase stress tolerance of crop plants. With SuSy, we have identified the single in vivo phosphorylation site of the maize SS2 protein as Ser- 15 . This site was also phosphorylated in vitro by an endogenous $\mathrm{Ca}^{2+}$-dependent protein kinase. Phosphorylation of SuSy in vitro activates the cleavage reaction, suggesting that phosphorylation may be of regulatory significance. The phosphorylation site and sequences surrounding it are strictly conserved among species suggesting that phosphorylation of SuSy may be widespread. Overall, the results strengthen the notion that both aspects of sucrose metabolism, i.e., synthesis and degradation, may be controlled by protein phosphorylation. 


\section{U.S. Department of Agriculture Ithaca, NY 14853}

4. Phytoremediation of Metal-Polluted Soils: Mechanisms of Heavy Metal Absorption, Translocation, Accumulation and Tolerance in Plants L.V. Kochian, U.S. Plant, Soil and Nutrition Laboratory

$\$ 182,000$ (FY 95 funds/18 months)

Recently, there has been a tremendous amount of research interest in the phytoremediation of heavy metal contaminated soils. This interest has been driven partly by the existence of hyperaccumulating plant species that are endemic to metalliferous soils and can accumulate high levels of heavy metals such as $\mathrm{Zn}, \mathrm{Cd}, \mathrm{Pb}, \mathrm{Cu}$, and $\mathrm{Ni}$ in the shoot. Probably the most well known hyperaccumulator is Thlaspi caerulescens, which is a member of the Brassicaceae family and a $\mathrm{Zn}$ (and $\mathrm{Cd}$ ) hyperaccumulator. The unique physiology of heavy metal transport and tolerance in Thlaspi caerulescens makes it a very interesting experimental system for basic research on plant mechanisms of metal hyperaccumulation. During the past year, we have been studying the physiology of heavy metal $\left(\mathrm{Zn}^{2+}\right)$ transport in $T$. caerulescens, in comparison with a related nonaccumulator species, Thlapsi arvense. We characterized a high affinity $\mathrm{Zn}^{2+}$ uptake system in the roots of both Thlaspi species and showed this system was significantly upregulated or stimulated in $T$. caerulescens. Additionally, $\mathrm{Zn}^{2+}$ translocation to the shoot was enhanced 10-20 fold in $T$. caerulescens. These results indicate that there is a complex alteration of zinc transport processes in $T$. caerulescens resulting in $\mathrm{Zn}$ hyperaccumulation. While enhanced influx into the root symplasm is a significant aspect of hyperaccumulation, it is likely that unloading into the xylem and plasma membrane and tonoplast $\mathrm{Zn}$ transport in leaf cells are also critical sites for $\mathrm{Zn}^{2+}$ hyperaccumulation. Currently, we are using biochemical, biophysical and molecular approaches to dissect out the different components of this transport pathway.

il

\section{Arizona State University}

Tempe, AZ 85287-1604

\section{Antenna Organization and Regulation in Green Photosynthetic Bacteria R.E. Blankenship, Department of Chemistry and Biochemistry

All photosynthetic organisms contain chlorophyll pigments that function as an antenna, absorbing light and transferring excitations to a photochemical reaction center where energy storage takes place by a series of chemical reactions. The green photosynthetic bacteria are characterized by large antenna complexes known as chlorosomes. The overall objective of this project is to determine the molecular organization of pigments in chlorosome antennas, as well as the mechanisms of excitation transfer and regulation of this unique antenna system. The chlorosome pigments are organized in vivo into pigment oligomers in which direct pigment-pigment interactions are of dominant importance. Time-resolved spectroscopy 
has given insight into the pathway and kinetics of excitation flow from the peripheral region of the chlorosome to the reaction center. Ultrafast absorbance measurements have indicated that the pigments are very strongly coupled, leading to subpicosecond energy transfer. Coherent oscillations are observed in the transient absorbance profiles, along with low frequency vibrations in the resonance Raman spectrum. Green sulfur bacteria contain a redox-activated quenching mechanism for control of energy transfer efficiency. The quenching effect may be a control mechanism that protects the cell from damage during conditions where light and oxygen are present simultaneously. X-ray structural studies are underway for the Fenna-Matthews-Olson bacteriochlorophyll a antenna protein from the green sulfur bacterium Chlorobium tepidum. This protein is an intermediate in the energy transfer pathway from chlorosome to reaction center.

\section{Arizona State University Tempe, AZ 85287-1601}

\section{Chlorophyll Binding and Biogenesis of Photosystem II W. Vermaas, Department of Botany and Center for the Study of Early Events in Photosynthesis

In photosynthetic systems, chlorophyll synthesis is tightly coupled with availability and/or biogenesis of chlorophyll-binding proteins. Free chlorophyll is toxic in the light and in the presence of oxygen as it gives rise to the formation of highly reactive singlet oxygen. In spite of the ubiquitous presence of chlorophyll in oxygenic photosynthetic systems, the in vivo mechanisms that couple the synthesis of chlorophyll and of chlorophyll-binding proteins are poorly understood. We address this problem using the transformable cyanobacterium Synechocystis 6803 of which the entire genomic sequence has been determined. Two questions receive particular attention: (1) Other than proteins directly associated with the two photosystems in cyanobacteria, which polypeptides may bind chlorophyll, and (2) How does coordinated synthesis of chlorophyll and chlorophyll-binding proteins in the photosystems occur? When searching the Synechocystis 6803 genome sequence for open reading frames (ORFs) coding for proteins with chlorophyll-binding motifs, four ORFs coding for small (7 kDa) proteins were found with significant homology to membrane-spanning regions of light-harvesting chlorophyll-binding proteins; another homologous region is a $3^{\prime}$ extension of the ferrochelatase gene in Synechocystis 6803. Cloning and deletion of these ORFs is in progress to determine the function of these ORFs. To address the second question, we monitor synthesis of photosystem II components as a function of chlorophyll synthesis using mutants lacking photosystem I and with chlorophyll biosynthesis brought under strict light control. Processing of the D1 protein of photosystem II to its active form does not occur efficiently in the early stages of chlorophyll biosynthesis, suggesting that chlorophyll aids proper integration of the D1 protein into the membrane. We are now creating mutants lacking chlorophyll synthesis altogether, and will follow transcription and translation of photosystem II components in these mutants to determine how the synthesis of chlorophyll and chlorophyll-binding proteins are related in this in vivo system. 


\section{University of Arizona \\ Tucson, AZ 85721}

\section{Polyol Functions in Stress Protection of Photosynthesis H.J. Bohnert and R.G. Jensen, Department of Biochemistry}

$\$ 100,000$

The modification of plant function is the focus of this project with the long-term goal of modifying biochemical pathways that confer increased whole-plant tolerance to drought and high salinity. We explore the role of increased polyol production and accumulation in cytosol and chloroplasts on tolerance. Previous results have clearly shown that the function of polyols is multi-factorial - polyols (and, likely, other accumulating metabolites) being involved in both osmotic adjustment through their mass action and in an unknown function that does not require high concentrations of polyols. The effect of polyols in osmotic adjustment has been measured in tobacco accumulating the methylated inositol, D-ononitol. Correlated with ononitol amount, transgenic tobacco show improved growth characteristics and development compared to control plants. Chloroplast expression of a mannitol dehydrogenase that leads to the accumulation of mannitol in tobacco chloroplasts provided evidence for mannitol to be an effective scavenger specifically of hydroxyl radicals. Generation of the highly reactive hydroxyl radical is measured by a reaction with phenylalanine that results in various isoforms of tyrosine. The protective effect of mannitol on hydroxyl radical scavenging has been shown in vitro, and in isolated chloroplasts, cells and in whole plants. Experiments with plants that include the expression and compartment-specific accumulation of several polyols are in progress.

\section{University of Arizona Tucson, AZ 85721}

\section{Role of Pectolytic Enzyme in the Programmed Release of Cells from the Root} Cap of Higher Plants

M.C. Hawes, Departments of Plant Pathology and Molecular and Cellular Biology

Roots of many species are programmed to produce and separate thousands of living cells, root "border" cells, from their root tips daily. The controlled release of living somatic cells into the external environment is unique among higher organisms, and its function is unknown. The objective of this work is to test the hypothesis that cell wall degrading enzymes in the root cap of pea play a role in cell wall degradation leading to border cell separation. Activities of a pectinmethylesterase (PME) and a polygalacturonase (PG) are correlated with deesterification of pectin in the root, with changes in cell wall $\mathrm{pH}$, and with border cell separation and release. A root cap expressed PME CDNA has been cloned, and shown to be unique among plant PMEs cloned to date. Its mRNA is induced within 5 min of inducing renewed border cell separation. Concomitant with PME induction is a holistic switch in gene 
expression in the root cap, including the activation of genes involved in carbohydrate synthesis and processing, as well as degradation. This transcriptional switch is followed within 15 min by a $400 \%$ increase in mitosis in the root cap meristem. New cells can be collected within one hour. The development of border cells is regulated by environmental as well as endogenous signals. Preliminary studies reveal that the endogenous signal that regulates border cell development is a heat stable molecule, larger than $25 \mathrm{kd}$, which functions as a repressor of mitosis in the root cap.

\section{University of Arizona Tucson, AZ 85721}

\section{Rhizosphere Association of the Nitrogen Fixing Bacterial Species Azotobacter paspali with the Tropical Grass Paspalum notatum: Specificity and Significance to Plant Nutrition \\ C. Kennedy, Department of Plant Pathology $\$ 142,500$ (FY 95 funds/18 months)}

Several nitrogen fixing bacteria are known to colonize the rhizosphere region of a variety of monocot plants, both cereals and grasses. None has yet been unequivocally demonstrated to transfer nutritionally significant amounts of fixed nitrogen to their associated partner plant. Evidence obtained in field experiments conducted several years ago in Brazil indicates that Azotobacterpaspali specifically colonizes the roots of the subtropical grass Paspalum notatum and transfers a small but significant amount of fixed $\mathrm{N}$ to plant tissues. Our aim is to study this bacterial-plant association in the laboratory and green house, starting with sterile plants inoculated with pure-culture wild type and Nif mutant strains, to try to establish Koch's postulates for the importance of $A$. paspali in supplying fixed $N$ to $P$. notatum. Plants generated from sterilized seedlings have been grown in plant growth medium with and without added fixed nitrogen compounds; stunting of growth was severe in the absence of fixed nitrogen while addition of about 0.75 micromolar ammonium nitrate allowed full growth. Additions of between 0 and 0.75 uM fixed $\mathrm{N}$ allowed some, but not full, growth of $P$. notatum. Inoculation experiments have begun to establish conditions for obtaining maximal growth enhancement of $P$. notatum by $A$. paspali. In addition, isolates of $A$. paspali obtained recently from the rhizosphere of $P$. notatum grown in Brazil showed high degrees of motility and chemotaxis towards some carbon sources. Specific attraction by chemotaxis of $A$. paspali by $P$. notatum roots will be tested. 


\section{University of Arizona \\ Tucson, AZ 85721}

\section{Regulation of DNA Endoreduplication in Maize Endosperm}

B.A. Larkins, Department of Plant Sciences

Endoreduplication of nuclear DNA frequently precedes periods of high levels of gene expression in plants and animals. This process is common in tissues with high metabolic activity, such as the developing endosperm of cereals and the cotyledons of dicot seeds. During endoreduplication, cells amplify their genome without chromatin condensation, segregation or cytokinesis, resulting in multiple, uniform copies of the nuclear DNA. Although this process is well documented and quantitated, the reason it occurs and the molecular basis of its regulation are unknown. Presumably, endoreduplication occurs as a consequence of altering the normal cell cycle: there is a block at $G 2$ and a disruption in the dependency of S-phase on the completion of mitosis. We have found that endoreduplicating maize endosperm cells contain an active, catalytic inhibitor that reduces activity of $p 34 c d c 2$, and that the induction of DNA synthesis is maintained by increasing the level and activity of S-phase-related protein kinases, as shown by their ability to interact with the human $\mathrm{E} 2 \mathrm{~F}$ and adenovirus E1A proteins. We have also found the onset of endoreduplication coincides with the phosphorylation of a 107-kD protein. The nature and function of this protein is unknown, but its size matches that of several well known proteins that regulate factors involved in DNA replication, including the $\mathrm{G} 1$ check point, retinoblastoma. Recent experiments have shown, however, that this protein is most likely not $\mathrm{Rb}$. We isolated a CDNA from maize endosperm whose predicted protein product $(\mathrm{ZmRB})$ shows homology to the "pocket" $A$ and $B$ domains of the $\mathrm{Rb}$ protein family. We found ZmRB behaves as a "pocket" protein based on its ability to specifically interact with oncoproteins encoded by DNA tumor viruses (E7, T-Ag, E1A). ZmRB can also interact in vitro and in vivo with the replication-associated protein, RepA, encoded by the wheat $d$ warf virus. The maize $\mathrm{Rb}$-related protein undergoes changes in level and phosphorylation state concomitant with endoreduplication, and it is phosphorylated in vitro by an S-phase kinase from endoreduplicating endosperm cells. Thus, several features of ZmRB suggests it behaves similarly to its mammalian homologue. A monocional antibody prepared against $Z \mathrm{mRB}$ failed to react with the 107-kD phosphoprotein that appears at the onset of endoreduplication, suggesting, therefore, that the latter is an alternative cell cycle regulatory protein. 


\section{University of Arizona Tucson, AZ 85721}

\section{Role of Zein Proteins in Structure and Assembly of Protein Bodies and Endosperm Texture B.A. Larkins, Department of Plant Sciences

The hardness, or texture, of the maize kernel is an important trait that influences grain quality during shipping and handling, as well as the wet and dry milling characteristics of the seed. The biochemical basis for kernel hardness is poorly understood, but it appears to be influenced by qualitative and quantitative changes in the synthesis of the endosperm storage proteins, or zeins, and the way in which these proteins form into protein bodies. To better understand the role of the various zein proteins in protein body formation and how this affects endosperm texture, we are characterizing mutations that alter the synthesis of several types of zeins: alpha (floury2 and De*B30), beta (transposon insertion mutants), and gamma (opaque15 and transposon insertion mutants). We are also studying the interactions of zein proteins during protein body formation by expressing the genes encoding them in transgenic tobacco endosperm. Tobacco plants that individually express alpha- and gamma-zein genes synthesize both proteins, but only the gamma-zein accumulates in the seed. However, if the two plants are crossed, both alpha- and gamma-zein are found in the mature seed. This appears to result from an interaction between these two proteins, as immunocytochemistry of seeds from the hybrid revealed that the alpha-zein is surrounded by gamma-zein in small protein bodies that appear similar to those found in maize endosperm. The results show that gamma-zein stabilizes alpha-zein, and it may provide the mechanism for alpha-zein retention in the ER. We have begun crossing these plants with others expressing the beta-zein gene, and experiments are in progress to investigate the interactions between the three proteins. In previous research, we isolated a gene encoding a $22-\mathrm{kD}$ alpha-zein protein that appears to be the basis of the floury 2 mutation. This gene contains a point mutation that converts the last amino acid of the signal peptide from alanine to valine. This seems to prevent cleavage of the signal peptide, causing the protein to become anchored to the ER membrane and ultimately leading to a reduction in zein synthesis and abnormal protein body development. Using in vitro translation experiments, we recently confirmed that the defective signal peptide causes the protein to remain associated with the ER membrane. Furthermore, conversion of the valine to an alanine led to normal processing of the signal peptide. To confirm that this gene is floury2, we transformed it into embryonic maize cells and regenerated fertile plants. Seeds produced from outcrosses of the primary transformants segregated 1:1 for starchy endosperm. The mutant seeds uniformly produce the aberrant $22-\mathrm{kD}$ zein protein, while the vitreous normal ones do not. The starchy seeds also show a significantly increased level of $\mathrm{BiP}$, which is also characteristic of the floury 2 mutant. These results provide genetic proof that the floury 2 mutation results from a defective $22-k D$ alpha-zein protein. 


\section{University of Arizona} Tucson, AZ 85721

\section{Molecular Characterization of the Role of a Calcium Channel in Plant Development \\ K.S. Schumaker, Department of Plant Sciences $\$ \$ 124,001$}

During development, plants convert physiological signals into specific growth responses. The pathway from these cues to a new developmental program involves mechanisms that transduce cellular energy and lead to biochemical and molecular changes in the cells. In several species of moss, addition of the hormone cytokinin to cells of the proper developmental stage causes an increase in intracellular calcium which stimulates a cascade of events leading to the formation of buds. Our research is examining the properties, expression and regulation of a calcium channel that is involved in this cytokinin induction of bud formation.

Our studies of calcium influx into moss protoplasts and binding of a calcium channel antagonist (azidopine) to moss plasma membranes have provided a biochemical and molecular characterization of this moss calcium channel. We have identified two proteins in the moss plasma membrane that bind specifically to azidopine, and studies are underway to isolate these putative channel proteins. Access to the channel will allow us to determine if differential channel expression is important formoss development. Our previous studies have suggested roles for cytokinin and changes in voltage in channel regulation. Recent studies suggest that heterotrimeric guanine nucleotide binding proteins may also affect channel activity, and that they do so in a cytoplasm-independent manner. To extend our studies of channel regulation to specific cell types, stages of development and specific regions of the cells, electrophysiological studies are underway to characterize channel activity. Understanding the properties, expression and regulation of the channel will help us determine the molecular mechanisms underlying calcium regulation and calcium's role in plant development.

\section{University of Arizona Tucson, AZ 85721} 13. Phytoalexin Detoxification Genes and Gene Products: Implication for the
Evolution of Host Specific Traits for Pathogenicity
H. VanEtten, Department of Plant Pathology $\$ 192,000$ (2 years)

The fungus Nectria haematococca has a wide host range but all isolates obtained from the wild that are pathogenic on pea contain genes (PDA) for the detoxification of the pea phytoalexin pisatin. The introduction of a PDA gene into a PDA- isolate and the mutation of PDA by transformation-mediated gene disruption have demonstrated that PDA affects the 
amount of disease produced on pea and that additional pea pathogenicity (PEP) genes are required for high virulence on this host. Nine PDA genes have been identified and most are located on small chromosomes (ca. 1.6 Megabases). Previous studies have demonstrated that one of these PDA bearing chromosomes while dispensable for growth in culture, is required for high virulence on pea because it carries PEP genes in addition to PDA. During the past year, it has been shown that the other PDA-bearing chromosomes or portion bearing PDA are dispensable. Furthermore, pathogenicity.tests on tomato and carrot show there was no significant difference in lesions produced by isolates with or without PDA-bearing chromosomes, suggesting that genes for pathogenicity on these hosts were located on chromosomes other than the PDA-bearing chromosomes. Several PDA-progeny from the same genetic background were tested for virulence on tomato and two low virulence isolates were identified, suggesting that different alleles for tomato pathogenicity genes exist in $N$. haematococca. These results support the hypothesis that the PDA-bearing dispensable chromosomes carry pea-specific virulence genes while other chromosomes carry genes for pathogenicity on other hosts.

\section{University of Arizona Tucson, AZ 85721}

\section{Role of HSP100. Proteins in Plant Stress Tolerance}

E. Vierling and S. Lindquist, Department of Biochemistry and The Howard Hughes Medical Institute, The University of Chicago

$\$ 150,009$ (FY 95 funds/18 months)

During high temperature stress plants express heat shock proteins (HSPs) which are hypothesized to protect them from heat damage. We are investigating whether Arabidopsis HSP101, a homologue of yeast HSP104, can be manipulated to increase plant thermotolerance. HSP104 is required for thermotolerance in yeast, and we have previously shown that Arabidopsis HSP101 will complement the thermotolerance defect of yeast HSP104 mutants. We have produced transgenic plants transformed with constructs designed to express antisense HSP101 controlled by either the CaMV 35S promoter or an Arabidopsis heat shock promoter, and other plants designed to overexpress HSP101 constitutively using the CaMV promoter. Transgene segregation and HSP101 levels are currently being assessed in these transformants. Suitable thermotolerance assays have been developed to assess the phenotype of the transgenic plants. Genomic clones encoding HSP101 have been isolated and promoter regions are being defined in order to construct GUS and GFP reporter genes to localize HSP101 expression within the plant. Antibodies specific to Arabidopsis HSP101 have been generated and used to explore expression of HSP101 during development and under other stress conditions, including ozone fumigation, UV-B, pathogen infection, and amino acid starvation. We find that HSP101 is developmentally regulated, accumulating during seed maturation and stored in the dry seed. Preliminary data suggest that ozone stress and pathogen infection may induce HSP101, which could be a secondary result of 
oxidative damage. Finally, initial efforts have been made to construct yeast strains suitable for a suppressor screen of HSP104 mutants, in order to define interacting proteins.

\section{Boston College \\ Chestnut Hill, MA 02167-3860}

\section{Osmoregulation in Methanogens \\ M.F. Roberts, Merkert Chemistry Center}

$\$ 95,073$

This project is aimed at understanding how methanogens deal with osmotic stress and to use these insights for increasing the salt tolerance of other cells. Present studies are focused in four different areas: (1) in vivo ${ }^{13} \mathrm{C}$-edited ${ }^{1} \mathrm{H}$ NMR studies of Methanococcus thermolithotrophicus and Methanohalophilus strain FDF1 using soluble ${ }^{13} \mathrm{C}$-labeled substrates for methanogenesis to monitor organic solute production, uptake, or loss upon alteration of external $\mathrm{NaCl},(2){ }^{39} \mathrm{~K}$ and ${ }^{23} \mathrm{Na}$ relaxation studies of these organisms to characterize environmental differences for ions in low and high $\mathrm{NaCl}$ cultures, (3) HPLC analysis of organic solutes secreted by the cells during growth and alterations in these solutes when medium $\mathrm{NaCl}$ changes, and (4) characterization of several key enzymes involved in the salt-sensitive accumulation of the osmolytes $N^{\varepsilon}$-acetyl- $\beta$-lysine and DIP, an unusual osmolyte that occurs in hyperthermophiles. The first of these provides a continuous assay of how the cells alter their intracellular solutes in response to different medium conditions. The second uses NMR relaxation times to quantify changes in macromolecule/ion interactions with altered external $\mathrm{NaCl}$ (this may be critical information since in vitro studies have indicated that $\mathrm{K}^{+}$ concentrations are linked to osmolyte accumulation). The third study is aimed at understanding whether solutes that have been secreted by cells can be reinternalized upon increasing external $\mathrm{NaCl}$; it should also provide information on mechanisms of osmolyte regulation. Lastly, the enzyme work should provide a molecular level view of two enzymes whose in vivo activity is regulated by changes in external $\mathrm{NaCl}$ (and temperature in the case of DIP).

\section{Boyce Thompson Institute for Plant Research, Inc. Ithaca, NY 14853-1801}

\section{Differential Regulation of Plastid mRNA Stability D.B. Stern}

Chloroplast gene expression is often regulated at the level of mRNA stability. This regulation is required for the coordinated synthesis and assembly of the electron-transport chain macromolecular complexes. RNA stability is governed by an interplay between RNA secondary structures, RNA-binding proteins, and ribonucleases. We have focused on the chloroplast petD gene, which encodes subunit IV of the cytochrome b6/f complex. Using in 
vitro biochemical methods, we have purified and characterized a protein of $41 \mathrm{kDa}$ that recognizes a specific, conserved sequence in the $3^{\prime}$ untranslated region of petD mRNA, just downstream of the stem-loop structure required for RNA stability. This protein also exhibits endoribonuclease activity. The way in which its two activities, RNA-binding and RNA-degradation, are regulated, is under study. Using tobacco chloroplast transformation, we have created plants where the petD gene is disrupted. These plants are being used to study the way in which chloroplast genes encoding several components of the cytochrome b6/f complex are regulated. For example, the petA gene encoding cytochrome $f$ may be dependent on the product of the petD gene for high-level expression. In addition, tobacco chloroplast transformation has been used to define the role of the $3^{\prime}$ stem-loop in higher plant chloroplasts. We have found that although an intact stem-loop is not required for expression of a reporter gene, it is required for accumulation of a discrete message. This is consistent with data we have published earlier for the chloroplast of Chlamydomonas reinhardtii, and thus allows us to generalize about the role of $3^{\prime}$ untranslated region secondary structures in chloroplast mRNAs.

\section{Brookhaven National Laboratory Upton, NY 11973}

\section{Molecular Plant Genetics \\ B. Burr and F.A. Burr, Biology Department}

$\$ 330,000$

Two recombinant inbred families have been used to construct a high density map of the maize genome. These populations are used by the maize genetics community for rapid mapping of cloned genes. The current map has over 1,900 markers. A database for these markers is now available on the World Wide Web. We are now working on the next generation of molecular markers based on simple sequence repeats (SSRs). Seventy-eight of these have been characterized and mapped. These loci are highly polymorphic and easy to assay. The SSRs will greatly speed up mapping in new populations and will facilitate the use of molecular markers in plant breeding.

We used the recombinant inbred populations to study the nature of genes that have quantitative rather than qualitative effects. Preliminary lessons are that regulatory genes or genes that control rate limiting steps are the most likely to be implicated in this type of inheritance. Current emphasis is on isolation and characterization of genes regulating two model systems--anthocyanin and carotenoid biosynthesis. We characterized in 1, a negative regulator of anthocyanin biosynthesis and are attempting to describe wc1, an apparent regulator of carotenoid biosynthesis. 


\title{
Brookhaven National Laboratory Upton, NY 11973
}

\author{
18. Molecular Bases of Photoadaptation in Unicellular, Eucaryotic Algae \\ P.G. Falkowski and J. LaRoche, Department of Applied Science \\ $\$ 84,000$
}

The goal of this research effort is to elucidate the molecular signal transduction pathways that lead to the irradiance-induced changes in the abundance and composition of photosynthetic proteins. Pharmacological studies with the unicellular chlorophyte alga, Dunaliella tertiolecta, have revealed that the redox state of the plastoquinone pool specifically has a marked effect on the expression of the chlorophyll $a / b$ binding proteins and their message levels. The redox state of this photosynthetic electron transport element is also known to induce protein phosphorylation. Gel mobility shift assays reveal the presence of high light induced DNA binding protein that is repressed by inhibiting reduction of plastoquinone. The protein binds to a region $5^{\prime}$ to the reading frame of the cab genes that has high sequence homology with light regulated elements in higher plants that are responsive to phosphorylated intermediates. Using transformation and biochemical approaches we are testing the hypothesis that the irradiance response is keyed to photosynthetic electron transport through a phosphorylation cascade which reversibly represses cab gene expression. Redox sensitive control of nuclear gene expression appears to be a general signal transduction pathway for a wide variety of environmental cues, including temperature, sugar accumulation and $\mathrm{CO}_{2}$ availability.

\section{Brookhaven National Laboratory Upton, NY 11973}

\section{Regulation of Energy Conversion in Photosynthesis}

The efficiency of photosystem II and the distribution of excitation energy from photosystem II to photosystem I are dynamically controlled by thylakoid-bound enzymes that modify pigments and pigment-protein complexes. Reversible protein phosphorylation on threonine residues is an important component in this regulation, and involves many photosystem II proteins. Evidence from mutant and inhibitor studies indicates that multiple protein kinases are involved.

We have previously described a $64-\mathrm{kDa}$ enzyme, which can be solubilized with octylglucoside and partially characterized. However, enzyme activity is consistently detected in the unsolubilized fraction. Established membrane-fractionation protocols were used to explore the origin of this residual activity, which remained in tight association with core complexes containing photosystem II reaction centers. An entirely novel $58-\mathrm{kDa}$ protein kinase was identified in core complexes by renaturating a Western blot and probing for autophosphorylation and histone-phosphorylation activities. This enzyme catalyzes 
phosphorylation of the intrinsic CP43 component of photosystem II cores, and light-harvesting chlorophyll $a / b$ protein supplied exogenously. Future studies will explore the substrate preferences of the 64- and 58-kDa kinases, and possible interaction between these enzymes. Bulk fractionation of core complexes toward obtaining 58-kDa kinase for sequencing and cloning is in progress. We also are studying the mechanism of redox regulation of these enzymes.

Polyphenol oxidase (PPO) is released from thylakoids by octylglucoside. Analysis of the PPO primary sequence identified two highly conserved motifs which share homology with mammalian metalloproteinases. This indication of bifunctionality and the suggestion that PPO may participate in protein turnover, are being investigated.

\section{Brookhaven National Laboratory Upton, NY 11973}

\section{Modification of Plant Lipids \\ J. Shanklin, Biology Department}

$\$ 315,000$

Despite its central role in lipid metabolism for both structural and storage lipids, the process of fatty acid desaturation is poorly understood. We are using the soluble plant fatty acid desaturase system as a model to understand this process because it can be highly expressed in bacteria, and the purified protein is active and highly stable. We are using an interdisciplinary approach to define structure function relationships of various regions of the protein using molecular-genetic biochemical and $x$-ray crystallographic techniques. Using this integrated approach we hope to re-design the desaturase enzymes for desired chain length and double bond positional specificity. In the long term, such rationally designed enzymes will be introduced into crop plants to produce valuable added oils.

We are also attempting to define structure-function relationships in the other, and predominant, class of fatty acid desaturases and related hydroxylases, that are integral membrane proteins. A similar molecular-genetic biochemical approach in conjunction with various spectroscopic techniques is being used to define the active site for these enzymes.

\section{Brown University \\ Providence, RI 02912}

\section{1. $\delta$-Aminolevulinate Biosynthesis in Oxygenic Prokaryotes \\ S. Beale, Division of Biology and Medicine $\$ 210,000$ (FY 95 funds/2 years)}

Tetrapyrrole pigments function as essential components in the processes of respiration and photosynthesis, and also exist as cofactors in other important enzyme reactions. The 
tetrapyrroles arise from a branched biosynthetic pathway having $\delta$-aminolevulinic acid (ALA) as its first committed member. ALA is known to be formed by two distinct routes: by condensation of glycine and succinyl-CoA in animal, fungal, and some bacterial cells, and by transformation of the intact carbon skeleton of glutamate in plants, algae and other bacterial cells. It has become apparent that the five-carbon pathway from glutamate is more widely distributed than was previously thought, and it may be the usual route of ALA formation in phototrophic organisms in addition to being the universal mode in oxygenic species. The macromolecular components of the five-carbon ALA-forming system have been fractionated into three enzymes and a required tRNA component. Cell-free preparations have been obtained in our laboratory from several phototrophic prokaryotes, including oxygenic cyanobacteria and strict anaerobes, that catalyze ALA formation from glutamate by reactions similar to those occurring in plants and algae. We propose to continue the characterization of the enzymes and RNA reaction components derived from phototrophic prokaryotes and from the unicellular alga, Chlamydomonas reinhardtii, to compare them to their counterparts in higher plants, and to study the regulation of their activity in response to light and nutritional status. The potential of these organisms for molecular genetic studies will be exploited by cloning and sequencing the genes encoding enzymes that catalyze steps of tetrapyrrole biosynthesis. The cloned genes will be used to generate probes to study the regulation of their expression during adaptation of the cells to light and nutritional status. The probes will also be evaluated for use in measuring expression of homologous genes in other algae and higher plants.

\section{California Institute of Technology Pasadena, CA 91125}

\section{Molecular and Genetic Analysis of LEAFY, a Gene Controlling Floral Inductionand Flower Development in Arabidopsis thaliana

We are following two separate lines of research. One has the goal of understanding the mechanism of ethylene action in Arabidopsis thaliana, and the other the goal of learning the mechanism by which the LEAFY gene of Arabidopsis regulates flowering. The ethylene work follows upon our cloning of a family of genes that code for ethylene receptors, by the criteria of mutant phenotype and ethylene binding. We are completing the characterization of this gene family, which at present has five members. We have complete sequence data on three members, and have shown that four of them can be mutated to give ethylene insensitive plants. The mutations are genetically dominant. We are also isolating intragenic suppressors of the dominant mutations, to see what might be the loss of function phenotype of each receptor. The studies of floral induction are directed toward finding genes and proteins that interact with LEAFY, a key regulator of floral development. LFY activity is both necessary and sufficient for complete floral induction. 
We have found a number of loci that interact with LFY as genetic enhancers and suppressors of Ify mutants. Among them are genes such as APETALA1 and UNUSUAL FLORAL ORGANS, that act along with LFY to convert vegetative meristems to floral meristems; and also several of the known genes whose mutations cause late flowering. Detailed genetic and molecular analyses of LFY and of the genes with which it interacts are in progress.

\section{University of California Berkeley, CA 94720}

\section{The Molecular Genetics of Ligule Induction M. Freeling, Department of Plant Biology}

There has been considerable progress in understanding how the maize ligule and auricle are induced, positioned and formed. Two recessive mutants, $\lg 1$ and $\lg 2$, show deficient ligules and auricles and the genes have been cloned. The deduced amino acid sequences for both LG1 and LG2 are now complete (Moreno et al., 1996, submitted, Walsh, unpubl.). Obvious transcription factor homologies are evident: squamosa promoter binding protein and ocs-type basic leucine zipper, respectively. Further, we have genetic evidence that LG2 acts cell-nonautonomously and that LG1 acts cell-autonomously and both act synergistically, at least after ligule induction (Harper and Freeling, Genetics, 1996). These data lead us to the hypothesis that LG1 and LG2 work together in the preligular primordial region to draw a line, on which the ligule will be induced. If no line is specified, no ligule can be formed.

Expression of a mutant VG1 product kills the ligule and some marginal sheath after it has been formed. This cell death involves DNA fragmentation, much like apoptosis in animals (Jesaitis and Kaplinsky, unpubl.).

\section{University of California Berkeley, CA 94720}

\section{Cellular Integration of MVA Synthesis and Protein Prenylation W. Gruissem, Department of Plant Biology}

The multi-branched isoprenoid pathway from mevalonic acid (MVA) is one of the most important pathways in plants because isoprenes are required for many cellular processes such as cell division and growth, defense, hormone signaling, and photosynthesis. Research in animals and yeast has established MVA synthesis and prenylation of growth-related signal transduction proteins as critical factors for cell cycle progression and normal cell growth. MVA synthesis is also critical during plant development, but it is unknown how isoprenoid production is coordinated with cell division and growth. Modification of select regulatory proteins by farnesyl of geranylgeranyl may provide an important link between the isoprenoid 
pathway and growth control. Protein prenyltransferases have been found in all eukaryotic cells. The plant farnesyltransferase can fully complement a mutation in the yeast enzyme for restoration of the Ras signaling pathway, demonstrating that the function of this enzyme has been conserved during evolution. This project takes a combined biochemical, molecular and genetic approach to investigate MVA synthesis and protein prenyltransferases in plants and to determine their regulation during development and cell cycle progression. As most targets of protein prenyltransferases are currently unknown in plants, the cloned enzymes will be important tools in their identification. Together, the experiments will establish necessary tools and the basis to understand the cellular network that integrates early steps in the isoprenoid biosynthesis pathway with proteins that provide structural and regulatory functions during cell growth.

\section{University of California Berkeley, CA 94720}

\section{Determinants of Environmental Stress Tolerance by Bacteria on Leaves S.E. Lindow, Department of Plant and Microbial Biology} $\$ 84,000$ (FY 95 funds/2 years)

Bacteria that live as epiphytes on the surface of healthy plants are important as plant pathogens, in causing plant frost injury by catalyzing ice formation, and in other processes. The objectives of this study are to determine the traits of these epiphytic bacteria that allow them to survive the stress of the hostile leaf surface environment. We have applied a random mutagenesis approach to identify genes in Pseudomonas syringae that are involved in epiphytic fitness, thereby directly identifying genes which are required for epiphytic growth or survival, and avoiding the limitations of investigating only predetermined phenotypes exhibited by bacteria in culture. Four transposon mutants showing different patterns of reduced growth and survival during physical stresses on leaves in the greenhouse and in the field have been biologically characterized; the distinctive behaviors of these mutants suggest that several loci are involved in stress tolerance. One locus, estA is constitutively expressed and appears to be required for methionine biosynthesis that is apparently necessary for responses to rapid changes in the cell environment. The sequences of the loci interrupted in two other mutants do not show similarity to any other cloned genes. We are pursuing the hypothesis that these loci regulate other genes which individually confer only partial ability to survive environmental stresses on leaves, since these mutants are pleiotropic, having altered tolerance of water stresses, extracellular polysaccharide production, and changes in other traits besides epiphytic fitness. 


\section{University of California Berkeley, CA 94720}

\section{Phylogenetic Analysis of Hyperthermophilic Natural Populations Using Ribosomal RNA Sequences \\ N.R. Pace, Department of Plant and Microbial Biology \\ $\$ 77,000^{*}$}

Hyperthermophilic organisms (growth optima $>80^{\circ} \mathrm{C}$ ) are a largely unexplored pool of biota with substantial potential for biotechnology. We know little about the diversity of life at high temperatures because of general reliance upon establishing laboratory cultures for assessment of organisms. It is well-known, however, that only a minor fraction of naturally occurring microorganisms is routinely culturable. The main goal of the program is, therefore, to survey phylogenetically and quantitatively the microbial biodiversity in selected high-temperature ecosystems using methods that do not rely upon cultivation. Phylogenetic information allows inference of some properties of organisms, and the sequences provide for subsequent identification of organisms and the design of probes for further studies.

$16 S$ rRNA genes in DNA isolated from high-temperature environmental samples are isolated by cloning, directly or following amplification by polymerase chain reaction, for sequence and phylogenetic analysis. Hybridization probes prepared from recombinant $16 S$ rRNA genes are used to evaluate the quantitative representation of particular sequence representatives and to identify the corresponding organism in environmental samples. Environments include diverse Yellowstone settings and deep-sea hydrothermal vents. Many novel hyperthermophiles have already been discovered using this approach, including new and most deeply divergent lineages of Bacteria and Archaea.

The program uses existing methods and continues to develop new ones for rapid analysis of natural communities. Additionally, $16 \mathrm{~S}$ rRNA sequence and phylogenetic analyses are carried out for selected hyperthermophilic organisms already in culture. These sequences make possible phylogenetic analysis of the organisms from which the sequences derive and contribute to the data base to which natural populations are compared. The sequences also are used for comparative analyses of hyperthermophilic and related mesophilic 16S rRNAs, to identify primary and secondary structural elements that may contribute to extreme thermostability.

*Funded collaboratively with DOE's Office of Health and Environmental Research 


\section{University of California} Berkeley, CA 94720

\section{Phytochrome from Green Plants: Properties and Biological Function P.H. Quail, Department of Plant and Microbial Biology \\ $\$ 105,000$}

The phytochrome (phy) family of informational photoreceptors has five members in Arabidopsis, phyA through phyE. In fully green seedlings, phyB is the predominant phytochrome with a major role in detection of competing neighboring plants. To identify regions and individual amino acids in the molecule important for its biological activity, we generated a series of mutant derivatives of phyB by random or site-directed mutagenesis and assayed for in-vivo activity in transgenic Arabidopsis. The data indicate that neither of the two major structural domains is alone sufficient, but rather both are contiguously necessary for activity. Small deletions at the amino terminus or in the central region compromised activity, indicating the importance of these regions. Point mutations which reduced phyB regulatory activity without affecting its levels or photoactivity were found to cluster in a 62-residue stretch in the central region of the molecule. The data indicate that the mutant molecules are fully active in photosignal perception, but defective in the regulatory activity responsible for signal transfer to downstream transduction pathway components. Together with data from domain-swap experiments and similar mutational analysis of phyA, the accumulating evidence suggests that the central region identified in these studies is a critical determinant in the regulatory activities of both phyA and phyB, possibly involving a common biochemical mechanism of primary signal transduction.

\section{University of California \\ Berkeley, CA 94720-3102}

\section{Molecular Cloning and Characterization of the Arabidopsis thaliana RPS2 Disease Resistance Locus \\ B.J. Staskawicz, Department of Plant Biology \\ $\$ 109,000$}

During the past year we have continued to develop Arabidopsis thaliana as a model host-pathogen system to study the molecular basis of pathogen recognition and the expression of plant disease resistance to bacterial pathogens. Our research has concentrated on genes from both the pathogen (avrRpt2) and the host (RPS2) that specify disease resistance in this gene-for-gene interaction. We have made considerable progress on studying the molecular events involved in the delivery of the avrRpt2 avirulence signal. The avrRpt2 gene encodes a putative $\mathbf{2 8 . 2}$ kilodalton protein that may be directly delivered into plant cells via the hrp secretory apparatus. To test this hypothesis, we have begun to construct epitope-tagged avrRpt2 avirulence genes. 'To date, we have successfully constructed c-terminal extensions of this gene with both the $6 \mathrm{x}$-His tag and a $1 \mathrm{xc}$ c-myc tag. The addition of these amino acids do not affect the delivery of the avirulence signal as both 
constructs maintain the ability to induce a race-specific HR on Arabidopsis plants containing the RPS2 resistance gene. Our future experiments will employ these constructs to conclusively demonstrate that the AvrRpt2 protein is delivered to the plant cell. Finally, we have also constructed an epitope-tagged RPS2 gene with the c-myc epitope. We plan to use this construct to determine the subcellular location of this protein and to use this construct in immunoprecipitation experiments to look for interacting proteins.

\section{University of California Berkeley, CA 94720}

\section{Analysis of Genes Essential for Floral Development in Arabidopsis P. Zambryski, Department of Plant Biology

Our continuing studies of the TOUSLED protein kinase gene have been in two main areas: 1) further characterization of its genetic interaction with genes important in flower morphogenesis, and 2) biochemical analysis of its enzymatic activity. Regarding the latter, we have purified the TSL protein expressed as a fusion protein from yeast, and demonstrated that it can autophosphorylate as well as trans phosphorylate several substrates. We have shown that the $\mathrm{N}$-terminus is critical for activity of the $\mathrm{C}$-terminal kinase domain, and that the protein likely functions as a dimer. Regarding our studies on the ETTIN gene, we have isolated the gene and determined that it encodes a protein with no homology to known sequences. ETT transcript is found at the earliest stages of flower development in the center of the floral meristem. ETT expression is then found in several different emerging organs (petals and stamen primordia as well as the center of the meristem before the gynoecium arises). ETT expression at several different times and places suggests that it has several different roles. That it most dramatically affects gynoecium morphology suggests it has a role in regional specification of the gynoecium. That it affects the number of petals and sepals suggests that it has a different role in the outer whorls of the flower, for example, to determine the pattern of organ primordia. Double mutant analysis with other mutants in the floral pathway support that ETT has multiple roles in floral development.

\section{University of California Davis, CA 95616}

\section{Biosynthesis of Cellulose and Callose in Developing Cotton Fibers D.P. Delmer, Section of Plant Biology $\$ 105,000$ (15.5 months)}

The ultimate goal of this project is to elucidate the mechanism and regulation of synthesis of two important beta-glucan polymers in plants--the cell wall polymer cellulose (beta-1,4-glucan) and the wound polymer callose (beta-1,3-glucan). In vitro synthesis of cellulose in plants is difficult, although callose synthase is easily demonstrable, and suggestions have been made 
that the cellulose and callose synthases share some common subunits. Characterization of two cDNA clones from cotton and one from rice that encode homologs of the catalytic subunit of the bacterial cellulose synthases has opened new approaches for the study of these processes. These genes are highly-expressed at the time of secondary wall cellulose synthesis in the cotton fiber, have highly-conserved domains that are proposed to be involved in substrate binding and catalysis, and recombinant protein does indeed bind the substrate UDP-glc. Future goals include the following: 1. To gain more proof that these CelA genes function in vivo in cellulose synthesis; approaches include further studies of gene expression in tissues that show varying rates of cellulose synthesis in vivo; anti-sense experiments with the CelA genes to observe effects on cellulose synthesis in vivo; and use of PCR-based approaches to screen for plant mutants that have a transposable element inserted in a CelA gene and to study the phenotypes of such plants; 2 . To study the localization and structure of CelA gene products in the plasma membrane of cotton, and to use the yeast two-hybrid method to select for genes that encode other polypeptides that might interact with CelA proteins in vivo. Likely candidates for interaction may be: CelA interacting with itself to form a multi-subunit synthase; sucrose synthase that we propose channels substrate to the synthase complex; the catalytic subunit of callose synthase and/or proteins that interact with callose synthase such as annexins and/or calnexin; or, components of the cytoskeleton that may interact to guide synthase movement.

\section{University of California Davis, CA 95616}

\section{Cellulose Binding Proteins of Clostridium cellulovorans Cellulase R.H. Doi, Section of Molecular and Cellular Biology $\$ 115,000$}

Our interest is to determine the structure and function of the Clostridium cellulovorans cellulosome, a complex enzyme containing about 10 different subunits and capable of digesting crystalline cellulose. The cellulosome consists of three major subunits and a number of minor subunits. We have characterized two of the major subunits, $\mathrm{CbpA}$ and ExgS. CbpA is a large (189 kDa) non-enzymatic subunit which acts as a scaffolding protein to which the enzymatic subunits bind. We have cloned and sequenced exgs which codes for ExgS, an exoglucanase. We are in the process of cloning and sequencing P100, the third major subunit, which appears to be an endoglucanase. CbpA contains 9 binding sites (EBDs) for enzymatic subunits and one cellulose binding. domain (CBD). We have shown that enzymatic subunits such as ExgS can bind strongly to the EBD of CbpA through a conserved duplicated sequence (DS) consisting of two 22 amino acid long sequences and more weakly through hydrophobic region(s) of the enzymatic subunit. The nine EBDs are quite similar in sequence but binding studies to ExgS indicate that certain EBDs can bind ExgS, whereas others cannot thus indicating that slight sequence differences in EBDs affect their ability to bind enzymatic subunits. By deletion studies we have shown that a mini-cellulosome can be constructed by mixing a mini-CbpA containing only the CBD and one-two EBD domains, ExgS 
and P100. Thus we are in a position to study the assembly and activity of a model mini-cellulosome. Further studies will include an analysis of the expression of the genes for the cellulosome subunits by using the isolated genes as probes during Northern blots.

\section{University of California \\ Davis, CA 95616-8627}

\section{Plant Physiological Aspects of Silicon \\ E. Epstein, T.W.-M. Fan, W.K. Silk, Department of Land, Air and Water Resources, and R.M. Higashi, Bodega Marine Laboratory $\$ \$ 98,968$}

The element silicon is second only to oxygen in its abundance in soils. It is readily absorbed by plants so that terrestrial plants contain amounts of it comparable to those of such major plant nutrients as calcium, magnesium, sulfur and phosphorus. Yet not being considered essential for plant growth and development, silicon is not included in the formulation of any of the conventional culture solutions widely used in plant physiological research. We have shown in this project that when wheat and rice plants are grown in solution cultures to which silicon was added, they rapidly absorb the element and as a result differ significantly from the silicon deprived plants grown in conventional (minus silicon) culture solutions. The differences relate to (i) inorganic plant nutrition; (ii) cell wall biochemistry; but above all (iii) the structural strength of plant cell walls, which is demonstrably greater in the silicon replete plants. As a result the disposition of leaves for photosynthetic energy capture is more favorable when silicon is available to wheat and rice plants. Tomato and cotton plants absorb much less silicon than wheat and rice plants, and as a result are much less affected by the silicon status of their substrate. Our research leads to the conclusion that silicon is much more significant for the normal growth, development, and energy economy of at least some plants than might be thought on the basis of experiments with conventional, i.e. minus silicon, solution cultures.

\section{University of California Davis, CA 95616}

\section{Regulation of Embryonic Development in Higher Plants J.J. Harada, Section of Plant Biology

The Arabidopsis LEAFY COTYLEDON1 (LEC1) gene plays a central role in integrating several diverse aspects of embryogenesis and postgerminative growth. LEC1 gene activity is required to inhibit the germination of developing embryos, to allow embryos to become desiccation tolerant, to initiate and/or maintain the expression of a subset of seed protein genes during embryogenesis, and to specify cotyledon identity, in part, by suppressing trichome formation on embryonic cotyledons. Although the effects of the lec1 mutation are most conspicuous during late embryogenesis, we have shown recently that the gene functions 
during early embryogenesis. Embryos of lec1 mutants and the other leafy cotyledon-type mutants, lec2 and fusca3 (fus3), undergo abnormal suspensor cell proliferation as early as the globular stage. lec1 fus 3 and lec1 aba insensitive 3 double mutants often give rise to secondary and tertiary embryos in the seed as a result of the enhancement of the suspensor abnormalities. Thus, LEC1 is also needed to suppress the embryogenic potential of the suspensor early in embryogenesis. A major objective has been to obtain a mechanistic understanding of LEC1 gene function. Exploiting a mutation generated by a T-DNA insertion, we have recently identified a region of DNA that is disrupted in both LEC1 mutant alleles. We have shown that this region contains a gene that is expressed embryo-specifically in wild type plants but that is not expressed in lec1 mutant embryos. Analysis of the LEC1 gene and its activity should provide substantial insight into its roles in embryogenesis.

\section{University of California Davis, CA 95616}

\section{Cellular and Molecular Characterization of Vascular Plasmodesmata W.J. Lucas, Section of Plant Biology \\ $\$ 119,000$}

Higher plants must integrate physiological and developmental processes occurring at different locations on the plant. Partitioning of photosynthate between these various plant organs is greatly influenced by environmental input signals and must involve a sophisticated endogenous control system capable of integrating photosynthate production, within the mesophyll tissue of the leaf, and phloem long-distance transport within the body of the plant. Recently, we established that plant function as supracellular organisms, in that plasmodesmata potentiate cell-to-cell trafficking of information molecules. In view of this finding, our attention has been focussed on testing the hypothesis that macromolecular transport, through plasmodesmata, acts as an important facet of the endogenous control network responsible for partitioning carbon and nitrogen resources at the whole plant level. Proteins extracted from the phloem of a number of plants have been used in microinjection experiments. In addition, genes encoding phloem sap proteins were expressed in $E$. coli and then fluorescently labeled for use in microinjection experiments. Collectively, these studies established that a large number of phloem proteins, ranging in molecular weight from 10 to $100 \mathrm{kDa}$, have the capacity to interact with plasmodesmata to both increase molecular size exclusion limit and mediate in their transport from cell to cell. Molecular studies are also being performed on selected phloem sap proteins to elucidate the mechanism(s) by which they move from the companion cell into the functional sieve element. This information will provide the foundation for future studies aimed at understanding the mechanism by which the plant integrates physiological and developmental processes at the whole plant level. 


\section{University of California Davis, CA 95616}

\section{Protein Translocation and Assembly in Chloroplasts S.M. Theg, Section of Plant Biology}

$\$ 98,000$

The objective of this project is to investigate a number of aspects relating to the biogenesis and operation of the oxygen-evolving complex (OEC) in chloroplasts. This enzyme complex, which is responsible for most of the oxygen in the atmosphere, is extrinsically associated with the inner surface of the chloroplast thylakoid membrane, and is formed from at least three nuclear-encoded subunits. These subunits must be translocated across the two chloroplast envelopes and the thylakoid membrane before they can be assembled into the enzyme complex. The experiments undertaken during this project are designed to elucidate the mechanisms that lead to complex formation and enzymatic activity. Experiments monitoring the assembly of the subunits into the complex after their in vitro import into isolated chloroplasts have revealed that membrane transport and assembly are coupled for one of the subunits. The nature of this coupling, and its implications for homeostasis of the complex are currently under investigation. In addition, we are investigating the environment through which the subunits pass as they traverse the thylakoid membrane. Specifically, we are monitoring the electrical conductivity of the thylakoid membrane during translocation to determine whether proteins are translocated through ion-permeable, aqueous pores.

\section{University of California Davis, CA 95616-8515}

\section{Vacuole Biogenesis in Differentiating Plant Cells T.A. Wilkins, Department of Agronomy and Range Science}

By tracing the regulation of vacuolar $\mathrm{H}^{+}$-ATPases in developing cotton seed trichomes, we have divided cell expansion into discrete substages related to changes in vacuolar function. The onset of expansion is accompanied by the biogenesis of a large central vacuole formed from two pre-existing vacuole populations and the prolific de novo synthesis of ER-derived provacuoles studded with fully assembled V-ATPase holoenzymes. The next substage of cell expansion, characterized by a period of very rapid polar elongation, can be detected in the vacuole by a sudden shift in the abundance and molecular weight of tonoplast proteins, including a dramatic increase in V-ATPase enzymatic activity. The increase in V-ATPase subunits is also associated with the coordinate induction of V-ATPase gene expression and increased enzymatic activity, indicating that V-ATPases are subject to multiple levels of regulation during cell expansion. Further analysis revealed that the two multigene families encoding the V-ATPase catalytic subunit A are differentially expressed during cell expansion, suggesting that the members of at least one gene family encode isoforms that are specifically induced during rapid cell expansion. These results suggest that $V$-ATPase isoforms are more 
likely associated with environmental, developmental and physiological responses than with the expression of tissue- and/or cell-specific genes. In a model proposed to depict the changes in vacuolar activity and function during cell expansion, the change in composition of the tonoplast during termination of cell expansion is believed to represent, at least in part, the selective removal and turnover of "expansion-associated" V-ATPase isoforms.

\section{University of California} Irvine, CA 92697

\section{Membrane Bioenergetics of Salt Tolerant Microorganisms J.K. Lanyi, Department of Physiology and Biophysics}

The energy costs of salt tolerance in the extremely halophilic bacteria originate from the generation of electrochemical ion gradients across the cytoplasmic membrane that drive sodium extrusion and chloride accumulation. We study the molecular mechanisms of the proton and chloride transporting bacterial rhodopsins and the proton transporting ATPase in these organisms. Studies of bacteriorhodopsin and halorhodopsin, respectively, explore the thermodynamics of the transport, the chromophore and protein changes that determine the changing connectivity of the active site to the two membrane surfaces during the transport cycle, and the mechanisms of ion conduction to and from the retinal Schiff base. Site-specific mutagenesis, time-resolved optical multichannel and infrared spectroscopy, and X-ray diffraction are the principal methods used in this work. Studies of the third protein, the membrane ATPase of $H$. saccharovorum, concentrate on the origins of the complex hydrolytic mechanism, the roles played by heavy metals and extraneous ligands that activate or inhibit activity, and its shared features with eubacterial and eukaryotic ATPases.

\section{University of California La Jolla, CA 92093-0116}

\section{Structure, Biosynthesis and Role of Complex Protein-Bound Glycans M.J. Chrispeels, Department of Biology \\ $\$ 96,102$}

The vacuoles of plant cells contain glycoproteins used for storage of nitrogen (storage proteins) and involved in plant defense against insects and mammals (lectins and enzyme inhibitors). We are presently studying the interaction of some of these plant defense proteins with their targets in animals. We are focusing on two classes of proteins, alpha amylase inhibitors (aAl) and lectins, in the common bean, Phaseolus vulgaris, and other leguminous plants. The bean aAl inhibits the amylase of certain insects but not of others. To understand this specificity we recently cloned the amylase of the bruchid Zabrotes subfasciatus and purified the expressed enzyme. We have now started to clone the amylase of a second insect that is not inhibited by the bean inhibitors. Our goal is to co-crystallize enzyme with 
inhibitor. We have found in the common bean two variants of aAl and only one inhibits this amylase. The amino acid sequences of both inhibitors have been obtained. We are now in a good position to study the interaction of the enzyme and the inhibitors. We are investigating a new lectin that interferes with signalling pathways in mammalian cells. We have cloned the lectin from the legume Dolichos lablab, and are now trying to find the related protein in the common bean. We are expressing the Dolichos lablab lectin (DLL) in tobacco seeds to evaluate its biochemical and biological properties.

\section{University of California}

La Jolla, CA 92093-0116

\section{Molecular Structure, Function and Physiology of $\mathbf{K}^{+}$Uptake Channels in Plants J.I. Schroeder, Department of Biology \\ $\$ 91,000$}

Potassium uptake by higher plant cells is of central importance to plant growth, stomatal regulation, cell expansion, nutrition, tropisms, osmoregulation, enzyme homeostasis and plant membrane potential control. Elucidation of the molecular mechanisms by which higher plant cells selectively transport $\mathrm{K}^{+}$is important for manipulation of the above processes. Patch clamp studies on guard cells and many other plant cells have suggested that "inward-rectifying" $\mathrm{K}^{+}$uptake channels provide a molecular pathway for membrane potential control and for low-affinity proton pump-driven $\mathrm{K}^{+}$uptake. These inward-rectifying $\mathrm{K}^{+}$channels interact with metals which are known to show physiological or toxic effects on cation transport, growth and membrane potentials. Arabidopsis $\mathrm{K}^{+}$channel cDNAs have recently been cloned, and we are functionally characterizing the structure and function of two of these cDNAs.

The long-term goal of the proposed research is to attain a quantitative description of physiological $\mathrm{K}^{+}$channel function and of structural domains of $\mathrm{K}^{+}$uptake channel clones responsible for $\mathrm{K}^{+}$channel block and ion selectivity. Furthermore we are determining whether, and to which extent $\mathrm{K}^{+}$uptake channel structure can influence physiological responses and toxic effects of metals on membrane potential and cation transport in specific plant cells. These studies will contribute to a structural dissection of cation transporter functions in higher plants which play important roles in growth, development and environmental responses of plants. 


\section{University of California Los Angeles, CA 90095}

\section{Molecular Biology and Genetics of the Acetate-Utilizing Methanogenic Bacteria R.P. Gunsalus, Department of Microbiology and Molecular Genetics}

$\$ 220,000$ ( 2 years)

Methane formation by the Methanosarcina species is often a rate limiting step during anaerobic biodecomposition in natural and in man made environments. These methanogens are the most versatile with respect to the substrates they use (e.g., acetate, methanol, trimethyl, di-methyl, and methyl-amines, and $\left.\mathrm{H}_{2} / \mathrm{CO}_{2}\right)$. These organisms are also versatile in their ability to adapt to habitats differing in osmolarity (ca. to $1.2 \mathrm{M} \mathrm{NaCl}$ ). We are examining the molecular biology of substrate utilization and osmotic adaptation in these methanogens. We are examining how glycine betaine, $\mathrm{N}$-acetyl- $\beta$-lysine and $\alpha$-glutamate, compounds that are used to osmoregulate the cell in response to increased external salt, are transported into the cell and utilized under varying salt conditions. These adaptive processes appear to be highly regulated by the cell. Genes in $M$. thermophila that are differentially expressed in response to change in osmolarity and to changes in carbon substrate are being identified, cloned, and characterized. We are also using differential CDNA display methods to identify additional genes in the osmoregulation family. Lastly, we are constructing a physical and genetic map of the $M$. thermophila genome that will be of use by a number of investigators in the study of this metabolically talented microbe. The resulting studies should greatly expand our understanding of how methanogens are able to sense environmental change and adapt accordingly for survival and growth.

\section{University of California \\ Los Angeles, CA 90024-1606}

\section{The Gibberellin $A_{20} 3 \beta$-hydroxylase: Isolation of the Enzyme and Its Molecular Biology \\ B.O. Phinney and J. MacMillan, Department of Biology \\ $\$ 75,000$}

The long term objective of our program is to study the biochemical properties of the enzymes that control specific steps in the GA-biosynthetic pathway in maize. The immediate goal of this project is to explore methods for isolating the $G_{20}$-3ß-hydroxylase that catalyzes the metabolism of $\mathrm{GA}_{20}$ to $\mathrm{GA}_{1}$. Our approach is to clone genes controlling steps in the GAbiosynthetic pathway using transposon tagging. We have three transposon-induced dwarf mutants of maize, one from Ac stocks, the other two from Robertson's mutator $(M u)$ stocks. The Ac mutant is allelic to $d 1$ (blocked in the metabolism of $\mathrm{GA}_{20}$ to $\mathrm{GA}_{1}$ ). The two $\mathrm{Mu}$ mutants are blocked early in the GA biosynthetic pathway (bioassay data). We use the appropriate transposon to probe Southern blots of genomic DNA isolated from tall and mutant 
seedlings. We hope to identify a hybridizing band which is unique to the dwarf phenotype. For the $d^{*}-1$ Ac mutant, preliminary studies revealed a $7.3 \mathrm{~kb}$ Sstl genomic DNA fragment which hybridized to our Ac probe and was unique to the dwarf phenotype, however, as we expanded the number of plants examined, we identified dwarfs which lacked the band. We are currentily using the same strategy to examine the two Mu mutants, $d^{\star} E$ and $d^{\star} 9444$.

\section{University of California}

Los Angeles, CA $90024-1606$

\section{Sensory Transduction of the $\mathrm{CO}_{2}$ Response of Guard Cells E. Zeiger, Department of Biology}

Stomatal response to $\mathrm{CO}_{2}$ plays an important role in balancing photosynthesis and water loss in the leaf, and is thus an important factor determining the efficiency of water use by plants. The mechanisms mediating $\mathrm{CO}_{2}$ sensing in guard cells are not well understood. Recent studies in our laboratory have shown that stomata from growth chamber-grown leaves have a very high $\mathrm{CO}_{2}$ sensitivity, as compared with green house-grown leaves. We used reciprocal transfer experiments to determine whether the contrasting $\mathrm{CO}_{2}$ sensitivity of growth chamber and green house-grown stomata ensues from stomatal acclimations to their growth environment. Stomatal sensitivity to $\mathrm{CO}_{2}$ was assessed in isolated stomata from epidermal peels in order to characterize the intrinsic guard cell $\mathrm{CO}_{2}$ response. Stomatal apertures were measured under various $\mathrm{CO}_{2}$ concentrations in darkness. Green house-grown guard cells were insensitive to changes in ambient $\mathrm{CO}_{2}$ whereas growth chamber-grown guard cells had a large response. Guard cells from growth chamber-grown plants transferred to the greenhouse lost their $\mathrm{CO}_{2}$ sensitivity within 2-3 days, whereas guard cells from green house-grown plants transferred to the growth chamber acquired the $\mathrm{CO}_{2}$ sensitivity typical of growth chamber-grown leaves within 5-7 days. This is the first documented acclimation in guard cell sensitivity to $\mathrm{CO}_{2}$. Follow-up studies are characterizing metabolic changes in guard cells associated with changes in $\mathrm{CO}_{2}$ sensitivity, and screening different environmental signals for their effects on the acclimations.

\section{University of California}

Santa Barbara, CA 93106-9610

\section{Regulation and Function of Two Cell Wall Protein Genes in Medicago Roots and Root Nodules J.B. Cooper, Department of Molecular, Cellular, and Developmental Biology $\$ 206,389$ (FY 95 funds/18 months)}

The development of nitrogen-fixing root nodules in legumes involves the controlled infection of host tissues by bacterial symbionts. Previous work showed that expression of several 
nodule-specific PRP genes is induced early in the development of Medicago root nodules and this induction is accompanied by repressed transcript levels for the two major root proline-rich protein genes. The primary objective of this project is to understand the functional significance of these Rhizobium-induced changes in host cell wall architecture. Cloned PRP sequences encoding four members of the Medicago truncatula PRP gene family are being used to examine the role of bacterial invasion signals in the regulation of PRP transcript levels. We found that Rhizobium differentially regulates both PRP transcript levels and PRP deposition in host cell walls, and that bacterial exo-genes, but not nod-genes, are required for regulating transcript levels. A synthetic peptide representing one of the nodule-specific PRP domains was used to generate polyclonal antisera recognizing both root and nodule PRPs on blots, and root hair PRPs in confocal imaging experiments. Immunological approaches using domain-specific antibodies are being developed to examine more precisely the consequences of the Rhizobium-induced alterations in PRP gene expression on host cell wall architecture, and two complementary strategies have been initiated to probe the functional significance of PRPs in nodule development. These studies will begin to elucidate the molecular mechanisms used by symbiotic microbes to regulate host cell wall architecture and successfully invade plant root/nodule tissues.

\section{University of California Santa Cruz, CA 95064}

\section{Regulation of Vacuolar pH in Citrus limon}

L. Taiz, Biology Department

$\$ 98,000$

The vacuolar $\mathrm{pH}$ of the juice sac cells of lemon fruits is considerably lower than that of typical plant tissues ( 2.5 vs. 5.5 ). Our previous studies have implicated the following three factors in the regulation of vacuolar $\mathrm{pH}$ in lemon fruits versus epicotyls: tonoplast proton permeability, intrinsic uncoupling or "slip", and oxidation. One of the unusual features of the fruit V-ATPase in the native membrane is its insensitivity to nitrate. During the past year we have: 1) shown that the $H^{+}$IATP coupling ratio of the fruit V-ATPase is higher than that of the epicotyl V-ATPase, consistent with the absence of slip in the fruit V-ATPase; 2) shown that both fruit and epicotyl V-ATPases have similar "ball and stalk" structures, although the "stalk" may be thicker in the fruit; and 3) completed studies on the purification and reconstitution of the fruit and epicotyl vacuolar $\mathrm{H}^{+}$-ATPases. The subunit compositions of the two purified V-ATPases are nearly identical. Differences include the presence of a $33 \mathrm{kDa}$ subunit in the fruit $\mathrm{V}$-ATPase, as well as some minor subunits between $20-30 \mathrm{kDa}$. Upon reconstitution into artificial proteoliposomes, the fruit $\mathrm{V}$-ATPase became nitrate-sensitive and is indistinguishable from the epicotyl V-ATPase, except for being slightly inhibited by vanadate. Vanadate inhibition decreased with purification, suggesting that it may represent a contaminant ATPase. We are currently testing the role of the native lipids from fruit and epicotyl tonoplasts in cross-reconstitution experiments. 


\title{
Carnegie Institution of Washington Stanford, CA 94305
}

\author{
45. Production of Lipophilic Materials from Plants \\ C.R. Somerville
}

$\$ 448,696$

Our research is focused on understanding the biochemical and genetic mechanisms that regulate the synthesis and accumulation of lipophilic compounds in plants. One aspect of our work is focused on the isolation of genes for transcriptional factors which regulate storage lipid accumulation. The availability of genes for such factors may permit the production of genetically modified plants that accumulate increased amounts of storage oil. A second aspect of our work is focused on elucidating the mechanisms by which plants secrete certain fatty acids and derivatives such as wax. Knowledge of these mechanisms may permit economic production of new kinds of technically useful fatty acids by large-scale fermentation of genetically modified microorganisms. A third aspect of our work is focused on the identification of genes that encode enzymes required for the synthesis of oxygenated fatty acids such as 12-hydroxy oleic acid which are used as feedstocks for the production of a wide variety of chemicals. The availability of these genes is expected to permit the agricultural production of novel high value fatty acids with applications in the synthesis of polymers and other technical products. The development of new plant varieties that accumulate novel oils is expected to provide expanded markets for the agricultural sector while permitting greater reliance on renewable sources of technical materials.

\section{Carnegie Institution of Washington Stanford, CA 94305}

\section{Powdery Mildew Disease Resistance \\ S.C. Somerville, Department of Plant Biology}

$\$ 77,884$

Our long term objective is to characterize genes conferring resistance to powdery mildew diseases, which are caused by obligate fungal pathogens of the Erysiphe genus. Because of the extensive molecular genetic resources available for Arabidopsis, we chose to isolate powdery mildew resistance genes from this plant species. In previous work, five distinct resistance loci were found in five accessions suggesting that powdery mildew resistance in Arabidopsis is as diverse and complex as in crop species like barley. An additional 300 accessions were surveyed for resistance to $E$. cichoracearum isolate UCSC. Sixty-six accessions were resistant to this isolate, providing us with materials for discovering new resistance genes and stronger alleles of known resistance genes.

Two approaches to cloning powdery mildew resistance genes are being pursued. One is the map-based cloning of RPW1 (recognition of powdery mildew). A base population of 100 recombinant inbred F6 (Columbia-gl1 $x$ Kas-1) lines is being tested for disease development 
and for the segregation of linked markers. Another $200 \mathrm{~F} 6$ lines are currently being generated to aid the high resolution mapping of RPW1. The second strategy consists of mapping ESTs with sequence similarity to known resistance genes and comparing their map positions to those of mapped RPW genes. The rationale for the second strategy rests on the observation that most sequenced disease resistance genes share several motifs (e.g., a nucleotide binding site, leucine-rich regions). We believe that at least some powdery mildew resistance genes will exhibit these motifs and may be represented among the R-gene-like ESTs.

\section{University of Chicago Chicago, IL 60637}

\section{Organization and Regulation of the Genes for Nitrogen Fixation in Rhodobacter capsulatus \\ R. Haselkorn, Department of Molecular Genetics and Cell Biology $\$ 100,000$}

Work on individual genes of Rhodobacter capsulatus was brought to a temporary halt in order to gear up for the total sequencing of the genome. Su Zheng's thesis work was published in part, dealing with a novel transport system she found as a byproduct of her search for sigma factor genes. She cloned and sequenced an operon with four genes, encoding an amino acid binding protein, two transmembrane transporters, and an ATP-binding cassette. Deletions and direct transport measurements showed that this operon was responsible for the transport of aspartate, asparagine, glutamate and glutamine. The gene encoding the ABC protein and another encoding one of the transmembrane proteins were used as probes of the high resolution hybridization template, which contains 560 restriction fragments from the chromosome displayed according to their position on the physical map. The results showed at least 15 related operons distributed throughout the chromosome.

The sequencing project has progressed nicely. At present, we have $500 \mathrm{~kb}$ finished and annotated, another $500 \mathrm{~kb}$ finished but not completely annotated. The sequencing is being done partly in Prague in collaboration with the Czech Academy. They have completed 150 $\mathrm{kb}$, including the complete vitamin B12 biosynthetic gene cluster and some other potentially useful genes. In addition to sequencing, we have extended our previous use of the GTA to knock out genes by deletion. The general method developed has been proved to work for three more cosmids, raising the amount of DNA that can be deleted without phenotype on complete medium to $150 \mathrm{~kb}$. Instructive attempts were made to use the GTA to delete essential genes. In one such case, we documented the recovery of a transconjugant in which the GTA recombination event began at one end of the targeted segment, proceeded to the essential gene, and then skipped the rest of the target, leaving the essential gene and everything downstream of it in the chromosome. We expect to complete another Mb of sequence during the coming year, at a cost per finished base of about one-fifth the amount charged by others for genome sequencing. 


\section{University of Chicago Chicago, IL 60637}

\section{Role of HSP100 Proteins in Plant Stress Tolerance S. Lindquist, Department of Molecular Genetics and Cell Biology $\$ 198,060$ (FY 95 funds/18 months)}

The inability of plants to withstand high temperatures and water stress provides the single greatest limit to agricultural productivity. We are investigating mechanisms of improving stress tolerance. All organisms acquire some ability to survive higher temperatures and other stresses when they are given a conditioning preheat treatment. During conditioning treatments, heat-shock proteins or HSPs are expressed. The first HSP demonstrated to play an important role in high temperature survival is the HSP100 protein of yeast, an organism that offers many advantages for rapid genetic analysis. We have found that although yeast cells carrying mutations in HSP104 die at high temperature, they can be rescued by the homologous HSP100 protein of the plant, Arabidopsis thaliana. This demonstrates that the functions of HSP100 have been conserved in thermotolerance for over 1 billion years. Since the HSP100 protein is not toxic when over-expressed, we hope to improve the thermotolerance of plants by increasing the production of HSP100. This year we have generated vectors for the constitutive and heat-inducible expression of yeast and plant HSP100 proteins in Arabidopsis as well as vectors for the production of antisense genes to inhibit protein expression. Transgenic plants have been created for each construct and we are now examining them for changes in HSP100 protein expression and thermotolerance. We are also comparing the biochemical properties of the purified yeast and plant proteins in terms of peptide stimulated ATPase activity and oligomerization.

\section{University of Chicago Chicago, IL 60637}

49. Signal Transduction in Plant Development: Chemical and Biochemical
Approaches to Receptor Identification
D.G. Lynn, Searle Chemistry Laboratory

Striga asiatica is a small chlorophyll-containing angiosperm that has developed the remarkable ability to establish a vascular connection with another plant. The development of the attachment organ, the haustorium, is one of the most rapid organogenesis events known. Since this parasitic ability is wide-spread among the plant families, it has generally been assumed that the development of the attachment organ relies on ubiquitous plant developmental programs. It was therefore quite surprising when it was discovered that relatively simple host-derived phenolic compounds were sufficient to induce haustorial development. The subsequent discovery that simple phenolic compounds had evolved as the primary signal initiating vir expression in Agrobacterium and nod expression in Rhizobium and 
that some of these same compounds were important in plant growth and development have raised fundamentally new questions about the role of these compounds in plants.

Subsequent work on the phenolic signals in Striga has provided evidence that the compounds are detected via a chemical reaction, again quite distinct from our current models of hormone/growth factor detection by membrane localized binding proteins. Evidence is presented that the recognition mechanism is a redox reaction most likely controlled by plasma membrane localized oxidoreductases. While the existence of these redox systems have been demonstrated in both plants and animals, only recently has convincing evidence connecting $e^{-}$transport with plant development emerged.

Striga therefore can teach us not only about the development of host-parasite interactions but also represents a rapid and well defined developmental transition where the chemistry of the inducing signal can be exploited to provide insights into the developmental process. Work over the past year has identified several genes which are controlled by these signals and are of functional importance to the developmental transition. We propose to use this chemistry both to provide information about the initial detectors and to define the signal transduction events in controlling haustorial development. These events will be compared and contrasted with other plant developmental transitions.

\section{University of Chicago}

Chicago, IL 60637

\section{Molecular Genetic Analysis of Biophotolytic Hydrogen Production in Green Algae \\ L. Mets, Department of Molecular Genetics and Cell Biology \\ $\$ 100,000$}

Several species of green algae have the unique biochemical capability of producing gaseous hydrogen from water using solar energy harvested by the photosynthetic apparatus, a process called biophotolysis. An understanding of factors that promote as well as limit biophotolysis may lead to a highly efficient solar energy conversion system that directly yields hydrogen gas as the energy storage medium. We are exploiting the powerful array of genetic analysis and molecular genetic manipulation techniques that have been developed for the green alga Chlamydomonas reinhardtii to investigate the molecular mechanisms underlying biophotolysis.

Efficient hydrogen production occurs only under anaerobic or anoxic conditions. This feature limits the utility of biophotolysis, since oxygen is a necessary co-product with hydrogen. Hydrogenase, the key enzyme that couples energy harvested by the photosynthetic light reactions in the chloroplast to hydrogen evolution is not synthesized except in the absence of oxygen. The mechanism of this regulatory effect of oxygen is being investigated as part of this project. The activity of the hydrogenase in green algae is also directly destroyed by molecular oxygen, although the detail is unknown. The enzyme from Chlamydomonas has 
only one subunit, making it an ideal target for exhaustive mutagenesis to test the possibility that an oxygen-insensitive form can be developed. We have initiated an effort to generate genetically altered enzymes with different degrees of oxygen sensitivity. Analysis of these mutant enzymes will help to build a picture of the mechanism of oxygen inactivation and support directed efforts to develop fully oxygen-insensitive activity.

\section{University of Chicago Chicago, IL 60637}

\section{Analysis of Cell-Cell Interactions during Arabidopsis Reproduction D. Preuss, Department of Molecular Genetics and Cell Biology $\quad \$ 109,999$}

Plants have a remarkable ability to interact favorably with pollen of the appropriate species, while inhibiting foreign pollen. To clarify the molecular basis for this discrimination, we have identified genes that are critical for pollen communication with female pistil tissues. We have shown that mutations in these genes can affect the abundance of proteins on the surface of pollen and pistil cells. Our work focuses on understanding these surface molecules, and these studies will likely provide valuable tools for manipulating plant resources, both in agriculture and in natural ecosystems.

We are exploring the initial interactions between pollen and the receptive stigma cells on the pistil surface by determining the function of two pollen surface proteins. In particular, we plan to identify mutations in these proteins and then to test whether the surface proteins play a critical role in binding to stigma cells, in mediating communication with the stigma, or in specifying pollen identity. Subsequent interactions between pollen tubes and ovules will be investigated using a newly identified mutation that alters pollen tube guidance. Because this mutation alters the ability of pollen tubes to adhere to ovule cells, we plan to explore the requirements for the adhesive interactions by characterizing the mutant defects and mapping the altered genes. These studies will expand.our knowledge of the unique signaling mechanisms that enable plant cells to communicate, despite their thick extracellular walls.

\section{Clemson University \\ Clemson, SC 29634-1903}

\section{The Magnesium Chelation Step in Chlorophyll Biosynthesis}

J.D. Weinstein, Department of Biological Sciences

$$
\$ 184,010 \text { (FY } 95 \text { funds/2 years) }
$$

In photosynthetic organisms, the synthesis of new energy generating membranes requires the coordinate synthesis of proteins, their associated cofactors, and various lipids. The important cofactors, chlorophyll and heme, share a common biosynthetic pathway diverging at the point 
of metal ion insertion. Mg-chelatase catalyzes the insertion of magnesium into protoporphyrin-IX, the first step unique to chlorophyll synthesis. Our work is focused on the mechanism and regulation of this enzyme as it is a key to understanding how plants produce the proper proportion of chlorophyll and heme from a common pathway. By subfractionation (affinity chromatography and molecular size ultrafiltration) of pea chloroplast extracts, we have demonstrated that this enzyme consists of throu separate protein components which are required for activity. Two of these components are required for an ATP-dependent activation; this step is followed by metal ion insertion, which also requires the third component. The $\mathrm{Mg}$-chelatase of photosynthetic bacteria and cyanobacteria is encoded in three genes. In higher plants two genes (Olive and $\mathrm{CH}-42$ ) have been identified which are homologous to two of the bacterial genes (Bch $\mathrm{H}$ and Bch I, respectively). We have now cloned the putative third gene from higher plants. The protein expressed from that gene will be tested for activity in reconstitution assays with the subfractions isolated from chloroplasts extracts. The previously identified higher plant genes from peas will also be cloned and expressed with the goal of having all three components from peas expressed in an active form.

\section{Cold Spring Harbor Laboratory Cold Spring Harbor, NY 11724}

\section{The Suppression of Mutations Generated by Mu Transposons in Maize R.A. Martienssen and V. Sundaresan

Mutations caused by the insertion of Mutator $(M u)$ transposable elements are frequently dependent on the presence of active autonomous elements elsewhere in the genome. Such mutations are phenotypically suppressed when $M u$ activity is lost, resulting in wild-type plants. Recessive alleles of this class at four different loci (hcf106, a1, Kn1, vp1) have Mu1 or Mu8 elements in the promoter region of the gene. In the case of hcf106, phenotypic suppression is accompanied by transcript initiation within the Mu1 element that results in the production of a functional chimeric Mu1::hcf106 message. This results in a pseudorevertant phenotype that can be used to monitor loss of $M u$ activity both germinally and in somatic sectors. Analysis of double mutant combinations of Les28, hcf106 and Kn1-mum2 have been used to demonstrate that co-ordinate loss of activity occurs in sectors during development. This property of suppressible alleles can be used for mosaic analysis as loss of activity results in adjacent sectors of mutant and wild-type tissue.

In order to isolate new alleles of hcf106 that respond differently to $M u$ activity, we have developed a simple technique for site-selected transposon mutagenesis in maize. Rare individual plants carrying derivative alleles were selected by PCR amplification of specific fragments from pools of seedlings. By cross-referencing two-dimensional pools, individual plants can be rapidly identified with a single round of PCR. Three new alleles (one deletion and two insertions) were recovered among 800 gametes screened. Unlike the progenitor allele, two of these alleles no longer require $M u$ activity for their phenotypic effects. This 
technique could be applied to maize genes of known sequence allowing insertions and deletions to be recovered in vivo. In many cases, these mutations would allow the function of the gene to be determined from the mutant phenotype. This technique has potential application in genome analysis where most genes are defined by sequence information alone.

\section{University of Connecticut}

Storrs, CT 06269-3125

\section{Analysis of Cell Wall Properties in Polysaccharide Mutants of Arabidopsis W.-D. Reiter, Department of Molecular and Cell Biology}

$$
\$ 133,700 \text { (FY } 95 \text { funds/18 months) }
$$

The mechanical properties of plant cell walls and the synthesis of new wall material play pivotal roles for the determination of cell shapes and sizes. Accordingly, an understanding of plant growth and development requires an understanding of the mechanisms underlying the synthesis of new cell wall material, and the assembly of individual cell wall components into a matrix with both growth-regulating and structural roles. In an effort to address cell wall-related issues by a genetic approach, we have been characterizing mutants of the plant model system Arabidopsis showing changes in the sugar composition of cell wall material. Two of the cell wall mutants (mur1 and mur4) are partly deficient in the synthesis of the monosaccharides L-fucose and L-arabinose, respectively, which are important building blocks for cell wall polysaccharides. Biochemical data on both mutant lines indicate some redundancy in the biosynthetic pathways leading to these cell wall precursors. We are currently studying the MUR1 gene and a related gene encoding isoforms of GDP-D-mannose-4,6-dehydratase, the enzyme catalyzing the first step in the formation of GDP-L-fucose from GDP-D-mannose. Another gene believed to act downstream of the MUR1 gene is also being analyzed on the molecular level to establish its function and expression pattern. We plan to combine tools of conventional and reverse genetics to accomplish a stepwise elimination of the genetic redundancy in precursor synthesis to determine the effects of polysaccharide modifications on plant anatomy, cell wall ultrastructure, and the biophysical properties of the wall.

\section{University of Connecticut \\ Storrs, CT 06269}

\section{Sugar Transport and Metabolism in Thermotoga \\ A.H. Romano and K.M. Noll, Department of Molecular and Cell Biology $\$ 87,000$ (FY 95 funds/17.5 months)}

Thermotoga neapolitana is a strictly anaerobic fermentative hyperthermophilic bacterium that represents a slowly-evolving bacterial lineage that gave rise to the first known heterotrophic 
bacteria. We have studied transport systems for D-glucose and ss-D-galactosides in this organism using the non-metabolizable sugar analogs 2-deoxy-D-glucose and methyl-ss-D-thiogalactopyranoside, respectively. We found that both these sugars are taken up via active transport systems that appear to be dependent on $\mathrm{Na}^{+}$, and energized by ion gradients generated by ATP derived from substrate-level phosphorylation. There was no evidence for the presence of a PEP:sugar phosphotransferase system. The mechanism of the $\mathrm{Na}^{+}$requirement is being investigated to gain further insight into how these hyperthermophiles generate and maintain ion gradients under presumed conditions of increased membrane permeability to ions at high temperatures.

While the glucose transport system of $T$. neapolitana appears to be constitutive, systems for both ss-galactoside transport and catabolism (ss-galactosidase) are inducible, and subject to CAMP-independent repression by glucose (catabolite repression). However, glucose did not inhibit ss-galactoside uptake by induced cells, nor did glucose provoke efflux of pre-accumulated intracellular ss-galactoside. Thus, the phenomena of inducer exclusion and inducer expulsion, mechanisms which putatively involve specific protein components of the PEP:sugar phosphotransferase system in certain modern bacteria, appear not to be present in $T$. neapolitana. Further study of the regulation of ss-galactoside transport and catabolism in this organism at the genetic, biochemical, and physiological levels should provide insight into the evolution of catabolic control systems in bacteria.

\section{Cornell University}

Ithaca, NY 14853-2703

\section{Cytoplasmic Male Sterility and Mitochondrial Function During Microsporogenesis \\ M.R. Hanson, Division of Biological Sciences \\ $\$ 100,000$}

Male reproductive development is often disrupted before or during meiosis in cytoplasmic male sterility (CMS) plants. Electron microscopic studies have shown alterations in mitochondrial appearance at this time in development. Mitochondrial genes are differentially expressed in male reproductive tissue. We are using both two-photon confocal microscopy and two-photon fluorescence microscopy, in conjunction with membrane-potential sensitive dyes and the green fluorescent protein, to analyze mitochondrial movement, numbers, and respiratory activity in vivo in isonuclear wild-type, CMS, and CMS-fertility restored Petunia lines. Transgenic plants containing the green fluorescent protein targeted to mitochondria and other organelles are being produced so that subcellular structures can be visualized in reproductive tissue. Portions of the CMS-associated gene will be expressed to examine the role of this mitochondrial gene in the aberrant pollen development. 


\section{Cornell University \\ Ithaca, NY 14853-5908}

\section{Signal Transduction in the Pollen-Stigma Interactions of Brassica J.B. Nasrallah and M.E. Nasrallah, Section of Plant Biology, Division of Biological Sciences

Self-pollination in Brassica is prevented by the action of genes that reside at the highly polymorphic self-incompatibility $(S)$ locus. Self/nonself recognition is attributed to the activity of identical $S$ haplotypes in pollen and stigma, and results in the inhibition of self pollen at the surface of stigmatic papillar cells. We are using the Brassica self-incompatibility system as an experimental model for investigating pollination responses and mechanisms of cell-cell interaction in higher plants. To date, our work has focused on the characterization of the $S$ locus Receptor Protein Kinase (SRK), one of two S-locus encoded proteins that have been identified in our laboratory. SRK is a receptor-like protein that exhibits serine/threonine kinase activity and shares a high degree of sequence similarity with the secreted S-Locus Glycoprotein (SLG) that is also encoded by the S locus. SRK and SLG are both required for the operation of self-incompatibility and are coordinately regulated, being expressed athighest levels in the papillar cells of the stigma surface. By biochemical and immunochemical analysis of stigma proteins and of proteins extracted from transgenic tobacco tissue that expresses SRK, we have demonstrated that the SRK protein is a plasma membrane localized protein. Our continued biochemical and molecular studies of the system are aimed at determining if and how the cell surface receptor is activated by contact with self pollen and at identifying intermediates in its signaling pathway. It is anticipated that the deciphering of SRK-mediated signaling will contribute to the elucidation of the general principles that underlie receptor-mediated signaling and cell-cell communication in higher plants.

\section{Cornell University Ithaca, NY 14853-8101}

\section{Regulation of Denitrification in Rhodobacter sphaeroides J.P. Shapleigh, Section of Microbiology $\$ 105,000$ (FY 95/18 months)}

Bacteria have the capacity to respire compounds other than oxygen. Nitrate is one of the many compounds that can serve as an alternative respiratory substrate. Nitrate respiration to nitrogen gas is referred to as denitrification. Our laboratory is interested in understanding how bacteria regulate the synthesis of proteins required for denitrification. In particular, we want to understand the coordination of gene expression and to identify critical regulatory proteins. The bacterium we will use for our studies is a denitrifying variant of Rhodobacter sphaeroides. This is an ideal organism to use because genetic systems are well developed and a great deal is known about other components of the respiratory chain. 
We have focused our effort on understanding the regulation of the genes encoding nitrite and nitric oxide reductase, which together carry out nitric oxide synthesis and reduction. Nitric oxide is an obligatory intermediate of denitrification and is also an important regulatory molecule in higher organisms, including humans. Using various mutants we have shown that nitric oxide production is required for expression of nitrite and nitric oxide reductase. A gene encoding a protein that may be the direct regulator of the two reductases has been characterized. Several other genes that encode products required for reductase expression have also been isolated and characterized. Our studies demonstrate that bacteria, like humans, can utilize nitric oxide as an important regulatory molecule. We are currently working to understand the regulatory mechanism used by Rhodobacter to detect nitric oxide and activate gene expression.

\section{Cornell University}

Ithaca, NY 14853

\section{Effects of Freezing and Cold Acclimation on the Plasma Membrane of Isolated Cereal Protoplasts \\ P.L. Steponkus, Department of Soil, Crop and Atmospheric Sciences $\$ 108,000$}

The ultimate goal of this project is to provide a mechanistic understanding of the cellular and molecular aspects of freezing injury and cold acclimation to provide for rational strategies for the improvement of freezing tolerance of crop species. The current objectives are to determine the mechanism(s) by which cytosolic sugars increase the cryostability of cellular membranes and alter the lyotropic phase behavior of phospholipids. When dried in the presence of a sugar (e.g., glucose, sucrose or trehalose), the dehydration-induced increase in the gel-to-liquid crystalline $\left(L_{\beta}-L_{\alpha}\right)$ phase transition temperature $\left(T_{m}\right)$ is greatly minimized such that the $T_{m}$ is increased only a few degrees above the $T_{m}$ of the fully hydrated lipid. This is a consequence of the osmotic effect of sugars and the increased viscosity of the sugar matrix rather than specific sugar-lipid interactions as is often inferred. At lower hydrations, when the sugar matrix undergoes a glass transformation during cooling while the phospholipid is in the $L_{\alpha}$ phase, the $T_{m}$ is depressed below the $T_{m}$ of the fully hydrated lipid. The magnitude of the depression in the $T_{m}$ varies (from 12 to $50^{\circ} \mathrm{C}$ ) with the lipid species but is independent of the sugar species provided the sugar undergoes a glass transformation at a temperature at which the phospholipid is in the $L_{\alpha}$ phase. At equal hydrations, the $T_{g}$ of trehalose>sucrose>glucose. Thus, for a particular lipid species at a particular hydration, depression of the $T_{m}$ may occur in sugar-lipid mixtures containing trehalose but not in those containing sucrose or glucose. 


\section{Cornell University}

Ithaca, NY 14853

60. Genetic Control of Nitrate Assimilation in Klebsiella pneumoniae V.J. Stewart, Section of Microbiology, Division of Biological Sciences $\$ 94,000$

Klebsiella oxytoca (pneumoniae) is an enteric bacterium closely related to Escherichia coli. Nitrate and nitrite are important nitrogen sources not only for $K$. oxytoca, but also for many other microorganisms and most plants. In the absence of ammonium, nitrate (or nitrite) induces the synthesis of assimilatory nitrate reductase and assimilatory nitrite reductase, which act in sequence to convert nitrate to ammonium. One aspect of our work has been to identify and characterize the structural genes involved in nitrate assimilation, which are organized in the nasFEDCBA operon. The nasFED genes encode a nitrate uptake system, whereas the nasCBA genes encode assimilatory nitrate and nitrite reductases. A positive regulatory gene, nas $R$, is located immediately upstream of the nas $F$ operon. We have recently constructed several in-frame nonpolar deletions throughout the nasFoperon, and we are beginning to use these strains in explorations of nitrate and nitrite uptake. Other current studies are designed to explore the regulation of nasF operon expression. Transcription is induced by nitrate or nitrite during nitrogen-limited growth. The global NtrC protein mediates general nitrogen control of nasF operon transcription. Nitrate and nitrite induction is mediated by transcriptional antitermination. A factor-independent transcriptional terminator is located within the transcribed leader region upstream of the nasF gene. In the presence of nitrate or nitrite, the NasR protein inhibits termination at this site, thereby allowing high-level nas $F$ operon expression. We determined the nucleotide sequence of the nas $F$ operon leader region of $K$. pneumoniae (aerogenes), in order to make phylogenetic comparisons with the $K$. oxytoca sequence. Sequence alignment analysis indicates that two distinct RNA secondary structures may be important for regulated antitermination.

\section{Cornell University Ithaca, NY 14853}

\section{Studies of the Genetic Regulation of the Thermomonospora fusca Cellulase Complex \\ D.B. Wilson, Section of Biochemistry \\ $\$ 94,000$}

The long term goal of our research is to produce cellulases with higher activity in degrading biomass cellulose to sugar that will allow the economical production of ethanol from biomass. The current goals are: one, to clone the gene for and characterize the activity of a Thermomonospora fusca regulatory protein that functions in the induction of six $T$. fusca cellulases and two, to determine the molecular mechanism of $T$. fusca endoglucanase E2. From our previous work it seems likely that the regulating protein will be modified in response to the inducer to produce the active form, possibly by phosphorylation. We will try to get 
evidence for this by preparing antibodies against the regulatory protein and using them to identify the inactive form of the protein in uninduced cells. We have constructed a number of mutants in the E2 catalytic domain by site directed mutagenesis and are characterizing the mutant proteins by determining their activity on four different cellulosic substrates and by measuring their affinity for a number of oligosaccharides. The properties of several mutants provide strong evidence that a conformational change occurs after substrate binding that appears to move a loop over the active site cleft holding in the substrate. This change is required for high activity. We plan to see if we can obtain more evidence for this change using either X-ray crystallography or protein NMR. We also have obtained two different mutants that increase the activity of E2 on both CMC and soluble oligosaccharides. We are combining the mutations to see if their effects are additive. The properties of a mutant in a proposed catalytic base are surprising since the mutant enzyme binds oligosaccharides with $1 / 20,000$ the affinity of wild type E2. This suggests that this residue may be required for binding rather than functioning directly in catalysis. We will continue to mutate residues in E2 that appear from the structure to be involved in either substrate binding or catalysis and to characterize the mutant proteins.

\section{Cornell University Ithaca, NY 14853-8101}

\section{Conversion of Acetic Acid to Methane by Thermophiles S.H. Zinder, Department of Microbiology}

The objective of this project is to provide an understanding of thermophilic anaerobic microorganisms capable of breaking down acetic acid, the precursor of two-thirds of the methane produced by anaerobic bioreactors. Recent results include: 1) the demonstration that thermophilic cultures of Methanosarcina and Methanothrix show minimum thresholds for acetate utilization of 1-2.5 $\mathrm{mM}$ and 10-20 $\mu \mathrm{M}$ respectively, in agreement with ecological data indicating that Methanothrix is favored by low acetate concentration; 2) the demonstration that, in contrast to Methanosarcina, Methanothrix strain CALS-1 did not accumulate $\mathrm{H}_{2}$ during methanogenesis from acetate, but instead accumulated $\mathrm{CO} ; 3$ ) the purification and characterization of a carbon monoxide dehydrogenase/corrinoid protein complexfrom cell-free extracts of Methanothrix strain CALS-1 which shows different subunit sizes from that of Methanosarcina; 4) the demonstration of methanogenesis from acetate and ATP in cell-free extracts of strain CALS-1. Methanogenesis occurred at a high rate $\left(100-300 \mathrm{nmol} \mathrm{min}{ }^{-1}\left[\mathrm{mg}^{2}\right.\right.$ protein $]^{-1}$ ), and much lower rates were obtained with acetyl-CoA or acetyl-phosphate as substrates; 5) the demonstration that methanogenesis from acetate in Methanothrix was independent of $\mathrm{H}_{2}$ and other electron donors, in contrast to Methanosarcina, and in agreement with results obtained in whole cells; 6 ) the demonstration that coenzyme $A$, coenzyme $M$, tetrahydrosarcinapterin, and HS-HTP are required for methanogenesis from acetate by cell-free extracts. Current research is centered on biochemical factors which allow thermophilic Methanothrix to compete with Methanosarcina. 


\section{University of Delaware}

Lewes, DE 19958

\section{Metabolic Mechanisms of Plant Growth at Low Water Potentials J.S. Boyer, College of Marine Studies}

In land plants, growth is more frequently limited by water than by any other environmental resource. The project objective is to identify the underlying molecular mechanisms causing growth limitations by focusing on the process of cell enlargement. Studies so far have shown that, in localized growing regions of germinating soybean seedlings, turgor in most of the cells was completely maintained when water potentials were low enough to inhibit growth. However, gradients in water potential decreased between the vascular tissue and the enlarging cells, and growth was inhibited. A few $h$ later, the extensibility of the cell walls decreased and an extractable $28 \mathrm{kD}$ protein accumulated in the wall fraction. To determine whether the protein was present in the cell walls of the intact plants, roots were pressurized sufficiently to force exudate onto the surface of the stems. The exudate contained the protein, which had acid phosphatase activity. There was acid phosphatase activity in the cytoplasm as well. Measurements of phosphorylated intermediates and of starch and cell wall products showed inhibited deposition of the products but not as much as the growth inhibition so that the products slowly accumulated. There was no change in phosphorylated intermediates. Instead, substrates including inorganic phosphate accumulated. Experiments are underway to separate these metabolic effects from gradient effects using root pressurization.

\section{Duke University \\ Durham, NC 27708}

\section{Molecular, Genetic and Physiological Analysis of Photoinhibition and Photosynthetic Performance \\ J.E. Boynton, N.W. Gillham and C.B. Osmond, DCMB Group, Departments of Botany and Zoology \\ $\$ 112,000$}

This project combines molecular genetics, biochemistry and physiology to understand the relationship between photosynthetic performance in vivo and the structure of the multifunctional D1 reaction center protein of Photosystem II. Plants and algae exposed to full sunlight frequently suffer photoinhibitory damage to their chloroplast encoded D1 protein, which reduces photosynthetic efficiency. The green alga Chlamydomonas is well suited for these studies because of its excellent genetics and ease of chloroplast transformation. We have isolated and characterized D1 mutants with 12 of the possible 20 amino acids inserted at the $\mathrm{Ala}_{251}$ residue - five nonphotosynthetic (J. Biol. Chem. in press) and seven with variously reduced photosynthetic capacity (manuscript in preparation). We have also isolated 46 mutants which partially suppress the high light sensitivity of the $\|_{251}$ and $\operatorname{LeU}_{251} D 1$ 
mutants or reduce the sensitivity of wild type to sun light irradiance. Certain suppressors may alter D1 synthesis and turnover or the zeaxanthin/violaxanthin protection pathway. Our characterization of initial photoinhibition and subsequent photoacclimation events when .wildtype Chlamydomonas grown in low light is transferred to high light will appear in Plant Mol. Biol. We are now determining whether transient downregulation Rubisco LSU synthesis and stable upregulation of D1 synthesis following the light shift results from binding of specific transacting proteins to their untranslated mRNA leaders. Identification of gene products involved in mediating photosynthetic performance under photoinhibitory conditions that effect D1 structure/function directly or modulate its synthesis and photoprotection may permit future manipulations of land plants to utilize light more efficiently.

\section{Florida State University}

\section{Tallahassee, FL 32306}

\section{Role of Sucrose in Modulating Stomatal Aperture W.H. Outlaw, Jr., Department of Biological Science}

Gas exchange between a leaf and the atmosphere occurs through adjustable stomata, each of which is defined by the surrounding pair of guard cells. Certain conditions stimulate guard cells to accumulate potassium salts. The special cell-wall architecture forces the cells to bow outward upon the consequent osmotic-water influx; this deformation enlarges the pore. Stomal closure occurs when the guard-cell pair loses solutes. The aperture size is generally a compromise between the opposing priorities of permitting $\mathrm{CO}_{2}$ uptake and avoiding $\mathrm{H}_{2} \mathrm{O}$-vapor loss. As $\mathrm{CO}_{2}$ is required for photosynthesis and water is usually the most limiting resource for a terrestrial plant, regulation of stomatal-aperture size is one of the most crucial aspects of a plant's physiology.

As implied, the prevailing paradigm is that fluctuations in the concentrations of potassium salts in guard cells power stomatal movements. Recently, we (e.g., FEBS Lett 362: 180-184) and others (e.g., Plant Physiol 111: 1051-1057) reported that sucrose has an osmotic role potentially equal to that of potassium salts. Under the conditions we have studied, sucrose accumulation in the guard-cell cytoplast is secondary, and we have focused on the accumulation of sucrose in the guard-cell apoplast, which we study by use of quantitative histochemical methods. Our present experiments test the hypothesis that sucrose accumulation there results from photosynthesis in the mesophyll. If our hypothesis is correct, we will postulate that sucrose accumulation in the guard-cell apoplast, and consequent diminution of stomatal-aperture size, is one means by which plants measure the rate of transpiration. 


\section{University of Florida Gainesville, FL 32611}

\section{Ethanologenic Enzymes of Zymomonas mobilis L.O. Ingram, Department of Microbiology and Cell Science}

Zymomonas mobilis is widely recognized as an organism with commercial potential for fuel ethanol production. Although the range of sugars fermented by this organism is limited, ethanol production rates by $Z$. mobilis are 5 -fold to 15 -fold faster than traditional yeasts. Our studies have focussed on understanding the basis for this exceptionally fast rate of fermentation. Rapid fermentation is achieved in part by producing very high levels of the enzymes which convert sugars into ethanol. These enzymes alone represent approximately half of the soluble cell protein. The mRNAs encoding these enzymes are remarkably stable, allowing repeated translation. The respective genes are expressed from multiple promoters. Ribosomal-binding sites and codon usage appear optimized. The glycolytic enzyme products are quite stable with little turnover by proteolysis. The control of sugar flow through metabolism to ethanol rests primarily with glucose-6-phosphate dehydrogenase when cells are their peak activity. Genetically increasing the level of this enzyme further increases the rate of fermentation. However, after a $15 \%$ increase in flux, other glycolytic enzymes become limiting and dominate flux control. The exceptional rate of glycolysis by $Z$. mobilis may also result from the low yield of ATP (net 1 per glucose) and the development of specific energy spilling reactions. These energy spilling reactions regenerate ADP, allowing glycolysis to continue. Genes encoding all three major ATPases activities in $Z$. mobilis have now been cloned. Knockout mutations are being investigated to establish function.

\section{University of Florida Gainesville, FL 32611}

\section{Gene-Enzyme Relationships of Aromatic Amino Acid Biosynthesis in Higher Plants \\ R.A. Jensen, Department of Microbiology and Cell Science

The biosynthesis of aromatic amino acids in higher plants is of great significance, not only because of the role of these amino acids in protein synthesis, but because they are precursors of a vast array of compounds of biotechnological and medical interest. Some of the pathway enzymes also offer potential targets for design of new herbicides. Full-length cDNAs encoding five plastid-localized enzymes will be obtained from both Nicotiana and Arabidopsis. These correspond to four proteins: the bifunctional AroD $E$ (having catalytic domains for dehydroquinase and shikimate dehydrogenase) and the postprephenate enzymes: prephenate aminotransferase, arogenate dehydrogenase, and arogenate dehydrogenase. $\mathrm{N}$-terminal amino acid sequencing will identify the putative transit peptides. The genomic DNAs will be isolated and sequenced. Intron-exon organization will be 
determined. Molecular studies will include determination of subcellular location using monospecific antibody and immunogold electron microscopy, analysis of the organ-specific expression pattern of transcript abundance, determination of cell-specific expression in transgenic plants using GUS fusions, effect of antisense RNA and sense RNA manipulations, and demonstration of the import of unprocessed protein precursors into intact chloroplasts. Approaches that might lead to the possible identification of cDNAs specifying cytosolic enzymes of aromatic amino acid biosynthesis are developed.

\section{University of Georgia Athens, GA 30602-7229}

68. The Metabolism of Hydrogen by Extremely Thermophilic Bacteria M.W.W. Adams, Department of Biochemistry and Molecular Biology, and Center for Metalloenzyme Studies $\$ 202,000$ (FY 95 funds/2 years)

Extremely thermophiles or "hyperthermophiles" are a unique group of microorganisms that have the remarkable property of growing optimally near and above $100^{\circ} \mathrm{C}$. They have been isolated mainly from marine volcanic environments, including deep sea vents. We grow some of these organisms in large scale culture (600 liters) and are studying their pathways of hydrogen $\left(\mathrm{H}_{2}\right)$ metabolism. The archaea (formerly archaebacteria), Pyrococcus furiosus (Pf, $\left.T_{\max } 105^{\circ} \mathrm{C}\right)$. Thermococcus litoralis $\left(T_{\max } 98^{\circ} \mathrm{C}\right)$ and "ES $-4^{\prime \prime}\left(T_{\max } 110^{\circ} \mathrm{C}\right)$ produce $\mathrm{H}_{2}$ by the fermentation of carbohydrates and/or peptides and their growth is stimulated by tungsten (W), an element seldom used in biology. From these organisms we have purified NADP-dependent, nickel-containing hydrogenases, redox proteins such as ferredoxin and rubredoxin, a ferredoxin NADP oxidoreductase (FNOR), four different types (POR, KGOR, IOR and POR) of 2-ketoacid ferredoxin oxidoreductases (KAORs), and three different types of tungsten-containing aldehyde ferredoxin oxidoreductase. One of the tungstoenzymes (GAPOR) couples glyceraldehyde-3-phosphate oxidation to $\mathrm{H}_{2}$ production in a novel glycolytic pathway, while the other two (AOR and FOR) and the KAORs are part of an unusual pathway for peptide catabolism. Specifically, the KAORs function as 2-keto acid decarboxylases and the various aldehydes that they generate are oxidized to the corresponding acid by AOR and FOR. The reductant that is produced is converted to $\mathrm{H}_{2}$ via FNOR, NADP and hydrogenase. The genes for ferredoxin, rubredoxin, FOR, AOR, POR and VOR from Pf have been cloned and sequenced and, in collaborative studies with D. Rees (Caltech), the crystal structure of Pf AOR has been determined. This was the first structure for a hyperthermophilic, a tungsten-containing, or a pterin-containing enzyme. Since molecular $\mathrm{H}_{2}$ plays a central role in the commercial production of many chemicals, a long term objective of this research is to assess the utility of hyperthermophilic hydrogenases and various oxidoreductases in industrial energy conversions. 


\section{University of Georgia}

Athens, GA 30602-4712

\section{CarbBank - A Structural and Bibliographic Database for Complex Carbohydrates}

P. Albersheim and S. Doubet, Complex Carbohydrate Research Center

$\$ 125,000^{*}$

The Windows-compatible, computerized database (Complex Carbohydrate Structure Database, CCSD) and database management system (CarbBank) were created by scientists at the Complex Carbohydrate Research Center (CCRC) in collaboration with scientists in Europe. Building the database and making the software available to researchers worldwide were the initial goals of the project. Towards this goal, distribution and software development licenses have been formed with several major biosequence database providers: the National Center for Biotechnology Information (NCBI) of the National Library of Medicine at the U.S. National Institutes of Health $(\mathrm{NIH})$, the Protein Information Resource (PIR) International [composed of the National Biomedical Research Foundation (NBRF) in the U.S., the Martinsreid Institute for Protein Sequences (MIPS) in Europe, and the Japan International Protein Information Database (JIPID) in Asia]. NCBI and MIPS provide the CCSD/CarbBank on their Internet servers, while MIPS, NBRF, and JIPID distribute CCSD/CarbBank on their Atlas CD-ROM (updated quarterly).

Based on estimates by NCBI and PIR for release CCSD8, the database, at that time, had been sent to over 600 users worldwide. The number of records in the CCSD and the publication rates of articles containing complex carbohydrate structures is growing rapidly, currently at about 12,000 records per year. CCSD14 contains over 49,000 records of complex carbohydrate sequences and corresponding bibliographic information, about 45,000 of which have been verified for accuracy.

The CCRC CarbBank staff is emphasizing software development rather than exerting an all-out effort to keep up with data entry. The Windows version of CarbBank is being enhanced, as necessary, to accommodate the continually growing CCSD. At the same time, CarbBank is being completely re-written so that it will run on the World Wide Web and thus support a wider range of computer operating systems, including Apple Macintosh and UNIX. Another major goal is to develop World Wide Web-compatible software to enable authors to submit entries directly to the CCSD via the Internet. We believe that direct author submission is the only affordable way the CCSD can be maintained as a comprehensive database. 
One update to CarbBank/CCSD was issued during this fiscal year. CarbBank was converted this year into a version capable of running on Windows 95. The Windows 95 version, which will be distributed in October 1996, can accommodate a much larger database than the previous version (currently 49,000 records) and will enable authors to prepare records for direct submission to the database as part of the manuscript submission process to publishers. This software will be beta-tested in the latter part of 1996.

*Funded collaboratively with NSF, National Library of Medicine, and National Institute of General Medical Sciences.

\section{University of Georgia Athens, GA 30602-4712}

\section{The University of Georgia Complex Carbohydrate Research Center (CCRC) P. Albersheim and A. Darvill, Complex Carbohydrate Research Center}

$\$ 910,000$

The University of Georgia Complex Carbohydrate Research Center (CCRC) has a multidisciplinary faculty and staff who serve as a national resource for basic research in complex carbohydrate science. The CCRC has nine tenured or tenure-track faculty, is recruiting two additional faculty, and expects to grow to about 14 faculty over the next five years. The grant supports research; analytical services, and training in plant and microbial complex carbohydrates. Five of the CCRC's faculty are active participants in the plant and microbial carbohydrate program supported by this grant. Educational activities involve the training of undergraduate and graduate students, postdoctoral research associates, and visiting scientists. Forty-three undergraduate and 32 graduate students are currently pursuing research projects or graduate degrees (4 M.Sc. students, 28 Ph.D. students) in the CCRC; 22 undergraduate and 7 graduate students are working in plant or microbial carbohydrate science. Fourweek-long, hands-on laboratory training courses are held annually for scientists from institutions and industries located throughout the United States and the world; 23 scientists attended these courses in 1995; 13 scientists attended the first two courses in 1996. The plant and microbial carbohydrate program has provided service to 182 individuals by analyzing 905 samples in the time that the service program has been active. These analyses include determination of glycosyl-residue and glycosyl-linkage compositions, and acquisition and interpretation of one-dimensional NMR and mass spectra. The faculty and staff of the CCRC are currently involved in more than 125 internal or external collaborative research projects of which 62 are part of the plant and microbial carbohydrate program. The CCRC has, in one way or another, assisted more than 135 corporations during the same time period; in addition, more than 100 companies were early subscribers to the Complex Carbohydrate Structure Database (when it was distributed directly from the CCRC rather than by CD-ROM or on the Internet as it is now distributed). 


\section{University of Georgia}

Athens, GA 30602-4712

\section{The Structures and Functions of Oligosaccharins $P$. Albersheim, Complex Carbohydrate Research Center}

The broad goal of this project is to determine the functions in plants of oligosaccharide signal molecules (oligosaccharins) and to learn in the process more about the interactions of plants and microbes and the regulation of plant growth and development. The importance of oligosaccharins to plants and their pathogens is made evident by the multiple mechanisms plants use to accumulate bioactive levels of these signal molecules and the corresponding mechanisms by which pathogens prevent the signal molecules from accumulating. Many of the biochemical battles that determine whether the plant overcomes or succumbs to the pathogen take place in the cell walls of plants and their microbial pathogens. Four of the six subprojects study aspects of host-pathogen interactions, emphasizing the functions of molecules of cell wall origin. These four subprojects concern: (i) plant cell wall-localized polygalacturonase-inhibitor proteins and the fungal extracellular enzymes they inhibit; (ii) fungi-secreted endo- $\beta-1,3$-glucanase-inhibitor proteins and the enzymes of the cell walls of plants that they inhibit; (iii) bioactive chitin fragments of fungal mycelial walls, the plant enzymes that generate the chitin fragments, and the enzymes of fungi that degrade the chitin fragments; and (iv) fungi-secreted endo- $\beta-1,4$-xylanases and their role in activating defense responses in Gramineae. Subproject (v) studies the bioactivities, in wild-type and mutant plants, of a plant cell wall-derived oligosaccharin that functions in regulating plant growth, and subproject (vi) centers on the isolation from plants of endogenous lipo-oligosaccharide growth regulators.

\section{University of Georgia} Athens, GA $30602-4712$

\section{Structural Studies of Complex Carbohydrates of Plant Cell Walls A. Darvill, Complex Carbohydrate Research Center \\ $\$ 415,000$}

Plant primary cell walls control several important properties of plant cells. They control the rate and direction of cell growth that determine ultimately the shapes of cells, tissues, and organs. They form a barrier to pathogens, are the source of oligosaccharins that elicit plant defense responses in host-pathogen interactions, and participate in controlling plant growth and development. Primary wall components are also used in many industrial and medical applications and thus constitute an important natural resource. The goal of this research project is to determine the structures and functions of the non-cellulosic matrix polysaccharides of primary walls that, with cellulose, account for more than $90 \%$ of the walls dry weight. The structural studies emphasize developing analytical methods and using them to elucidate the detailed structures of hemicellulosic (xyloglucan) and pectic 
(rhamnogalacturonans I and II) wall matrix polysaccharides. We are also characterizing the interactions between some of the wall matrix polysaccharides by studying the structure of two novel covalent wall cross-links. In addition, we are examining the cell-, tissue-, and species-dependent expression of cell wall epitopes using well-characterized monoclonal antibodies. This analysis may reveal developmentally and spatially regulated variations in the fine structure of the cell wall matrix polysaccharides. Finally, we are studying the structures and epitope localization of matrix polysaccharides in Arabidopsis mutants lacking or deficient in specific glycosyl residues. The long-range objective of this research is to better understand the diverse roles of the wall polysaccharides in cell wall function.

\section{University of Georgia}

\section{Athens, GA 30602-7229}

\section{Mechanisms of Lignin Biosynthesis During Xylogenesis in Zinnia elegans K-E.L. Eriksson and J.F.D. Dean, Department of Biochemistry and Molecular Biology and School of Forest Resources $\$ 89,922$}

Previous work on this project has been instrumental in showing that the expression of $\mathrm{O}_{2}$-dependent phenoloxidases is tightly associated with the lignification of vascular tissues in Z. elegans. Laccase activity extracted from Zinnia stems can be resolved into high-pl (>8.5) and low-pl $(<5.0)$ isoform classes which are composed of multiple, closely related gene products. A highly purified protein preparation containing both the high- and low-pl laccase isoforms is being used to produce monoclonal antibodies which will be used to study spatial and temporal expression the different isozymes. Isoforms purified from this pool by ion-exchange chromatography are being characterized with respect to their amino acid and glycosyl compositions, amino acid sequence, and kinetic parameters. Stem extracts from plants of varied ages show that the high-pl laccases are expressed as soon as lignified xylem vessels can be detected in the stems, but that the low-pl laccases do not become apparent until phloem and xylem fiber lignification begins. This pattern of expression is similar to that described by Varner with respect to lignin-associated Zinnia OMTs, and suggests that two differentially regulated pathways may control deposition of $G-$ and $G: S-t y p e$ lignins. A cDNA encoding a high-pl laccase isozyme has been isolated, and the gene is expressed in $48 \mathrm{hr}$ cultures of Zinnia tracheary elements. This suggests that high-pl laccases are expressed from the earliest stages of lignin deposition in protoxylem elements and vessels. A majority of the genomic laccase gene sequence, including the putative promoter region, has been cloned and is being characterized. 


\section{University of Georgia \\ Athens, GA 30602-7271}

\section{Genetic Analysis of Embryo Dormancy G.A. Galau, Department of Botany}

Primary dormancy is the ability of mature seed to germinate until specific environmental stimuli are perceived that predict that future conditions will support plant growth and seed set. Although primary dormancy impacts crop production in many ways, the results of physiological, molecular and genetic approaches to its understanding are still complex and equivocal. The approach taken here is to isolate mutants of Arabidopsis that appear to be deficient only in primary dormancy. Seed of these mutant lines appear to be deficient only in primary dormancy, germinating rapidly without the dormancy-breaking, cold stratification that is required by the parental wild-type line. All other aspects of plant and embryo growth and development appear normal in the mutant lines, suggesting that these mutants are defective only in the establishment or maintenance of primary dormancy. At least 11 RGM loci have been identified so far. In addition to further genetic analysis, the physiological phenotypes of these mutants are being evaluated, in particular the stages in embryo development at which wild-type and mutant embryos diverge.

\section{University of Georgia Athens, GA 30602-7229}

\section{Fermentation of Cellulose and Hemicelluloses by Clostridia and Anaerobic Fungi \\ L.G. Ljungdahl, Department of Biochemistry and Molecular Biology and the Center for Biological Resource Recovery \\ $\$ 163,000$}

Biomass consists mostly of cellulose, hemicelluloses, and lignin. These components are degraded in nature by consortia of anaerobic bacteria and fungi. The degradation products are fermented to methane, $\mathrm{CO}_{2}$, and organic compounds, chiefly carboxylic acids and alcohols, which can be used as industrial feedstock chemicals or alternate fuels. Bacteria and fungi secrete cellulolytic and hemicellulolytic enzymes as well as p-coumaroyl and feruloyl esterases which seem to break bonds between hemicelluloses and lignin resulting in some solubilization of the latter. Specific projects involve the structure of the cellulolytic enzyme complex the cellulosome of Clostridium thermocellum, the enzymes of the anaerobic fungus Orpinomyces PC-2, and electron transport coupled to energy generation in Clostridium thermoaceticum growing autotrophically on $\mathrm{CO}$, or $\mathrm{CO}_{2} / \mathrm{H}_{2}$. The cellulosome of $C$. thermocellum consists of 26 different polypeptides which are enzymatically active or serve as supporting proteins, scaffoldins, e.g. CipA. CipA has internally repeated domains (cohesins) which bind to conserved duplicated regions (dockerins) of the catalytically active subunits forming the cellulosome. This binding requires calcium. It is characterized together with some 
newly discovered polypeptides of the cellulosome which have cellulolytic activities or function as scaffoldins. Studies of the anaerobic fungus Orpinomyces has revealed that it produces a cellulosomal-like complex containing xylanases, endo- and exo-cellulases, and enzymes with both of these activities. It may even have esterases and if so would be able to almost completely solubilize lignocellulosic materials. The fungal "cellulosomal" complex is now being characterized using methods successfully used for the cellulosome of $C$. thermocellum. Clostridium thermoaceticum effectively ferments sugars produced by the degradation of cellulose and hemicellulose to acetate. It grows also autotrophically. Energy is generated during autotrophic growth by a coupling of the acetyl-CoA pathway with electron transport generation of ATP. The membrane of $C$. thermoaceticum contains electron transport components such as iron-sulfur proteins, b-type cytochromes, other electron carriers, and a proton dependent ATPase. The ATPase and the electron transport components are being investigated.

\section{University of Georgia Athens, GA 30602}

\section{Why Do Plants Have Two Pathways of Polyamine Synthesis? R.L. Malmberg, Botany Department}

Polyamines are small positively charged compounds, hypothesized to be involved in a wide variety of plant physiological and development functions. One feature of plant polyamine synthesis is the presence of two alternative pathways to putrescine, from arginine via arginine decarboxylase, and from ornithine via ornithine decarboxylase. We are proposing molecular and genetic analysis of the polyamine synthesis pathway in the model system Arabidopsis thaliana. The proposed research builds upon our recent isolation of mutants in the pathway and generation of molecular probes for enzymes in the pathway. There are two structural genes encoding arginine decarboxylase. We propose analysis of the regulation and function of these genes using the mutants, clones, and antibodies. Arginine decarboxylase is regulated by post-transcriptional and post-translational mechanisms; we wish to determine the mechanisms of this regulation. We will use the mutants to examine the roles of polyamines in plant stress response and in senescence. We also propose the isolation of several classes of mutants that we do not currently have, including true amorph (null) alleles and regulatory mutants. 


\section{University of Georgia Athens, GA 30602-7223}

\section{The Determinants of RNA Turnover in Higher Plants \\ R.B. Meagher, Department of Genetics $\$ \$ 198,000$ (FY 95 funds/2 years)}

Poly(A) binding proteins (PABPs) are central components of the post-transcriptional machinery and have roles affecting mRNA translation, processing, and turnover. We have shown that the model plant, Arabidopsis thaliana, has a large and ancient family of PABPs. Although plant PABPs contain the expected conserved sequence motifs, they are differentially expressed, and far more diverse in sequence than those found in other eukaryotes. Based on steady-state RNA levels and promoter/reporter fusions examined in transgenic plants, Arabidopsis PAB2 and PAB5 are specific to vascular tissues and reproductive tissues (e.g., pollen and ovules), respectively. Both bind poly $(A)$ with specificity and complement a yeast PABP mutant efficiently. In a yeast PABP mutant, PAB5 suppresses the deficiency in translational reinitiation and poly $(A)$ tail shortening and interacts with the translation factor SIS1. PAB2 did not restore poly(A) tail mediated decapping and decay of yeast mRNAs. Using a PAB2 fusion protein as a bait in a yeast dihybrid system and numerous controls to eliminate false positives, we have screened for Arabidopsis CDNAs encoding interacting prey proteins. By carefully controlling the expression levels of the bait with a carbon source, we have identified about 300 potential interacting prey from about 2 million cDNA clones. This level assures reasonable statistical completeness of the screen. Among the first prey sequenced is a known translation factor, whose interaction with PAB2 is being explored with genetic and biochemical assays. We will continue to examine the role of PABPs in post-transcriptional processes.

\section{University of Georgia}

Athens, GA 30606-7271

\section{Nitrogen Control of Chloroplast Development and Differentiation G.W. Schmidt, Department of Botany}

$\$ 96,000$

This project concerns physiological and molecular mechanisms that accompany adaptation of photosynthetic organisms to conditions of nitrogen deficiency. Analyses are performed with Chlamydomonas reinhardtii grown in a continuous culture system in which ammonium provision is restricted to limit cell division. Chloroplasts of $\mathrm{N}$-limited cells are chlorophyll-deficient but remain photosynthetically active in the synthesis of large amounts of starch and triglycerides. Chlorosis correlates with altered expression of nuclear genes encoding light-harvesting proteins, which we have shown to directly participate in synthesis of chlorophylls $a$ and $b$, as well as enzymes involved in early steps in porphyrin synthesis. The molecular basis of the nitrogen control of expression of these genes is a major thrust of this work. We also have found that respiration becomes highly sensitive to light-inhibition in 
$\mathrm{N}$-limited cells. We are pursuing evidence that the enigmatic Kok effect, formerly attributed to thylakoid chlororespiratory electron transport, is instead due to effects on mitorespiration. Nevertheless, the electron transport activities of thylakoids from $\mathrm{N}$-limited cells are altered such that the reduction of the plastoquinone pool is be sustained in darkness. A series of investigations are underway to dissect the metabolic interactions of chloroplasts and mitochondria in $\mathrm{N}$-limited cells and to further resolve the nature of the Kok effect. A related enterprise is to resolve whether there are unique or amplified mechanisms by which $\mathrm{N}$-limited cells withstand photoinhibitory conditions under high light stress.

\section{University of Georgia}

Athens, GA 30602

\section{Post-Transcriptional Regulation and Evolution of the R/B Gene Family in Maize and Rice}

S.R. Wess/er, Departments of Botany and Genetics

$\$ 104,000$

The $R / B$ genes encode a family of transcriptional activators that control the expression of the anthocyanin pathway in maize and, probably, in most flowering plants. Our analysis of $R$ comprises three projects. The first involves the use of a large collection of transposon (Ds)-induced mutations to dissect $R$ protein function. A truncated protein encoded by the $r-m 9$ allele was characterized and shown to be inefficiently localized to the nucleus due to the deletion of a nuclear localization sequence. Excision of Ds from its insertion site in the helix-loop-helix domain of the r-m1 allele led to the isolation of mutant proteins with small insertions in this important region. The second project involves understanding how a small upstream open reading frame (UORF) of 38 codons represses translation of the downstream $R$ gene. This year we made the surprising discovery that the 38 amino acids of the uORF are able to repress translation even when they are fused to the $\mathrm{N}$-terminus of the $R$ ORF. That is, the 38 amino acids can slow the flow of translating ribosomes in cis. Characterization of the $R$ gene family in rice is our third goal. This year we discovered two additional $R$ gene family members, bringing to four the number of $R$ genes in rice. The expansion of the $R$ family has occurred since the divergence of rice from other grasses indicating that the $R$ gene family has duplicated independently, frequently and recently in the grasses.

\section{University of Georgia}

Athens, GA 30602

80. Biochemistry and Genetics of Autotrophy in Methanococcus W.B. Whitman, Department of Microbiology

Even though methanogenic bacteria catalyze the terminal step in the anaerobic oxidation of organic matter, these bacteria have a very narrow substrate specificity. From this 
perspective, the carbon metabolism of the methanococci has been examined. Although these bacteria are unable to utilize exogenous sugars as carbon and energy sources, they contain the enzymes of the Embden-Meyerhof pathway for the oxidation of endogenous glycogen. In addition, the biochemical properties of the pyruvate oxidoreductase are similar to those of the oxidative enzymes common in other archaebacteria. Thus, methanococci contain the basic enzymatic machinery for the oxidation of sugars to acetate. Nevertheless, during growth on $\mathrm{H}_{2}+\mathrm{CO}_{2}$, cells do not oxidize exogenous pyruvate, and physiological mechanisms must prevent significant flow of carbon through catabolic pathways. To facilitate development of a genetic system in methanogens, the cryptic plasmid pURB500 from Methanococcus maripaludis was been sequenced in its entirety. From this plasmid, a shuttle vector was constructed by cloning in an E. coli pUC plasmid and a methanococcal selectable marker. With this vector, transformation frequencies of $4.6 \times 107 \mathrm{ug}-1$ plasmid have been obtained. This shuttle vector contains several suitable cloning sites for the addition of exogenous genes. Using the highly efficient PEG transformation method, an insertional library of $M$. maripaludis was prepared. This library contains insertional mutations in many methanococcal genes and is being used to screen for acetate auxotrophs.

\section{University of Georgia Tifton, GA 31793}

\section{Development of Innovative Techniques That May be Used as Models to Improve Plant Performance \\ W.W. Hanna and G.W. Burton, Department of Agronomy \\ $\$ 55,000$}

The objectives of this project are to: (1) establish the cytoplasmic diversity of germplasm in the weedy subspecies of the primary gene pool and demonstrate its value, (2) identify mechanism(s) for transfer of germplasm from the secondary gene pool to the cultivated species and evaluate the plant breeding potential of this germplasm, and (3) transfer gene(s) controlling apomixis from the tertiary gene pool to cultivated pearl millet for the purpose of producing true-breeding hybrids. Species within the genus Pennisetum are being used as test organisms. The approach uses plants of wild species with different genetic and cytoplasmic backgrounds, ploidy levels, and chromosome numbers crossed and backcrossed with different genotypes of diploid and tetraploid pearl millet. A new cytoplasm from the primary gene pool was identified from two-year forage yield trials that produced $17 \%$ more dry matter in hybrids than the standard commercial A1 cytoplasm. Genotypes were identified among cultivated $x$ secondary gene pool species crosses which showed preferential segregation for $A^{\prime}$ genome chromosomes from the wild species. Apomictic BC6 plants resembling cultivated pearl millet were identified that produced up to $96 \%$ maternal types. 


\section{University of Hawaii Honolulu, HI 96822}

\section{Violaxanthin De-epoxidase: Biogenesis and Structure H.Y. Yamamoto, Department of Plant Molecular Physiology \\ $\$ 120,000$}

Violaxanthin de-epoxidase (VDE) is an enzyme located in the lumen of thylakoids that converts violaxanthin to antheraxanthin and zeaxanthin in the presence of ascorbate and an acidic lumen. The presence of zeaxanthin and antheraxanthin, along with the transthylakoid delta $\mathrm{pH}$, induces non-radiative dissipation of energy in the antennae, decreasing quantum yield of PSII. VDE as part of this feed-back regulatory mechanism, helps to keep PSII traps open, functioning, and protected against photoinhibition. The cloning of lettuce VDE was previously reported. Using probes generated from lettuce VDE, the CDNA of tobacco and Arabidopsis were cloned and sequenced. The deduced mature proteins show high degrees of homology, having a hydrophobic and cysteine rich $\mathrm{N}$-terminus, a lipocalin signature near the cysteine-rich region, and a glutamic-acid rich $\mathrm{C}$-terminus. Tobacco was transformed with sense and antisense constructs using Agrobacterium. About forty plants of each construct (including controls) were grown to seed. Analyses of these transformed plants for violaxanthin de-epoxidase activity and non-photochemical quenching are underway.

\section{University of Illinois Chicago, IL 60612}

\section{Heavy Metal-lux Sensor Fusions and Gene Regulation S. Silver, Department of Microbiology and Immunology}

$\$ 105,000$ (FY 95 funds/15 months)

We are studying the molecular genetics of bacterial resistances to the toxic heavy metals, mercury, cadmium, arsenic and silver. Bacterial cells have highly specific genetic systems for each of these toxic minerals. Each system contains gene(s) for regulation (so that the system is functional only when needed) and gene(s) for the proteins that physiologically and biochemically confer resistance itself. The resistance systems offer promise for "bioremediation" of environmentally polluted settings, while the regulatory genes may be used as components of metal-specific "biosensors" (especially with luciferase gene fusions that respond to "bioavailable" metal ions with increasing light emission). Mercury and methyimercury-detoxifying Bacillus isolates from Minamata Bay Japan will be analyzed to follow the relationship between detailed laboratory studies with new environmental isolates. The newly recognized CadC, transcriptional repressor of the cadmium-resistance cadmium-efflux ATPase will be studied by protein-DNA interactions in vitro and in vivo. Mutants with altered CadC repressor will be used to test specific hypotheses concerning metal-protein binding and protein-DNA binding. The enzymatic transformations of arsenic by the enzymes arsenate reductase and arsenite oxidase hold potential for microbial 
bioremediation of soil and water arsenic pollution. The first silver-resistance system has just been cloned and will be sequenced during the next year, along with gene products identified and characterized.

\section{University of Illinois}

\section{Urbana, IL 61801}

\section{Studies on the $\mathrm{bo}_{3}$-type Ubiquinol Oxidase from Escherichia coli R.B. Gennis, Department of Biochemistry}

$\$ 125,000$

The $\mathrm{bo}_{3}$-type ubiquinol oxidase is the predominant respiratory oxidase present in the bacterial membrane of Escherichia coli when the cells are grown with high aeration. This enzyme, also known as the cytochrome o complex, is a coupling site. This means that the redox reaction catalyzed by the enzyme, i.e., the 4-electron reduction of oxygen to water, is coupled to the generation of a proton and voltage gradient across the cytoplasmic membrane. Our primary interest is to learn how this is done.

Our studies have established this enzyme as a member of the superfamily of heme-copper respiratory oxidases. This superfamily includes the eukaryotic aa3-type cytochrome c oxidases. The focus of our work is to combine site-directed mutagenesis with a variety of spectroscopic techniques to identify amino acid residues that are structurally and/or functionally important. Based on these studies, we built models of the enzyme which have largely been confirmed by the recent high resolution $\mathrm{X}$-ray structures announced recently for two cytochrome c oxidases.

Current efforts are directed at using the mutant oxidase species to examine the catalytic mechanism. We are especially interested in the role of the two distinct proton channels.

\section{University of lllinois Urbana, IL 61801}

\section{Regulation of Cell Division in Higher Plants T. Jacobs, Department of Plant Biology $\$ \$ 94,000$ (FY 95 funds/20 months)}

Plant cells divide not to generate morphogenetic building blocks, but to partition space within the expanding organism. The developmental role of mitosis in plants thus differs from that in animals. The ultimate aim of this project is to identify the biochemical mechanisms by which angiosperm morphogenesis drives mitosis. Our strategy begins with plant cyclins and cyclin dependent kinases (CDKs) and works outward from these conserved regulators of the cell cycle engine in search of their functional links to cellular physiology and developmental processes. We work on two complementary fronts. First, we are pursuing the mechanisms 
underlying mitotic retirement in cell lineages of the pea seedling root. We are testing several hypotheses arising from our observation that unknown factors extractable from the basal cells of this organ's terminal $5 \mathrm{~cm}$ inhibit CDK activity purified from mitotic apical cells. Second, we are continuing to develop and explore new biochemical tools to fuel our efforts on the first front. For example, we have used interaction cloning to identify cellular proteins with which plant CDKs and their regulators interact. These efforts have yielded, among others, two polypeptides to which a pea CDK binds in vitro, both of which are components of the cell's protein synthesis machinery. We have also verified that an Arabidopsis homologue of a protein that interacts with $\mathrm{Rb}$, the retinoblastoma tumor suppressor protein, also binds $\mathrm{Rb}$. Upcoming experiments will explore the nature of these associations in vivo and the linkage between protein synthesis and the cell cycle.

\section{University of Illinois Urbana, IL 61801}

\section{Exploratory Studies on the Bacterial Formation of Methane} R.S. Wolfe, Department of Microbiology

The microbial formation of methane is carried out by a unique group of bacteria known as methanogens. These strict anaerobes are widespread in nature, and are found in diverse habitats, wherever active anaerobic degradation of organic matter occurs, such as sediments, the rumen of ruminants, sanitary landfills, and sewage sludge digesters. We are exploring ways of simplifying the culture of methanogens in liquid and solid media, so that these organisms may be more readily employed as research tools by the scientific community. These studies include the sensitivity of methanogens to a variety of factors such as reducing agents, ions, ionic strength, and oxidizing agents. For example, under certain conditions of stress, cells of methanogens may exhibit a bright red fluorescence instead of the typical blue-green fluorescence. We are studying this phenomenon to isolate and characterize the red fluorescent compound, to determine its structure, its role, and its relationship to known coenzymes which may be modified when cells are under stress. Our goal is to define how sensitive, anaerobic methanogenic-cells survive under stress in terms of biochemistry. 


\section{University of Illinois}

Urbana, IL 61801

\section{Integrative Photosynthesis Research C.A. Wraight, D.R. Bush, J.M. Cheeseman, A.R. Crofts, P.G. Debrunner, E.H. DeLucia, Govindjee, D.R. Ort, A.R. Portis, J. Whitmarsh, and R.E. Zielinski}

This interdisciplinary research training program focusses on factors influencing the photosynthetic performance and productivity of plants. The role of environmental and anthropogenic factors is further explored through the academic curriculum and seminars. Many of the most significant issues span the range from physics to agronomy and an interdisciplinary outlook and multidisciplinary training are especially important for future research. To acquaint students with the broad knowledge base and the experimental skills required to address these complexissues, graduate students and young postdoctoral trainees work on projects coordinated between laboratories of the training program faculty, providing conceptually different outlooks and approaches to a problem. Several research projects are examining responses to excess irradiance and the interaction with other environmental stresses such as temperature extremes and drought. Other research areas include the metabolism of oxidative byproducts, responses to UV-B irradiation, the role of the circadian rhythm in chilling sensitivity, and the in situ regulation of photosynthetic enzymes, including the thylakoid ATPase, rubisco and rubisco activase, using genetic and antisense RNA methods. Emphasis is placed on assessing photosynthetic performance under natural conditions and many projects include field measurements of photosynthetic functions, using locally-designed, sophisticated but portable measuring devices. Undergraduate students are brought into the research environment through summer fellowships for research in faculty labs. A summerworkshop on Photosynthesis and Global Climate Change for high school and community college teachers is designed to present this topic as a paradigm of the relationship between modern research and societally important issues.

\section{lowa State University}

\section{Ames, IA 50011}

\section{Characterization of the Membrane-Associated Methane Oxidation System in} Methylococcus capsulatus Bath

A.A. DiSpirito, Department of Microbiology, Immunology and Preventive Medicine

$\$ 94,000$

Methanotropic bacteria are characterized by the ability to utilize methane as a sole carbon and energy source. The oxidation of methane to methanol in these microorganisms involves a series of two electron steps with methanol, formaldehyde and formate as intermediates. The first enzyme in the pathway, methane monooxygenase (MMO), catalyzes the energy 
dependent oxidation of methane to methanol. In some methanotrophs the cellular location of the MMO has been shown to depend on the copper concentration during growth. At low copper to biomass ratios, the enzyme activity is observed in the soluble fraction and referred to as the soluble MMO (sMMO). At higher copper to biomass ratios, methane oxidation activity is observed in the membrane fraction and the enzyme referred to as the membraneassociated or particulate MMO (pMMO). We have recently isolated the pMMO. The enzyme consists of three polypeptides with molecular masses of 47,000,27,000, and 25,000 Da, contains 2.5 non-heme iron and 14.6 copper atoms per 99,000 Da. The majority, 10 to 12 of the copper atoms, are associated with a low molecular weight (1,236 Da) copper bind cofactor (cbc).

The overall objective of this project is to characterize the mechanism of methane oxidation in methanotrophs expressing the pMMO. Present work is focused on improvements to the pMMO purification procedure, the characterization (physical, spectral and enzymatic) of the pMMO, and the characterization of the cbc. Work is also in progress to determine the physiological reductant of the pMMO.

\section{lowa State University}

Ames, IA 50011-1020

\section{Regulation of Carotenoid Biosynthesis: The Immutans Variegation Mutant of Arabidopsis \\ S. Rodermel and D. Voytas, Department of Botany and Department of Zoology and Genetics

The immutans (im) variegation mutant of Arabidopsis thaliana contains green- and white-sectored leaves due to the action of a nuclear recessive gene. The somatic instability of the mutant is influenced by light, temperature and developmental factors. The white sectors of im are heteroplastidic for normal and abnormal, non-pigmented plastids (i.e., im expression is "plastid autonomous"), but the defective plastids are not maternally-inherited, as in other cases of variegated plants with heteroplastidic cells. im is thus a novel type of nuclear gene-induced variegation mutant. Phytoene, a carotenoid intermediate, accumulates in the im white sectors, indicating that im controls the activity of phytoene desaturase (PDS), the enzyme that converts phytoene to zeta-carotene. However, im is not the structural gene for P.DS, nor does im affect PDS mRNA or PDS protein levels--i.e., im is not PDS or a factor required for PDS expression. Fluorescence activated cell sorting (FACS) of pure-white protoplasts from transgenic im plants that express an Lhcb promoter/GUS fusion showed that Lhcb transcription is much higher in these cells than in norflurazon-treated (photooxidized) white cells from control plants. This suggests that im partially uncouples Lhcb transcription from its normal dependence on chloroplast development. To further characterize the primary lesion in im plants, we are cloning immutans by positional cloning procedures, and to date, have localized im within a $30 \mathrm{~kb}$ DNA fragment. We conclude that im is an important component in signal transduction pathways that control chloroplast biogenesis. 


\section{University of lowa lowa City, IA 52242}

\section{Molecular Biology of Anaerobic Aromatic Biodegradation \\ C.S. Harwood, Department of Microbiology}

We have been studying the molecular basis for anaerobic aromatic compound degradation using the phototrophic bacterium Rhodopseudomonas palustris as a model organism. Emphasis has been on the compounds benzoate and 4-hydroxybenzoate, since these aromatic acids are formed as major breakdown products of plant material and as intermediates in the degradation of many environmental pollutants. They are also the starting compounds for a central pathway of anaerobic benzene ring reduction and ring fission. We have recently cloned and sequenced a $30 \mathrm{~kb}$ region of the $R$. palustris chromosome that appears to include all the genes required for anaerobic benzoate and 4-hydroxybenzoate degradation. Our goal is to define the role of each gene product in degradation. This should aid in the elucidation of the precise sequence of enzymatic reactions in the degradation pathways. The details of novel enzymatic features of anaerobic degradation, a process carried out only by prokaryotes, should also be revealed. In addition, cloned genes will be used to determine how gene expression is regulated in response to aromatic compounds and oxygen. This information will be valuable in designing bacterial systems to mediate biomass conversions or for use in bioremediation.

\section{Johns Hopkins University Baltimore, MD 21218}

\section{Bacterial Nickel Metabolism and Storage R.J. Maier, Department of Biology}

$\$ 102,000$

Nickel is an essential element for the growth of many microorganisms, as nickel is a component of several microbial enzymes. From studies on bacterial mutants in Ni-containing ureases and hydrogenases, it has recently become clear that the Ni-requiring organisms contain enzymes that deal with intracellular nickel metabolism and subsequent insertion into the Ni-enzyme(s). To determine the routes of nickel metabolism and incorporation into a bacterial enzyme (hydrogenase) in the $\mathrm{N}_{2}$-fixing bacterium Bradyrhizobium japonicum gene-directed mutant strains of the bacterium have been studied. Some mutant strains are specifically deficient within histidine rich areas of the protein we call nickelin. Analysis of the mutants within the metal-binding domain are suggestive that the his-rich area, capable of binding 18 nickel atoms per molecule of (HypB) protein plays a nickel storage role for subsequent hydrogenase expression. Other proteins involved with the nickel-dependent regulation of hydrogenase expression either in sensing nickel or in activating hydrogenase transcription have been identified and specific mutants in each of the genes have been generated. Some of the genes encode domains which act as nickel-binding ligands, and a 
nickel dependent sensing and regulatory cascade for Ni-enzyme (hydrogenase) expression involving at least six proteins can be proposed. The long-term goal is to understand the number, nature, and function of the components involved in bacterial nickel metabolism.

\section{Johns Hopkins University Baltimore, MD 21218}

\section{Transport of Ions Across the Inner Envelope Membrane of Chloroplasts R.E. McCarty, Department of Biology $\$ 103,000$}

The reduction of nitrate to ammonia in leaves takes place in two stages. Nitrate is reduced to nitrite in the cytoplasm whereas the reduction of nitrite to ammonia takes place in chloroplasts. How nitrite crosses the inner envelope membrane, the permeability barrier of the chloroplast, has been a controversial subject. Based on studies with instruct chloroplasts, diffusion of nitrous acid and mediated transport have been proposed. Inner envelope vesicles were loaded with the $\mathrm{pH}$ indicator, pyranine, by extrusion of the vesicles through $100 \mathrm{~nm}$ polycarbonate membranes. Very rapid acidification of the interior of the vesicles was observed when the vesicles were mixed in a stopped-flow apparatus with sodium nitrite. The rate of acidification was a linear function of the nitrite concentration and increased as the external $\mathrm{pH}$ decreased. Nitrite induced acidification was insensitive to $\mathrm{N}$-ethylmaleimide. These results are consistent with nitrous acid diffusion being the mechanism of the rapid acidification. In accordance with this idea, the properties of nitrite transport in vesicles composed of soybean lipids were found to be very similar to those inner envelope vesicles. The rate of nitrous acid transport in the inner envelope vesicles was estimated to be at least five times that of the highest rate of nitrite reduction in intract chloroplasts. Although these results show that nitrous acid diffusion across the envelope is fast, they do not include the existence of another transport mechanism for nitrite; for example, nitrite could be transported through an anion channel.

\section{KAIROS Scientific Inc.}

\section{Santa Clara, CA 95054}

\section{Macromolecular Scaffolds for Energy Transfers}

D.C. Youvan

$\$ 174,867$ (16.5 months)

If emerging studies prove correct, the Green Fluorescent Protein (GFP) from the jellyfish Aequorea victoria is the first known example of a protein that utilizes a Forster cycle. The recent X-ray crystallographic structure of GFP, along with our collaborative studies on mutants with Prof. Michel-Beyerle of T.U. Munich, suggest that GFP's chromophore can be excited to a species $\mathrm{RH}^{*}$ which forms the excited anion $\mathrm{R}^{* *}$ by a classic photochemistry mechanism discovered by Forster half a century ago for phenolic compounds. Although there are several 
sites on the chromophore which could be involved in excited state proton transfer, the new $X$-ray structure might offer a key to a qualitative and quantitative understanding of the role of the tyrosine on the chromophore and an adjacent histidine in the protein with its inherent proton donor/acceptor properties. Indeed, the structural data suggests that GIn94 / Arg96 and His 148 could participate in a "push-pull" mechanism for the proton translocation by stabilizing resonant forms of the ionized tyrosine and imidizolidone on the fluorophore, respectively. These amino acid residues are currently targeted for site-directed and combinatorial mutagenesis in order to further elucidate this novel mechanism. In terms of energy studies, the Forster mechanism is an efficient means to shift the wavelength of light $100 \mathrm{~nm}$ from violet absorption to green emission. Since this fluorophore forms through an autocatalytic mechanism on the apoprotein's backbone (i.e., no cofactors or biosynthetic enzymes are involved), protein engineering can be used to modify the Forster cycle within this macromolecule.

\section{University of Kentucky Lexington, KY 40536-0084}

\section{Acetyl-CoA Cleavage and Synthesis in Methanogens: Mechanistic, Enzymological, and Metabolic Studies \\ E. DeMoll, Department of Microbiology and Immunology; D.A. Grahame, Uniformed Services University of the Health Sciences $\$ 94,000$}

We are studying the biochemistry and physiology associated with acetyl-CoA synthesis and cleavage in methanogens. This reversible reaction is catalyzed by the acetyl-CoA decarbonylase/synthase (ACDS) complex. We have described three partial reactions carried out by protein subcomponents of the complex, and are presently using the results from those studies to help us determine the kinetic mechanisms involved in the various biochemical steps. We are also testing our hypothesis that in methanogens growing with $\mathrm{C} 1$ units as sole carbon and energy source, carbon flow through the ACDS complex is restricted by the level of available reducing equivalents. This growth restriction cannot take place in methanogens that are growing with acetate as sole carbon and energy source, so we are determining how acetyl-COA cleavage causes an activation of the ACDS complex: 


\section{University of Kentucky Lexington, KY 40546-0091}

\section{Mechanism and Significance of Post-Translational Modifications in the Large Subunit of Ribulose-Bisphosphate Carboxylase/Oxygenase \\ R.L. Houtz, Department of Horticulture and Landscape Architecture $\$ 88,705$ (FY 95 funds/18 months)}

A biochemically important and prevalent plant protein which is methylated in many but not all plant species is ribulose-1,5-bisphosphate carboxylase/oxygenase (Rubisco). Our research is focused on defining the enzymological properties, molecular structure, and functional significance of the protein (lysine) $\mathrm{N}$-methyltransferase responsible for the site-specific methylation of Lys-14 in the large subunit (LS) of Rubisco (Rubisco LSMT). Our most recent efforts have resulted in the isolation and characterization of the Rubisco LSMT gene (rbcMT-T) from tobacco. The tobacco rbcMT-T gene is present as a single copy in the tobacco haploid genome, contains 6 exons, and spans approximately 6 kilobase pairs. The full-length CDNA for tobacco Rubisco LSMT encodes a 491 amino acid polypeptide and has been engineered into transgenic tobacco plants in the sense and antisense orientations in order to examine the in vivo functional significance of Lys-14 methylation. Additional and related studies have been focused on the molecular rationale for the absence of Lys-14 methylation in spinach, identification of the S-adenosyl-L-methionine binding site and determinants of the highly specific and tight interaction between Rubisco LSMT and des(methyl) forms of Rubisco, and a kinetic characterization of the reaction mechanism for Rubisco LSMT. The results from these studies suggest that a related but functionally distinct Rubisco LSMT gene exist in spinach, that the LS is the major determinant for binding of Rubisco LSMT with at least four binding sites per Rubisco holoenzyme, and that the reaction mechanism may be a hybrid ping-pong mechanism.

\section{University of Kentucky \\ Lexington, KY 40546-0091}

\section{Studies of a Novel Pathway for Biosynthesis of Straight and Branched, Odd and Even Length, Medium-Chain Fatty Acids in Plants.

Fatty acids (FA's) are key components of membranes, waxes, etc. that are essential to plant growth and development. They constitute the main energy reserve for seed germination and seedling development in certain oil seeds. Oil seeds are extensively exploited commercially for their food value and as lubricants, emulsifiers, etc.

Metabolism leading to branched and odd carbon number FA's is little understood. We have described a new pathway (termed alpha keto acid elongation-a KAE) that appears to utilize 
enzymes of branched chain amino acid biosynthesis to form short and medium chain FA's in trichome glands. We recently determined that this pathway is not involved in medium chain fatty acid biosynthesis in Cuphea and coconut seed or in long chain fatty acid formation in these plants or in soybean, rape or Arabidopsis seeds or leaves. Preliminary experiments indicate that (-KAE is not involved in formation of branched or straight chain acid components of wax esters in broccoli or tobacco. Key enzymes involved in a-KAE will be isolated and studied. Isolation of their genes will begin in an effort to use genetic engineering to attempt modification of FA and wax ester composition. Yeast and human CDNA's for two key enzymes involved in a-KAE metabolism will be introduced into the model plant tobacco to assess the feasibility of genetic engineering for modification of FA components of trichome-exudate sugar esters. Trichome exudate of certain highly-exudated, high-biomass plants represents an unexploited, renewable resource for production of medium and short chain FA's having various configurations and structures.

\section{Lawrence Berkeley National Laboratory Berkeley, CA 94720}

\section{Enzymatic Synthesis and Biomolecular Materials \\ M.D. Alper, C. Bertozzi, D. Charych, J.F. Kirsch, D.E. Koshland, P.G. Schultz, R. Stevens, F. Tropper and C.-H. Wong, Center for Advanced Materials \\ $\$ 160,000^{*}$}

The goal of this research is the use of natural biological processes and molecules as the basis for the synthesis of new materials.

One component focuses on the use of natural, engineered and "created" enzymes to synthesize new materials. The unique stereochemical control exerted by enzymes and their ability to catalyze reactions at low temperature allows the synthesis of materials with structures and therefore properties that cannot be achieved using conventional synthetic routes. Efforts are focused on the design of reaction conditions for the enzymatic synthesis of polymeric materials; engineering of enzyme structure and activity to allow the binding and polymerization of novel monomers; generation of catalytic antibodies for materials synthesis; characterization and processing of the polymer products of these reactions and understanding the structure/function relationships of this new class of materials. Other polymers with structures inspired by biological polymers are being synthesized chemically.

Work is also progressing on the synthesis of organic thin films which mimic the biological membrane to alter interfacial and surface properties, and to fabricate sensor devices. Thin film sensors have been developed to detect influenza virus, botulism and cholera toxins. Similar films have been used to direct the ordered crystallization of inorganic salts. 
Research is also focused on the modification of materials' surfaces to improve their biocompatibility.

* Funded jointly with the Division of Materials Sciences (DOE).

\section{Lawrence Berkeley National Laboratory Berkeley, California 94720}

\section{Structural Studies of Plant Signal Transduction Proteins}

A. Grantz, W. Gruissem, and S.-H. Kim, Structural Biology Division and Department of Plant and Microbial Biology, University of California, Berkeley

In contrast to the active and extensive studies of many signal transduction pathways in mammalian and bacterial systems, the signal transduction pathways in plant systems are just beginning to be elucidated. The overall objective of this project is to determine the threedimensional structures of several proteins associated with the signal transduction pathways of ethylene and plant development. Currently we are focusing our attention to ethylene receptor and farnesyltransferase. As for the former, we have subcloned the response regulator domain of ETR-1, an ethylene receptor from Arabidopsis. After expression of the gene with an His-tag and purification of the expressed protein in a metal column, we removed the His-tag with Factor $X_{a}$. Final purification was done on a Superdex 75 column. We have now crystallized the protein and the crystallographic studies are in progress. We have also recently over-expressed the histidine kinase containing domain of ETR1 as the Glutathione-Stransferase (GST) fusion proteins. As for the farnesyltransferase we have cloned and expressed both subunits of tomato farnesyltransferase. Over-expression and purification of these proteins are in progress. Once successful, we will initiate crystallization experiments and crystallographic studies of the proteins.

\section{Lawrence Berkeley National Laboratory Berkeley, CA 94720}

\section{Transferring Photosynthesis Genes to New Cellular Environments J.E. Hearst, Structural Biology Division

We have sought a genetic and molecular understanding of photosynthetic processes both in bacteria and in plants. In the past our work has centered on the photosynthetic bacterium Rhodobactercapsulatus. This bacterium contains a single chromosomal region, 46 kilobases $(\mathrm{kb})$ in length, which codes for all of the essential genes for photosynthesis. This cluster includes the genes for the reaction center $(\mathrm{RC})$ proteins, a long wavelength light harvesting antenna (LHI), and the enzymes for the biosynthesis of bacteriochlorophyll (Bchl) and 
carotenoid (Crt) pigments. Work on the mapping of this gene cluster is complete, as is our work on a second gene cluster which contains all of the genes required to make Crt pigments in the non-photosynthetic bacterium Enwinia herbicola. The complete nucleotide sequences of each cluster have been determined, denoting a landmark in the molecular biological studies of photosynthesis. Making use of what we have learned from each of these two systems, we are now directing our efforts toward the genetic transfer of pigment biosynthesis and photosynthesis itself to organisms which are now incapable of performing these functions. The long term goals of our task are: i) to gain an understanding of the evolutionary steps which took place in the transfer of photosynthesis to plants, ii) to use the information and tools developed during studies on these gene clusters to gain some understanding of the photosynthetic processes in plants, iii) to use the genetically engineering constructs which are now available to transfer biosynthetic enzyme activity into new cellular environments.

\section{Lawrence Berkeley National Laboratory Berkeley, CA 94720}

\section{Mechanism of Water Splitting and Oxygen Evolution in Photosynthesis M.P. Klein and V.K. Yachandra, Structural Biology Division ! $\$ \mathbf{\$ 2 1 5 , 0 0 0}$}

Photosynthetic oxygen evolution in plants and cyanobacteria is catalyzed by a cluster of four Manganese atoms. The Mn cluster is the site of water splitting and acts as the locus of charge accumulation that is driven by the energy of four successive photons. The oxidation states of the Mn in four (of the five) of these intermediate or S-states have been determined from Mn K-edge X-ray absorption spectra, for samples prepared by microsecond flashes at room temperature and characterized for S-state composition by EPR. Oxidation of Mn accompanies the first flash from the dark stable $S_{1}$ to the $S_{2}$ state, little or no change in oxidation is induced by the second flash that induces the $S_{3}$ state and sizable reduction follows the third flash leading to the $S_{4}-S_{0}$ states. The fourth and fifth flashes restore the $S_{1}$ and $S_{2}$ states. The structure of this cluster in the $S_{1}$ and $S_{2}$ states, as determined by EXAFS, is consistent with a pair of di- $\mu$-oxo bridged $M n$ dimers with $M n-M n$ distances of $2.72 \AA$ and $2.85 \AA$ linked by a mono- $\mu-0 x o$ bridge with $M n-M n$ distance of $3.3 \AA$. Replacement of $\mathrm{Ca}$ with $\mathrm{Sr}$ establishes that $\mathrm{Sr}(\mathrm{Ca})$ is within $3.5 \AA$ of the $\mathrm{Mn}$ cluster. EXAFS of the $\mathrm{S}_{3}$ state suggests a significant structural change. In turn, this leads us to propose a new mechanism for formation of the $\mathrm{O}-\mathrm{O}$ bond. 


\section{Lawrence Berkeley National Laboratory Berkeley, CA 94720}

\section{Photosynthetic Pigment Proteins and Photosynthetic Light Reactions K. Sauer, Structural Biology Division

The Pebble Mosaic model for light absorption in antenna pigment-proteins, followed by excitation transfer and trapping in reaction centers of photosynthetic membranes has been tested on two antenna pigment protein complexes of known structure. Using fast timeresolved fluorescence measurements, we have characterized the rapid and extensive excitation transfer in the pigment-protein C-phycocyanin (C-PC). Using the X-ray crystallographic structure for $\mathrm{C}-\mathrm{PC}$ we have obtained excellent agreement between theoretical predictions and our time-resolved fluorescence relaxation measurements for C-PC monomers and trimers, showing that the Förster mechanism is sufficient to explain the observed behavior. By contrast, the excitation transfer dynamics in the $\mathrm{LH} 2$ bacteriochlorophyll (BChl)protein complex from purple photosynthetic bacteria is dominated by excitonic interactions among the $\mathrm{BChl}$ molecules in $\mathrm{LH} 2$. These results have been published, in collaboration with colleagues from the University of Glasgow who determined the structure of this pigmentprotein in 1995, and some of our predictions of spectroscopic and relaxation properties are now undergoing investigation.

The light reaction of Photosystem 2 of higher plants and cyanobacteria results in the oxidation of water to molecular oxygen. We have made extensive use of EPR and X-ray absorption spectroscopy to postulate a structure for the manganese-containing complex that carries out this process. We are currently using different infrared absorption spectroscopy to investigate the mechanism of water oxidation, and especially the steps involved in proton removal and formation of the $\mathrm{O}-\mathrm{O}$ bond. For model compounds of known structure we have identified lowfrequency $\left(400-800 \mathrm{~cm}^{-1}\right)$ vibrations that are characteristic of multi-nuclear $\mathrm{Mn}$ complexes with oxo-bridges. Using difference spectra between samples in different $\mathrm{S}$-states and using $\mathrm{H}$ and $O$ isotope replacement, we will characterize the structural changes that accompany water oxidation and $\mathrm{O}_{2}$ formation by the $\mathrm{Mn}$ complex in this essential photosynthetic energyconverting reaction.

\section{Los Alamos National Laboratory}

\section{Los Alamos, NM 87545}

102. Carbon Metabolism in Methylotrophic Bacteria C.J. Unkefer, Chemical Science and Technology Division

The purpose of this project is to understand the unique aspects of the metabolism and biochemistry of methylotrophic bacteria which grow on reduced one carbon compounds such as methane, methanol, or methylamine. Methylotrophs possess a unique system for oxidizing 
alcohols which involves the PQQ-dependent methanol dehydrogenase (MDH). The MDH oxidation of alcohols is coupled directly to the electron transport chain, and is used to drive the net synthesis of ATP. When growing on methane or methanol, methylotrophs derive all of their energy from this process. The MDH cofactor PQQ (pyrroloquinoline quinone) is one of the three o-quinone enzyme cofactors that have been identified. $P Q Q$ is unique among this group in that it must be biosynthesized as distinct molecule. Using ${ }^{13} \mathrm{C}$ labeling and NMR spectroscopy have shown that $P Q Q$ is biosynthesized from the amino acids glutamate and tyrosine. We are continuing to probe the biosynthesis of $P Q Q$. Molecular genetic studies in other laboratories have identified PQQ biosynthesis genes. All three organisms studied contain an open reading frame that encodes for a 23-29 amino acid peptide. We are testing the hypothesis that this peptide is the precursor for the biosynthesis $P Q Q$. We are currently synthesizing the Methylobacterium extorquens AM1 peptide labeled with $L-\left[3^{\prime}-5^{\prime}-{ }^{13} \mathrm{C}_{2}\right]$ tyrosine and will examine extracts for their ability to oxidize this peptide. During our PQQ biosynthesis studies we generated ${ }^{13} \mathrm{C}$-labeled samples of $\mathrm{PQQ}$ which were used to probe the mechanism of $\mathrm{MDH}$. Using NMR spectroscopy we demonstrated that upon formation of the enzyme-substrate complex the alcohol binds to the C-5 carbonyl of PQQ to form a hemi-ketal structure. In addition, when cyanide, a competitive inhibitor of MDH binds reversibly to $P Q Q$ as a cyanohydrine at the C-5 carbonyl. The ability of methylotrophs to grow on $\mathrm{C}_{1}$ compounds gives them considerable industrial potential because they could be used to produce a variety of useful compounds from inexpensive and renewable precursors such as methanol or methane. Their ability to oxidize a wide variety of chemicals make them potentially important for treatment of waste streams. For the potential of these organisms to be realized, we must understand fundamental aspects of the their physiology.

\section{University of Maryland Baltimore, MD 21202}

\section{Structure and Regulation of L-glutamate Dehydrogenase in Hyperthermophilic Archaea \\ F.T. Robb and J. DiRuggiero, Center of Marine Biotechnology $\$ 97,072$}

Glutamate dehydrogenase is an enzyme which is assumed to play a prominent part in central nitrogen metabolism of hyperthermophilic Archaea. Comparative studies between the GDHs from mesophiles and hyperthermophiles have focussed on the importance of ion pair formation as a prominent structural feature of enzymes from hyperthermophiles, while the presence of internal packing appears to be enhanced to ensure internal rigidity and thus enhance stability in hyperthermophiles. It is our objective to substantiate these two hypotheses. The sequence and crystal structure of the extremely thermostable Pyrococcus furiosus GDH was used to identify five residues in the homologous, but five-fold less thermostable GDH from Thermococcus litoralis, with possible roles as determinants of thermostability. The $T$. litoralis gdhA gene was mutated and the mutant genes were expressed in $E$. coli, resulting in enzymes with specific activity comparable to the wild type, recombinant GDH. Three initial mutations (Mut1:T138E; Mut2:T138E, V127I; Mut3:T138E, 
V127I, V172I) resulted in drastically reduced thermostability and mixed results by scanning microcalorimetry. The final two mutations D167T and S169A, added to the previous three mutations, resulted in a 3-4-fold elevation of thermostability at $98^{\circ} \mathrm{C}$, and a substantial upward shift of the $T_{m}$ determined by scanning microcalorimetry, from $104^{\circ} \mathrm{C}$ to $111^{\circ} \mathrm{C}$. The altered $T$. litoralis $\mathrm{GDH}$ is very close to the previous upper limit reported for dehydrogenases, namely $P$. furiosus $\mathrm{GDH}$ with a $\mathrm{T}_{m}$ of $113^{\circ} \mathrm{C}$. Our work demonstrates the usefulness of a combined genetic and structural approach towards determining the structural basis for extreme protein thermostability.

\section{University of Maryland Baltimore, MD 21202}

\section{Regulation of Catabolic Gene Transcription in the Methanogenic Archaea K.R. Sowers, Center of Marine Biotechnology $\$ 182,000$ (2 years)}

The controlled expression of catabolic gene products is integral to microbial interactions that mediate biomass conversion to methane. The goal of this project is to determine the mechanisms of transcriptional gene regulation in the methanogenic Archaea which are the catalysts of methane production and have a role in global carbon cycling. Although the Archaea have structural gene characteristics that are similar to those of both the Bacteria and the Eucarya, preliminary evidence suggests that they employ Eucarya-like transcription factors for site-specific transcription initiation. We have shown that transcription of the gene encoding CO dehydrogenase (cdh) from Methanosarcina thermophila is highly regulated in response to substrate. An in vitro transcription assay has been developed using a template that contains the promoter and 300 base pairs of the $5^{\prime}$ end of $\operatorname{cdhA}$, and the lac terminator sequence. The in vitro assay has been optimized and we have shown $>20$-fold expression of cdh' mRNA expression with cell-free extracts of acetate-grown cells compared with methanol grown cells. Concurrently, a transformation protocol has been developed for Methanosarcina spp. and a simple, inexpensive anaerobic plating vessel has been developed that provides a virtually inexhaustible source of growth vessels for colony selection of genetic transformants. A plasmid expression vector is being constructed that will enable us to measure cdh transcription activity in vivo. Results of this study will determine whether gene regulation in the Archaea functions by mechanisms that are analogous to the other two lineages or by mechanisms that are unique to this phylogenetic line. Regardless of which mechanism(s) is revealed by this investigation, the results will provide further insight into the global molecular strategies of gene regulation. 


\section{University of Maryland College Park, MD 20742}

\section{Structure and Regulation of Calcium-Pumping ATPases in Plants H. Sze, Department of Plant Biology}

The regulation of cellular $\mathrm{Ca}$ is one of the most significant functions of plant cells. Changes in cytosolic $\mathrm{Ca}$ is an important intracellular signal, and $\mathrm{Ca}$ in endomembrane compartments is required for the proper functioning of the secretory system. Ca-pumping ATPases terminate the $\mathrm{Ca}$ signal by moving cytosolic $\mathrm{Ca}$ into the extracellular space or into internal stores; however, it is not clear how many distinct Ca pumps exist in plants, and how their activities are regulated. The current goals are to determine the primary structures and functional activities of selected $\mathrm{Ca}$ pumps as a step to understanding their regulation and subcellular location. At least two biochemical types of Ca pumps could be distinguished: a PM-type that bound and was activated by calmodulin, and another (ER-) type that did not bind calmodulin. PM-type pumps were localized on endomembranes and the plasma membrane, while the ER-type was mainly associated with endomembranes. To identify and characterize these pumps individually, two distinct genes encoding Ca-ATPase homologs have been isolated from Arabidopsis. ACA3 could complement yeast mutants defective in two $\mathrm{Ca}$ pumps. Furthermore ACA3p formed a Ca-dependent phosphoenzyme of $116 \mathrm{kD}$ characteristic of P-type Ca pumps. These results demonstrate for the first time that a plant Ca-ATPase gene was functionally active in yeast, and that expression in this heterologous system is a crucial step towards understanding the function and regulation of each Ca pump.

\section{University of Massachusetts}

\section{Amherst, MA 01003}

\section{Cellulose Fermentation by Nitrogen-Fixing Anaerobic Bacteria} S. Leschine, Department of Microbiology

In anaerobic environments rich in decaying plant material, the decomposition of cellulose is brought about by complex communities of interacting microorganisms. We are simulating processes occurring in natural environments by establishing biologically-defined bacterial communities that we use as in vitro systems for quantitative studies of cellulose degradation, particularly under conditions of combined nitrogen deprivation. A second aspect of our research involves investigations of the multicomplex cellulase-xylanase enzyme system which is produced by the mesophilic, $\mathrm{N}_{2}$-fixing, ethanol-producing bacterium, Clostridium papyrosolvens $\mathrm{C7}$. This enzyme system catalyzes the depolymerization of crystalline cellulose and other plant cell wall polysaccharides and consists of at least seven distinct extracellular, high-molecular-weightmultiprotein complexes, each with differentenzymatic and structural properties. We are exploring the possibility that a 125,000-molecular weight glycoprotein, found in all complexes and apparently lacking enzyme activity, functions as a 
scaffolding and/or cellulose-binding protein in the multicomplex cellulase-xylanase system. A DNA fragment homologous to Clostridium thermocellum cipA, which encodes the cellulosome scaffoldin, was cloned from C. papyrosolvens $\mathrm{C} 7$ and currently is being analyzed. In addition, we are examining this enzyme system for activities associated with the degradation of the hemicellulosic portion of biomass. The research will provide fundamental information on the physiology and ecology of cellulose-fermenting, $\mathrm{N}_{2}$-fixing bacteria. Also, it will advance understanding of the enzymology of cellulose hydrolysis by anaerobic bacteria. Information obtained will be valuable for the development of practical applications, such as the bioconversion of plant biomass (e.g., agricultural, forestry, and municipal waste materials) to fuels such as ethanol.

\section{University of Massachusetts}

\section{Amherst, MA 01003}

107. Isolation of Genes Involved in a Novel Auxin Biosynthetic Pathway J. Normanly, Department of Biochemistry and Molecular Biology $\$ 174,653$ (FY 95 funds/2 years)

Towards our long term goal of identifying the enzymes and intermediates involved in the tryptophan-independent auxin biosynthetic pathway, we are employing a TLC-based screen for Arabidopsis genes that synthesize indole-3-acetic acid (IAA) in a tryptophan-independent manner. We have pooled a collection of 100,000 individual yeast colonies transformed with Arabidopsis cDNAs from either of two expression libraries. We are currently screening these pools for their ability to metabolize 14C-labeled indole as a means of isolating a gene involved in the first step of tryptophan-independent IAA biosynthesis. We are also using the pooled transformants in an in vivo selection for indole-metabolizing genes. The indole analogs 5-chloro and 5-methyl indole inhibit growth of yeast at millimolar concentrations. Presumably this is due to incorporation of the indole analog into tryptophan and subsequent deleterious effects upon protein structure. An indole-metabolizing gene that diverts the toxic indole analog away from tryptophan synthesis may in fact be involved in the tryptophan-independent IAA biosynthetic pathway. Yeast transformants that are able to grow in the presence of toxic levels of indole analogs will be tested for indole-metabolizing function. Lastly, we have been examining the role of nitrilases in IAA biosynthesis. Feeding studies with 13C-labeled IAN indicate that one of four Arabidopsis nitrilases (NIT2) could play a role in IAA biosynthesis. We will attempt to determine if the nitrilase is functioning in a tryptophan-dependent or -independent IAA biosynthetic pathway. 


\title{
Michigan State University DOE Plant Research Laboratory East Lansing, MI 48824
}

\author{
108. Molecular Basis of Symbiotic Plant-Microbe Interactions \\ F.J. de Bruijn
}

The induction of nitrogen-fixing root and stem nodules on leguminous plants by soil bacteria belonging to the Rhizobiaceae is a highly evolved, complex process, requiring a fine-tuned interaction between the bacteria and their host. The persistence and competitive ability of the microbes in the soil and the rhizosphere of plants are important factors in early stages of rhizobial infection. In order to increase our understanding of these early stages, we wish to understand the molecular basis of the microbial response to common environmental stresses and plant factors secreted into the rhizosphere, to develop user friendly methods for the detection and classification of soil microbes and to explore the use of specific nutritional mediators to create "biased rhizospheres". Once the infection process has been initiated, distinct sets of plant genes are induced, which are involved in nodule ontogeny and in symbiotic nitrogen fixation. In order to understand the regulatory circuits responsible for symbiotic control of the expression of these loci, we wish to elucidate the molecular basis of the signal transduction pathways responsible for nodule-(cell-)-specific expression of plant genes encoding symbiosis-specific proteins (nodulins). In addition, we are using the model legume plant Lotus japonicus to generate novel symbiotic mutants via chemical mutagenesis and to isolate novel symbiosis-specific genes via differential display of messenger RNA's.

\section{Michigan State University DOE Plant Research Laboratory East Lansing, MI 48824}

\section{Molecular Mechanisms That Regulate the Expression of Genes in Plants P. Green

The steady state level of an mRNA depends both on its rate of synthesis and on its rate of degradation. Rapidly accumulating data indicate that degradation rates of mRNAs in plants and other eukaryotes vary over a wide range and can be differentially regulated. However, relative to transcription, the mechanisms that control mRNA stability are poorly understood. A major goal of our research is to elucidate the mechanisms that target highly unstable mRNAs for degradation in plants because these mechanisms provide plants with a means to make rapid changes in gene expression in response to a variety of stimuli. Our studies have resulted in the identification of specific sequences (e.g., DST elements or AUUUA repeats) that markedly accelerate the decay of reporter transcripts in stably transformed tobacco cells and decrease mRNA accumulation in transgenic plants. Mutagenesis experiments have begun to provide insight about the regions within the DST element that are required for instability function and similar experiments on other instability sequences are underway. These sequences are also being used as tools to develop molecular, genetic, and 
biochemical approaches aimed at identifying components of the degradation machinery and steps in the corresponding mRNA decay pathways. In addition, we have begun to characterize the RNases of Arabidopsis as a first step toward differentiating between the RNases that play a role in mRNA degradation and those with other roles in RNA metabolism.

\section{Michigan State University DOE Plant Research Laboratory East Lansing, MI 48824}

\section{Molecular Biology of Plant-Bacterial Interactions S.Y. He}

This project investigates the molecular basis of signalling during pathogenic interactions between plants and bacteria. In the last year, we have demonstrated that Pseudomonas syringae, a model plant pathogenic bacterium, produces a novel surface appendage named Hrp pilus. We have shown that the Hrp pilus is composed of HrpA protein encoded by a hrp gene which is essential for plant-bacterial interactions. The Hrp pilus is hypothesized to mediate injection of bacterial virulence or avirulence proteins into the plant cell directly or indirectly. In support of this hypothesis, we showed that a bacterial avirulence protein, AvrB of $P$. syringae pv. glycinea, triggers the hypersensitive cell death response when expressed only in the plant cell, but not outside the plant cell or in the bacterial cytoplasm. We have also isolated a number of Arabidopsis thaliana plant cell death ( $p c d$ ) mutants that display spontaneous cell death mimicking the hypersensitive cell death response triggered by plant pathogens. Several cell death suppressor (cds) mutations have been identified that effectively prevent cell death in these pcd mutants. Finally, we have obtained evidence that a human programmed cell death gene, $b c l-2$, triggers cell death associated with disease resistance in Arabidopsis thaliana. Further analysis of the $p c d$ and $c d s$ mutants and $b c l-2$ transgenic Arabidopsis plants would facilitate the understanding of the molecular mechanism underlying plant cell death associated with disease resistance.

\section{Michigan State University DOE Plant Research Laboratory East Lansing, MI 48824}

\section{Chloroplast Biogenesis}

K. Keegstra

We are continuing studies on the biogenesis of plastids. Our current efforts are aimed at understanding the transport of cytoplasmically synthesized precursors into chloroplasts. In particular, we are focusing on identifying and characterizing the components of the transport apparatus responsible for mediating this translocation process. Outer envelope membrane polypeptides of $34-\mathrm{kD}, 75-\mathrm{kD}$ and $86-\mathrm{kD}$ have been identified as putative transport components. More recent efforts have identified an inner membrane polypeptide of $110-k D$ 
as well as ClpC, a stromal protein in the heat shock 100 family of molecular chaperones, as additional components of the transport apparatus. cDNA clones and antibodies for all five proteins are available. Current studies are focused on determining the function of each polypeptide and investigating how these polypeptides interact with each other and with precursor proteins to accomplish protein transport into chloroplasts. Finally, we are employing the antibodies and nucleic acid probes for these putative transport components to investigate their expression in various plant tissues that have different types of plastids.

\section{Michigan State University DOE Plant Research Laboratory East Lansing, MI 48824}

\section{Cell Wall Metabolism \\ K. Keegstra, H. Kende, N.V. Raikhel, and J.D. Walton}

Our goal in this project is to address two questions of cell-wall metabolism. The first is to understand how xyloglucan, the major hemicellulosic polymer in dicots, is synthesized. The second is to understand the role of expansins, proteins that loosen cell walls, during the growth of rice cells. The first part of the first objective is to investigate the fucosyltransferase involved in xyloglucan biosynthesis. Purification of the enzyme is nearing completion. The next step will be to isolate cDNA clones encoding the enzyme and to prepare antibodies against the protein encoded by the cDNA clone. The clones and antibodies will be used as molecular probes to investigate the regulation of this biosynthetic enzyme during growth and development. The second part of the first objective will be to investigate the function of a Golgi-localized $40-\mathrm{kD}$ protein that is postulated to have a role in xyloglucan biosynthesis. CDNA clones for this protein have been isolated and sequenced from Arabidopsis. A combination of molecular genetic and biochemical approaches will be used to investigate its role, if any, in xyloglucan biosynthesis. Finally, we will investigate the involvement of expansins in the growth of rice cells. Expansins are recently discovered cell-wall loosening proteins. We shall study the expression of expansin genes and expansin protein by in situ hybridization and immunocytochemistry, and the fine structure of cell-wall polymer alignment in the presence and absence of expansin.

\section{Michigan State University DOE Plant Research Laboratory East Lansing, MI 48824}

\section{Action and Synthesis of Plant Hormones H. Kende}

The objective of this project is to gain knowledge about the biosynthesis and action of the plant hormone ethylene and about the action of gibberellin (GA) in mediating rapid, submergence- induced elongation of deepwater rice. Our research combines physiological, 
biochemical and molecular approaches. We are elucidating the mechanism of positive and negative feedback regulation of ethylene biosynthesis by determining the expression of genes encoding the two enzymes of this pathway, 1-aminocyclopropane-1-carboxylate (ACC) oxidase and ACC synthase. We are also localizing the expression of these two genes with respect to asymmetric growth, e.g. the formation of the apical hook. We are also investigating the mechanism of catalysis of ACC oxidase and its activation by $\mathrm{CO}_{2}$. Signal transduction of ethylene action is being followed in Arabidopsis, where we have identified a number of ethylene-regulated genes. In deepwater rice, ethylene reduces the level of endogenous abscisic acid (ABA), thereby increasing the sensitivity of the plant to GA. We propose to study the effect of ethylene on ABA metabolism, in particular with respect to the activity of $A B A$ oxidase. Since $G A$ is the immediate growth stimulating hormone in deepwater rice, we are studying GA-regulated cell wall extension, mainly regarding the role of expansins in this process, and GA-promoted cell division in the intercalary meristem (IM), using cDNA probes for genes whose products control the progression of meristematic cells through the cell cycle. Using differential display of mRNA, we are also identifying genes in the IM whose expression is regulated by $\mathrm{GA}$.

\section{Michigan State University DOE Plant Research Laboratory East Lansing, MI 48824}

\section{Photoperiodic Induction and the Floral Stimulus}

H. Kende, L. McIntosh, and J.A.D. Zeevaart

A large body of physiological evidence indicates that flower formation is hormonally controlled. In plants exposed to the appropriate photoperiod for flowering, a floral stimulus or florigen is formed in the leaves from where it is translocated in the phloem to the apical meristem. Results of grafting experiments show that the floral stimulus is exchangeable between different response types and is, therefore, very similar, if not identical, in different species. The chemical nature of this floral stimulus is one of the major unsolved problems in plant biology. We propose to investigate two aspects of photoperiodic control of flowering: the regulation of gene expression in the leaves by the photoperiod, and the nature of the floral stimulus. Genes whose expression is modified by photoperiodic induction will be isolated and characterized. These genes, under the control of an inducible promoter, will be used to transform plants to see whether their expression will cause flowering under the wrong photoperiod. To study the nature of the floral stimulus, phloem exudate will be collected from induced and non-induced plants and analyzed for small molecules, RNA, and peptides. Small molecules whose concentrations increase upon induction will be assayed for flower-inducing activity, whereas the role of macromolecules appearing only in exudate of induced plants will be tested by transformation of a photoperiodic plant. 


\title{
Michigan State University DOE Plant Research Laboratory East Lansing, MI 48824
}

\author{
115. Interaction of Nuclear and Organelle Genomes \\ L. Mclntosh
}

$\$ 230,857$

Our research is focused on the molecular and biochemical mechanisms regulating energy transduction and carbon flow in photosynthesis organisms. Molecular/genetic characterization and modification of key enzymes and individual components of membrane complexes are used to understand the mechanisms and regulation of photosynthesis and respiration. Our studies with plant mitochondria revolve around the alternative pathway of respiration and its integration into stress-involved carbon balance in higher plants. We have characterized the alternative oxidase gene, Aox1, from a number of species including Sauromatum guttatum, tobacco, potato, soybean and Neurospora crassa. We have characterized gene regulation of Aox 1 and investigated the biochemical regulation of the oxidase itself. Many of these experiments give results through use of transgenic tobacco and potato lines, some which overexpress the alternative oxidase and others that repress its expression.

Photosystem I (PSI) is a key, but understudied, thylakoid membrane complex responsible for reduction of NADP+ and production of ATP. We have developed a cyanobacterial system allowing for in vivo insertion of site-directed mutations into the core subunits of PSI: PsaA, PsaB, and PsaC. We have further developed a mechanism to isolate second-site pseudo-revertants to specific PsaA, PsaB and PsaC mutations. An efficient genetic system is now being tested for the isolation of all types of suppressor mutations responsible for these revertants and thus examine the principal subunit:subunit interactions within the core of PSI.

\section{Michigan State University DOE Plant Research Laboratory East Lansing, MI 48824}

\section{Sensory Transduction in Plants}

K.L. Poff

The objective of this project is to understand the mechanisms for the acquisition of environmental information via light reception. In particular, we are studying phototropism in flowering plants. This response is under the control of the blue light photoreceptor pigment system(s) which control(s) numerous directional light responses, and is strongly modulated by phytochrome which typically controls responses based on daylength. We have developed a genetic system with which we can dissect the initial steps in the transduction sequences. Screening procedures have been devised and used to identify mutants of Arabidopsis with altered phototropism. Based on such mutants, a major component of phototropism is phototropic adaptation. Under conditions of long term irradiation, plants adapt to the 
irradiation, such that their responses at the beginning and end of a long irradiation are not equal. An understanding of this process of adaptation is necessary to understand the plant's response to the long term irradiations that are important for the plant under field conditions. Our approach to the study of the pathways for phototropism and phototropic adaptation includes genetic, physiological and biophysical characterization of mutants and ecotypes that differ in their phototropic response. Using such mutants, genes involved in phototropism are isolated and identified. This genetic/biophysical/molecular approach should permit access into the transduction sequence, and eventual understanding at the molecular level of the events from reception to the bending responses.

\section{Michigan State University DOE Plant Research Laboratory East Lansing, MI 48824}

\section{Molecular Mechanisms of Trafficking in the Plant Cell N.V. Raikhel}

Maintenance of separate subcellular compartments in eukaryotic cells depends on the correct sorting and targeting of newly synthesized proteins. Thus, mechanisms must exist in the cell to assure that these proteins are targeted to, and subsequently translocated across, the correct intracellular membranes. We are working with proteins destined for different compartments: vacuoles, cell wall and nucleus. We are interested in understanding the molecular determinants of differential protein compartmentalization and identifying the components of the molecular machinery which carry out the sorting process. We have analyzed and characterized sequences responsible for protein sorting to the vacuole and to the nucleus, and have isolated several putative receptors of the endomembrane system and the nuclear import machinery. We are now elucidating the functional complexity of vesicular trafficking and transport to the nucleus.

\section{Michigan State University DOE Plant Research Laboratory East Lansing, MI 48824}

\section{Biochemical and Molecular Aspects of Plant Pathogenesis J.D. Walton}

The objective of this project is to further our understanding of the biochemical events that are important in the interactions between fungal plant pathogens and their hosts. We are studying examples of pathogen factors that determine basic compatibility (the ability of a fungus to infect any plant) and those that determine specificity (the ability of a fungus to infect a particular plant, i.e, the basis of host-range). As examples of basic compatibility factors, we are studying the extracellular enzymes that degrade the polymers of the plant cell wall. Circumstantial evidence suggests that these enzymes are important for colonization of host 
tissue and also can act as triggers of plant defense responses. We have purified enzymes, including pectinases, xylanases, glucanases, cellulases, and proteases, from the ascomycetous maize pathogen Cochliobolus carbonum, cloned the corresponding genes, and tested their importance in pathogenesis with targeted gene disruption. The host-selective toxin, HC-toxin, is a specificity determinant because it affects only maize of certain genotypes. We established that the maize resistance gene $\mathrm{Hm}$ encodes a reductase that detoxifies HC-toxin, and have now shown that the site of action of HC-toxin is histone deacetylase, a nuclear enzyme that influences chromatin structure and gene expression by modifying core histones. We are studying why inhibition of this enzyme leads to the establishment of a compatible (susceptible) disease interaction. In the fungus, $\mathrm{HC}$-toxin production is controlled by a single genetic locus, TOX2. TOX2 contains multiple copies of multiple genes necessary for HC-toxin synthesis, including a large cyclic peptide synthetase, a fatty acid synthase, and a toxin export pump. All of these genes are present only in isolates of $C$. carbonum that make HC-toxin and are distributed over $540 \mathrm{~kb}$ on the same dispensable chromosome.

\section{Michigan State University DOE Plant Research Laboratory East Lansing, MI 48824}

\section{Developmental Biology of Nitrogen-fixing Cyanobacteria C.P. Wolk}

Anabaena and related filamentous cyanobacteria utilize reductant generated by photosynthesis in vegetative cells to fix nitrogen gas within spaced, differentiated cells called heterocysts. We are elucidating the dependency relationships, and the roles, of genes whose collective function allows Anabaena to fix.nitrogen (an oxygen-sensitive process) in the presence of oxygen. The heterocyst envelope plays a key role by limiting the rate of entry of oxygen into heterocysts. We are studying hep genes, required for synthesis or stabilization of the polysaccharide layer of that envelope. A sequence located between 536 and $707 \mathrm{bp}$ upstream from the transcriptional start site of gene hepA appears to be required for activation of that gene, and the gene upstream from that region appears to repress that activation. A transposon mutation in hepK, whose predicted product is highly similar to histidine protein kinases of two-component regulatory systems, blocks synthesis of envelope polysaccharide but leaves synthesis of heterocyst envelope glycolipid unimpaired. Downstream from hepK is a gene whose product is very similar to DNA helicases. We are trying to determine whether the presumptive helicase gene is also required for normal differentiation. In addition, we have analyzed the hepB region and we have observed that a mutation in $r b P$, a gene involved in synthesis of the lipopolysaccharide at the periphery of vegetative cells, leads to faulty deposition of heterocyst envelope glycolipid. This work will facilitate understanding of cellular differentiation, pattern formation, and biological conversion of solar energy. 


\title{
Michigan State University DOE Plant Research Laboratory East Lansing, MI 48824
}

\author{
120. Frankia Genetics \\ C.P. Wolk, T. Newman, S.Y. He, and F.J. de Bruijn
}

$\$ 173,858$

Frankia spp., bacteria in the Gram positive actinomycete group, form nitrogen fixing nodules with a wide variety of non leguminous woody plants. Frankia nodulated plants have great, world wide potential as sources of fuel, fiber, shade, reforestation and forage. The potential of these plants, and opportunities for understanding the interesting biology of Frankia, are held strongly in check by the current inability of these bacteria to be genetically manipulated. Transfer of DNA to Frankia requires a suitable replicon, a means of transferring the DNA, appropriate selective markers, avoidance of endogenous DNA restriction systems, and a suitable choice of strain. Our short term objective is to develop techniques for stable, reproducible genetic transformation of Frankia. Once gene transfer to Frankia is achieved, we will proceed to develop techniques for transposon mutagenesis of, and gene replacement and use of reporter genes in, this organism. These techniques will then be applied to achieve, as a longer-term objective, a basic understanding of the genetic components involved in the generation of $\mathrm{N}_{2}$ - fixing symbioses of Frankia with higher plants.

\section{Michigan State University DOE Plant Research Laboratory East Lansing, MI 48824}

\section{Environmental Control of Plant Development and its Relation to Plant Hormones \\ J.A.D. Zeevaart \\ $\$ 211,857$}

Plant growth and development are affected by environmental factors such as daylength, temperature, and moisture. Hormones often function as chemical messengers between the perception of an environmental factor and the morphological expression. The objectives are to identify the hormones involved, how their synthesis and catabolism are regulated by the environment, and how they act. In long-day rosette plants, stem growth in long days (LD) is caused by an increase in gibberellin (GA) levels, particularly in the shoot tips. The obvious question is: which step(s) in the GA biosynthetic pathway is (are) stimulated by LDs? As a first step towards answering that question, GA 20-oxidase genes have been cloned from Arabidopsis and spinach and functionally expressed in E. coli. GA 20-oxidase is a multifunctional enzyme that oxidizes and eliminates $C-20$ of the $G A$ skeleton $\left(G A^{53} \rightarrow G A_{44}\right.$ $\rightarrow G A_{19} \rightarrow G A_{20}$ ). In spinach, GA 20-oxidase mRNA is higher in plants in LD than in short days (SD), with highest levels of expression in the shoot tips and elongating stems. This expression pattern of GA 20-oxidase is consistent with the higher GA levels found in spinach plants grown in LD than in SD. 
Abscisic acid (ABA) is a plant hormone whose synthesis is greatly stimulated when plants experience a water deficit. Conversely, $A B A$ is rapidly inactivated to phaseic acid (PA) when plants are rehydrated. The objective of our studies is to find out how the metabolism of ABA is regulated by the water status of the tissue. Two new mutants of Arabidopsis, aba2 and aba3, have been characterized biochemically. The aba2 mutant is blocked in the conversion of xanthoxin to ABA-aldehyde, whereas aba 3 is impaired in the conversion of $A B A$-aldehyde to $A B A$. The genetic lesion in aba3 is not in the apoprotein, but in the molybdenum cofactor that is required by the aldehyde oxidase.

\section{Michigan State University East Lansing, MI 48824-1101}

\section{Xylan-Degrading Enzymes of Cytophaga xylanolytica}

J.A. Breznak, Department of Microbiology

Studies are being done on the cell-associated "xylanase system" of Cytophaga xylanolytica, an anaerobic gliding bacterium widely distributed in freshwater sediments. An $\alpha$-L-arabinofuranosidase (Araf1) has been purified and characterized and was recently used to generate, in rabbits, high-titer specific anti-Araf1 antibody for subcellular localization studies. The amino acid sequence of three internal, trypsin-generated fragments of Araf1 bore significant similarities to portions of an arabinofuranosidase (asdll gene product) from Bacteroides ovatus V975. Such information has also enabled the preparation of oligonucleotide probes that are being used to clone the Araf1-encoding gene from $C$. xylanolytica. [Separately, we have cloned and sequenced a $1.9 \mathrm{~kb}$ gene from $C$. xylanolytica that encodes an arabinofuranosidase other than Araf1, but which is not expressed by $C$. xylanolytica when growing on oat spelt arabinoxylan.] Three major endoxylanase fractions have been partially purified from $C$. xylanolytica by using preparative PAGE. Studies are in progress to determine the extent of synergy between these and Araf1 during hydrolysis of natural arabinoxylans.

\section{Michigan State University East Lansing, MI 48824}
123. The Role of the Bacterial Surface Glycoconjugates in the Rhizobium/Legume Symbiosis
R.I. Hollingsworth, Department of Biochemistry

The interaction between bacteria of the family Rhizobiaeae and legume plants is an old wellestablished one that is responsible for most of the nitrogen fixed on land. This is important and drives the flux of nitrogen through the various biosystems where it is required for amino acid, nucleoside, carbohydrate and lipid biosynthesis and that of a myriad of other 
intermediates. It is still not known how this symbiosis works since several classes of molecules, especially carbohydrates and glycolipids, are known to be involved. In this project, we are focussing on the interrelationship between the various cell surface carbohydrates and related membrane associated carbohydrates and glycolipids that are synthesized by Rhizobium and whose synthesis impacts on the ability of these bacteria to infect their legume plant hosts. We have had great success in characterizing the structural details of the various cell surface and membrane associated carbohydrates and glycoconjugates from both fast growing and slow growing strains. We have been involved in experiments to determine how perturbation of the biosynthesis of any one class of these molecules leads to changes in the synthesis of the others. This will help delineate which molecules are necessary for the successful outcome of the symbiosis. We are especially interested in how the biosynthesis of chitolipooligosaccharide nod factors is tied into other carbohydrate and glycolipid biosynthesis. Another area of study is the question of bacterial surface compatibility whilst inside of the plant. We have discovered that the biosynthesis of bacterial surface glycolipids is closely related to that of plants suggesting the possibility of gene transfer between these systems.

\section{Michigan State University East Lansing, MI 48824-1312}

\section{Control of Triacylglycerol Biosynthesis in Plants \\ J. Ohlrogge, Department of Botany and Plant Pathology}

Triacylglycerol (TAG) is the major form of carbon storage in seeds of many important crops and the oils extracted from these plant seeds represent a $\$ 20$ billion dollar commodity with a wide variety of uses in the food and chemical industries. The overall objective of this research project is to understand how triacylglycerol biosynthesis is controlled in plants. Whereas leaves, roots and other tissues usually contain less than a few percent of their total lipid in the form of TAG, seed lipids frequently contain over 95\% TAG. A metabolic or biochemical explanation for this dramatic difference in lipid composition has not yet been established. In order to begin to provide such an explanation we will examine the relative importance of possible alternative control mechanisms by examining the in vivo pool sizes of key intermediates in the pathway for TAG biosynthesis. In particular, methods will be developed to measure the plastid pools of acetyl-CoA and malonyl-CoA. In addition, the size and fatty acid composition of the acyl-CoA and diacylglycerol pools in plants which produce different fatty acids will be determined. Leaf and seed tissue will be compared to establish correlations between the different lipid metabolism of these tissues and the pools of the key pathway intermediates. The role of diacylglycerol acyltransferase will be evaluated by examining its expression and kinetic properties in seeds and leaves and the specificity of the CDP:choline phosphotransferase will be examined to determine its ability to provide specific partitioning of unusual fatty acids into triacylglycerol and their exclusion from phospholipids. 


\section{Michigan State University East Lansing, MI 48824}

\section{A National Cooperative for Genetic Engineering of Plant Lipids J. Ohlrogge, Department of Botany and Plant Pathology}

$\$ 60,000$

In order to explore the wide range of potential applications of genetic engineering techniques to the production of useful new plant lipids, it will first be necessary to develop a detailed mechanistic understanding of most aspects of plant lipid metabolism and to acquire genes for the key enzymes and structural proteins. Progress toward these general goals will be greatly facilitated by the creation of a National Plant Lipid Cooperative (NPLC). The NPLC will provide an efficient mechanism for facilitating exchange of ideas, information and research materials among all members of the North American community. Exchange of ideas will occur through the development of an electronic newsgroup and sponsorship of participation of young scientists in regular meetings and workshops devoted to plant lipid metabolism. A major objective, in this regard, will be to encourage young scientists to initiate research programs on ignored topics of strategic importance to the whole field by allocating resources to the development of these areas. Exchange of information will be stimulated by the development and distribution of several databases containing all published information about plant lipid metabolism and the chemical composition of lipids from different plant species. Finally, the NPLC will commission the production and distribution of essential research materials which are not commercially available but which are required in order to pursue new avenues of research.

\section{Michigan State University East Lansing, MI 48824-1319}

\section{Structure-Function Relationships of ADP-glucose Pyrophosphorylase: Manipulation of the Plant Gene for Increased Production of Starch in Plants J. Preiss, Department of Biochemistry $\$ 170,000$ (2 years)}

Structure-function relationships of the ADPglucose pyrophosphorylase from higher plants (potato tuber) will be studied. This research entails the use of amino acid residue chemical modifying reagents (e.g., pyridoxal-5-P, phenylglyoxal, 8-azido-ATP and 8-azido-ADPglucose) in experiments designed to determine the nature of involvement and location of the various amino acids at the catalytic and regulatory (allosteric) sites. The cDNA clones of the large and small subunits of the potato ADPglucose pyrophosphorylase have been expressed in $E$. coli. The expression of these genes and prior chemical modification studies on the purified spinach leaf ADPglucose pyrophosphorylase enable us to do site-directed mutagenesis at various regions of the plant enzyme to gain more insight on the nature of the catalytic and effector sites and function of the two different subunits in the higher plant ADPglucose pyrophosphorylase. Attempts will also be made to understand why the higher plant enzyme 
(a heterotetramer, $\alpha 2 \beta 2$ ) requires two different subunits for high activity. Partial proteolysis experiments and molecular modeling programs are being used to predict and determine the secondary structure of the enzyme. Crystallization and X-ray diffraction studies are being attempted to obtain insight into its three dimensional structure. "Mutant", active plant enzymes that may either be less sensitive to allosteric inhibition by phosphate or that may not require the allosteric activator, 3-phosphoglycerate, for activity will be constructed. These could in the future be used to produce transgenic plants having increased amounts of starch.

\section{Michigan State University East Lansing, MI 48824-1101}

\section{Physiology and Molecular Biology of Lignin-Modifying Enzymes C.A. Reddy, Department of Microbiology}

Research on biodegradation of lignin, a plant polymer that is the second most abundant renewable source of organic carbon in the biosphere, has important applications in biopulping and biobleaching, decolorization and detoxification of pulp and paper mill effluents, and bioremediation of toxic recalcitrant environmental pollutants. Laccases, lignin peroxidases (LIPs), and manganese-dependent peroxidases (MNPs) are three classes of lignin-modifying enzymes (LMEs) that are believed to be important in lignin-degradation by white-rot fungi. Considerable progress has been made on the physiology and molecular biology of LIPs and MNPs and research has intensified in obtaining a better understanding of the laccases. We recently initiated research to study the distribution of LMEs in soil basidiomycetes. These are known to play a dominant role in lignocellulose degradation in soils but practically nothing is known about the enzymology of this process. Our studies indicate that laccases are the dominant LMEs in soil basidiomycetes. Detailed characterization of the laccase from one of these strains including cloning and characterization of the gene(s) encoding this enzyme will be an important focus of research. Furthermore, we will study the laccase of Ganoderma lucidum, an important lignin-degrading white-rot fungus, to obtain a better understanding of the comparative biology of fungal laccases. We will also continue our ongoing studies on characterizing lip and mnp gene expression in wood-grown cultures of Phanerochaete chrysosporium, the most widely studied white-rot fungus, to obtain a better ecological understanding of the regulation of expression of these key LMEs. 


\section{Michigan State University \\ East Lansing, MI 48824-1319}

128. Molecular Physiology of Succinic Acid-Based Fermentations in Anaerobes: Control of Chemical Yield by $\mathrm{CO}_{2}$ Fixation and Electron Donors J.G. Zeikus, Department of Biochemistry $\$ \$ 110,000$

This project will let us understand the fundamental mechanisms regulating the carbon and electron flows in chemosynthetic anaerobes that couple $\mathrm{CO}_{2}$ fixation to succinate production. Anaerobiospirillum succiniciproducens, the best characterized high succinate producer, will be used as a model to study the key function of phosphoenolpyruvate (PEP) carboxykinase in succinate production. Unlike most PEP carboxykinases which catalyze oxaloacetate decarboxylation, $A$. succiniciproducens PEP carboxykinase catalyzes PEP carboxylation. This enzyme's structural determinants for substrate affinity and the genetic regulation of its expression will be studied to identify the mechanism(s) determining its $\mathrm{CO}_{2}$-fixing activity. A newly discovered Actinobacillus species that produces up to three times more succinate than A. succiniciproducens will be used for metabolic and fermentative studies. These studies will include the determination of Actinobacillus carbohydrate metabolism as well as the effect varying medium parameters (i.e., $\mathrm{pCO}_{2}$ and electron donors) have on this organism's carbon and electron flows. The effect of electricity-derived electrons on bacterial growth and fermentation balance will be studied, using anaerobic strains engineered to overexpress various enzymes (e.g., PEP carboxykinase, formate dehydrogenase, hydrogenase) as model succinate fermentation systems. 5-aminolevulinate, a direct product of succinate, is a useful bioactive compound. Genetic reguiation of 5-aminolevulinate synthase expression and medium parameters will be studied to optimize 5-aminolevulinate synthesis from succinate by Actinobacillus and recombinant $E$. coli strains. The metabolic balance and the limiting step(s) of this fermentation will be determined, helping define engineering strategies to enhance the metabolic flux toward aminolevulinate production.

\section{University of Michigan Ann Arbor, Ml 48109-1048}

\section{CLV Signaling in Meristem Development}

S. Clark, Department of Biology

The shoot meristem of higher plants is responsible for initiating all above-ground organs. Thus the genes regulating shoot meristem function are required for cell proliferation and differentiation, and for the proper number, spacing and size of primordia such as leaves and flowers. To function as a site of continuous organ formation, the shoot meristem must be able to carry out two fundamental processes. The first function is the maintenance of a population of undifferentiated cells at the center of the meristem. As this population divides, it provides new cells for incorporation into organ primordia. The second process is to direct 
progeny of the undifferentiated cells towards organ formation and eventual differentiation. This transition from an undifferentiated to differentiated state occurs at the flanks of the shoot meristem, and is regulated in strict balance with the proliferation of the undifferentiated cells

We have found that the CLVIoci (CLV1, CLV2 and CLV3) in Arabidopsis regulate the balance between proliferation and differentiation. clv mutants accumulate over 1000-fold more undifferentiated cells than wild-type plants. CLV1 encodes a receptor-like kinase, and may relay positional information through a signal transduction cascade. Our work focuses on identifying other members of the CLV1 signaling cascade through both genetic and biochemical approaches.

\section{University of Minnesota Minneapolis, MN 55455}

\section{Genetics of Bacteria that Utilize One-Carbon Compounds R.S. Hanson, Department of Microbiology}

Methylotrophic bacteria are bacteria that grow on one carbon compounds including methane, methanol, methylamines and some halomethanes. Some methylotrophs also grow on multicarbon compounds including sugars and induce the synthesis of enzymes involved in metabolism of one carbon compounds only in their presence. One enzyme, methanol dehydrogenase (MDH) encoded by mxaF, can comprise $20 \%$ of the cellular protein when induced. The expression of this enzyme and other proteins in the same regulon involves very complex regulatory circuits that include at least three sets of two-component regulatory systems. The sequences of two sets of the regulatory genes, $m \times c Q: m \times c E$ and $m \times c \mathrm{M}: m \times c D$ have been determined. The protein encoded by $m \times c \mathrm{E}$, a DNA binding regulatory protein that is required for synthesis of $\mathrm{MDH}$, has been partially purified and its binding site upstream of $m \times a F$ has been identified. We are attempting to characterize proteins encoded by other regulatory genes.

Synthesis of one enzyme required for methane metabolism, a soluble methane monooxygenase, is repressed by copperions. Cells grown in the presence of one micromolar copper or higher lack this enzyme and contain a different particulate methane monooxygenase. We are using mutant analysis and characterization of copper binding proteins that may have a regulatory role to study the control of the synthesis of the soluble methane monooxygenase. 


\section{University of Minnesota \\ Minneapolis, MN 55455-0312}

131. The Mechanism of Switching from an Acidogenic to a Butanol-Acetone Fermentation by Clostridium acetobutylicum

P. Rogers, Department of Microbiology

$\$ 100,000$

The overall objective of this project is to elucidate the detailed mechanism by which solventforming bacteria such as Clostridium acetobutylicum regulate the well known shift in fermentation pathway between alcohol-acetone and organic acid production. We eventually want to isolate and describe: (1) the regulatory genes and protein elements that determine induction of synthesis of the solvent-pathway enzymes; and (2) how this regulation system interacts with the sporulation and exopolysaccharide formation in clostridia. Thus we are searching for regulatory elements that may involve environmental sensors and also DNA binding proteins that act as enhancers or positive control proteins.

We have produced 40 pleiotrophic Tn 916 transposon-induced mutants of $C$. acetobutylicum that fail to switch to solvent formation, do not form granulose bodies and do not sporulate. We are studying complementation of these mutants with a library of $1.0-3.5 \mathrm{~Kb}$ DNA fragments from the parental ATTC824 strain carried on an E. coli/C. acetobutylicum shuttle vector, pKR13, produced in our laboratory. We are examining clones that revert Tn 916 mutants to granulose-positive $\left(\mathrm{Gra}^{+}\right)$. Sequencing of these clones is underway. $C$. acetobutylicum 824 carries a $210 \mathrm{~Kb}$ plasmid, pSOL1, which carries the solvent genes, aad, $c t f A, c t f B$, adc (T. Popoutsakis). Using pulse-field gel electrophoresis together with a $4.4 \mathrm{~kb}$ prob DNA containing the solvent genes we are surveying the Tn 916 mutants for loss or alteration of the pSOL plasmid. Recently, Wilkinson, S.R., (FEMS Microbiol. Rev. 17:275, 1995) reported that the onset of solvent formation by C. biejerinckii is modulated by SpoOA. Using a specific SpoOA-mRNA prob we are examining expression of SpoOA in our Tn916 mutants.

\section{University of Minnesota}

\section{St. Paul, MN 55108-1095}

132. Isolation and Characterization of Ammonia Monoxygenase of Nitrosomonas

A.B. Hooper, Department of Genetics and Cell Biology

$\$ 101,000$

Oxidation of ammonia $\left(\mathrm{NH}_{3}\right)$ provides energy for growth of this autotrophic bacterium. Ammonia monoxygenase (AMO) catalyzes $\mathrm{NH}_{3}+2 \mathrm{e}^{-}+2 \mathrm{H}^{+}+\mathrm{O}_{2}-\rightarrow \mathrm{NH}_{2} \mathrm{OH}+\mathrm{H}_{2} \mathrm{O}$. Alternate substrates include $\mathrm{CO}$, methane, and halogenated aliphatic and aromatic compounds. Electrons are passed to AMO by a series of carriers from hydroxylamine oxidoreductase and its electron acceptor, cytochrome c-554. The intention is to analyze the structure and mode of action of AMO. Acetylenic substrates derivatize a subunit of AMO allowing us to isolate 
two putative subunits of the enzyme (AMO-A and $-B$ ) and show that they contain iron and copper. Perturbation of the EPR spectrum of membranes with NO or nitrapyrin supports a catalytic role for iron. We have cloned and sequenced genes for the two proteins and generated a model of membrane orientation and possible metal binding sites.

We continue efforts to improve the assay and means of stabilization of an enzyme system which may include proteins in the electron donor pathway and AMO-reductase and AMO-hydroxylase components. The structure of the active site and electron-transfer redox centers will be analyzed by optical, EPR, Mossbauer and other types of spectroscopy. We will test the model of membrane topology by protein chemistry. We will determine the sequence of binding of dioxygen, substrate and addition of electrons and the form of activated oxygen generated by the enzyme. We have determined that the reaction proceeds by a radical rebound using specifically deuterated ethylbenzene substrates.

\section{University of Missouri Columbia, MO 65211}

\section{Cellulose Synthesis and Morphogenesis T.I. Baskin, Division of Biological Sciences}

The goal of this research is to understand plant morphogenesis. The project studies the relationship between morphogenesis and alignment of cellulose microfibrils. Experiments have used roots of Zea mays, because we have a treatment, water stress, that alters root morphology. We have measured the spatial distribution of expansion, in length and in radius, and found differences in the magnitude of radial expansion at different positions. The alignment of cellulose microfibrils has been quantified with electron microscopy of metal replicas of the inner-most wall layer. The microfibrils are transverse to the long axis of the root throughout much of the growth zone; however, comparing locations having different magnitudes of radial expansion, there is no detectable difference in cellulose alignment. Additionally, the alignment of cellulose microfibrils has been compared to that of cortical microtubules. In regions with transverse cellulose, the two elements are co-aligned, but when the alignment of cellulose becomes oblique, forming a helix around the cell, both left and right handed helices are formed, whereas microtubule helices are uniformly of a single handedness. The composition of the cell wall in the regions where radial expansion is chiefly regulated is being studied non-invasively with Fourier-transform, infrared microspectroscopy. The work will deepen understanding of how microfibrils shape the cells and tissues of higher plants. This research will uncover basic mechanisms of plant morphogenesis and can thus be expected to have a positive impact on applied efforts to optimize plant growth for human benefit. 


\section{University of Missouri Columbia, MO 65211}

\section{Dosage Analysis of Gene Expression in Maize J. Birchler, Division of Biological Sciences $\$ 202,000$ (FY 95 funds/2 years)}

The goal of this project is to understand further the basis of dosage sensitive regulatory effects on gene expression in maize. These effects act directly or inversely on the quantitative level of gene products when the dosage of specific chromosomal segments is varied. It has long been known that addition or subtraction of chromosomal segments relative to the normal diploid has detrimental effects on the vigor of plants. The experiments proposed seek to understand the molecular basis of these aneuploid syndromes as well as to understand the mechanisms of gene expression that are involved. One set of experiments is to test for a correlation between small regions of chromosome arm $10 \mathrm{~L}$ that produce inverse and direct dosage effects on selected monitored RNAs and that produce aneuploid effects on kernel size. This chromosome arm will be divided into several segments--some with known kernel size effects. These smaller regions will be tested for a correlation with the molecular dosage effects that are found in the larger segment. Also, conditions that make the aneuploid effects more extreme will be tested as to whether the dosage effects are also of greater magnitude. To determine whether the effects can be reduced to the action of single genes, two projects are proposed. First, dosage series including three known regulatory genes ( $V p 1, C 1$ and $R 1$ ) effective on the anthocyanin pathway are being examined for effects on the RNAs for the biosynthetic enzymes. One of these, the C1 locus, is known to give a dosage effect and might well be a single gene example of a direct regulator. Any effects found with the whole arm dosage series will be tested whether they are due to the respective regulatory gene by recombining a loss-of-function allele in each case onto the $B-A$ translocation used to generate the series. If the regulatory gene is responsible for the whole arm effect, then the recombined translocation will no longer be effective. Secondly, modifiers will be induced that increase or decrease the amount of pigment present in kernels that carry a single functional copy of $\mathrm{C} 2$ in the endosperm using the Mutator transposon system. Modulations of this gene encoding chalcone synthase can be recognized phenotypically. Heritable modifiers will be tested for a dosage effect on C2 RNA. Lastly, run-on transcription assays will be conducted on some of the most extreme inverse and direct effects on Alcohol dehydrogenase-1 to test whether the rate of transcription is being modulated by these changes in chromosomal dosage. These experiments will provide information on the mechanisms of gene expression involved with these dosage effects and the relationship to the altered vigor of plants that have changes in chromosome number. 


\title{
University of Missouri Columbia, MO 65211
}

\author{
135. Position Effect as a Determinant of Variegated Pigmentation in Maize \\ K.C. Cone, Division of Biological Sciences \\ $\$ 131,500$
}

The aim of this project is to understand the mechanisms that control epigenetic variation in gene expression. We are studying $\mathrm{Pl}-\mathrm{Bh}$, a gene that controls the synthesis of purple anthocyanin pigments in the maize plant. Plants carrying $P I-B h$ exhibit an unusual pattern of pigmentation characterized by variegation, rather than the usual uniform pattern of pigmentation. Because the nucleotide sequence of $\mathrm{Pl}-\mathrm{Bh}$ is nearly identical to the normal $\mathrm{PI}$ allele, our hypothesis is that the variegated pattern of $\mathrm{Pl}-\mathrm{Bh}$ expression is due to an epigenetic position effect. To address this idea, we are taking molecular and genetic approaches. By treating nuclei with DNasel, we showed that $P I-B h$ DNA is less susceptible to nuclease digestion than PI DNA is. This suggests a difference in chromatin structure between the two alleles. To ask what cis-acting sequence might be controlling this difference, we tried to uncouple $P I-B h$ from its cis-linked regulatory elements by recombination. In a screen of 50,000 plants, we did not recover any non-variegated progeny. This result suggests that the genetic determinants of the Pl-Bh phenotype are very tightly linked to the gene. Finally, to ask if the pattern of $P I-B h$ methylation spreads from the Pl locus to adjacent genes, we assayed the methylation status of RFLP markers surrounding the gene; none are differentially methylated in lines carrying $P I-B h$ relative to lines with $P I$. Thus, any cis-linked regulatory element controlling methylation must not exert its effect over a very long range.

\section{University of Missouri Columbia, MO 65211}

\section{Molecular Analyses of Nuclear-Cytoplasmic Interactions in the Genus Zea K.J. Newton, Division of Biological Sciences}

We are exploiting evolutionary divergence to identify cooperating nuclear and mitochondrial elements involved in mitochondrial gene expression. Cytoplasmic genomes from three teosintes have been introgressed into different maize nuclear backgrounds. The major objective of the current project is to use the maize nuclear/teosinte cytoplasmic combinations to identify regulators of mitochondrial transcription. We have discovered an unusual mitochondrial promoter of a mitochondrial cytochrome oxidase gene ( $Z p-\operatorname{cox} 2)$ which requires a specific dominant allele of a nuclear gene $(M c t)$ to be active. We are testing whether the same nuclear factor is responsible for the quantitative differences we have observed in transcripts for the two other mitochondrial cytochrome oxidase genes and whether the quantitative differences reflect differences in transcription initiation. The results from this study should indicate if there is a multiplicity of transcription factors required for plant 
mitochondrial gene activity. We will also investigate whether we can use this system to detect additional transcription factors -- influencing genes that code for other mitochondrial components. We are especially interested in identifying any factors that might be involved in the coordinate regulation of mitochondrial gene expression. Other goals include investigating the possible developmental regulation of the unusual $Z p-c o \times 2$ promoter by $M c t$, as well as identifying the source of the $Z p-c 0 \times 2$ promoter.

\section{University of Missouri Columbia, MO 65211}

\section{Targeting and Processing of the Thiol Protease Aleurain} J.C. Rogers, Biochemistry Department

We study a plant vacuolar thiol protease, aleurain, that is structurally and functionally equivalent to a mammalian lysosomal protease, cathepsin $\mathrm{H}$. These unique enzymes are poor general endoproteases but have active aminopeptidase activity; they undergo unusual processing such that a short minichain is cut from the enzyme prosequence and disulfide-linked to an extra cysteine in the mature large chain. Aleurain is expressed in most cells in barley but its functional importance to the plant is unknown. We have shown that aleurain is a marker for an acidified, lytic vacuolar compartment distinct from the protein storage vacuole compartment in plant cells that contain storage proteins. As the vacuolar compartment increases in size, the two separate types of vacuoles appear to merge; this may be a mechanism by which storage proteins are exposed to proteases to initiate their degradation. An understanding of mechanisms regulating targeting of aleurain and of storage proteins to their separate vacuoles, and mechanisms by which the two compartments may merge, may provide new approaches towards more effective mobilization of protein reserves. An understanding of mechanisms that control the activation of protease proenzymes may enhance our ability to protect or mobilize those reserves at specific times during plant development. We have identified and cloned a potential receptor protein that binds proaleurain and directs it into the clathrin-coated vesicle pathway to the acidic, lytic vacuole. Future work will expand an understanding of that pathway by identifying and characterizing other soluble vacuolar proteins that are bound by the same receptor. Cytoplasmic proteins that interact with the cytoplasmic tail of the receptor, and thereby participate in the process by which the receptor with its ligand is segregated into vesicles for transport to the vacuole, will be identified and characterized. Another marker for the specific acidic, lytic vacuolar compartment will be obtained by purifying the protease that specifically "clips" proaleurain upon its entry into that compartment. The functional importance of aleurain will be investigated by constructing mutant Arabidopsis plants that do not express the Arabidopsis equivalent of that enzyme. 


\section{University of Missouri Columbia, MO 65211}

\section{Genetics of the Sulfate-Reducing Bacteria}

Sulfate-reducing bacteria obtain energy from anaerobic respiratory pathways and various fermentations that proscribe the environments in which they can flourish. The electron transport pathways used for these energy generating processes are poorly delineated. We are developing genetic tools to complement the biochemical approaches used to understand these processes. We have created a random mutagenesis tool for Desulfovibrio from transposon $\mathrm{Tn} 7$ that carries a kanamycin resistance cassette. We have shown that elevating the concentration of a protein necessary for the random transposition, TnsE, increases the frequency of transposition, however, cells carrying the cloned tnsE are impaired for growth relative to the wild type. The gene for cytochrome $c 3$ has been cloned, sequenced, and the putative promoter region identified. Confirmation of the function of this region is an immediate goal. A strong promoter for Desulfovibrio has been identified from a promoter probe library. The transcriptional start site has been identified by primer extension and the region of the promoter shows no recognizable homology with known Escherichia coli promoters.

\section{Mount Sinai School of Medicine New York, NY 10029}

\section{The Respiratory Chain of Alkaliphilic Bacteria}

The respiratory chain complexes of extremely alkaliphilic Bacillus species are present in very high concentrations and resemble the general organization of the mitochondrial respiratory

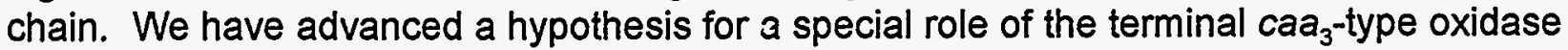
in forming a complex with the ATP synthase of the alkaliphile during oxidative phosphorylation at $\mathrm{pH}$ values above 9.5. Current work on the respiratory chain is therefore most intensely focused on this complex and on the cytochrome $d$ that might be expected to complement this oxidase in conventional organisms. A deletion strain, in which the cta operon encoding the $\mathrm{caa}_{3}$-oxidase is disrupted is indeed found to have elevated levels of cytochrome d. However, the deletion strain is nonetheless unable to grow on non-fermentative carbon sources at either near neutral pH or $\mathrm{pH}$ 10.5. The studies this year will include attempts to further overexpress the cytochrome $d$ complex to see whether complementation is then observed. A full copy of the cta operon is being constructed in a vector that will allow studies of specific mutants and their capacity to restore growth on malate in the deletion strain. 


\section{National Renewable Energy Laboratory Golden, CO 80401}

\section{The Water-Splitting Apparatus of Photosynthesis $M$. Seibert, Center for Basic Sciences}

The focus of our research has been on understanding structural and functional aspects of the reaction center (RC) and the water-oxidizing system of photosystem II (PSII). Langmuir and low angle $\mathrm{X}$-ray diffraction techniques were used to determine the cross-sectional surface area (in the membrane plane, $26 \mathrm{~nm}^{2}$ ) and the length (perpendicular to the membrane, 4.3 $\mathrm{nm}$ ) of the isolated PSII D1-D2 RC complex. Despite analogies with the bacterial RC, the PSIl complex is considerably shorter in length and is, geometrically, a much more regular solid structure. These PSII RC dimensions as well as our dimensions for the in situ PSII core complex obtained last year using Scanning Tunneling Microscopy overlay easily onto recent electron density maps of isolated PSII core complexes reported from other laboratories. Our data support the idea that the active PSII core is a dimeric structure. The pigment stoichiometry of the PSII RC was determined to be 4 core chlorophylls per 2 pheophytins (analogous to the bacterial case), but at least two additional peripheral chlorophyll are also commonly bound and residual CP47 contamination of RC preparations is common. Work identifying high-affinity PSII Mn ligands associated with $\mathrm{O}_{2}$ evolution focused on correlating steady-state and flash studies. One of four bound $\mathrm{Mn}$ detected previously by steady-state measurements is photooxidized by $\mathrm{Y}_{z}^{+}$and is bound to a carboxyl residue. Chemical modification of PSII membranes with a carboxyl-modifier unimasks a new low-affinity site that also binds a photooxidizable $\mathrm{Mn}$, and the ligand to that $\mathrm{Mn}$ is not removed by histidine chemical modification.

\section{University of Nebraska Lincoln, NE 68588-0118}
141. Tomato Bushy Stunt Virus and DI RNAs as a Model for Studying Mechanisms of RNA Virus Replication, Pathogenicity and Recombination
T.J. Morris, School of Biological Sciences, and A.O. Jackson, University of California, Berkeley
$\$ 129,992$

Our research on tomato bushy stunt virus (TBSV) has focused on the characterization of determinants important in replication, pathogenicity and recombination. TBSV is a small RNA virus encoding five genes. It has a broad dicot host range and causes serious diseases of plants. Different strains vary in virulence by generating linear deletion mutants called defective interfering RNAs (DI RNAs) which compete with parental virus and reduce the severity of disease symptoms. To understand the attenuation process, we have been systematically analyzing plant and protoplast infections initiated from in vitro generated 
transcripts of clones of several Tombusviruses and derived DI RNAs. We have made progress in the following areas by studying infections of mutant viral genomes, chimeric viral genomes and mutant DI RNAs: 1) The molecular characterization of cis-elements important in replication of the DI RNAs and preliminary analysis of cis elements important in virus assembly; 2) The development of a model to explain the de novo generation and evolution of DI RNAs with a focus on understanding the basic mechanisms of the RNA recombination process; 3) An evaluation of the function of each of the viral genes important in the virus infection process; and 4) An evaluation of plants engineered to express DIs transgenically for enhanced disease resistance.

\section{University of Nebraska Lincoln, NE 68583}

\section{Enzymology of Aceticlastic Methanogenesis} S.W. Ragsdale, Department of Biochemistry

$\$ 102,000$

Methanogenic microorganisms are responsible for making all the natural gas present on earth. Acetic acid is the major source of methane. Most of the proteins involved in this process have been previously identified and their cofactors have been characterized by a number of laboratories. We want to determine how these enzymes function and have initiated mechanistic studies of key enzymes involved in fermenting acetic acid to methane. We are using a variety of spectroscopic, electrochemical, and kinetic methods to identify the intermediates in the pathway of methane formation and determine which steps in the pathway control the overall rate of methane synthesis. We are focusing on intermediates formed from the cleavage of the $C-C$ and $C-S$ bonds of acetyl-CoA by two key enzymes in the pathway, CO dehydrogenase/acetyl-CoA synthase (CODH/ACS) and a corrinoid/iron-sulfur protein. After acetyl-CoA is disassembled to its component methyl, carbonyl, and CoA adducts, the bound carbonyl group is oxidized to carbon dioxide. $\mathrm{CO}$ oxidation is also catalyzed by the $\mathrm{CODH}$ from photosynthetic organisms. This $\mathrm{CODH}$ will be studied as a model for the $\mathrm{CO}$ oxidation site of the methanogenic and acetogenic enzymes. The final steps of methanogenesis include the reduction of methyl-coenzyme $M$ to methane by methyl-CoM reductase (MCR). This step generates a disulfide product that requires re-reduction by a heterodisulfide reductase (HDR). We are performing mechanistic studies of the MCR and HDR. These studies are hoped to contribute significantly to our understanding of how nature makes methane. 


\section{New York University New York, NY 10003}

\section{Asparagine Synthetase Gene Regulation and Plant Nitrogen Metabolism G. Coruzzi, Department of Biology

We are studying asparagine metabolism in plants using molecular genetic approaches in Arabidopsis thaliana. Inorganic nitrogen assimilated initially as glutamine is converted into asparagine, an inert amino acid that serves to store/transport assimilated nitrogen. Studies on the genes involved in asparagine biosynthesis may therefore have significance to improving nitrogen use efficiency in plants. HPLC analysis has shown that asparagine, which has a high nitrogen:carbon ratio, is preferentially synthesized under conditions of carbon limitation (in the dark) or under nitrogen excess. We have shown that the transcription of the asparagine synthetase ASN1 gene is expressed at high levels in the dark and is dramatically repressed by either light or sucrose. Sucrose repression of ASN1 expression is reversed by the addition of an organic nitrogen source. We propose that plants have a "metabolite-sensing" mechanism whereby ASN1 transcription is activated when the ratio of organic nitrogen is high relative to carbon. This serves to convert anabolically "reactive" glutamine into inert asparagine for storage under conditions of nitrogen excess or carbon limitation. We have defined cis-elements and trans-acting factors involved in this light/sucrose activated transcriptional repression of AS gene expression. Other members of the gene family, ASN2 and ASN3, genes show distinct patterns of gene regulation compared to ASN1. We have developed a positive genetic selection to isolate Arabidopsis mutants impaired in the light/sucrose regulation of AS1 gene expression. The phenotypic analysis of these mutants may define how the regulation of asparagine biosynthesis affects plant growth and nitrogen use.

\section{North Carolina State University Raleigh, NC 27695-7905}

\section{Bioenergetic and Physiological Studies of Hyperthermophilic Archaea R.M. Kelly, Department of Chemical Engineering

The objectives of this project are to examine the biochemical characteristics of several hydrolytic enzymes produced by heterotrophic, hyperthermophilic archaea and relate the function of these enzymes to particular physiological and bioenergetic roles. The study focuses on two members of the Thermococcales, Thermococcus litoralis (Topt $88^{\circ} \mathrm{C}$ ) and Pyrococcus furiosus (Topt $98^{\circ} \mathrm{C}$ ). Both are obligately anaerobic heterotrophs which grow in the presence or absence of reducible sulfur compounds. A novel, intracellular protease (Pfpl) has been isolated from $P$. furiosus which was shown to exist in vitro in several functional homomultimeric forms including a trimer, a hexamer and an assembly with a molecular mass in excess of $200 \mathrm{kDa}$. The gene for this protease, which was isolated, sequenced and 
expressed in active form in Escherichia coli, corresponded to a polypeptide of $18.8 \mathrm{kDa}$ and was found to be homologous to open reading frames of yet unidentified function in several prokaryotic and eukaryotic genomes, including $E$. coli and Methanococcus janaschii. The physiological function of this protease is being examined, especially in relation to an archaeal proteasome which was isolated from $P$. furiosus cell extracts and characterized biochemically. T. litoralis was shown to produce an extracellular polysaccharide in continuous culture which was composed mainly of mannan and which was involved in biofilm formation. Bioenergetic issues related to the facultative reduction of sulfur by this organism are being studied in relation to polysaccharide formation. Several glycosyl hydrolases and proteases from these organisms have been identified and are being investigated for their biochemical and physiological features.

\section{North Carolina State University Raleigh, NC 27695-8008}

\section{Transcription Factors in Xylem Development \\ R. Sederoff, M. Campbell, R. Whetten, and D. O'Malley, Department of Forestry $\$ 106,633$}

Xylem is a specialized tissue found in land plants. It is critical for both mechanical support and water and solute transport in plants. Trees accumulate massive quantities of xylem and, in doing so, form wood. Therefore, xylem is not only important in plant growth and development but is also a major source of renewable biomass. Despite its importance, little is known about the regulation of xylem development. We hypothesize that xylem development is regulated, at least in part, by transcription factors. Transcription factors play key roles in the regulation of plant and animal development. For example, members of the Myb class of transcription factors have been found to regulate developmental processes in a variety of organisms, including blood formation in animals and pigmentation patterns in plants. Recently, genes encoding Myb homologues were cloned from the developing xylem of loblolly pine. These myb genes appear to be much more abundantly expressed in developing xylem than in other tissues. These results are consistent with the hypothesis that these genes encode Myb homologues which regulate transcriptional events during xylogenesis. During the course of this work, this hypothesis will be tested using a number of experimental approaches designed to clarify the function and regulation of the pine xylem Myb homologues. These experiments should therefore result in a greater understanding of the regulation of a fundamental process in plant development. Additionally, these experiments should suggest strategies for the genetic engineering of wood. 


\section{University of North Carolina Chapel Hill, NC 27599-3280}

\section{Novel Control of Signal Delivery from the Pseudomonas syringae avrRPM1 Gene to Arabidopsis thaliana \\ J.L. Dangl, Department of Biology \\ $\$ 102,000$}

We are interested in determining molecularly how plants sense and respond to pathogens. Recently, we and others have cloned several of the plant genes controlling recognition of pathogens. Products of these disease resistance genes recognize the product of a particular pathogen avirulence gene, and this recognition leads to disease resistance. If the plant lacks the correct resistance gene function, or if the pathogen lacks the corresponding avirulence gene, disease results. We use Arabidopsis as a model plant and a bacterial pathogen, Pseudomonas syringae, which can cause leaf spot disease on a variety of crop plants. Our DOE funded research concerns specifically how the avirulence gene signal triggers disease resistance. We have shown that the avirulence-encoded protein can interact with its corresponding disease resistance gene physically in yeast, and we are using a variety of approaches to understand this interaction. Oddly, in a particular $P$. syringae strain the avirulence gene in question is rapidly rendered functionally irrelevant after inoculation of resistant plants, but not susceptible plants. Thus, this strain of pathogen can alter expression of the avirulence gene in a manner dependent on which plant it infects. We are currently studying the mechanism of this avirulence gene inactivation. A detailed understanding of the molecular events leading to plant disease resistance is required for design of engineered crop plants with enhanced disease resistance.

\section{University of North Carolina Chapel Hill, NC 27599-3280}

\section{Map Based Cloning of the Enhancer of Gene Silencing 1 Locus Which Enhances the Silencing of a Foreign Gene in Arabidopsis S.R. Grant, Department of Biology

The ability to introduce foreign genes into plants has changed the way that agricultural technologists can approach crop improvement. However in many cases, genes introduced into plants do not behave as we would expect due to gene silencing. Gene silencing inhibits expression of some foreign genes and simultaneously inhibits expression of plant genes with strong structural similarity to an introduced gene. It is unpredictable occurring spontaneously in late stages of development or appearing after two genetically manipulated plants are mated. The mechanism that leads to gene silencing is completely unknown. It does not cause permanent mutation of the affected genes since they can become reactivated. Silencing may be caused by a system of gene regulation that scientists could only begin to see working when they started to introduce genes into organisms. We have identified 
mutations in two Arabidopsis genes that affect the mechanism of gene silencing. These mutations enhance the silencing of a highly expressed foreign gene so that it occurs in embryonic tissues in all plants. The goal of this project is to clone one of these genes called enhancer of gene silencing 1 in order to better understand the mechanism of gene silencing. Taking advantage of the powerful Arabidopsis genome database and the materials collected by the Arabidopis Stock Center in Ohio, we have mapped the gene to a small interval (4 centimorgans) on the first chromosome. The Stock Center provided us with large Arabidopsis DNA clones that overlap to cover the interval. During the second year of the project, we will use sequences from the overlapping clones to reduce the interval in which the gene must be to a region small enough to introduce into plants. The egs $1 \mathrm{gene}$ will finally be identified by its ability to restore the expression of the silenced gene when introduced in the mutant.

\section{Ohio State University Columbus, OH 43210-1292}

\section{Control of Sugar Transport and Metabolism in Zymomonas mobilis $T$. Conway, Department of Microbiology

Glucose transport in Zymomonas mobilis is via facilitated diffusion and the corresponding gene, glf, is co-transcribed with the genes encoding the first three intracellular steps of glucose metabolism. Regulation of the glf-zwf-edd-glk operon is intriguingly complex. Current work is designed to elucidate facilitator expression, function, and communication with the glycolytic enzymes. Fusions of the glf promoter to lacZ are being used to discern the architecture of this efficient promoter and have implicated a region that appears to be involved in increasing transcription in the presence of fructose. Kinetic studies of the facilitator prove that the gif gene product is indeed a facilitator and that it can function properly in recombinant E. coli. Experiments indicate that Glf does not interact with glucokinase to regulate sugar transport. The facilitator exhibits low affinity, high velocity transport properties, with an affinity for glucose that is orders of magnitude greater than for fructose. Catabolite choice at the level of solute exclusion is indicated.

\section{Ohio State University}

Columbus, OH 43210

\section{In vivo Analysis of Archaeal Transcription Signals and the Regulation of Heat Shock Promoters \\ C. Daniels, Department of Microbiology \\ $\$ 96,969$}

We are examining the transcription apparatus of the Archaea using the model organism, Haloferax volcanii. A plasmid based in vivo transcriptional reporter system has been developed to investigate the requirements for transcription initiation and transcription 
termination in this organism. Mutational analysis of the TATA-like element of the $H$. volcanii tRNALys gene indicates a close correlation between the archaeal and eucaryal sequence requirements for unregulated gene expression. Having defined the requirements for basal transcription these studies are now being extended to examine the requirements for regulated gene expression. The $H$. volcanii TF55/TCP1-like genes were chosen as model for regulated gene expression. Preliminary results indicate that the signals for heat shock regulation lie within the region of the TATA element. This type of regulation differs from the typical eucaryal heat shock regulation scheme. Development of a transcription termination assay has allowed us to investigate the requirements for transcription termination. Current results indicate that T-tract sequences are efficient terminators, whereas bacterial rho-independent terminators are ineffective. Methods are now available to determine the precise requirements for termination. The study of the $H$. volcanii transcription system has been extended to include an analysis of transcription factor genes. We have characterized three genes encoding the eucaryal-like TATA binding protein (TBP) in $H$. volcanii and Northern analysis indicates that these genes are differentially expressed. Similarly, we have characterized six eucaryal-like TFIIB genes in $H$. volcanii. Like the TBP genes, these genes also exhibit differential expression, with specific TFIIB genes responding to heat shock stress and entry into stationary phase. Despite the clear relation of archaeal and eucaryal transcription machineries, the multiplicity of transcription factors and their differential expression suggest a novel mode of gene regulation in the halophilic Archaea, transcription factor pairing.

\section{Ohio State University Columbus, $\mathrm{OH} 43210$}

\section{Mechanisms of Microbial Adaptation \\ C. Daniels and W. Strohl, Department of Microbiology}

Graduate programs'specializing in Microbial Biology have declined in recent years, greatly reducing the opportunities for advanced training in this important research area. As one mechanism to rebuild interest in Microbial Physiology and to train new scientists, we have developed a four week summer course focusing on the theme "Mechanisms of Microbial Adaptation". The course contains both lecture and laboratory components targeted to senior level graduate students and industrial scientists who desire an in-depth introduction to microbial physiology research. Current topics in the mechanisms of microbial adaptation will be presented in lectures by the faculty participants and these will be supplemented with presentations by experts in the field. Lectures will address: adaptive response of microorganisms in fermentations, global response mechanisms, regulation of microbial autotrophism, carbon metabolism in methanogens, and biodegradation and bioconversion by microorganisms. The accompanying laboratory exercises will illustrate current practical problems in the topic areas and introduce the students to modern experimental techniques and conceptual approaches. These experiments will provide the students with experience in fermentation technology, characterization and quantitation of proteins and metabolic intermediates, enzyme activity measurements and basic kinetic analyses, genetic approaches 
for the identification of regulated genes, and the anaerobic techniques needed to purify oxygen-sensitive proteins. Laboratory sessions will prepare the students to challenge and expand their current research and to undertake new approaches in their experimental systems.

A second inherent goal of the course is to establish a significant interactive community of research scientists in the field of Microbial Physiology. Introducing students to leading scientists in an informal but intense science setting creates a sense of excitement and community.

\section{Ohio State University Columbus, $\mathrm{OH} 43210$}

\section{Biosynthesis of Hydrocarbons}

P.E. Kolattukudy, Neurobiotechnology Center, Medical Biochemistry Department $\$ 96,000$ (FY 95 funds/2 years)

Hydrocarbons are found in most organisms in the animal and plant kingdoms. We showed that biosynthesis of hydrocarbons involves elongation of fatty acids to $C_{30}$ and $C_{32}$ level, reduction of the fatty acyl moiety to aldehyde followed by the loss of the carbonyl group with retention of the aldehydic hydrogen. Fatty acyl-CoA decarbonylase from the microsomes from A. porrum leaves has been solubilized and partially purified. The mechanism of the final step, decarbonylation of aldehyde, is under investigation. Fatty acids are reduced to alcohols for wax ester synthesis and to fatty aldehydes for hydrocarbon synthesis. Both types of reductases postulated to be involved in these processes were found in pea leaves that produce both hydrocarbons and wax esters. Purification of both reductases to homogeneity showed that the alcohol-generating enzyme is a $58 \mathrm{kDa}$ protein whereas the aldehydegenerating enzyme is only half as large. Similar reductases were detected in leek (Allium porrum) epidermis and the microsomes from housefly and fleshfly. The acyl-CoA reductase gene was cloned from Botryococcus braunii and this gene was sequenced.

CDNA for an epidermis-specific transcript from Senecio odora leaves was cloned and sequenced revealing an open reading frame that encodes a protein with seven transmembrane domains. Based on the homology of this protein with products of maize and Arabidopsis genes whose mutations affect cuticular wax synthesis, we postulate that this novel class of proteins is probably involved in intermembrane compartmental trafficking and deposition of cuticular waxes. This protein called EPI23 has been expressed for testing of its function. 
Ohio State University Columbus, $\mathrm{OH} 43210$

152. Transmethylation Reactions During Methanogenesis from Acetate or Methylamines in Methanosarcina barkeri

J.A. Krzycki, Department of Microbiology

$\$ 94,000$

We have been elucidating the mechanisms of methanogenesis from methylotrophic substrates, particularly methylated amines and thiols. These pathways represent interesting examples of highly regulated enzymes in archaebacteria, as well as significant routes of methane formation in natural environments. Two different protein families are involved in these pathways: corrinoid binding proteins, which are methylated from the growth substrate; and the isozymes of methylcorrinoid:CoM methyltransferases (MT2) which interact with them. The methyl-CoM produced is then converted to methane.

Methanogenesis from trimethylamine (TMA), dimethylamine (DMA), and monomethylamine (MMA) involve an isozyme of MT2 called MT2-A. MT2-M, an isozyme specific for methanol catabolism, is also able to function in methane formation from TMA. MT2-A interacts with different corrinoid proteins which are specific for methanogenesis from TMA, DMA, or MMA. The corrinoid protein of the MMA pathway is a $29 \mathrm{kDa}$ corrinoid protein which was first detected following its methylation in cell free extract following inhibition of methanogenesis. The MMA corrinoid protein and MT2-A were both isolated as two proteins which stimulate methyl-CoM formation from MMA in cell extracts. The methylation of CoM by TMA has been reconstituted with three purified proteins: a $26 \mathrm{kDa}$ corrinoid protein, a larger protein whose role we are investigating, and either MT2-A or MT2-M. We are currently isolating the proteins involved in DMA dependent CoM methylation, and completing reconstitution of the MMA pathway for comparative studies of these enzymes.

$M$. barkeri uses polypeptides similar to the methylamine enzymes to methylate CoM with the methanogenic substrates dimethylsulfide or methylmercaptopropionate. A single $480 \mathrm{kDa}$ corrinoid protein, first discovered by it's methylation when methanogenesis is inhibited, catalyses CoM methylation with either methylated thiol. The protein's larger subunit is a novel member of the MT2 family. The smaller subunit is a corrinoid protein with the B12 binding motif of bacterial and mammalian methionine synthase. This corrinoid binding subunit and the methylamine corrinoid proteins are also members of a homologous family. These results have provided a model for the interaction of the corrinoid proteins and MT2 homologues with this important coenzyme of methanogenesis. 


\section{Ohio State University Columbus, $\mathrm{OH}$ 43210-1096}

\section{The Molecular Characterization of the Lignin-Forming Peroxidase \\ L.M. Lagrimini, Department of Horticulture and Crop Science \\ $\$ 100,000$}

We are defining the role of the tobacco anionic peroxidase in growth, development, and host defense with particular attention to its participation in lignification. The overexpression of the anionic peroxidase in transgenic plants consistently resulted in increased lignification; however, antisense transformed plants did not have lower lignin content. This result suggests that the anionic peroxidase may not be the only enzyme in tobacco which participates in developmental lignification. In contrast, we have observed that the anionic peroxidase does play a significant role in host defenses - perhaps through the deposition of lignin-like polymers. Transgenic plants overexpressing this enzyme were found to have improved disease and insect resistance. Antisense transformed plants were found to be more susceptible to insect damage. Localization studies showed that the anionic peroxidase is expressed at the highest levels in the epidermis and trichomes - the first line of defense. We have also shown that the endogenous levels of this enzyme can have dramatic effects on the growth and development of the plant. Everything from the plant's growth rate, time-to-flowering, and root/shoot ratio to it's response to gravity, cold, and touch is effected in transformed plants with altered peroxidase activity. Under ideal growing conditions antisense transformed plants actually grow more vigorously than controls; however, these plants are highly sensitive to any number of external stresses. Experiments with the purified enzyme indicate its activity towards the phytohormone indole-3-acetic acid. Our current hypothesis regarding this enzyme is that its primary role is in host defense; however, because of its activity towards IAA, there is some compromise in vigor under optimal growing conditions.

\section{Ohio State University Columbus, $\mathrm{OH} 43210$}

\section{Structure and Regulation of Methane Genes J.N. Reeve, Department of Microbiology $\$ 269,660$ (FY 95 funds/18 months)}

The goal of this project is to determine how the seven-step, hydrogen-dependent pathway of carbon dioxide reduction to methane is regulated in the moderately thermophilic methanogen, Methanobacterium thermoautotrophicum (Mt). All of the enzymes and their encoding genes are now known, and several steps in this pathway are catalyzed by pairs of functionallyequivalent enzymes or isoenzymes. The availability of hydrogen, the source of energy for $\mathrm{Mt}$, determines which of the alternative pairs of encoding methane genes are expressed, and this, in turn, apparently results in a cellular metabolism tailored alternatively for maximum growth rate or maximum growth yield. Methanobacterium thermoformicicum (Mf) a very close relative 
of $\mathrm{Mt}$, can also grow and generate methane from formate under an atmosphere of nitrogen, and hydrogen availability has been shown to also regulate the expression of the genes that encode formate dehydrogenase in Mf, the enzyme that facilitates the growth of Mf on formate. The molecular mechanisms by which environmental hydrogen and formate levels are sensed, communicated intracellularly and interpreted into the activation and inactivation of different methane gene promoters, are currently under investigation. Mt DNA-dependent RNApolymerase and recombinant transcription factors are being used to mimic and dissect this regulation in vitro. Transformation protocols, with several different antibiotic resistance and metabolic selections are being evaluated as genetic approaches to deciphering the physiological and environmental regulation of methane biosynthesis in vivo.

\section{Ohio State University Columbus, $\mathrm{OH} 43210$}

\section{Photosynthetic Electron Transport in Genetically Altered Chloroplasts R.T. Sayre, Departments of Biochemistry and Plant Biology $\$ 107,000$}

Light induced charge separation by the photochemically active chlorophyll(s) of photosystem II (PS II) generates one of the most oxidizing potentials in nature. The oxidized PS II reaction center chlorophyll(s) in turn drive the oxidation of water producing molecular oxygen. Charge separation in PS II is one of the largest of all biochemical processes, however, the structure and function of the PS II complex remains to be resolved. Structural models of the photosystem II complex have been developed based on predicted and resolved structural similarities between the PS II complex and the bacterial photosynthetic reaction center crystal structure. We are testing these PS II structural models by targeting select amino acid residues in the PS II D1 and D2 proteins for site directed mutagenesis followed by phenotypic characterization of the mutants. Our objectives are to identify and characterize residues which: 1) are involved in binding and orientation of chromophores, and 2) participate in or regulate charge transfer processes. We are generating these mutations in the chloroplast genome of the single celled alga Chlamydomonas reinhardtii. Previous studies have demonstrated that Chlamydomonas has many advantages as a genetic and biochemical system for the characterization of PSII. Furthermore, Chlamydomonas serves as a model system for the genetic manipulation of higher plant chloroplasts. 
Ohio State University

Columbus, $\mathrm{OH}$ 43210-1292

\section{Regulation of Alternative $\mathrm{CO}_{2}$ Fixation Pathways in Prokaryotic and Eukaryotic Photosynthetic Organisms F.R. Tabita, Department of Microbiology \\ $\$ 100,000$}

Photosynthetic microorganisms employ diverse systems to reduce and assimilate carbon dioxide and in many instances $\mathrm{CO}_{2}$ serves as an extremely important electron sink for the dissipation of excess reducing power. The major goal of this project is to determine how photosynthetic microorganisms regulate the assimilation of $\mathrm{CO}_{2}$ in the absence of the CalvinBenson-Bassham (CBB) pathway and develop alternatives to remove excess reductant generated by photosynthesis and cell metabolism. This is particularly relevant in mutant strains of nonsulfur purple photosynthetic bacteria (Rhodospirillum rubrum and Rhodobacter sphaeroides), where the $\mathrm{CBB}$ reductive pentose phosphate pathway, the major $\mathrm{CO}_{2}$ reduction and carbon acquisition pathway, is blocked. We have uncovered discrete molecular signals that control the expression of genes required for alternatives to the CBB cycle for the removal of excess reducing power, and we have shown that all of the reducing pathways are tightly regulated and coordinately controlled. A two-component signal transduction system was identified, involving a sensor kinase and a response regulator, that appears to direct and coordinate the expression of structural genes for each of the reducing pathways. Current studies are directed at elucidating more of the molecular events involved in the development of alternatives to the CBB cycle and in identifying additional molecular links that must come into play. These studies are aided by the great metabolic diversity of the nonsulfur purple bacteria and the fact that these organisms possess easily manipulated genetic systems. We have also directed our attention to an examination of the molecular regulation and biochemistry of the key enzymes of the reductive tricarboxylic acid (RTCA) cycle, a $\mathrm{CO}_{2}$

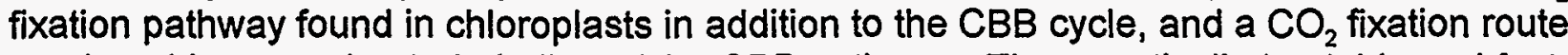
employed by some bacteria in lieu of the CBB pathway. The genetically tractable and fastgrowing green sulfur photosynthetic bacterium, Chlorobium tepidum, a moderate thermophile, has served as a model for our studies of the RTCA pathway. We have purified two of the key and unique enzymes, pyruvate synthase and ATP-citrate lyase, and we are engaged in isolating the structural genes and in determining how the RTCA path might be regulated in this organism. A number of autotrophic negative mutants have been isolated that grow only in the presence of organic carbon; these strains should greatly facilitate and complement the molecular studies. 


\section{Oklahoma State University Stillwater, OK 74078-3035}

\section{The Structure of Pectins from Cotton Suspension Culture Cell Walls A.J. Mort, Department of Biochemistry and Molecular Biology

In work over the last ten years on pectins from cotton suspension culture cell walls we have concluded that pectins are based on only two backbone structures. One is a (1-4) linked galacturonan and the other is a repeated disaccharide of galacturonic acid and rhamnose. The complexity of pectins is likely to arise from variations in the length of the uninterrupted stretches of these two structures and variations in sugar sidechains and acylations attached to them. The homogalacturonan based pectin regions fall into three common types: 1) True homogalacturonans with varying degrees of methyl esterification, 2) Rhamnogalacturonan II, a homogalacturonan section with complex sidechains clustered such that it can be isolated as an 5,000 Dalton fragment after endopolygalacturonase digestion of cell walls, and 3) xylogalacturonan, a homogalacturonan backbone with frequent single xylose substituents linked to the galacturonic acid residues. The rhamnogalacturonan is heavily acetylated and seems to contain regions with long sidechains attached to the rhamnose residues and other regions with single galactose residues attached to the rhamnose residues. The goal of the project is to learn more about the structure, and if possible, function of the various pectic regions and to determine which regions are covalently attached to each other. We also want to determine how they are linked to each other by isolating and characterizing junction zones between them. To help achieve these goals we will be developing highly sensitive methods for carbohydrate analysis and sequencing using capillary electrophoresis, enzyme and chemical digestions, and spectroscopic methods.

\section{University of Oklahoma Norman, OK 73019-0245}

\section{Energetics and Kinetics of Syntrophic Aromatic Degradation M.J. Mclnerney, Department of Botany and Microbiology

The syntrophic, benzoate-degrading bacterium, strain SB, in coculture with a sulfate-reducer or a methanogen degraded benzoate to a threshold concentration. The benzoate threshold concentration was not caused by nutritional limitations, the loss of metabolic activity, inhibition by elevated levels of hydrogen or formate, or acetate toxicity. The calculated changes in free energy for the sulfate reducer coculture $(-30.1 \mathrm{~kJ} / \mathrm{mol})$ and the methanogenic coculture $(-32.4$ $\mathrm{kJ} / \mathrm{mol}$ ) when the benzoate threshold was reached were nearly identical. Since the calculated changes in free energy for the sulfate-reducing and the methanogenic cocultures were nearly identical and independent of the electron-accepting process and the type of hydrogen-using organism present, we propose that a critical Gibb's free energy value may exist that represents the energetic minimum at which substrate degradation can occur. 
Strain SB is the first syntrophic bacterium known to use both benzoate and fatty acids for growth in association with hydrogen/formate-using bacteria. SB can also be grown in pure culture with crotonate. Phylogenetic analyses placed SB in the delta class of the Proteobacteria. SB represents a new species, Syntrophus acidotrophicus, in the genus Syntrophus. The enzyme activities detected in cell extracts of $S$. acidotrophicus are consistent with a pathway for benzoate metabolism where benzoate is activated to benzoyl-CoA by a ligase reaction and then transformed, in a series of reactions via cyclohex-1-ene-carboxyl-CoA, pimelyl-CoA, and glutaryl-CoA, to three molecules of acetate and one molecule of carbon dioxide.

\section{Oregon Graduate Institute of Science \& Technology Portland, OR 97291-1000}

\section{Biochemical Genetics of Lignin Degradation by Phanerochaete chrysosporium \\ M.H. Gold, Department of Chemistry, Biochemistry, and Molecular Biology}

The objective of this research is to further our understanding of the lignin degradative system of the basidiomycete Phanerochaete chrysosporium. We are using a variety of biochemical and molecular biological approaches to characterize the enzymes and genes involved in this process.

We are studying the structure and mechanism of lignin and manganese peroxidases (LiP and $\mathrm{MnP}$ ) via spectroscopic, kinetic, and bioorganic methods. We have developed an efficient homologous expression system that produces recombinant $\mathrm{MnP}$ in $P$. chrysosporium under the control of a primary metabolic gene promoter, facilitating structure/function studies via site-directed mutagenesis. Using this system, we have isolated and characterized by kinetic, spectroscopic, and crystallographic methods several mutations in the Mn binding site of $\mathrm{MnP}$. Studies on a wide variety of other $\mathrm{MnP}$ mutants are underway. A similar system is being developed for LiP.

We are characterizing an intracellular quinone reductase which is involved in the further degradation of monomeric quinones produced as lignin degradation products. We have isolated the gene encoding the intracellular quinone reductase of $P$. chrysosporium, and a variety of studies are planned to determine the role of this quinone reductase in lignin degradation. Genes encoding enzymes involved in $\mathrm{H}_{2} \mathrm{O}_{2}$ metabolism also are being isolated and characterized. 


\section{Oregon Graduate Institute of Science \& Technology Portland, OR 97291-1000}

160. Cellobiose Dehydrogenase and $\beta$-Glucosidase from Phanerochaete chrysosporium: Effect on Cellulose Hydrolysis, Cloning, and Characterization

V. Renganathan, Department of Chemistry, Biochemistry, and Molecular Biology $\$ 88,000$ (FY 95 funds/17 months)

Cellobiose dehydrogenase (CDH) is an extracellular hemoflavoenzyme produced by cellulose-degrading cultures of fungi such as Phanerochaete chrysosporium, Sporotrichum thermophile, and Coniophora putaeana. In this study, two genomic clones of $\mathrm{CDH}$, isolated from a $\lambda E M B L 3$ library of $P$. chrysosporium, will be sequenced and analyzed. Cellulose-degrading cultures of $P$. chrysosporium produce a novel cellulose-binding, extracellular $\beta$-glucosidase, which is presumably organized into a cellulose-binding domain and a catalytic domain. A cDNA clone of this glucosidase will be isolated from a cDNA expression library of $P$. chrysosporium and sequenced. The proposed experiments will help in understanding the structure of $\mathrm{CDH}$ and $\beta$-glucosidase from $P$. chrysosporium.

\section{Oregon State University Corvallis, OR $97331-2902$}

161. Catalytic Mechanism of Hydrogenase from Aerobic $\mathbf{N}_{\mathbf{2}}$-Fixing Microorganisms D.J. Arp, Laboratory for Nitrogen Fixation Research

$\$ 84,000$

We are investigating the catalytic mechanism of the hydrogenase from the aerobic, $\mathrm{N}_{2}$-fixing microorganism, Azotobacter vinelandii. This enzyme efficiently recycles the $\mathrm{H}_{2}$ evolved by nitrogenase. Several properties of this hydrogenase (e.g., low rate of the back reaction, $\mathrm{H}_{2}$ evolution, and a low $\mathrm{Km}$ for $\mathrm{H}_{2}$ ) make it ideal to function in a environment in which all of the available substrate is generated in situ. This enzyme is a Ni- and Fe-containing dimer with subunits of molecular weight 65,000 and 35,000 and is typical of a large group of $\mathrm{H}_{2}$ oxidizing hydrogenases. We are using mutagenesis of the genes coding for the subunits of hydrogenase to investigate the roles of specific amino acid residues in catalysis. Replacing the ligands to the FeS clusters results in proteins with either no activity or reduced activity. We are investigating the ligands to $\mathrm{Ni}$ and determining if these altered proteins without activity lack Ni. Following random mutagenesis, we are attempting to select hydrogenases with an altered ratio of $\mathrm{H}_{2}$ evolution to $\mathrm{H}_{2}$ oxidation. Finally, we are investigating the role of $\mathrm{HoxZ}$. This putative cytochrome is believed to be involved in transfer of electrons from hydrogenase to the electron transport chain. We are using site-specific mutagenesis to produce proteins with alterations in the putative heme binding site. The results are expected to provide a more complete understanding of the mechanism of hydrogenase. 


\section{University of Oregon Eugene, OR 97403}

\section{Genetic Analysis of Chloroplast Translation \\ A. Barkan, Institute of Molecular Biology $\$ 150,000$ (FY 95 funds/18 months)}

The components of the photosynthetic apparatus accumulate in the appropriate ratios and within the correct cell types as a consequence, in part, of the regulated expression of chloroplast genes. This project pertains to the control of chloroplast protein synthesis by nuclear-encoded factors, few of which have been identified. The project is focused on crp1, a nuclear gene in maize. Previous results established a role for crp1 in controlling the translation of the petA mRNA and in the metabolism of petB and petD mRNAs. Thus, the crp1 gene activates the expression of a small set of chloroplast genes. We recently cloned the crp1 gene by taking advantage of the Mu transposon tag. The sequence of a full length cDNA clone revealed no significant homologies with proteins in GenBank. The cDNA is being used to generate recombinant protein for immunolocalization studies and for assays of biochemical function. The role of crp1 in activating translation of its target mRNAs will be investigated by defining the step at which translation is blocked in mutant chloroplasts, identifying RNA binding activities that interact with target mRNAs in a genotype-dependent fashion, determining whether the crp1 gene product is a component of these binding activities, and assessing activities of purified crp1 protein. Understanding the mechanism by which crp1 activates translation will further our understanding of chloroplast translation in general. Most primary transcripts in the chloroplasts of higher plants are subject to endonucleolytic processing. In crp1 mutants, the absence of monocistronic petD mRNA correlated with a loss of petD translation, suggesting that petD is inefficiently translated in a polycistronic context. Further experiments will explore the role of endonucleolytic RNA processing in controlling the translation of chloroplast mRNAs. Finally, Mu populations will be screened for new mutants with defects in the translation of chloroplast mRNAs.

\section{Pennsylvania State University University Park, PA 16802}

163. The Characterization of Psychrophilic Microorganisms and Their Potentially Useful Cold-Active Glycosidases

J.E. Brenchley, Department of Biochemistry and Molecular Biology $\quad \$ 99,493$

Our objective is to characterize psychrophilic microorganisms and to discover, purify, and study novel cold-active glycosidases. Cold-active glycosidases with high activities at low temperatures can be used for low temperature conversions of saccharides found in plant biomass, paper-pulping waste, whey, etc. into chemical fuels or fermentation media. Comparisons of cold-active enzymes with their corresponding proteins from higher temperature organisms will yield insight into structural features involved in thermal stability. 
Our goals are to complete the characterization of three B-galactosidase isozymes cloned from a single Arthrobacter strain, to isolate new psychrophiles producing cold-active glycosidases with even lower temperature optima, to clone and sequence genes for selected enzymes, and to purify cold-active glycosidases for biochemical studies and comparisons with other enzymes. We have completed the analysis of the three Arthrobacter genes. One encodes a large $111 \mathrm{kDa}$ subunit similar to the lacz family, except the enzyme has a temperature optimum about $20^{\circ} \mathrm{C}$ below that of the Escherichia coli enzyme. The enzyme encoded by the second gene helped define a new class, which we designated lacG. The third gene encodes a small $52 \mathrm{kDa}$ subunit with homology to the lysosomal acid B-galactosidases found in human and mouse. The enzyme is a dimer and hydrolyses both $B-1,3$ and $B-1,4$ linkages. Further examination of this protein may provide insight into the evolution of these small glycosidases.

\section{Pennsylvania State University University Park, PA $16802-4500$}

\section{Light-Energy Transduction in Green Sulfur Bacteria \\ D.A. Bryant, Department of Biochemistry and Molecular Biology . $\quad \$ 78,000$}

The long-term, objective of this research program is to develop a detailed understanding of the structure, function, and biogenesis of the light-energy transduction apparatus found in green sulfur bacteria. Secondary goals are to analyze their transcriptional machinery, to develop methods for genetic analyses, and to understand the relationship of green sulfur bacteria to other eubacteria. Genes encoding eight (of ten) Chlorobium tepidum chlorosome proteins have been cloned, sequenced, and characterized. Five chlorosome proteins have been overproduced in $E$. coli, purified, and used to raise high-titer polyclonal antisera. Using these antisera as probes, the CsmA, CsmB, CsmC, CsmD, and CsmE proteins were shown to be surface-exposed components of the chlorosome envelope. Genes encoding CsmH, $\mathrm{Csml}$, and CsmJ have also been characterized. The latter two proteins are similar in their $\mathrm{N}$-terminal domains, and each protein contains a cysteine-containing sequence motif suggesting each will contain a [2Fe-2S] cluster. We have confirmed that both $\mathrm{C}$. tepidum and C. vibrioforme strain $8327 \mathrm{D}$ are naturally transformable and that homologous recombination can be used to target interposons into chromosomally encoded genes. Mutants harboring an omega-cartridge insertion in the csmC gene have been confirmed by Southern blotting and immunoblotting with antiserum to $\mathrm{CsmC}$. The mutants assemble chlorosomes but grow more slowly than the wild-type strain and appear to be defective in energy transfer. Finally, we have isolated and sequenced genes encoding sigma factors from a diverse collection of phototrophs. The data have been used to establish phylogenetic relationships among eubacteria and the results compared to those for other molecular markers such as 16S rRNA. 


\section{Pennsylvania State University University Park, PA 16802}

\section{Biophysical and Molecular Mechanisms Controlling Plant Cell Growth D.J. Cosgrove, Department of Biology $\$ 107,500$}

Plant cell expansion requires controlled stress relaxation and surface expansion of the tough cell wall. These processes are thought to depend on the combined activity of wall "loosening" and "rigidification" proteins, and also involve a rapid feedback loop to regulate the wall expansion process. We are taking several approaches to elucidate the processes underlying cell wall enlargement. Using a novel method to modulate plant growth rate with a pressure chamber, we are characterizing the rapid feedback circuit and its alteration in growth mutants. Along more molecular lines, we have detected developmentally-regulated changes in wall "rigidity", as assayed by the wall's extension response to applied expansins. We have also found evidence for a wall-bound enzymatic activity that modifies the wall so it becomes insensitive to expansin action. This "rigidification" activity is mimicked by crude pectin esterase preparations. We are testing the hypothesis that pectin de-esterification by pectin esterase inhibits growth in vivo. This involves (a) analysis of pectin esterase and pectin esterification during developmental and stress-related changes in growth, (b) tests of the growth effect of pectin esterase application to excised tissues, and (c) genetic alteration of pectin esterase activity in transgenic plants. Finally, in a more theoretical vein, we are developing a biophysical model of cell wall expansion based upon the thermodynamics of hydrogen-bonded polymer networks. From these theoretical and experimental studies we hope to identify the biophysical and molecular mechanisms that enable plant cell walls to expand during growth and to resist expansion upon maturation.

\section{Pennsylvania State University University Park, PA 16802}

\section{Role of $\mathrm{Ca}^{++} / \mathrm{calmodulin}$ in the Regulation of Microtubules in Higher Plants R. Cyr, Department of Biology $\$ 89,000$}

The cytoskeleton, and in particular its microtubule component, participates in several processes that directly affect growth and development in higher plants. Normal cytoskeletal function requires the precise and orderly arrangement of microtubules into several cell cycle and developmentally specific arrays. One of these, the cortical array, is notable for its role in somehow directing the deposition of cellulose, the most prominent polymer in the biosphere. It is therefore important to acquire information regarding the molecules which regulates microtubules within the different arrays. Experimental data has been obtained to suggest that plant cells use calcium, in the form of a $\mathrm{Ca}^{++} /$calmodulin complex, to affect the dynamics of microtubules within the cortical array. Owing to the importance of $\mathrm{Ca}^{++}$as a regulatory ion in higher plants we are probing for a putative $\mathrm{Ca}^{++} /$microtubule transduction 
pathway which may serve to integrate microtubule activities within the growing and developing plant cell. We have found that elongation factor 1-a behaves as a microtubule associated protein serving to both stabilize, and bundle, microtubules in vitro. Both of these effects are modulated in vitro by $\mathrm{Ca}^{++} /$calmodulin which unbundles and destabilize microtubules. Our working hypothesis is that similar events occur in vivo and we are currently undertaking a structure/function approach to identify the regions on this MAP that are involved in these activities. This information will, in turn, be used to develop strategies for specifically purturbing this iteration.

\section{Pennsylvania State University University Park, PA 16802-4500}

\section{Enzymological Studies of One-Carbon Reactions in the Pathway of Acetate Utilization by Methanogenic Bacteria \\ J.G. Ferry, Department of Biochemistry, Microbiology and Molecular and Cell Biology

Several enzymes in the pathway for the fermentation of acetate to methane and carbon dioxide have been purified from Methanosarcina thermophila and are under investigation utilizing biochemical, biophysical and molecular genetic approaches. Acetate kinase (Ack) and phosphotransacetylase (Pta) catalyzes the activation of acetate to acetyl-CoA. The catalytic mechanisms of both enzymes are being investigated with site directed mutagenesis experiments utilizing the recombinant enzymes. Essential glutamate, arginine, and histidine residues have been identified for Ack which support a proposed mechanism involving a phosphorylated enzyme intermediate. An essential cysteine residue in the Pta was identified which is consistent with a mechanism in which cysteine extracts a proton from CoASH producing the thiolate anion for attack on acetyl phosphate. The proposed mechanisms are expected to extend to homologous enzymes from several diverse anaerobes. A novel ironsulfur flavoprotein (Isf) was characterized which is involved in the electron transport pathway required for the fermentation of acetate. Characterization of the redox centers is underway utilizing electron paramagnetic resonance spectroscopy in collaboration with Dr. Steve Ragsdale. Site-directed mutagenesis experiments with recombinant Isf have identified residues which coordinate the iron-sulfur center. Identification of additional electron transfer proteins and cofactors continues. 


\section{Pennsylvania State University University Park, PA 16802}

\section{Molecular-Genetic Analysis of Maize Starch Branching Enzyme Isoforms} M. Guiltinan, Department of Horticulture

Starch is an important energy storage molecule used by all plants. Its synthesis involves the creation of branch points by a class of enzymes known as starch branching enzymes (SBE, EC 2.4.1.18). Biochemical studies have shown that three isoforms of SBE exist in maize endosperm, SBEl, lla and Illb.

We estimate that the SBEs are encoded by two to three genes each for SBEI and IIb. Although we have made significant progress in the isolation of CDNA and genomic clones encoding SBEl and IIb, to fully understand the role of the SBE isoforms in starch biosynthesis, a comprehensive molecular-genetic dissection of the SBE gene family is underway. The specific objectives of this project are to: 1) Isolate CDNA clones encoding maize SBElla. 2) Isolate and characterize CDNAs and/or genomic clones representing each of the SBE family members not yet isolated. 3) Identify maize lines with mutations in each of the SBE genes. To reach these Objectives, we are using a combination of conventional molecular techniques to isolate the genes, together with a reverse genetics approach that will result in identification of maize lines with mutations in each of the SBE genes. Together, these lines of research will advance our knowledge of the importance of the SBEs in starch biosynthesis and in the life cycle of the plant. The knowledge gained and the tangible products of this research (genes, cDNAs and novel germplasm) may also be useful in the production of starches with altered structure for use in industry.

\section{Pennsylvania State University University Park, PA 16802}

\section{Characterization of Lignin and Mn Peroxidases from Phanerochaete chrysosporium \\ M. Tien, Department of Biochemistry and Molecular Biology

Lignin accounts for approximately $20 \%$ of the Earth's biomass. It is predominantly degraded by filamentous fungi. Our research is focused on the enzymology of lignin degradation by the white-rot fungus Phanerochaete chrysosporium. This fungus secretes two isozyme families, the lignin and Mn peroxidases. These enzymes catalyze the oxidation of lignin via a free radical mechanism and low molecular weight redox mediators. The role of these two enzymes and the mediators in lignin biodegradation is the focus of our research. We are addressing this question by characterizing the enzymology and regulation of these isozymes in $P$. chrysosporium. The genes encoding these enzymes have now been expressed in bacteria and refolded to yield active enzyme. Site-directed mutagenesis along with kinetic 
and spectroscopic studies are aimed at determining the structural aspects which confer these peroxidases with their unique reactivities. We are also attempting to use these genes to generate mutants lacking lignin or Mn peroxidase activity to determine the role of these genes in lignin degradation.

\section{University of Pennsylvania \\ Philadelphia, PA 19104-6018}

\section{Structural Basis of Signal and Energy Transduction in Plants A.R. Cashmore, Plant Science Institute, Department of Biology

Through this program we are training graduate students in both structural and molecular genetic studies in plant science. Students are being trained in graduate programs affiliated with both the Departments of Biology and Chemistry. These students are pursuing research in a wide variety of topics including: the role of the virA gene product in the transfer of T-DNA from Agrobacterium into plant cells; the crystal structure of carbonic anhydrase; chemical synthesis of taxol; and the vacuolar sequestration of cadmium in plants. A very successful retreat was organized in which investigators from the Chemistry, Biochemistry/Biophysics, and Biology Departments presented recent findings from their research. By this mechanism students are being trained with an appreciation of both molecular genetic as well as chemical, biochemical, and biophysical approaches to research in plant science.

\section{University of Pennsylvania \\ Philadelphia, PA 19104-6018}

\section{Light Responses and Photoperiodism in Arabidopsis thaliana A.R. Cashmore, Plant Science Institute, Department of Biology

We have characterized a collection of early flowering mutants for defects in either phytochrome signalling and/or circadian rhythms. The pef mutants (phytochrome/early flowering) show early flowering under both long and short days, and are affected in a number of phytochrome-mediated responses. Three complementation groups of pef mutants have been identified. pef1 is deficient in both red and far-red inhibition of hypocotyl elongation and cannot be rescued by biliverdin. Western blot and difference spectrum analysis indicate appreciable concentrations of photochemically active phytochrome A. pef1 is therefore a good candidate for an early signal transduction mutant that affects both PhyA and PhyB-mediated responses. pef2 and pef3 appear impaired in red light, but not far-red light inhibition of hypocotyl elongation and may therefore be more specific to PhyB-mediated events. In addition to the pef mutants, we have isolated a class of mutants with an alteration in the critical photoperiod mediating the delayed flowering response. Wild-type Arabidopsis shows pronounced delay in flowering if grown under an 8-hour photoperiod. We have 
isolated a class of mutants that demonstrates this delay in flowering only under a 6-hour, or shorter, photoperiod. Three complementation groups were identified, all of which are incompletely dominant and show no other morphological or developmental abnormalities. Examination of the circadian rhythms in these mutants by following the cycling of CAB gene expression revealed that one of these mutants had a shortened period length of approximately two hours, in good agreement with the alteration in the critical photoperiod of this mutant. We postulate this may be a mutant within some component of the circadian clock.

\section{University of Pennsylvania Philadelphia, PA 19104-6018}

\section{Membrane-Attached Electron Carriers in Photosynthesis and Respiration F. Daldal, Department of Biology $\$ 195,600$ (FY 95 funds/18 months)}

The overall aim of our project is the molecular characterization of the structure, function and biogenesis of membrane-associated cytochromes (cyt) that act as electron carriers in photosynthesis (Ps) and respiration (Res). In these studies we are using the facultative photosynthetic bacterium Rhodobactercapsulatus as a model system forenergy transduction. The presence of a dual electron transfer (ET) pathway between the cyt $b c_{1}$ complex and the reaction center in Ps, and the cyt $c$ oxidase in Res, was first indicated by our Ps- and Res-proficient mutants lacking the soluble cyt $c_{2}$. We have since then demonstrated that a novel membrane-associated cyt, cyt $c_{y}$ is the molecular basis of this entirely membrane-confined ET pathway that functions both in Ps and Res growth conditions, as indicated by appropriate mutants. We have also studied various $R$. sphaeroides strains harboring cyt $c_{y}$ to determine its ET properties in respect to its heterologous physiological partners. Using an epitope-tagged and functional derivative of cyt $c_{y}$ we purified it to define its physicochemical properties and its mode of attachment to the membrane. These studies now define the membrane topology of cyt $c_{y}$, and reveal that it is composed of an "anchor-linker" and a "cyt $c$ " domains. We were able to show that the former domain can also anchor the soluble cyt $c_{2}$ to the membrane in a functional form. This approach is now providing an interesting opportunity for the first time to study the ET properties of any given cyt (e.g., cyt $c_{2}$ ) in both soluble and attached form. We are also continuing our studies on how the membrane-bound c-type cytochromes are biosynthesized, and in this respect, have recently characterized a new mutant that led us to $\mathrm{cycH}$, a gene whose product is essential for the biogenesis and maturation of various $c$-type of cytochromes. 


\section{University of Pennsylvania \\ Philadelphia, PA 19104-6018}

\section{Molecular and Genetic Analysis of Hormone-Regulated Differential Cell Elongation in Arabidopsis \\ J.R. Ecker, Department of Biology \\ $\$ 213,000$ (2 years)}

The goal of this project is to understand how hormones act to regulate differential elongation of plant cells. The plant growth regulators ethylene and auxin are known to modulate cell elongation in the hypocotyl and apical hook in etiolated seedlings; the apical hook being formed by asymmetric elongation of cells on opposite sides of the hypocotyl. The identification of mutants in Arabidopsis that exhibit altered patterns of differential growth in the hypocotyl is being carried out. One such class of mutants lack an apical hook; the hookless mutants. The HOOKLESS1 gene has been cloned and its predicted translation product shows similarity to $\mathrm{N}$-acetyltransferases. Notably, expression of HLS1 mRNA was found to be modulated by ethylene; thus providing a link between the auxin and ethylene response pathways. In order to further define its function in the regulation of differential cell elongation, biochemical characterization of the HLS1 protein will be carried out. Additionally, characterization of a novel differential growth mutant called hookless 4 and cloning of the HOOKLESS 4 gene is also planned. Through the identification of mutant Arabidopsis plants which show defects in differential growth in the hypocotyl and by characterization of their corresponding gene products, we hope to gain new insight into the little understood process of differential cell elongation. It is expected that the general mechanisms found to be essential for controlling cell growth in this model system will be utilized in other plant tissues and in agriculturally important crop plants.

\section{University of Pennsylvania Philadelphia, PA 19104-6018}

\section{Structure-Function Analysis of Vacuolar $\mathrm{H}^{+}$-Pyrophosphatase P.A. Rea, Department of Biology}

Plant cells contain alternate metabolic pathways that utilize nucleoside triphosphates or inorganic pyrophosphate (PPi) as energy sources. The full significance of this phenomenon remains to be determined but the use of these two energy currencies is exemplified by the presence of two $\mathrm{H}^{+}$pumps on the membrane bounding the central vacuole of higher plant cells. These are the vacuolar $\mathrm{H}^{+}$-ATPase (V-ATPase; EC 3.6.1.3), an enzyme common to the endomembranes of all characterized eukaryotes, and a vacuolar $\mathrm{H}^{+}$-translocating inorganic pyrophosphatase (V-PPase; EC 3.6.1.1), which is ubiquitous in plants but otherwise known in only a few phototrophic bacteria. Both enzymes pump $\mathrm{H}^{+}$from the cytosol to vacuole lumen to establish a $\mathrm{H}^{+}$gradient that is employed to energize the transport of a broad range of solutes across the vacuolar membrane. 
Our research is directed at understanding the structure and function of the V-PPase at the protein level through the application of biochemical and molecular techniques. The basis of the research underway is our recent success in cloning cDNAs encoding the V-PPase from Arabidopsis thaliana and its isoforms from Beta vulgaris, reconstituting partially purified preparations of the enzyme to generate transport-competent proteoliposomes in vitro and our ability to heterologously express CDNAs encoding mutated forms of the V-PPase from Arabidopsis in the yeast Saccharomyces to yield membrane fractions containing appreciable quantities of functional pump.

Having defined the C-terminal topology of the enzyme and demonstrated a specific requirement for cytosolically oriented $\mathrm{Cys}^{634}$ for inhibition by maleimides but the dispensability of all of the conserved Cys residues, including Cys ${ }^{634}$, for V-PPase function, our studies are now aimed at determining the involvement of acidic amino acid residues located within or near transmembrane spans in substrate turnover, $\mathrm{H}^{+}$-translocation and/or activation by monovalent cations. To date we have identified three main types of mutant enzyme: those in which PPi hydrolysis is decoupled from $\mathrm{H}^{+}$translocation; those that have been rendered insensitive to DCCD; and, those that have altered cation selectivity. One member of the first class of mutants, E427Q, though still active in PPi hydrolysis, mediates $\mathrm{H}^{+}$-translocation at less than $6 \%$ of the wild type rate to yield an 8- to 9-fold diminished coupling ratio. While it may be premature to conclude that these results demonstrate a direct role for $\mathrm{Glu}^{427}$ in $\mathrm{H}^{+}$ transfer, the recovery of wild type $\mathrm{H}^{+}$pumping versus the modest increase in PPi hydrolytic activity by a Glu $\rightarrow$ Asp substitution does nonetheless imply an important role for an acidic residue at this position for $\mathrm{H}^{+}$-translocation per se. Most of our current research efforts are therefore concerned with further defining the functional significance of $\mathrm{Glu}^{427}$ for transmembrane $\mathrm{H}^{+}$movement in the context of our revised model for the organization of the enzyme.

Its novel origins, membership of a new category of ion translocase and utilization of such a simple phosphoanhydride as energy source, make the V-PPase a unique system for examining the mechanistic basis of energy-dependent ion translocation across biological membranes.

\section{Purdue University \\ West Lafayette, IN 47907}

\section{Purification and Molecular Cloning of the Synthases of Cereal $(1 \rightarrow 3),(1 \rightarrow 4)-\beta-d-$ glucan \\ N.C. Carpita, Department of Botany and Plant Pathology $\$ 96,000$}

We continue our search in maize for the genes that encode the Golgi-localized synthases of the $(1 \rightarrow 3),(1 \rightarrow 4)-\beta$-d-glucan ( $\beta$-d-glucan), a cell-wall polysaccharide unique to the cereal 
grasses. This cell wall polysaccharide is unique to the cereal grasses and has recently gained attention for its possible role in lowering serum cholesterol. In grasses, the $\beta$ - $\alpha$-glucan plays an essential role in the growth of primary tissues. Absent from cell walls of dividing cells, the $\beta$-d-glucan accumulates specifically during cell expansion. After expansion, they decrease markedly in proportion to other polymers. During grain development, the $\beta$-d-glucan appears again as major constituents of the endosperm wall. The general structure is a polymer of cellotriosyl and cellotetraosyl units connected by single $(1 \rightarrow 3)-\beta$-d-linkages. Contiguous $(1 \rightarrow 3)$ linkages and longer stretches of $(1 \rightarrow 4)$ linkages, which are susceptible to cleavage by specific endo-glucanohydrolases, are also built into the polymer at special points. We have enriched membranes of the Golgi apparatus from maize (Zea mays L.) by a single-step flotation gradient and used them to synthesize in vitro a $\beta$-d-glucan identical in structure to the native cell-wall polymer (Gibeaut, D. M., and N.C. Carpita, 1993, Proc. Natl. Acad. Sci. USA 90, 3850-3854). We also observed a callose synthase activity that co-localized with the Golgi apparatus and did not require calcium activation. Because of parallel activities and enzyme characteristics between cellulose and $\beta$-d-glucan synthesis in vitro, we propose that the genes that encode the cereal $\beta$ - $d$-glucan synthases are derived from ancestral cellulose synthase genes. We will use maize cDNAs with high homology to the UDP-Glc binding domain of the cotton CelA gene, which likely encodes the cellulose synthase catalytic subunit, to select fulllength cDNAs corresponding to the maize CelA genes. Experiments are designed to differentiate between cellulose synthase and $\beta$-d-glucan synthase by: a) differential expression in seedling and endosperm, and $b$ ) the immunolocalization of unique peptides of the $\beta$-d-glucan and cellulose synthases at the Golgi or plasma membrane, respectively. We have discovered several Golgi intrinsic-membrane proteins that may also function in the synthase or in the secretory pathway, and we will continue to purify these polypeptides for microsequencing, antibody production, and selection of their full-length cDNAs in expression libraries.

\section{Purdue University \\ West Lafayette, IN 47907-1153}

\section{Modification of Lignin Composition in Plants by Manipulation of ferulate-5- hydroxylase Expression \\ C.C.S. Chapple, Department of Biochemistry

Our goal is to understand key factors that determine the quality and quantity of lignin in plants, and ultimately to address this economically important issue by the directed manipulation of lignification. Lignin in angiosperms is largely derived from ferulic acid and sinapic acid and the ratio of the monomers derived from these precursors has an impact on lignin degradability. We hypothesized that ferulate-5-hydroxylase (F5H) expression may regulate lignin monomer composition because this enzyme catalyzes the first of the two steps that convert ferulic acid to sinapic acid. If so, modification of $\mathrm{F} 5 \mathrm{H}$ expression would be a valuable tool with which to modify lignin composition in plants. In order to address this question, we used the fah 1-2 mutant of Arabidopsis to identify cDNA and genomic clones for 
$\mathrm{F} 5 \mathrm{H}$. By overexpression of $\mathrm{F} 5 \mathrm{H}$ in transgenic Arabidopsis, we have demonstrated that $\mathrm{F} 5 \mathrm{H}$ is an important regulatory site governing lignin composition. We have now begun to characterize the expression of $\mathrm{F} 5 \mathrm{H}$ using northern analysis and $\mathrm{F} 5 \mathrm{H}$-reporter gene fusions in relation to sinapic acid ester biosynthesis and lignification.

\section{Purdue University}

West Lafayette, IN 47907

\section{A Genetic Analysis of the Lumenal Proteins of the Photosystem II $\mathrm{O}_{2}$-evolving Complex in Cyanobacteria \\ L.A. Sherman, Department of Biological Sciences

The primary objective of this project will be to determine the proteins and protein domains in the cyanobacterium Synechocystis sp. PCC6803 that interact with the Mn-stabilizing protein (MSP) and help form the lumenal component of the PSII $\mathrm{O}_{2}$-evolving complex. We will first clone and sequence the genes encoding two small lumenal proteins that have been implicated as potential replacements for MSP. These are psbV (a.k.a. petK), coding for cytochrome $\mathrm{C} 550$, and $p s b U$, coding for the $12 \mathrm{kDa}$ protein. We will also isolate mutations in other PSII genes that affect the lumenal side of PSII. We will concentrate on MSP and the lumenal domains of CP47. The screening will use a Digital Imaging Spectrometer (DIS), which permits many colonies to be analyzed for fluorescence or absorption properties simultaneously. This screening approach will allow us to perform a variety of random and site-specific mutagenesis procedures. We have obtained several interesting mutants via random mutagenesis of the $p s b O$ gene. Over $75 \%$ of the colonies selected by DIS screening have been confirmed by DNA sequencing as psbO mutants. These mutants have interesting physiological properties; some are similar to our previous site-directed mutants, whereas many are different.

We are also analyzing the oxidizing side of PSII in the unicellular, diazotrophic cyanobacterium Cyanothece sp. ATCC 51142. This strain shows many alterations in PSII when grown under $\mathrm{N}_{2}$-fixing conditions and the oxidizing side of PSII is clearly a target for down-regulation of $\mathrm{O}_{2}$-evolution. Analyses of this strain will be valuable for an understanding of the regulation of $\mathrm{O}_{2}$-evolution in photosynthesis. 


\section{Rice University \\ Houston TX 77005}

\section{Genetic and Biochemical Analysis of Solvent Formation in Clostridium acetobutylicum \\ G.N. Bennett and F.B. Rudolph, Department of Biochemistry and Cell Biology}

$\$ 135,000$ (FY 95 funds/18 months)

The anaerobic organism Clostridium acetobutylicum has been used for commercial production of important organic solvents due to its ability to convert a wide variety of crude substrates to acids and alcoholș. The objectives are to improve our knowledge of the molecular genetics and enzymology of Clostridia in order to make genetic alterations which more effectively channel cell metabolism toward production of desired products. Two factors that limit butanol production in continuous cultures are: 1) The degeneration of the culture, with an increase in the proportion of cells which are incapable of solvent production. Isolated degenerate strains are being evaluated to analyze the molecular mechanism of degeneration. Recent studies show two general types of degenerates, one which has lost essential solvent pathway genes and a less complete type which has not lost all solvent production capability and retains the DNA bearing solvent pathway genes. 2) The production of hydrogen which uses up reducing equivalents in the cell. If the reducing power could be more fully directed to the reduction reactions involved in butanol production, the process would be more efficient. We have studied oxidation reduction systems related to this process. We have also analyzed some genes related to ATP production and membrane energetics.

\section{Rice University \\ Houston, TX 77251}

\section{A Molecular-Genetic Approach to Studying Source-Sink Interactions in Arabidopsis thaliana$$
\text { S.I. Gibson, Department of Biochemistry and Cell Biology }
$$

Sources (such as leaves) and sinks (such as roots and seeds) of fixed carbon interact in complex ways that play an important role in plant development and physiology as well as in determining crop yields. The mechanisms by which sources and sinks of fixed carbon interact are poorly understood, but are likely to involve the regulation of key genes by soluble sugar levels. One aspect of our research is focused on determining how pervasive sugar-regulated gene expression is in plants. A second aspect of our research is focused on elucidating the molecular mechanisms by which sugar-regulated gene expression occurs in plants. This goal is being accomplished by identifying and characterizing mutants in the model plant Arabidopsis thaliana that are defective in sugar-regulated gene expression. This work should lead to a better understanding of source-sink interactions, which is a pre-requisite to developing more rational approaches to improving crop yields. 
The final aspect of our work is focused on characterizing fumaric acid accumulation in a variety of plants, including important crop plants such as wheat, rice, cotton, bean, tomato, sunflower and corn. Our interest in this area stems from our discovery that fumaric acid accumulates to very high levels in at least some plants. Fumaric acid is of interest because it can be used by plants to synthesize sugar and starch. Therefore, understanding fumaric acid accumulation could be critical to understanding sugar and starch metabolism in plants, a topic of great economic importance to agriculture.

\section{Rice University Houston, TX 77005}

\section{Structural Studies of Archaeal Thermophilic Adenylate Kinase J. Konisky, Department of Biochemistry and Cell Biology}

Recent studies of thermophilic and hyperthermophilic members of the Archaea have begun to suggest biochemical and physiological strategies by which these organisms are able to survive, indeed, thrive at temperatures that approach, and, in some cases, exceed the boiling point of water. The objective of our research program is to delineate the structural features that confer thermostability to adenylate kinase (ADK), an enzyme that catalyzes the interconversion of adenylate nucleotides. We have isolated the ADK's from four phylogenetically-related marine methanogenic members of the Archaea; the mesophile $M$. voltae (growth temperature, 35 to $40^{\circ} \mathrm{C}$ ), the thermophile, $M$. thermolithotrophicus $\left(65^{\circ} \mathrm{C}\right.$ ) and the hyperthermophiles, $M$. jannaschii and $M$. igneus ( 85 and $88^{\circ} \mathrm{C}$, respectively). Comparative analysis and modeling studies based on the known structure of previously characterized ADK's have led us to make presumptive identification of structural features that we propose contribute to the temperature stability as well as the optimal catalytic temperature for each enzyme. These hypotheses are being tested through a combination of physical and structural studies that include CD, DSM and fluorescence spectroscopy. X-ray crystallographic studies are also underway to solve the structure of each enzyme in the set. So as to test our hypotheses, genetically engineered proteins are being analyzed for their catalytic and structural properties. It is expected that our studies will contribute to the basic understanding of protein stability, particularly as it relates to enzymes that function near $100^{\circ} \mathrm{C}$. 


\section{University of Rochester \\ Rochester, NY 14627-0166}

\section{Synergism and Interaction Between Clostridium thermocellum Major Cellulosome Components, CelS and CelL \\ J.H.D. Wu, Department of Chemical Engineering

Enzymatic cellulose degradation is a heterogeneous reaction requiring binding of soluble cellulase molecules to the solid substrate. Based on our studies of the cellulosome, a cellulase complex, we have previously proposed that such binding can be brought about by a special anchorage subunit. In this anchor-enzyme model, Cell (the largest subunit of the cellulosome) enhances the activity of CelS (the most abundant catalytic subunit of the cellulosome) by anchoring it to the cellulose surface. We have subsequently reported that CelS contains a conserved duplicated sequence at its $C$-terminus and that CelL contains nine repeated sequences with a cellulose-binding domain (CBD) in between the second and the third repeats.

In this work, we reexamined the anchor-enzyme mechanism using recombinant CelS and various CelL domains, CBD, R3 (the repeat next to CBD), and CBD-R3, expressed in Escherichia coli. As analyzed by non-denaturing gel electrophoresis, $\mathrm{rCelS}$, through its conserved duplicated sequence, formed a stable complex with R3 or CBD-R3, but not with CBD. Although R3 or CBD alone did not affect the binding of rCelS to cellulose, such binding was dependent on CBD-R3, indicating the anchorage role of CBD-R3. Such anchorage apparently increased the $\mathrm{rCel}$ (S activity toward crystalline cellulose. These results substantiate the proposed anchor-enzyme model and the expected roles of individual CelL domains and the conserved duplicated sequence of CelS. They shed more light into the cellulosome mechanism. As this intriguing and novel anchor-enzyme mechanism continues to unveil, it may be possible to use the concept to engineer enzymes for biotechnology applications.

\section{Rockefeller University New York, NY 10021}

182. Characterization of the Systemic Acquired Resistance Immediate-Early
Response to Salicylic Acid
N.-H. Chua, Laboratory of Plant Molecular Biology

We have been investigating the role of salicylic acid (SA) in plants during systemic acquired resistance to pathogens by characterizing the genes and signal transduction components involved in the cycloheximide-insensitive immediate-early (IE) response. Our efforts to date have revealed 16 tobacco genes that are induced within the first 2 hours following SA treatment. These genes fall into approximately four discrete classes of responses, varying in their sensitivity to SA concentration and cycloheximide, their mRNA induction and decay 
kinetics, and their overall mRNA abundance. As predicted, some of these sequences show similarity to transcription factors and signal transduction components, as well as to some functions not previously demonstrated to be regulated by $S A$, such as a UDP-glucose:flavonoid glucosyltransferase. Many of these genes may be induced in response to pathogen, methyl jasmonate, auxin, or hydrogen peroxide treatment, whereas others show greater specificity. Two genes were found to be induced by very low concentrations of SA. In additional studies, we have characterized SARP, a protein which interacts with the Cauliflower Mosaic Virus 35S promoter as-1 element in an SA-induced manner. It was found to be sequestered in an inactive complex in the absence of SA, which could be released following SA treatment in a phosphorylation dependent manner or by dissociating agents. Finally, we have designed novel vectors for glucocorticoid-inducible target gene expression.

\section{Rutgers University New Brunswick, NJ 08903}

\section{Molecular Analysis of a Thylakoid $\mathrm{K}^{+}$Channel}

C. Chin (for G.A. Berkowitz), Department of Plant Science

$\$ 171,100$ (FY 95 funds/18 months)

Ion channels are a class of membrane proteins which are very important for the metabolic functioning of cells. Because they are extremely low abundance proteins, they are very difficult to purify; no ion channel has ever been purified (and sequenced) from a plant membrane. We have developed a novel probe which can be used to identify $\mathrm{K}^{+}$channel proteins. This probe is an antibody generated against the conserved pore region of $\mathrm{K}^{+}$ channels. The research rationale of this project is to use this anti-pore antibody to facilitate the first-ever purification of an intracellular plant $\mathrm{K}^{+}$channel protein. The target membrane for this work will be the thylakoid membrane of the chloroplast; the most ubiquitous membrane on earth, which is also easy to purify in large quantities. $\mathrm{A} \mathrm{K}^{+}$channel is present in the thylakoid membrane; the function of this protein is critical for maximal photosynthetic activity. We will use the antibody to immunoprecipitate the thylakoid $\mathrm{K}^{+}$channel. Partial peptide sequencing of the immunoprecipitated $\mathrm{K}^{+}$channel will allow for specific probes to be developed which will then be used to identify a cDNA encoding the polypeptide. Sequencing of the cDNA will lead to the molecular characterization of this $\mathrm{K}^{+}$channel protein. Biochemical analyses will also be undertaken to characterize the native function of the thylakoid membrane $\mathrm{K}^{+}$channel. 


\section{Rutgers University}

New Brunswick, NJ 08903-0231

\section{Respiration and Active Oxygen Species Production in Stressed Plants I. Raskin, AgBiotech Center

Plants respond to a variety of stresses by changing their respiratory metabolism. For example, we have observed that chilling stress results in the activation of the cyanide-insensitive or alternative respiratory pathway (AP) in the leaves of a variety of plant species. We suggest that the increased "heating" of the inner mitochondrial membrane at low temperatures is accomplished by a larger electron flux through the AP, which dissipates most of the energy stored in the respiratory substrates as heat. This localized thermoregulation counteracts the deleterious effects of chilling on the fluidity of the mitochondrial membrane, which determines the activity of the respiratory enzymes vital for plants.

In addition to chilling, pathogenesis is another stress associated with increases in both cyanide-sensitive and cyanide-insensitive respiration. Increased respiration in the infected tissues is associated with a substantial increase in heat production. We will test the hypothesis that active oxygen species generated as a result of the increased respiration in plant leaves undergoing a hypersensitive response initiate the cascade of molecular events which directly leads to the increased expression of defense-related proteins. We will also assess whether salicylic acid, a known inducer of plant respiration, is the cause or the result of the respiratory burst and active oxygen species production observed during the incompatible plant-pathogen interaction. The involvement of respiration in the generation of active oxygen species will be also investigated.

\section{Rutgers University \\ Piscataway, NJ 08855-0759}

\section{Corn Storage Protein: A Molecular Genetic Model}

Changes in the amino acid composition of the seed of the crop that is used world-wide in the production of meats and cultivated fish could eliminate the manufacture of essential amino acids by fermentation. Essential amino acids are not absent in cereal seeds, but their relative accumulation compared to other amino acids dictate the level of costly supplements in the diet of animal feed. Even protein-rich crops like soybeans used in these diets to make up for deficiencies in corn do not accumulate enough essential amino acids. The reason for this is the different amino acid composition of seed proteins and how their synthesis is regulated during plant growth after fertilization. However, we have shown in animal feeding trials that the regulation of a single gene like dzs10 can not only be used to change the amino acid composition of corn meal, but also have a direct impact on animal growth. To determine the 
physiological role of this gene, a gene knock-out mutant caused by a transposable element belonging to a new member of the "CACTA" family of transposable elements has been characterized. Homozygous plants do not show any discernible kernel phenotype different to overproducers. Therefore, traditional plant breeding techniques fail to yield superior varieties. However, knock-out mutants can be used to identify sequence elements and factors that achieve the desired balance in gene expression.

\section{Salk Institute for Biological Studies La Jolla, CA 92037}

\section{Signal Transduction Pathways that Regulate CAB Gene Expression J. Chory, Plant Biology Laboratory

The process of greening, or chloroplast differentiation, involves the coordinate regulation of many nuclear- and chloroplast-encoded genes. The cues for the initiation of this developmental program are both extrinsic (e.g., light) and intrinsic (cell-type and plastid signals). Several regulatory photoreceptors are involved in the perception of light signals; however, the exact mechanisms by which light and other signals are perceived by plant cells and converted into physiological responses are not understood.

The research program focuses on the genetic, biochemical, and molecular characterization of new Arabidopsis thaliana mutants that have been previously isolated in our laboratory. We have utilized a molecular genetic approach to select for Arabidopsis mutants in which a photoregulated promoter $(C A B)$ is expressed aberrantly with respect to light, intrinsic developmental signals, and signals from the chloroplast. These genetic screens have allowed us to identify 18 new genes that play a role in the signal transduction pathways controlling photoregulated gene expression and chloroplast development in Arabidopsis. Specifically, we have obtained mutants in which $C A B$ is expressed either: (1) in the light at abnormally low levels, defining positive regulators (9 genes); $(2)$ in the dark at abnormally high levels, defining negative regulators ( 3 genes); or (3) in the presence of inhibitors that eliminate chloroplast function, defining an intracellular signaling pathway from chloroplasts to the nucleus ( 6 genes). Experiments are underway to clone several of these positive and negative regulatory genes. The proposed experiments will further our knowledge of light-mediated signal transduction pathways, and will be a step toward elucidating how information is transmitted from photoreceptors and organelles in the cytoplasm to regulatory factors in the nucleus. 


\section{The Scripps Research Institute La Jolla, CA 92037}

\section{Genetic Engineering with a Gene Encoding a Soybean Storage Protein R.N. Beachy, Department of Cell Biology

Regulation of the family of genes that encode the $\beta$-conglycinins, the seed storage protein of soybean (Glycine max) is primarily at the level of gene transcription, although there is evidence of post-transcriptional regulation in some members of the gene family. Our research is directed to the identification of nucleotide sequences and protein factors that are involved in regulating the expression of members of this gene family. Recently we completed the analysis of transgenic tobacco plants that contain chimeric genes comprised of sequences $5^{\prime}$ and $3^{\prime}$ of the genes encoding the $\alpha^{\prime}$ - and $\beta$-subunits of $\beta$-conglycinin and the reporter gene, uidA. The results of these studies clearly demonstrated that temporal regulation of expression of the chimeric gene (during the development of the immature embryo) is controlled by sequences $5^{\prime}$ of the gene, while the amount of protein product is influenced by nucleotide sequences $3^{\prime}$ of the coding sequence.

To identify the proteins, referred to as the soybean embryo factors (SEF proteins), that bind to nucleotides $5^{\prime}$ of the coding sequence and the core promoter (i.e., $5^{\prime}$ of the TATA sequence), we employed a cloning vector derived from a yeast expression system. In this system nucleotide sequences proposed as important for binding of SEF proteins were ligated upstream of a yeast core promoter ligated with a selectable marker. Cloned cDNA derived from embryo mRNAs was ligated with a plasmid to construct fusion proteins with the activator domain of the yeast promoter. Colonies were identified that contain sequences encoding a protein that binds the TATA element of the $\alpha$ '-promoter; and another that binds a sequence upstream of the TATA sequence previously implicated in regulating expression of the promoter. The sequence of the cloned CDNA was determined and the derived protein sequence provides information about the nature of the putative regulatory protein. We will continue our analysis of this protein by developing antibodies and relevant nucleotide probes to localize expression of the gene in developing embryos.

\section{The Scripps Research Institute}

\section{La Jolla, CA 92037}

\section{Membrane Targeting of P-type ATPases in Plant Cells} J. Harper, Department of Cell Biology

Our long term goal is to understand how integral membrane proteins are targeted to different subcellular locations. This knowledge is needed to engineer plants with new solute transport systems. Such systems include pumps for heavy metal uptake and salt efflux, which may be useful for bioremediation and salt tolerance, respectively. The general focus of our research 
is on a family of ion pumps called P-type ATPases. We are carefully examining the subcellular localization of two proton pumps (AHA2 and AHA10), two calcium pumps (ACA2 and $A C A 3)$ and a putative copper pump (AXA2) in a model plant system, Arabidopsis. Our approach is to use immunocytology and cell fractionation. The primary focus is on an unusual pump, ACA2, which is most similar to a plasma membrane-type calcium pump in animals, but is distinct since it is localized to an internal membrane (probably plastids). We are testing three hypotheses using mutant pumps expressed in transgenic plants. 1) Does the $\mathrm{N}$-terminal domain contain information required to target ACA2 directly to a plastid? 2) Does the tonoplast become a "default" destination when normal targeting information is deleted? 3 ) Can ACA2 be retargeted to the plasma membrane by making chimeras with different regions of a plasma membrane proton pump? This research provides the first investigation into how a P-type ATPase is targeted to a plastid. This pathway is separate from the ER-derived vesicular trafficking pathway which appears to localize all other eukaryotic P-type ATPases.

\section{The Scripps Research Institute La Jolla, CA 92037}

\section{Nuclear Genes Regulating Translation of Organelle mRNAs} S. Mayfield, Department of Cell Biology

We have identified and purified a set of four proteins that bind with high specificity and affinity to the $5^{\prime}$ untranslated region (UTR) of the chloroplast psbA mRNA. We have identified two independent biochemical mechanisms by which the binding activity of these proteins is regulated: ADP-dependent phosphorylation and thioredoxin mediated reduction. Using site-directed mutagenesis and chloroplast transformation we have identified a region of the 5' UTR of the psbA mRNA that is recognized by these RNA binding proteins, and that functions as a component of psbA translational activation. We have mapped the secondary structure of this RNA element by chemical and enzymatic probing. We have cloned a CDNA and genomic clone for one of the psbA RNA binding proteins $(47 \mathrm{kDa})$. This protein has a high degree of homology with poly(A) binding proteins, which have been shown to be involved in translational regulation in several other systems. We will isolate and characterize each of the other psbA RNA binding proteins (RBPs), produce antisera against these proteins, and isolate and characterize CDNA and genomic clones for each of these. We will define the specific RNA elements contained with the PsbA 5' UTR, and characterize the RNA secondary structure within these elements. Finally we will undertake a molecular genetic analysis of the psbA RBP genes as a means to define specific roles for each of the psbA RBPs. These sets of experiments should allow us to precisely define the factors that are involved in translational regulation of psbA mRNA, and provide important insight into the molecular mechanism by which protein/RNA interactions trigger translational activation of specific chloroplast mRNAs. 


\section{Medical University of South Carolina Charleston, SC 29525-2501}

190. Molecular Mechanisms Controlling Proton Pumping by Bacteriorhodopsin R.K. Crouch, Department of Ophthalmology and Biochemistry

Bacteriorhodopsin functions as a light-driven transmembrane proton pump. The protein is an integral membrane protein, having as its chromophore retinal which is bound to the protein via a protonated Schiff base, buried within the membrane. On absorption of light, a proton is translocated from the cytoplasmic side to the extracellular side of the membrane. The proton is released from the retinal Schiff base and eventually transferred to the extracellular membrane surface. Our studies have focused on understanding the mechanism of the proton release.

The proton released from the retinal Schiff base is transferred to D85. Recent studies by our group have demonstrated that an additional amino acid must be involved as a proton release group. Using mutants of R82, the biphasic nature of the titration curve of D85 was observed, which would be expected if there were another proton release group. This group has now been identified by Lanyi and his coworkers to be E204. Using mutants of both E204 and R82, our group has shown that normal proton release is obtained with $R 82 Q$ but not with E204Q, confirming that E204 is the proton release group. Our plans for the next year are aimed at understanding how the protonation states of R82 and E204 are related as the current model has them some distance apart. As we have recently had a breakthrough in mapping bR by mass spectrometry, this should facilitate our crosslinking studies aimed at probing the conformational changes which occur during the photocycle.

\section{Southern Illinois University Carbondale, IL 62901}

\section{Regulation of Alcohol Fermentation by Escherichia coli D.P. Clark, Department of Microbiology}

Our objective is to study the regulation of the synthesis of ethanol and lactate in the facultative anaerobe Escherichia coli. The protein encoded by the adhE gene expresses both alcohol and acetaldehyde dehydrogenase activities and is responsible for ethanol synthesis. Its $\mathrm{N}$-terminal half is homologous to other aldehyde dehydrogenases and its $\mathrm{C}$-terminus to $\mathrm{Fe}$-activated alcohol dehydrogenases. The adhE gene of Salmonella has been sequenced and while similar to that of $E$. coli, shows significant differences. The Salmonella enzyme has a broader substrate range and can use alcohols of six or more carbons in length, whereas the E. coli enzyme only works up to C4. The mechanism of anaerobic induction of adhE has been characterized by means of gene fusions. The build up of reduced NADH during anaerobic conditions appears to play the major role in regulating the adhE gene. Mutations 
resulting in constitutive expression of the adhE gene, even in alr are known. The upstream regions of several of these have now been sequenced, confirming that they are in the adhE promoter region. The regulation of adhE depends on the adh $R$ gene which we have recently cloned. Preliminary work indicates that the AdhR protein binds to DNA in the adhE upstream region. The IdhA gene, encoding the fermentative lactate dehydrogenase has also been cloned and sequenced. We have constructed gene fusions between IdhA and the lacZ gene. The mechanism of IdhA induction in response to acidic conditions is being investigated using these fusions. In strains deleted for both the IdhA and pta genes, neither lactic nor acetic acid can be produced anaerobically. Instead a novel fermentation product appears which is presently being characterized by NMR.

\section{Stanford University \\ Stanford, CA $94305-5020$}

\section{Nodulation Genes and Factors in the Rhizobium-legume Symbiosis S.R. Long, Department of Biological Sciences \\ $\$ 244,024$}

We study the interaction of Rhizobium meliloti and its legume host, alfalfa, which interact to form symbiotic nitrogen fixing root nodules. Our work will help improve use of symbioses as alternatives to use of ammonium nitrate chemical fertilizers, whose production is highly energy intensive. In particular, symbiotic nitrogen fixation provides nitrogen nutrition to the plant and thus allows it to synthesize protein in the absence of fertilizer. We study the Rhizobium (bacterial) genes that cause the plant to initiate symbiosis. These genes control synthesis of signals (Nod factors) that cause the plant host to form symbiotic root nodules. We are also searching for new bacterial genes important for symbiosis: we have recently begun the first steps of determining the sequence of the $1500 \mathrm{~kb}$ pSyma of $R$. meliloti, which may contain many new symbiosis genes. We have found a new gene, oppA, that appears to be important for survival of bacteria in stationary phase and is co-regulated with some symbiosis genes. Finally, we are developing methods of using microscopic observation to follow both the bacterial and plant cells during symbiosis. We are studying the early responses of the alfalfa host plant to Nod factors by electrophysiology and microscopy. We recently obtained the first microscopic evidence of calcium in root hairs responding to native Rhizobium symbiotic signals. Our further work will involve plant genetics as well as bacterial genetics and chemistry. We hope to understand what genes in the plant allow them to establish these useful and energy-conserving symbioses. 


\section{Stanford University \\ Stanford, CA 94305}

\section{Biochemical and Molecular Characterization of Enzymes for Cell Wall Synthesis \\ P.M. Ray, Department of Biological Sciences

Our laboratory is attempting to identify, and obtain genetic clones for, proteins responsible for enzymatic synthesis of cell wall polysaccharides (sugar polymers), which make up the substance of most of the renewable fuels available to mankind. We are currently working on plasma membrane-associated glucan synthase-II (GS-II, which forms $\beta-1,3$ glucan [callose] from UDPglucose) and on Golgi-associated GS-I (which forms from UDPglucose a $\beta-1,4$ glucan that is probably the backbone of Golgi-synthesized xyloglucan).

GS-II. By a complex purification procedure, together with direct photolabeling and antibody evidence, we can associate GS-II activity of pea seedlings with 2 polypeptides of 55 and 70 $\mathrm{kDa}$. We have cloned genes from a pea cDNA expression library by immunoscreening with antisera that we raised in rabbits against the $55-\mathrm{kDa}, 70-\mathrm{kDa}$ polypeptide bands. Each antiserum selected two different gene sequences, so each of these bands (55 and $70 \mathrm{kDa}$, respectively) evidently contains 2 polypeptides of about the same molecular mass. One of these sequences, in the case of both the $55-$ and the $70-\mathrm{kDa}$ bands, proved closely similar to the tonoplast ATPase (V-ATPase) subunit of the same molecular mass, which was surprising because the antigens were obtained from purified plasma membranes. The other sequence, in the case of the 70 , proved to be that for calnexin, a calcium-binding protein whose gene Delmer also recently obtained in trying to clone a GS-Il gene from cotton. In the case of the 55, the second clone has an entirely unique sequence (no match to anything in the gene sequence database). We think that this may represent the catalytic subunit of GS-II.

Immunological tests that we have made indicate that all of these polypeptides are probably part of a GS-II complex, and are not merely contaminants. Still further complexity has appeared through finding that besides the 55 and $70 \mathrm{kDa}$ polypeptides a previously unrecognized $220 \mathrm{kDa}$ polypeptide also correlates with GS-ll activity during fractionation. From immunological results and gene cloning it appears that this polypeptide is a covalently coupled complex of a 55 and a $70 \mathrm{kDa}$ subunit. The $55 \mathrm{kDa}$ member of this complex is the same V-ATPase subunit as is obtained separately as a free polypeptide during purification of GS-II. The $70 \mathrm{kDa}$ member, however, is a "heat-shock protein" called HSP 70. It appears that in the complete GS-II complex HSP 70 occurs only in covalently coupled association with the $55 \mathrm{kDa}$ V-ATPase subunit, and not also separately as a noncovalently associated subunit, as the $55 \mathrm{kDa}$ V-ATPase subunit apparently does.

The complexity that our results now indicate for the GS-Il enzyme complex is beginning to rival that of V-ATPase itself. What the functional meaning of this complexity is, and the reason why certain ATPase subunits, calnexin and HSP 70 are apparently a part of it, are important questions that we intend to investigate in the coming grant year. 
GS-I. By affinity chromatography we have isolated in pure form a polypeptide doublet of about $40 \mathrm{kDa}$ which we had previously found could be covalently labeled reversibly by glucose derived from UDPglucose. Various lines of evidence suggested that the $40 \mathrm{kDa}$ polypeptide (which we call the "doublet") might be part of the Golgi polysaccharide synthesizing system, but probably not the synthase GS-I itself.

By screening the pea cDNA library with an antiserum raised against the isolated $40 \mathrm{kDa}$ polypeptide we obtained a single class of gene clone, which has a unique sequence. By immunocytochemistry using this antiserum we have found that the $40-k D a$ polypeptide is specifically and dramatically localized to the trans part of pea (and also cotton) Golgi dictyosomes, and does not occur in ER, plasma membrane, other membranous organelles, or cytosol. Since the trans region of the Golgi stack has been identified as the site of xyloglucan synthesis, this finding strengthens the indications that the 40 is involved in polysaccharide synthesis. An important matter that we are working to try to accomplish, during the coming grant year, is a demonstration of whether UDPglucose-derived glucose that is carried by the doublet can be transferred to a polysaccharide chain.

We are also at work on isolating several minor polypeptides that we have found to correlate with GS-I activity during glycerol gradient fractionation. We intend to obtain partial amino acid sequences for these polypeptides, which can be used to design probes for isolating the corresponding genes which, in a coli expression system, will afford antigens for raising antisera that will be used to determine, by inhibition or adsorption of GS-I activity, if some of these polypeptides are part of a GS-I complex.

As a result of our recent work the glucan synthase problem has become much more complex than we anticipated.

\section{University of Tennessee Knoxville, TN 37996-0845}

\section{Plant Recognition of Bradyrhizobium japonicum Nod factors G. Stacey, Department of Microbiology $\$ \$ 140,000$ (FY 95 funds/18 months)}

We are studying the nitrogen-fixing symbiosis between Bradyrhizobium japonicum and soybean. B. japonicum infects soybean roots and induces the formation of a nodule, a new organ, in which the bacterium resides. Organogenesis of the nodule is induced by substituted lipo-chitin molecules synthesized by the products of the bacterial nodulation genes. Past work in our laboratory has focused on the chemical identification of these compounds and studies of their biosynthesis. More recently, we have been focusing on the plant response to the addition of the lipo-chitin nodulation signals. This work has revealed a surprising complexity in the mechanism involved in signal recognition. Our data indicate that Nod signal recognition is a two step process, with only the latter step showing structural specificity. Moreover, full 
nodule development requires the presence of at least two, structurally distinct lipo-chitin molecules. These studies suggest that the plant may possess two receptors that recognize distinct lipo-chitin signals. Our work has now focused on identifying these receptors. In collaboration with other laboratories, we have now identified a root specific lectin that recognizes the lipo-chitin molecules produced by $B$. japonicum and that possesses phosphatase activity. This molecule is a candidate for a Nod signal receptor. Our eventual goal is to elucidate the complete signal pathway involved in the soybean nodulation response. Detailed knowledge of legume symbioses is important for possible extension of biological nitrogen fixation for energy conservation.

\section{University of Tennessee \\ Knoxville, TN 37996-1100}

\section{Molecular Mechanism of Light-Regulated Protein Transport Across the Nuclear Envelope}

A.G. von Arnim, Department of Botany

$\$ 93,000$

The compartmentalization of eukaryotic cells into nuclear and non-nuclear domains affords a straightfonward means with which organisms can limit the access of transcriptional regulators to their nuclear target genes in order to control gene expression. However, little is known about the cellular mechanisms that regulate the nuclear protein uptake and depletion. Our preliminary studies have suggested that the Arabidopsis COP1 protein, a light inactivated repressor of transcription that plays essential roles during plant development, can confer a light regulated nuclear localization pattern on the fused reporter protein beta-glucuronidase (GUS). This project is.designed to reveal the molecular mechanism by which an environmental factor, light, directs the nuclear accumulation of a transcriptional regulator in the model plant species Arabidopsis thaliana. We are carrying out a three-pronged analysis of the molecular determinants in the COP1 protein that are required and sufficient for light regulated nuclear accumulation. Specific mutations are introduced into the COP1 protein; the mutant proteins are then examined for their ability to undergo light regulated nuclear localization and for their ability to support the essential functions of COP1 during plant development. Our mutational analysis focusses on conserved protein sequence motifs in COP1 and on the interaction of COP1 with plant kinases and other COP1-binding proteins. For the detection of COP1 protein mutants we are taking advantage of antibody-based methods as well as gene fusions to reporter proteins such as GUS and green fluorescent protein (GFP), which allows us to visualize specific proteins in living cells. 


\section{Texas A\&M University}

College Station, TX 77843-3122

\section{Role of Glycolytic Intermediates in Global Regulation and Signal Transduction \\ J.C. Liao, Department of Chemical Engineering \\ $\$ 101,000$}

Escherichia coli and other enteric bacteria regulate the assimilation of nitrogen through the Ntr regulon. A current theory states that $\alpha$-ketoglutarate and glutamine serve as signals for the availability of nitrogen. During nitrogen (ammonia) limitation, the intracellular glutamine concentration drops and the $\alpha$-ketoglutarate concentration increases because of less efficient synthesis of glutamate from glutamate dehydrogenase. The ratio of these two metabolites determines the level of NRI (NtrC) phosphorylation through actions of PII, UT/UR, and NRII (NtrB). However, the detailed mechanism of Ntr regulation is apparently much more complex than currently understood, and is likely to involve other metabolites as regulatory signals. To probe possible metabolite signals in vivo, we perturbed the metabolite pools by overexpressing enzymes which are directly or indirectly involved in the transformation of the metabolites of interest. It was found that overexpression of phosphoenolpyruvate (PEP)-forming enzymes such as PEP carboxykinase (Pck) or PEP synthase causes a Ntr-non-inducible phenotype in a pta strain, but not in the wild-type strains. This effect was attributed to the PEP-producing activity of the enzymes, because overexpression of inactive or altered-activity mutants did not cause the effect. Measurements of metabolite pools suggest that Fructose 1,6-diphosphate and pyruvate levels correlate with the non-inducible phenotype, suggesting possible roles of these metabolites as signals in the Ntr regulon.

\section{Texas Tech University Lubbock, TX 79409}

\section{Ferredoxin-Linked Chloroplast Enzymes}

D.B. Knaff, Department of Chemistry and Biochemistry

The ferredoxin-dependent glutamate synthase of spinach chloroplasts was shown to contain FMN and a [3Fe-4S] cluster as its sole prosthetic groups. Cyclic voltammetry indicated that the two groups are isopotential $\left(E_{m}=-225 \mathrm{mV}\right)$. The $S=0,[4 \mathrm{Fe}-4 S]^{2+}$ cluster of spinach chloroplast ferredoxin:thioredoxin reductase was examined by magnetic circular dichroism, resonance Raman and electron paramagnetic resonance spectroscopies. The cluster has cysteinyl sulfur ligands to all four cluster irons, cannot be reduced at ambient potentials down to $-650 \mathrm{mV}$, but can be oxidized to a $S=1 / 2[4 \mathrm{Fe}-4 \mathrm{~S}]^{3+}$ state $\left(E_{\mathrm{m}}=+420 \mathrm{mV}\right)$. These results argue against any role for this cluster in mediating electron transfer from ferredoxin $\left(E_{m}=-420\right.$ $\mathrm{mV})$ to the active site disulfide $\left(E_{m}=-230 \mathrm{mV}\right)$ of the enzyme. An alternative role for the cluster, i.e., stabilizing a thiyl radical formed by the initial one-electron reduction of the active site disulfide, was suggested by spectroscopic studies of a modified form of the enzyme in 
which one of the two active site cysteines had been alkylated. The alkylated enzyme exhibits an EPR signal with characteristics that point to the presence of a radical species coupled to a partially oxidized cluster. The species responsible for this signal undergoes a one-electron reduction $\left(E_{m}=-210 \mathrm{mV}\right.$ ) to a diamagnetic state. These results have been interpreted in terms of a [4FE-4S] $]^{3+}$ cluster covalently attached through a cluster sulfide to a cysteine-based thiyl radical involving one of the active site thiols.

\section{University of Texas}

Austin, TX 78713-7640

198. Molecular, Genetic, and Biochemical Analysis of Cellulose Synthesis in Arabidopsis thaliana

R.M. Brown, Jr., and K. Sathasivan, Department of Botany . $\quad \$ 89,000$

Membrane proteins from actively growing Arabidopsis were solubilized with increasing concentrations of digitonin and fractionated under native gel electrophoresis. Activities of native protein complexes in cellulose synthesis were visualized by incubation with UDP-glucose and activators, followed by staining with Tinopal. The products, two broad bands, correspond well with the protein bands revealed in the same gel with Commassie Blue. We developed a novel technique to remove the glucan products from the bands for TEM observations. $\beta-1,3$ and $\beta-1,4$ glucans were distinguished by $\mathrm{CBH}$ l-gold labeling. We are denaturing proteins from native bands with SDS to determine which polypeptides are associated with the products, to determine molecular weights, and to secure purified polypeptides for micro-sequencing and antibody preparation. We have developed a rapid assay procedure based on acetic-nitric acid treatment, using a microtiter plate to screen T-DNA tagged Arabidopsis mutants with altered cellulose content. Using this technique, we have identified putative mutants with reduced and increased cellulose content. These putative mutants currently are being characterized. We have designed degenerate primers based on the conserved sequences in bacterial cellulose synthases and related proteins. We are utilizing these primers to perform RT-PCR of Arabidopsis RNA. Products will be cloned and sequenced to identify the genes coding for cellulose synthase. Biochemical studies accompanied by cloning and characterizing the Arabidopsis genes involved in cellulose biosynthesis should facilitate cloning similar genes from economically important plants (cotton, trees) leading to crop improvement and better energy utilization for the future. 


\section{The Institute for Genomic Research Rockville, MD 20850-3319}

\section{Arabidopsis Genome Sequencing Using Random Shotgun Sequencing of BAC Clones}

J.C. Venter

$$
\$ 2,121,800 \text { ( } 2 \text { years) }
$$

The objective of this project is to produce high quality, annotated genomic sequence within the framework of the international Arabidopsis thaliana genome program. We will be employing two complementary BAC-based approaches to reach this goal. First, we will produce finished sequence from individual $B A C$ clones using a random shotgun approach that has been successfully used in recent bacterial genome projects. The sequence of each BAC clone will be annotated and submitted to GenBank. Second, we will sequence both ends of at least 8,000 clones from the two BAC libraries being used by other sequencing groups. This will provide the community with a sequence database that will facilitate the selection of a minimally overlapping BAC clone for any given sequence. By producing substantial amounts of high quality sequence and a resource that enables coordination of the final stages of sequencing the genome, we hope to make a critical contribution to the worldwide Arabidopsis community.

\section{Virginia Polytechnic Institute and State University Blacksburg, VA 24061}

\section{Enzymology of Acetone-Butanol-Isopropanol Formation} J.-S. Chen, Department of Biochemistry

Several species of Clostridium produce acetone, butanol and isopropanol (solvents), which are important industrial chemicals and fuel additives. Commercial production of solvents by fermentation began with the Weizmann process, using $C$. acetobutylicum and starchy raw materials. After the 1930s, C. acetobutylicum was replaced by newly isolated clostridia that produced high levels of solvents from sugars. In the past three decades, most historical strains of solvent-producing clostridia were labelled as $C$. acetobutylicum, but recent studies revealed significant differences between " $C$. acetobutylicum" strains. Based on DNA reassociation, we identified C. acetobutylicum, C. beijerinckii, and two other Clostridium species from currently available cultures of "C. acetobutylicum" and C. beijerinckii comprises about half of them. C. beijerinckij produces solvents from sugars without requiring $\mathrm{pH}$ control, and some strains produce isopropanol in addition to acetone and butanol. We have been studying the solvent-forming enzymes of $C$. beijerinckii with an aim of establishing mechanisms and strategies for regulating the expression of solvent-production genes and the flow of metabolites. The long-term goal is to prevent the degeneration of strains, to regulate the product ratio, and to be able to hold the cell in the solvent-producing state. Our present study has an emphasis on characterizing the aldehyde and alcohol dehydrogenases that 
convert butyryl-CoA to butanol, on determining the structure and organization of solventproduction genes, and to study the relationship between growth and solvent production. We are using a gene encoding a secondary alcohol dehydrogenase as a reporter gene to study the promoter regions for genes that are active in exponential, solvent-producing, and early stationary phases of growth.

\section{University of Virginia Charlottesville, VA 22901}

\section{Structural Domains In NADPH: Protochlorophyllide Oxidoreductases Involved in Catalysis and Substrate Binding M.P. Timko, Department of Biology

Chlorophylls play a fundamental role in the energy absorption and transduction activities of all photosynthetic organisms. In angiosperms, chlorophyll biosynthesis is regulated to a large extent by the light-dependent reduction of protochlorophyllide (Pchlide) to chlorophyllide (Chlide) catalyzed by a nuclear-encoded, plastid-localized enzyme known as NADPH: protochlorophyllide oxidoreductase (POR). In the presence of its substrates, Pchlide and NADPH, POR forms stable ternary complexes that accumulate in the prolamellar bodies of the etioplast of dark grown seedlings. Illumination of POR ternary complexes with stoichiometric amounts of light quanta result in the trans-reduction of the C17-C18 double bond in the $D$ ring of Pchlide and the formation of the Chlide product. The action spectra for the enzyme closely matches the absorption spectra for Pchlide indicating the Pchlide is the primary light-absorbing species involved in the reaction. In addition to its crucial regulatory role in chlorophyll formation, Pchlide reduction may also function as a light-regulated trigger controlling chloroplast development and potentially overall photomorphogenesis. We are interested in understanding the mechanism of light-dependent Pchlide reduction and the structural determinants in POR proteins required for substrate (NADPH and Pchlide) binding, catalytic activity, and proper intraorganellar assembly within plastid. Until recently little direct information was available about specific structural determinants of POR function. Using a novel bacterial expression system based on functional complementation of bacteriochlorophyll biosynthesis mutants of Rhodobacter capsulatus we have been able to characterize a series of site-directed and clustered charged-to-alanine scanning mutants of the pea POR protein for alterations in their ability to catalyze Pchlide photoreduction. Residues and/or domains thought to be essential for substrate binding and catalysis have been located and some of the factors required for proper plastid membrane integration have been determined. In addition, we have established a plausible model for the tertiary structure of the POR protein which allows us to be more predictive in our experimental approaches. We are currently attempting to clarify the role of the conserved residues TYR-275, LYS-279, and CYS-281 in the catalytic mechanism, as well as in identifying additional residues within the active site that may be directly involved in catalysis or important in either stabilizing the substrate (Pchlide) or enhancing the interaction of substrate and cofactor. We are also characterizing the reaction kinetics and structure of the active complexes in the wild-type and various POR mutants 
generated by alanine scanning mutagenesis in order to determine which property of the enzyme (i.e., $\mathrm{Km}$ for substrate or cofactor, turnover rate, etc.) was modified by the mutation(s). Based upon the findings of these experiments, we hope to develop a working model for the function and activity of this enzyme. These studies should provide significant information on one of the most crucial biosynthetic steps in the development of photosynthetic organisms.

\section{Washington State University Pullman, WA 99164-6340}

\section{Membrane Function in Lipid Mutants of Arabidopsis}

J. Browse, Institute of Biological Chemistry $\$ 265,490$ (FY 95 funds/18 months)

Our investigations of the biochemistry of wild-type and mutant plants have provided us with new information about the enzymology and regulation of lipid metabolism in higher plants. More importantly, our isolation and characterization of Arabidopsis mutants laid the ground work for important discoveries about the role of membrane lipids in the cell biology and physiology of plants. For example, a triple mutant line, fad3 fad7 fad8 lacks 18:3 the fatty acid precursor of jasmonate. Jasmonate is implicated in the signaling pathways that allow plants to defend themselves against chewing insects. We have therefore used the triple mutant to investigate the importance of jasmonate in plant defense. Mutant plants contained negligible levels of jasmonate and showed extremely high mortality (c. $80 \%$ ) from attack by larvae of a common saprophagous fungal gnat, Bradysia spp., even though neighboring wildtype plants were largely unaffected. Application of exogenous methyl jasmonate substantially protected the mutant plants and reduced mortality to c. $12 \%$. These experiments precisely define the role of jasmonate as being essential for the induction of biologically-effective defense in this plant/insect interaction.

\section{Washington State University Pullman, WA 99164-6340}

\section{Regulation of Terpene Metabolism}

R. Croteau, Institute of Biological Chemistry

Terpenoid oils, resins and waxes from plants are important renewable resources with a range of pharmaceutical, food and industrial uses. The objective of this project is to control terpenoid production by targeting regulatory steps for genetic engineering to increase the yields and expand the types of terpenoid natural products that can be made available for commercial exploitation. Model systems used are (+)-camphor metabolism in sage and $(-)$-menthone metabolism in mint. The pathways of biosynthesis and catabolism have been established, and the relevant enzymes have been purified and characterized. Developmental 
studies relating enzyme activity levels to terpene production within, and loss from, the secretory gland sites of metabolism suggest that terpene yield is largely controlled by the balance between biosynthetic and catabolic capacity. Antibodies directed against and cDNAs coding for enzymes presumed to represent rate-limiting metabolic steps have been prepared. Results obtained thus far using these experimental tools indicate that control of terpene biosynthesis resides primarily at the level of gene expression, that several compartments within the secretory gland cells (plastids, endoplasmic reticulum and cytosol) participate in terpene biosynthesis, and that the bulk of terpene production occurs at the early stages of gland development. Two genes have been selected for overexpression in transgenic plants to increase terpene yield, and the experiments to test this possibility are in progress.

\section{Washington State University Pullman, WA 99164}

\section{Carbon Metabolism in Symbiotic Nitrogen Fixation M.L. Kahn, Institute of Biological Chemistry}

$\$ 92,000$

Combined nitrogen is the nutrient that most often limits plant growth. For this reason, nitrogen fertilizer is an important input for increasing crop yield. However, producing nitrogen fertilizer is expensive and energy intensive and excess fertilizer can increase nitrate in groundwater and soil acidity. Some plants are able to obtain nitrogen through symbiotic associations with nitrogen-fixing bacteria in which the plant exchanges carbon compounds for ammonia produced by the bacteria. Enzymes of the plant and bacterial tricarboxylic acid (TCA) cycles are at the center of this exchange, generating energy, reductant and biosynthetic intermediates from the catabolism of photosynthate. We are investigating the genetics and biochemistry of symbiotic carbon metabolism in both soybean and alfalfa. Our immediate goal is to define the role of the decarboxylating leg of the TCA cycle, a series of reactions needed to synthesize amino acid precursors that may also be required to generate energy and reductant for nitrogen fixation. We are investigating TCA cycle mutants, including those with defects in citrate synthase, isocitrate dehydrogenase and oxoglutarate dehydrogenase, and are developing conditional TCA cycle mutants to probe the relationship between the TCA cycle and nodule development and metabolism. We are also investigating the molecular genetics of plant NADP-isocitrate dehydrogenases by studying several cDNAs we have cloned that code for various forms of the enzyme and studying the enzymes themselves using biochemical and immunological methods. 


\section{Washington State University Pullman, WA 99164-6340}

\section{A Comprehensive Approach to the Elucidation of Lignification at the Plasma Membrane/Cell Wall Interface}

N.G. Lewis, Institute of Biological Chemistry

$\$ 170,000$

Bimolecular phenoxy radical coupling processes account for 35 to $40 \%$ of all organic carbon circulating in the biosphere. Their products enable the successful development and survival of all life forms, i.e., via formation of metabolites such as plant lignins, lignans and suberins, algal cell wall polymers, fungal fruiting bodies, and insect cuticles (melanization). A major enigma in science is to define how these coupling reactions are controlled in vivo, since all in vitro studies have lacked specificity.

Various plant oxidases (laccases, peroxidases) and one-electron oxidants, were systematically studied for specificity of function. Using the monolignol, E-coniferyl alcohol, all catalyzed non-regio and non-stereospecific coupling. In the presence of a catalytically inactive $78 \mathrm{kDa}$ protein from Forsythia, however, coupling was both regio- and stereoselective, affording only $(+)$-pinoresinol. This discovery proves that additional factors are involved in phenoxy radical coupling other than non-specific oxidases. We have introduced the term dirigent (Latin: to align or guide) protein to describe this phenomenon. Additional work will seek to establish that an entire new class of such proteins exists.

Genes encoding the proteins affording pinoresinol, lariciresinol, and secoisolariciresinol have been cloned. These are essential for heartwood development in various conifers, for formation of the anticancer agent, podophyllotoxin (etoposide) and the cancer-preventing metabolites in high-fiber diets. A cell suspension culture from loblolly pine has also been used to establish that a nitrogen cycle is in effect in lignifying cells thereby enabling phenylpropanoid metabolism to continue unabated. The next phase of our studies will focus upon the regulation of the lignin, lignan, and suberin pathways.

\section{Washington State University Pullman, WA 99164-6340}

\section{Interdisciplinary Plant Biochemistry Research and Training Center N.G. Lewis, Institute of Biological Chemistry

The Plant Biochemistry Research and Training Center at Washington State University interfaces its biochemistry program with that of the major plant sciences. In its second year since inception, research training opportunities have been provided for 31 undergraduate internships, 12 graduate students (Ph.D. and M.S. degrees), and 4 postdoctorals. In addition to formal training and review processes, the Center has initiated a weekly Plant Biochemistry 
Seminar Series, a biannual Plant Biochemistry Summer Course (of 2 weeks duration, drawing students nationwide), various informal discussion sessions and evaluation processes, along with an annual review of the program by eminent plant biochemists.

A primary role of the Center is to conduct interdisciplinary research activities targeting problems that are not readily accessible with single principal investigator laboratories. The three areas of strategic importance targeted for these research studies include: regulation of biochemical pathways, plant cell wall formation, and signal transduction mechanisms. In order to solve the particular problem of choice, each trainee overlaps research activities between at least two laboratories, thereby obtaining the requisite research expertise to the problem at hand.

\section{Washington State University Pullman, WA 99164-6340}

\section{Enhancement of.Photoassimilate Utilization by Manipulation of ADP-glucose pyrophosphorylase \\ T.W. Okita, Institute of Biological Chemistry \\ $\$ 190,000$ (2 years)}

The goal of this project is to increase the conversion of photoassimilate into starch via manipulation of ADP-glucose pyrophosphorylase (AGP), a key regulatory enzyme of starch biosynthesis. Using a powerful mutagenesis-glgC complementation system we have identified many potential AGP mutants that are defective in catalysis and/or allosteric regulation. In the previous funding period, a down-regulatory mutant that required more than 45-fold greater levels of the activator, 3-phosphoglyceric acid (3-PGA), than the wildtype enzyme for maximum enzyme activity was identified. This phenotype was due to a single point mutation that resulted in the replacement of Pro52 by a Leu in the large subunit sequences. More recently, a second down-regulatory mutant was obtained that requires 6 - to 10-fold greater levels of the activator 3-PGA than normal. DNA sequence analysis showed that this defect in allosteric regulation was caused by the replacement of Asp413 by Ala. As Asp413 lies adjacent to a Lys residue that was previously identified by chemical modification studies to be important in the binding of 3-PGA, our results indicate that both the N-terminal and C-terminal ends of the large subunit are involved in 3-PGA binding. Studies on mutations in the small subunit are underway. Several of the small subunit mutants appear to be defective in binding of the substrates. The study of these AGP mutants will not only increase our knowledge on the structure-function relationships of this enzyme but will lead to novel strategies of increasing starch production and, in turn, increasing overall productivity for many crop plants. 


\section{Washington University \\ St. Louis, MO 63130}

\section{Plant Cell Wall Architecture}

Z.-H. Ye, Biology Department

$\$ 75,132$

We have further characterized the role of caffeoyl COA 3-O-methyltransferase (CCOAOMT) in lignification in dicotyledonous plants. Immunolocalization demonstrated that CCOAOMT is specifically localized in lignified tissues including phloem fibers, xylem cells and sclerified parenchyma cells from Forsythia, tobacco, tomato, soybean and alfalfa. To directly prove the roles of O-methyltransferases (OMTs) in lignification, we have been analyzing the effects of the antisense expression of OMTs on lignin content and composition in tobacco. While decreasing caffeicacid 3-O-methyltransferase (COMT) expression dramatically changed lignin composition, no change in lignin content was observed. No significant effect on lignin composition or content was observed as a result of decreasing CCOAOMT. These results indicate that decreasing one OMT expression has no effect on lignin content. We are currently trying to generate a transgenic tobacco line with simultaneous decrease of both COMT and CCOAOMT in the hope of finding a way to change lignin content.

As part of an effort to investigate the process of xylogenesis, we have isolated three novel hydrolase cDNAs. One is a RNase CDNA which is rapidly induced by mechanical wounding. The other two are RNase and cysteine protease cDNAs which were specifically induced during xylogenesis in cultured Zinnia cells and in Zinnia stems. In addition, the activity of a serine protease was markedly induced during xylogenesis. The results suggest that these hydrolases are involved in programmed cell death during xylogenesis. Isolation of these hydrolase genes provides molecular tools for the dissection of the, as yet poorly understood, process of programmed cell death during xylogenesis.

\section{University of Washington Seattle, WA 98195-1750}

\section{Genetics in Methylotrophic Bacteria}

M.E. Lidstrom, Department of Chemical Engineering

The purpose of this project is to study the transcriptional regulation of methanol oxidation (Mox) functions in the facultative methylotrophic bacterium, Methylobacterium extorquensAM1. Mox functions include a 14-gene operon (mxaFJGIRSACKLDH1H2B) containing genes required for production, assembly, and regulation of methanol dehydrogenase, a 5-gene cluster (pqqABCDE) and a 2-gene cluster (pqqFG) required for production of the methanol dehydrogenase prosthetic group, pyrroloquinoline quinone (PQQ), a two-gene cluster (mxdRS) and another gene (mxaW) of unknown function, and two additional sets of Mox regulatory genes, $m \times b D M$ and $m \times c Q E$. Sequence analysis of the regulatory genes suggests 
that they comprise three sets of sensor protein kinase/response regulator systems. We have studied the relationships between these systems using transcriptional fusions to $x y l E$ as a reporter gene. One of these regulatory systems, $M \times C Q E$, regulates expression of a second system, MxbMD. Expression of the third response regulator gene (mxaB) depends on expression of the mxaF operon, which requires all three sets of regulatory systems. Therefore, $m \times a B$ is autoregulated, but its expression also requires full expression of the other two systems. MxaB, along with $m \times b D M$, are required for expression of pqq genes, but only $\mathrm{mxbDM}$ are required for expression of $\mathrm{mxaW}$. These results suggest a hierarchy of regulatory systems that differentially control specific sets of Mox genes. The signals sensed by these three systems are unknown, but they are probably periplasmic, and the lack of identity between the putative periplasmic section of the sensor kinases suggests they may sense different signals.

\section{University of Wisconsin Madison, WI 53706-1381}

\section{Biochemical and Molecular Analysis of a Transmembrane Protein Kinase from Arabidopsis thaliana \\ A.B. Bleecker, Department of Botany \\ $\$ 95,000$}

Signal transduction involves perception of a signal by a receptor molecule and transduction of the signal to downstream components of the pathway through a series of phosphorylation events. Membrane-associated receptors with extracellular, ligand-binding domains and intracellular kinase domains are found in both animals and plants. We are trying to discover the role of TMK1, a transmembrane receptor-like protein kinase in Arabidopsis that resembles animal growth receptors. The main approaches are analysis of antisense TMK1 transgenic Arabidopsis, immunolocalization of TMK1, biochemical characterization of phosphorylation domains and interaction cloning. Antisense plants with reduced levels of TMK1 protein are reduced in size compared to wild type plants suggesting TMK1 is involved in growth regulation. TMK1 protein has been localized to the L1 layer of vegetative apices of developing plants. TMK1 cross-phosphorylates when expressed from bacterial expression vectors. The serine/threonine-rich $\mathrm{C}$-terminus is a substrate for phosphorylation, although truncated constructs lacking most of this region phosphorylate weakly. cDNAs that express weak interactors have been isolated in two-hybrid screens with both the leucine-rich region and kinase domains as bait. So far, no sequence identity for the cDNAs has been found. TMK1 interacts with a $2 A$ protein phosphatase (KAPP) isolated by Stone et al (U.M.-Columbia) in an interaction screen with RLK5, another receptor-like protein kinase. KAPP is a substrate for TMK1 in phosphorylation assays. Additional characterization of transgenic plants, including plants transformed with a TMK1 dominant-negative construct, and localization of TMK1 in a developmental series should contribute to our understanding of receptor-like protein kinases in plants. 


\section{University of Wisconsin Madison, WI 53706}

\section{Molecular Genetics of Ligninase Expression D. Cullen, Department of Bacteriology}

Lignin-degrading fungi play a key role in the carbon cycle, and numerous studies have demonstrated their potential in emerging technologies such as biomechanical pulping and the remediation of organopollutant-contaminated soils. These processes involve mechanism(s) which are poorly understood. Our long term objectives are to elucidate the basic genetics and physiology of the degradation of lignin and related aromatic compounds. Investigations are focused on the genomic organization and regulation of the genes encoding extracellular peroxidases and glyoxal oxidase of the white-rot fungus, Phanerochaete chrysosporium. Detailed physical and genetic maps are being constructed for all known structural genes and transposon-like elements. To establish the role of individual genes, peroxidase and glyoxal oxidase mRNA levels are being measured in woody material and in organopollutant-contaminated soils. Further, the importance of glyoxal oxidase in lignin degradation is being determined by gene disruption techniques. Finally, efficient heterologous expression systems are being developed for basic investigations of enzyme structure and function.

This research contributes to our understanding of the mechanism(s) by which fungi degrade lignin and related aromatic compounds. These studies also provide insight into lower. eukaryote genome organization and genetic regulation.

\section{University of Wisconsin Madison, WI 53706}

\section{Identification of the Primary Mechanism for Fungal Lignin Degradation K.E. Hammel, Department of Bacteriology $\$ 76,000$}

Microbial ligninolysis is an essential link in the terrestrial carbon cycle that is thought to be carried out almost entirely by wood- and litter-decomposing basidiomycetous fungi. These organisms produce a variety of extracellular oxidative agents, including hemoprotein lignin peroxidases (LiPs) that have the unusual ability to cleave the recalcitrant nonphenolic structures that predominate in lignin. However, many ligninolytic fungi appear to lack LiP activity, and the mechanisms by which lignin is initially degraded in wood remain unclear. Work on this project has shown that one LiP-negative fungus, Ceriporiopsis subvermispora, nevertheless cleaves nonphenolic lignin structures efficiently. Nuclear magnetic resonance spectrometry of the high molecular weight products that the fungus produced from a new polyethylene glycol-linked ${ }^{13} \mathrm{C}$-labeled arylglycerol-2-aryl ether lignin model compound showed that this major lignin structure was cleaved between $\mathrm{C} 1$ and $\mathrm{C} 2$ of its propyl side chain. 
Chromatographic analysis of the low molecularweight products that the fungus produced from a polyethylene glycol-linked ${ }^{14} \mathrm{C}$-labeled arylglycerol-2-aryl ether lignin model compound indicated that aryl ether cleavage probably occurred as well during degradation. These results suggest that $C$. subvermispora produces a ligninolytic agent other than LiP and that this agent oxidizes lignin structures either by ionizing them to cation radicals or via oxyradical addition to an aromatic ring.

\section{University of Wisconsin Madison, WI 53706}

\section{Organization of the $\mathbf{R}$ Chromosome in Maize J. Kermicle, Laboratory of Genetics}

The maize $r$ gene product activates expression of structural genes in the 3-hydroxy anthocyanin pathway. Different $r$ genes (individual alleles) confer pigmentation on different plant parts. In some strains multiple $r$ genes are clustered on chromosome 10 forming complexes. In certain heterozygotes the level of $r$ action is heritably silenced, i.e., paramutated. We want to know how the potential of one $r$ complex to silence genes in the homologous chromosome is organized and how the change is brought about. Is silencing facilitated when the inciting and responding genes are in the same rather than in homologous chromosomes? When two responding genes and one inciting gene are present in trisomic plants, are the responding genes affected coordinately or independently? When during plant development does silencing occur? What is the relation of heritable silencing, which occurs during plant development, to a somatic inhibition of $r$ pigmentation observed in certain $F_{1}$ kernel genotypes? We also want to know what other loci are necessary for $r$ silencing to occur. Our general strategy is to dissect silencing genetically, then characterize selected aspects molecularly.

\section{University of Wisconsin Madison, WI 53706-1569}

\section{The Biochemistry, Bioenergetics and Physiology of the CO-dependent Growth of Rhodospirillum rubrum P.W. Ludden, Department of Biochemistry

Rhodospirillum rubrum is a purple, non-sulfur photosynthetic bacterium that is capable of growth with carbon monoxide as the carbon and energy source. CO-dependent growth requires Carbon Monoxide Dehydrogenase (CODH), a Ni-Fe-S-containing enzyme that oxidizes $\mathrm{CO}$ to $\mathrm{CO}_{2}$, and a $\mathrm{CO}$-induced hydrogenase. The $R$. rubrum $\mathrm{CODH}$ is much simpler than the enzymes from acetogens and methanogens which function as acetyl-CoA synthetases in those organisms. The $R$. rubrum enzyme contains the 4Fe4S B cluster and 
the 4Fe4S-Ni C cluster. Recently a model for the structure of the $\mathrm{C}$ cluster has been proposed in our laboratory, and a goal of this project will be to confirm that structure and to identify the ligands for the $B$ and $C$ clusters in the enzyme. Site-specific mutations which result in accumulation of native but inactive $\mathrm{CODH}$ have been obtained, and the mutant forms of CODH from these organisms have been purified. Characterization of these mutant forms of $\mathrm{CODH}$ will be a goal of this project. Crystals of CODH have been obtained; and, in collaboration with others, the determination of the crystal structure of the enzyme will be a goal of this project. The $\operatorname{coo} \mathrm{C}, \mathrm{T}$, and $\mathrm{J}$ gene products have been identified as playing a role in the processing of nickel for $\mathrm{CODH}$. Characterization of the $\mathrm{COOC}_{1} \mathrm{~T}$, and $\mathrm{J}$ gene products will be a goal of this project, and their roles in the nickel processing will be investigated.

\section{University of Wisconsin Madison, WI 53706}

\section{Feedback Regulation of Photosynthetic Processes \\ T.D. Sharkey, Department of Botany}

Photosynthetic carbon accumulation requires chloroplast and cytosolic reactions. In this project, the interaction between photosynthesis and cytosolic sucrose synthesis are studied with biochemical and gas exchange techniques using naturally occurring mutant plants and transgenic plants. Naturally occurring mutants lacking all cytosolic fructose-bisphosphatase, considered essential for sucrose synthesis, have been studied in detail. We propose that carbon is exported from the chloroplast as triose phosphate during the day but as hexose phosphate at night. The hexose transporter must be highly regulated. It must be off during the day, forcing carbon through cytosolic FBPase, and active at night. The export of hexose phosphate at night bypasses the cytosolic FBPase allowing the FBPase-lacking plant to survive. The effects of increasing the capacity for sucrose synthesis on yield are studied using plants expressing sucrose phosphate synthase (SPS) on either a rubisco small subunit promoter or the cauliflower mosaic virus $35 S$ promoter. The SSU promoter increases SPS in the leaf 6 fold and in the fruit 2 fold. The $35 S$ promoter increases SPS in the leaf 2 fold and in the roots 2 to 10 fold. We are manipulating the SPS level in the leaf to test whether there is an optimum SPS level for yield or whether SPS in other organs affects yield. One tomato line routinely exhibits substantially increased fruit yield as do potatoes with excess SPS. This work will contribute to our understanding of how photosynthesis works and to increased plant yield through biotechnology. 


\section{University of Wisconsin Madison, WI 53706}

\section{Molecular Mechanism of Energy Transduction by Plant Membrane Proteins M.R. Sussman, Department of Horticulture \\ $\$ 106,000$}

Proton pumps are membrane-bound proteins that act as biological energy transducers. The plasma membrane of plants and fungi contains an ATP-coupled proton pump ( $\mathrm{H}^{+}$-ATPase) that converts chemical into electrical energy, and provides the driving force to catalyze the uptake and accumulation of solutes. The plant/fungal plasma membrane $\mathrm{H}^{+}$-ATPase contains a single polypeptide of $\mathrm{Mr}=100,000$. Its simple polypeptide structure makes this enzyme an attractive candidate for studying the molecular basis of energy transduction. A major aim of this project is to identify aspects of the enzymes' primary structure that are essential for converting chemical into electrical energy. DNA cloning and sequencing techniques are being used to obtain the complete amino acid sequence for twelve $\mathrm{H}^{+}$-ATPase structural genes present in Arabidopsis thaliana, a model higher plant with a small genome and a rapid generation time. Mutant $A$. thaliana plants in which each of these ATPase genes is disrupted are being isolated. The phenotypes of these plants will help to determine the biological role of each pump isoform in situ. Expression of the genes is being studied using Northern blots and 'epitope-tagging' to produce isoform-specific polypeptide probes. Overall, these studies are essential for testing hypotheses concerning the biological role of ion pumps and the molecular mechanism of protein-mediated energy transduction in plants.

\section{University of Wisconsin Madison, WI 53706}

\section{Analysis of Structural Domains Required for Phytochrome Function by in vitro Mutagenesis \\ R.D. Vierstra, Department of Horticulture

Phytochrome is a red/far-red photoreversible photoreceptor that has a central role in light-regulated plant development. To determine how phytochrome functions at the molecular level, we have exploited a biological assay for active chromoproteins that involves the expression of chimeric phytochrome genes in transgenic tobacco. Such ectopic overexpression induces a striking "light exaggerated" phenotype that can be used as an in vivo assay of receptor function. The goal of the project is to combine this transgenic system with in vitro mutagenesis to identify phytochrome domains important to synthesis, dimerization, chromophore attachment, Pr/Pfr phototransformation, Pfr-enhanced degradation, and biological activity. Preliminary mapping has localized an important region near the $\mathrm{N}$-terminus of phytochrome $\mathrm{A}$ (residues 1-70) that is essential for function and spectral integrity. Within this domain, we recently discovered two distinct domains, one required for the structural integrity and biological activity of the chromoprotein, and a second, serine-rich 
domain that modulates phytochrome activity. Removal of or substitutions within this second domain generate a hyperactive photoreceptor with 10-50 greater sensitivity to far-red light. Biochemical and genetic approaches are now in progress to determine the mechanism(s) that underlie this hyperactivity since it may reflect a novel post-translational step that attenuates phytochrome A function. Completion of this work will represent an important step in the identification of domains essential to the proper assembly and function of this essential photoreceptor and should lead to an understanding of its regulation and mode of action.

\section{Worcester Foundation for Biomedical Research Shrewsbury, MA 01545}

\section{Novel Biomaterials: Genetically Engineered Pores H. Bayley}

A collection of nanometer-scale pores is being constructed by remodelling $\alpha$-hemolysin ( $\alpha \mathrm{HL}$ ), a protein secreted by the bacterium Staphylococcus aureus. The single polypeptide chain of 293 amino acids self assembles to form heptameric pores in membranes $\sim 11 \AA$ in internal diameter. By analyzing the properties of mutant and chemically modified $\alpha H L s$, a working model for assembly has been devised, which is generally compatible with the newly available three-dimensional structure of the pore. Monomeric $\alpha H L$ first binds to lipid bilayers and then aggregates to form a heptameric prepore complex. Finally, the open pore is formed when subunits in the complex undergo a cooperative conformational change, involving both the central glycine-rich loop and the N-terminus of the polypeptide. The central loop lines a section of the transmembrane channel in the fully assembled pore. Continuing studies of assembly and function are allowing point mutagenesis, combinatorial mutagenesis and targeted chemical modification to be used to create pores with new properties. For example, triggers and switches have been built into $\alpha H L$ to gain control over the opening and closing of the pores. Inputs that actuate the reengineering molecules can be biochemical in nature (activation by specific proteases), chemical (modulation of activity by covalent and noncovalent interactions with small molecules) and physical (activation by light). The new pores will be used to confer novel permeability properties upon materials such as thin films, which might then be used as components of energy conversion and storage devices, selective electrodes, electronic devices, and ultrafilters.

*Funded collaboratively with Division of Materials Sciences (DOE). 


\section{Xavier University of Louisiana New Orleans, LA 70125}

\section{Molecular Characterization of Bacterial Respiration on Minerals} R. Blake II, College of Pharmacy

Aerobic respiration on reduced iron is a principal metabolic activity exhibited by certain chemolithotrophic bacteria that inhabit ore-bearing geological formations exposed to the atmosphere. Each phylogenetically distinct group of iron-oxidizing bacteria expresses one or more unique acid-stable, redox-active biomolecules in conspicuous quantities during aerobic respiration on iron. Structural and functional studies continue on two such novel biomolecules that have been purified to electrophoretic homogeneity, rusticyanin from Thiobacillus ferrooxidans and cytochrome ${ }_{579}$ from Leptospirillum ferrooxidans. The aim of these studies is to determine the role of each protein in the iron respiratory chain of its respective organism. Other redox-active components present in cell-free extracts of iron-oxidizing organisms continue to be sought, isolated, and investigated with regard to their roles in the same respiratory chains. Another aim is to investigate the mechanisms, consequences, and principal features of bacterial adherence to insoluble metal sulfides. These studies exploit electrical impedance, surface tension, contact angle, surface area, and other measurements commonly employed to characterize colloidal particles. It is anticipated that this project will provide useful information toward manipulating $T$. ferrooxidans and related organisms for commercial use.

\section{Xavier University of Louisiana New Orleans, LA 70125}

\section{Biochemistry of Dissimilatory Sulfur Oxidation}

R. Blake II, College of Pharmacy

Dissimilatory sulfur-oxidizing bacteria obtain all of their energy for metabolism from the aerobic oxidation of reduced inorganic sulfur compounds. Despite the environmental and economic importance of these organisms, there is still much uncertainty regarding the actual metabolic pathways and the stoichiometries of these bacterial oxidation reactions. This project has two major experimental goals: (i) to quantify the adherence of sulfur-oxidizing bacteria to their insoluble elemental substrate; and (ii) to define the substrate oxidation pathways, the electron transport mechanisms, and the modes of energy conservation employed by various species of the thiobacilli. Studies on the interaction between bacteria and insoluble sulfur exploit field flow fractionation, laser Doppler velocimetry, electrical impedance, static and dynamic light scattering, and other measurements commonly employed to characterize colloidal particles. Studies on the latter goal currently focus on the enzymology of polythionate degradation in those thiobacilli that accumulate polythionates in the culture medium. Efforts to isolate and characterize trithionate hydrolase, thiosulfate 
dehydrogenase, and tetrathionate hydrolase from cell-free extracts of Thiobacillus neapolitanus and $T$. intermedius are in progress. It is anticipated that this information will be of value in the eventual manipulation of the thiobacilli and related organisms to benefit both the environment and the mining industry.

\section{Yale University}

New Haven, CT 06520-8104

\section{Molecular Genetics of the R-r Complex of Maize \\ S. Dellaporta, Department of Biology}

$\$ 92,000$

The R-r complex of maize is a cluster of genes on the long arm of chromosome 10. Each member of the complex encodes highly related transcription factors required for anthocyanin biosynthesis in seed or plant parts. This complex exhibits meiotic instability, with loss of seed or plant color capacity associated with unequal exchanges or other mechanisms not associated with crossing over. Past studies have focused on instability associated with unequal exchanges and the molecular organization of the complex. This year our efforts have been to understand the basis of meiotic mutations in the R-p gene of the R-r complex of maize. The majority of mutations in the $R-p$ gene were shown to be insertions of a novel transposable element, termed PIF (P Instability Factor). PIF differs from other maize elements in that it shows a strong target site preference -- four out of five insertions were found in the exact same target site in the $R-p$ gene. These studies complete our analysis of meiotic instability of the $R$ complex. Our future studies will focus on the origins of $R$ complexity by examining this region in races of maize and sister taxa.

\section{Yale University}

New Haven, CT 06511-7444

\section{Spatial Regulation of $\mathrm{C}_{4}$ Genes in $\mathrm{C}_{3}, \mathrm{C} 4$, and $\mathrm{C} 3 / \mathrm{C} 4$ Intermediate Flaveria Species \\ T. Nelson, Biology Department \\ $\$ 129,634$}

The dicot genus Flaveria (Asteraceae) includes species utilizing C3, C4, and C3/C4 intermediate schemes of carbon fixation. In the efficient $\mathrm{C} 4$ scheme, neighboring photosynthetic bundle sheath (BS) and mesophyll (M) cells cooperate for carbon fixation. $\mathrm{CO}_{2}$ is fixed initially in C4 acids in M cells, then further metabolized in BS cells, via a pathway that relies on expression of the genes for pathway enzymes in $M$ - or BS-specific patterns. In less efficient $\mathrm{C} 3$ species, $M$ cells independently fix $\mathrm{CO}_{2}$ in a $\mathrm{C} 3$ compound, and the $\mathrm{BS}$ is not generally photosynthetic. C3/C4 intermediate species exhibit anatomical and biochemical characteristics between the $\mathrm{C} 4$ and $\mathrm{C} 3$ extremes and may represent evolutionary steps between $\mathrm{C} 3$ and $\mathrm{C} 4$ forms. In both $\mathrm{C} 3$ and $\mathrm{C} 3 / \mathrm{C} 4$ species, $\mathrm{C} 4$ pathway genes are present, 
but used in different spatial patterns. The variety of Flaveria species provides an opportunity to compare the structure and regulation of $\mathrm{C} 4$ pathway genes in closely related $\mathrm{C} 3$ and $\mathrm{C} 3 / \mathrm{C} 4$ species. We have isolated and characterized example genes encoding malic enzyme (ME), ribulose bisphosphate carboxylase (rbcS), and malate dehydrogenase (MDH) from C3 and C4 species of Flaveria. Our ongoing work includes the characterization of spatial and temporal expression patterns of these genes in $\mathrm{C} 3, \mathrm{C} 4$ and $\mathrm{C} 3 / \mathrm{C} 4$ species and the expression of ME-, rbcS-, and MDH-reporter gene fusions in leaf cells of C3, C4 and C3/C4 species. Our work to date suggests that the observed cell-specific patterns are the consequence of both transcriptional and posttranscriptional regulation.

\section{Yale University}

\section{New Haven, CT 06520-8103}

\section{Role of Phenolics in the Adaptation of Rhizobia to the Rhizosphere and Soil D. Parke and L.N. Ornston, Department of Biology}

$$
\$ 136,520 \text { (FY } 95 \text { funds/18 months) }
$$

The protocatechuate branch of the $\beta$-ketoadipate pathway is central to the catabolism of 4-hydroxybenzoate and numerous other phenolic compounds. The pathway is widely distributed among members of the bacterial group the Rhizobiaceae. Genetic elements underlying 4-hydroxybenzoate dissimilation in Agrobacterium tumefaciens have been characterized. Eleven pca (protocatechuate) and pob (4-hydroxybenzoate) genes are organized in a compact supraoperon. Evolutionary divergence of $A$. tumefaciens from phylogenetically distant bacteria is reflected in the layout of the pob-pca supraoperon as well as in novel regulatory motifs that govern expression of the genes. Regulatory control over the pca-pob genes is exerted by three regulators, which belong to three different classes of regulatory proteins. Tight clustering of the pca-pob regulatory genes is taken to an extreme with an unusual 3' overlap of two genes. Grouped together with Agrobacterium in the family Rhizobiaceae are rhizobial species which form nitrogen-fixing nodules on roots in a symbiotic partnership with plants. At least some of the distinctive pca-pob regulatory features found in Agrobacterium are shared with its rhizobial relatives.

Flavonoid compounds serve as inducers of nodulation genes in rhizobia. Certain flavonoid inducers can be broken down by rhizobia into monocyclic derivatives, which include protocatechuate or its metabolic precursors. Further studies will explore the role of flavonoids and other phenolic compounds in rhizobial growth and symbiosis. Analysis of flavonoid dissimilatory pathways and their regulatory controls will use HPLC augmented by plasmid constructions that serve as indicators for phenolic degradation via the $\beta$-ketoadipate pathway. 


\section{Yale University}

New Haven, CT 06510

\section{Calcium Mapping and Signalling in Yeast \\ C.L. Slayman, Department of Cellular and Molecular Physiology}

$\$ 121,000$

Current work represents a fusion of previous studies from this laboratory, on transport mechanisms in the vacuolar membrane (tonoplast) of Saccharomyces, with new possibilities for analysis of intracellular signalling mechanisms which have arisen from completion of the genome sequencing project.

The predominant yeast vacuolar cation channel (YVC1, Proc. Natl. Acad. Sci. 87:7824,1990) now appears to be an intracellular calcium release channel $\left(I C R_{\mathrm{Ca}}\right)$, perhaps the prototype for such channels in all plants and fungi. Experiments on functional characterization of the channel by means of isolated-patch and whole-vacuole recording with patch electrodes are continuing, particularly with respect to regulatory factors: cytosolic calcium concentration, tonoplast voltage, and cytoplasmic redox elements. A previous project to isolate and characterize the channel protein by standard biochemical techniques has given way to fluorescent immunochemical localization based on the amino acid structures implied by gene sequences for the five $\mathrm{Ca}^{++}$-channel-like molecules in yeast.

Despite severe instrumentational limitations, we are also starting a project to map the intracellular distribution of calcium under various conditions and to test quantitatively specific signalling hypotheses by examining spatial and temporal changes in $\mathrm{Ca}^{++}$distribution upon deletion/disruption of known calcium-sensitive or calcium-dependent proteins. This project is based on the use of several different calcium-indicators (in conjunction with high-resolution full-field fluorescence microscopy) and upon the use of enlarged yeast cells (stable octaploids) to enhance spatial resolution.

\section{Yale University}

New Haven, CT 06520-8114

\section{Transfer RNA Involvement in Chlorophyll Biosynthesis}

D. Söll, Department of Molecular Biophysics and Biochemistry

$\$ 109,000$ (FY 95 funds/20.5 months)

Chlorophyll is the major pigment in photosynthesis. One molecule of chlorophyll is synthesized from eight molecules of 5-aminolevulinic acid (ALA), the universal precursor of porphyrins. The initial metabolite for the $C 5$ pathway is Glu-tRNA which in the presence of NADPH is converted by the action of an unusual enzyme, Glu-tRNA reductase (GluTR), to glutamate-1-semialdehyde (GSA) with the concomitant release of tRNA. Glu-tRNA is a dual-function molecule; it provides glutamate for protein synthesis and GSA for porphyrin 
synthesis. The regulation of the flow of Glu-tRNA into the different pathways may be determined by the relative concentrations of EF-T.u and GluTR, two proteins which compete in binding Glu-tRNA. In the second step of the pathway an aminotransferase (GSA-amino-1,2-mutase, GSA-AM) converts GSA to ALA.

To further examine ALA formation in plants, we have isolated genes of Arabidopsis thaliana that encode the enzymes of the C5 pathway, via functional complementation of mutations in the corresponding genes of $E$. coli. There are two genes for each of the two enzymes of the C5 pathway. The HEMA and HEMA2 gene products (GluTR enzymes) show $85 \%$ amino acid identity, but are expressed very differently. In contrast, the GSA1 and GSA2 genes are much more related. It was observed that the HEMA2 gene is preferentially expressed in the non-chlorophyll containing tissue, while the HEMA1 gene is expressed in leaves. Currently experiments are underway with GUS reporter gene fusions to determine the DNA regions important for gene expression. To examine the effect of the suppression of the HEMA and GSA genes, transgenic Arabidopsis plants which express antisense mRNA for these two enzymes were generated. These plants show varying degrees of chlorophyll deficiency, suggesting that the formation of ALA by HEMA and GSA genes is an obligatory step in the biosynthesis of chlorophyll. Transgenics that express an antisense HEMA gene show not only lesser amounts of chlorophyll (20\% to $75 \%)$, but also ALA when compared to the untransformed plants. 


\section{PROJECT CATEGORIZATION}

What follows is a grouping of Energy Biosciences projects into a few major topic areas that characterize the overall objectives of the program. Projects that overlap different categories are marked by an asterisk $\left({ }^{*}\right)$, and are listed in both categories. Each project has been assigned a number which identifies the abstract found within this report.

\section{PHOTOSYNTHESIS}

The conversion of solar energy into chemical energy by photosynthesis is the energetic underpinning for life on earth that ultimately results in renewable resources. Important research topics include dissecting the numerous aspects of photosynthesis such as carbon fixation, oxygen evolution, photorespiration, photophosphorylation, structures of photosynthetic elements and other topics relating to photosynthesis under natural conditions. A great diversity of techniques ranging from ultrafast laser spectroscopy to site directed mutagenesis are being utilized from the molecular to the whole plant level. The intent is to understand the most critical biological energy conversion process upon which most life depends.

Abs. 5 Antenna Organization and Regulation in Green Photosynthetic Bacteria R.E. Blankenship, Arizona State University

Abs. 6 Chlorophyll-Binding and Biogenesis of Photosystem II W. Vermaas, Arizona State University

Abs. 18 Molecular Bases of Photoadaptation in Unicellular, Eucaryotic Algae P.G. Falkowski and J. LaRoche, Brookhaven National Laboratory

Abs. 19 Regulation of Energy Conversion in Photosynthesis

G. Hind, Brookhaven National Laboratory

Abs. 50 Molecular Genetic Analysis of Biophotolytic Hydrogen Production in Green Algae

L. Mets, University of Chicago

Abs. 64 Molecular, Genetic and Physiological Analysis of Photoinhibition and Photosynthetic Performance

J.E. Boynton, N.W. Gillham and C.B. Osmond, Duke University 
152 / Division of Energy Biosciences - Summaries of FY 1996 Activities

Abs. 82 Violaxanthin De-epoxidase: Biogenesis and Structure

H.Y. Yamamoto, University of Hawaii

Abs. 87 Integrative Photosynthesis Research

C.A. Wraight, D.R. Bush, J.M. Cheeseman, A.R. Crofts, P.G. Debrunner,

E.H. DeLucia, Govindjee, D.R. Ort, A.R. Portis, J. Whitmarsh, R.E. Zielinski, University of Illinois

Abs. 92 Transport of lons Across the Inner Envelope Membrane of Chloroplasts

R.E. McCarty, Johns Hopkins University

Abs. 93 Macromolecular Scaffolds for Energy Transfers

D.C. Youvan, KAIROS Scientific Inc.

Abs. 95 Mechanism and Significance of Post-Translational Modifications in the Large Subunit of Ribulose-Bisphosphate Carboxylase/Oxygenase

R.L. Houtz, University of Kentucky

Abs. 99 Transferring Photosynthesis Genes to New Cellular Environments J.E. Hearst, Lawrence Berkeley National Laboratory

Abs. 100 Mechanism of Water Splitting and Oxygen Evolution in Photosynthesis M.P. Klein and V.K. Yachandra, Lawrence Berkeley National Laboratory

Abs. 101 Photosynthetic Pigment Proteins and Photosynthetic Light Reactions K. Sauer, Lawrence Berkeley National Laboratory

Abs. 140 The Water-Splitting Apparatus of Photosynthesis M. Seibert, National Renewable Energy Laboratơry

Abs. 155 Photosynthetic Electron Transport in Genetically Altered Chloroplasts R.T. Sayre, Ohio State University

Abs. 156 Regulation of Alternative $\mathrm{CO}_{2}$ Fixation Pathways in Procaryotic and Eucaryotic Photosynthetic Organisms

F.R. Tabita, Ohio State University

Abs. 164 Light-Energy Transduction in Green Sulfur Bacteria D.A. Bryant, Pennsylvania State University

Abs. 172 Membrane-Attached Electron Carriers in Photosynthesis and Respiration F. Daldal, University of Pennsylvania 
Abs. 177 A Genetic Analysis of the Lumenal Proteins of the Photosystem $\| \mathrm{O}_{2}-$ evolving Complex in Cyanobacteria

L.A. Sherman, Purdue University

Abs. 190 Molecular Mechanisms Controlling Proton Pumping by Bacteriorhodopsin R.K. Crouch, Medical University of South Carolina

Abs. 197 Ferredoxin-Linked Chloroplast Enzymes

D.B. Knaff, Texas Tech University

Abs. 201* Structural Domains in NADPH: Protochlorophyllide Oxidoreductases Involved in Catalysis and Substrate Binding

M.P. Timko, University of Virginia

Abs. 215 Feedback Regulation of Photosynthetic Processes

T.D. Sharkey, University of Wisconsin

Abs. 222 Spatial Regulation of C4 Genes in C3, C4, and C3/C4 Intermediate Flaveria Species

T. Nelson, Yale University

Abs. 225 Transfer RNA Involvement in Chlorophyll Biosynthesis

D. Söll, Yale University

\section{MEMBRANES OR ION TRANSPORT}

Membranes provide a selective barrier around a cell as well as delineate the organelles within the cell. Many critical metabolic processes are membrane "bound" or occur across membranes including key steps in the energy transducing processes of photosynthesis and respiration. Knowledge about the structure and properties of different membrane systems is essential to an understanding of how plants use the available photosynthetically derived energy and how organisms absorb, transport and utilize mineral ion nutrients in sustaining their growth, development and other synthetic activities.

Abs. 4 Phytoremediation of Metal-Polluted Soils: Mechanisms of Heavy Metal Absorption, Translocation, Accumulation and Tolerance in Plants L.V. Kochian, USDA - Ithaca, NY

Abs. 12 Molecular Characterization of the Role of a Calcium Channel in Plant Development K.S. Schumaker, University of Arizona 
154 / Division of Energy Blosclences - Summaries of FY 1996 Activities

Abs. $15^{*} \quad$ Osmoregulation in Methanogens

M.F. Roberts, Boston College

Abs. 35 Protein Translocation and Assembly in Chloroplasts

S.M. Theg, University of California - Davis

Abs. 36* Vacuole Biogenesis in Differentiating Plant Cells

T.A. Wilkins, University of California - Davis

Abs. 39 Molecular Structure, Function and Physiology of $\mathrm{K}^{+}$Uptake Channels in Plants

J.I. Schroeder, University of California - San Diego

Abs. 44 Regulation of Vacuolar pH in Citrus limon

L. Taiz, University of California - Santa Cruz

Abs. 59 Effects of Freezing and Cold Acclimation on the Plasma Membrane of Isolated Cereal Protoplasts

P.L. Steponkus, Cornell University

Abs. 105 Structure and Regulation of Calcium-Pumping ATPases in Plants

H. Sze, University of Maryland

Abs. 117 Molecular Mechanisms of Trafficking in the Plant Cell

N.V. Raikhel, Michigan State University. DOE Plant Research Laboratory

Abs. 174 Structure-Function Analysis of Vacuolar $\mathrm{H}^{+}$-Pyrophosphatase

P.A. Rea, University of Pennsylvania

Abs. 183 Molecular Analysis of a Thylakoid $\mathrm{K}^{+}$Channel

C. Chin, Rutgers University

Abs. 188 Membrane Targeting of P-type ATPases in Plant Cells

J. Harper, The Scripps Research Institute

Abs. 195* Molecular Mechanism of Light-Regulated Protein Transport Across the Nuclear Envelope

A.G. von Arnim, University of Tennessee

Abs. 216 Molecular Mechanism of Energy Transduction by Plant Membrane Proteins M.R. Sussman, University of Wịsconsin

Abs. 224 Calcium Mapping and Signalling in Yeast

C.L. Slayman, Yale University 


\section{PLANT METABOLISM AND BIOSYNTHESES}

One of the greatest resources plants have to offer is the ability to synthesize a massive variety of products that provide food, fibers, structural components, pharmaceuticals and numerous materials and chemicals for other uses. In order to have greater ability to use plants as a resource in the rapidly growing biotechnology industry, it is essential to build the base of understanding of not only the metabolic capabilities of plants, but how the various pathways are regulated. The availability of newer techniques for chemical analyses, in addition to the formidable tools of molecular genetics, have made it possible to probe questions that previously were almost intractable. Aspects of research on the major storage products, carbohydrates and lipids are listed as subheadings of this category.

Abs. $21 \quad \delta$-Aminolevulinate Biosynthesis in Oxygenic Prokaryotes

S. Beale, Brown University

Abs. 32 Plant Physiological Aspects of Silicon

E. Epstein, T.W-M. Fan, W.K. Silk, and R.M. Higashi, University of California - Davis

Abs. 52 The Magnesium Chelation Step in Chlorophyll Biosynthesis

J.D. Weinstein, Clemson University

Abs. 67 Gene-Enzyme Relationships of Aromatic Amino Acid Biosynthesis in Higher Plants

R.A. Jensen, University of Florida

Abs. 78 Nitrogen Control of Chloroplast Development and Differentiation G.W. Schmidt, University of Georgia

Abs. 143 Asparagine Synthetase Gene Regulation and Plant Nitrogen Metabolism

G. Coruzzi, New York University

Abs. 201* Structural Domains in NADPH: Protochlorophyllide Oxidoreductases Involved in Catalysis and Substrate Binding

M.P. Timko, University of Virginia

Abs. 203 Regulation of Terpene Metabolism

R. Croteau, Washington State University

Abs. 206 Interdisciplinary Plant Biochemistry Research and Training Center N.G. Lewis, Washington State University 


\section{3a. CARBOHYDRATE METABOLISM}

Carbohydrates comprise a dominant component of the storage products and biomass of plants mostly in the forms of starch and the polymers of the cell wall. In addition to supplying the critical building blocks for plant metabolism and plant structure, carbohydrates are increasingly identified as important components of glycoproteins, glycolipids and polysaccharides, which have important regulatory functions in plant growth and development.

Abs. 3 Regulation of Sucrose-Phosphate Synthase and Other Cytosolic Proteins by Reversible Protein Phosphorylation

S.C. Huber, USDA - North Carolina State University

Abs. 7 Polyol Functions in Stress Protection of Photosynthesis

H.J. Bohnert and R.G. Jensen, University of Arizona

Abs. 65 Role of Sucrose in Modulating Stomatal Aperture

W.H. Outlaw, Jr., Florida State University

Abs. 126 Structure-Function Relationships of ADP-glucose Pyrophosphorylase:

Manipulation of the Plant Gene for Increased Production of Starch in Plants J. Preiss, Michigan State University

Abs. 148 Control of Sugar Transport and Metabolism in Zymomonas mobilis T. Conway, Ohio State University

Abs. 168 Molecular-Genetic Analysis of Maize Starch Branching Enzyme Isoforms M. Guiltinan, Pennsylvania State University

Abs. 179* A Molecular-Genetic Approach to Studying Source-Sink Interactions in Arabidopsis thaliana

S.I. Gibson, Rice University

Abs. 204* Carbon Metabolism in Symbiotic Nitrogen Fixation

M.L. Kahn, Washington State University.

Abs. 207* Enhancement of Photoassimilate Utilization by Manipulation of ADP-glucose pyrophosphorylase

T.W. Okita, Washington State University 


\section{3b. LIPID METABOLISM}

Within the past decade many of the enzymes involved in the biosynthesis of a variety of fatty acids and oils in plants have been identified, and commercial research is actively undenway to genetically engineer plants to produce higher-value plant oils for food and industrial uses. However, many unanswered questions remain on how the biochemical pathways regulate which fatty acids are synthesized and how they are partitioned among the biosynthesis of membrane components, storage oils and other lipid constituents of the plant cell.

Abs. 20 Modification of Plant Lipids

J. Shanklin, Brookhaven National Laboratory

Abs. 45* Production of Lipophilic Materials from Plants C.R. Somerville, Carnegie Institution of Washington

Abs. 96 Studies of a Novel Pathway for Biosynthesis of Straight and Branched, Odd and Even Length, Medium-Chain Fatty Acids in Plants

G.J. Wagner, University of Kentucky

Abs. 124 Control of Triacylglycerol Biosynthesis in Plants

J. Ohlrogge, Michigan State University

Abs. 125 A National Cooperative for Genetic Engineering of Plant Lipids

J. Ohlrogge, Michigan State University

Abs. 151* Biosynthesis of Hydrocarbons

P.E. Kolattukudy, Ohio State University

Abs. 202 Membrane Function in Lipid Mutants of Arabidopsis

J. Browse, Washington State University

\section{PLANT GROWTH AND DEVELOPMENT}

Clearly, the productivity of plants for maximal biomass requires knowledge about the nature of how cells and tissues expand and grow, as well as differentiate into the different organs of the plant. Over the last decade molecular genetic approaches have completely revitalized the manner in which plant growth and development are studied and are leading to significant advances in our understanding of these processes. 
158 / Division of Energy Biosciences - Summaries of FY 1996 Activities

Abs. 2 Metabolic Regulation of the Plant Hormone Indole-3-acetic Acid J.D. Cohen and J.P. Slovin, USDA - Beltsville, MD

Abs. 11 Role of Zein Proteins in Structure and Assembly of Protein Bodies and Endosperm Texture

B.A. Larkins, University of Arizona

Abs. 22 Molecular and Genetic Analysis of LEAFY, a Gene Controlling 'Floral Induction and Flower Development in Arabidopsis thaliana

E. Meyerowitz, California Institute of Technology

Abs. 23 The Molecular Genetics of Ligule Induction

M. Freeling, University of California - Berkeley

Abs. 27 Phytochrome from Green Plants: Properties and Biological Function P.H. Quail, University of California - Berkeley

Abs. 29 Analysis of Genes Essential for Floral Development in Arabidopsis P. Zambryski, University of California - Berkeley

Abs. 33 Regulation of Embryonic Development in Higher Plants J.J. Harada, University of California - Davis

Abs. $34^{*} \quad$ Cellular and Molecular Characterization of Vascular Plasmodesmata W.J. Lucas, University of California - Davis

Abs. $36^{\star} \quad$ Vacuole Biogenesis in Differentiating Plant Cells T.A. Wilkins, University of California - Davis

Abs. 38 Structure, Biosynthesis and Role of Complex Protein-Bound Glycans M.J. Chrispeels, University of California - San Diego

Abs. 41 The Gibberellin $A_{20} 3$-hydroxylase: Isolation of the Enzyme and Its Molecular Biology

B.O. Phinney and J. MacMillan, University of California - Los Angeles

Abs. $42^{*} \quad$ Sensory Transduction of the $\mathrm{CO}_{2}$ Response of Guard Cells

E. Zeiger, University of California - Los Angeles

Abs. 51 Analysis of Cell-Cell Interactions during Arabidopsis Reproduction

D. Preuss, University of Chicago 
Abs. 56 Cytoplasmic Male Sterility and Mitochondrial Function During Microsporogenesis M.R. Hanson, Cornell University

Abs. 57 Signal Transduction in the Pollen-Stigma Interactions of Brassica J.B. Nasrallah and M.E. Nasrallah, Cornell University

Abs. 63 Metabolic Mechanisms of Plant Growth at Low Water Potentials J.S. Boyer, University of Delaware

Abs. $74 \quad$ Genetic Analysis of Embryo Dormancy

G.A. Galau, University of Georgia

Abs. 85 Regulation of Cell Division in Higher Plants T. Jacobs, University of Illinois

Abs. 98 Structural Studies of Plant Signal Transduction Proteins A. Grantz, W. Gruissem and S.-H. Kim, Lawrence Berkeley National Laboratory

Abs. 113 Action and Synthesis of Plant Hormones

H. Kende, Michigan State University DOE Plant Research Laboratory

Abs. 114 Photoperiodic Induction and the Floral Stimulus

H. Kende, L. Mclntosh, J.A.D. Zeevaart, Michigan State University DOE Plant Research Laboratory

Abs. 116 Sensory Transduction in Plants

K.L. Poff, Michigan State University DOE Plant Research Laboratory

Abs. 119* Developmental Biology of Nitrogen-fixing Cyanobacteria C.P. Wolk, Michigan State University DOE Plant Research Laboratory

Abs. 121 Environmental Control of Plant Development and Its Relation to Plant Hormones

J.A.D. Zeevaart, Michigan State University DOE Plant Research Laboratory

Abs. 129 CLV Signaling in Meristem Development

S. Clark, University of Michigan

Abs. 137 Targeting and Processing of the Thiol Protease Aleurain J.C. Rogers, University of Missouri 
160 / Division of Energy Biosciences - Summaries of FY 1996 Activities

Abs. 166 Role of $\mathrm{Ca}^{++} /$Calmodulin in the Regulation of Microtubules in Higher Plants R. Cyr, Pennsylvania State University

Abs. 171 Light Responses and Photoperiodism in Arabidopsis thaliana

A.R. Cashmore, University of Pennsylvania

Abs. 179* A Molecular-Genetic Approach to Studying Source-Sink Interactions in Arabidopsis thaliana

S.I. Gibson, Rice University

Abs. 195* Molecular Mechanism of Light-Regulated Protein Transport Across the Nuclear Envelope

A.G. von Arnim, University of Tennessee

Abs. 210 Biochemical and Molecular Analysis of a Transmembrane Protein Kinase from Arabidopsis thaliana

A.B. Bleecker, University of Wisconsin

\section{PLANT GENETIC REGULATION AND GENETIC MECHANISMS}

The most profound advances in biology over the last decade or so have been made in gaining a greater understanding of genetic structure and expression. This has also included the development of modes of transferring genetic information between organisms as well as extremely detailed characterization of genes. In this category, efforts are included to better comprehend how certain complex genetic components are expressed, what the regulatory elements are, and how exterior signals trigger genetic expression. The overall importance of this information in respect to future biotechnological developments rests with the ability to provide ways of assuring expression of desirable genes in plants that will result in improved quantity and quality of products.

Abs. 10 Regulation of DNA Endoreplication in Maize Endosperm

B.A. Larkins, University of Arizona

Abs. 14 Role of HSP100 Proteins in Plant Stress Tolerance

E. Vierling, University of Arizona and S. Lindquist, University of Chicago

Abs. 16 Differential Regulation of Plastid mRNA Stability

D.B. Stern, Boyce Thompson institute for Plant Research, Inc.

Abs. 17 Molecular Plant Genetics

B. Burr and F.A. Burr, Brookhaven National Laboratory 
Abs. 24 Cellular Integration of MVA Synthesis and Protein Prenylation

W. Gruissem, University of California - Berkeley

Abs. 45* Production of Lipophilic Materials from Plants

C.R. Somerville, Carnegie Institution of Washington

Abs. 48 Role of HSP100 Proteins in Plant Stress Tolerance

$\mathrm{S}$. Lindquist, University of Chicago

Abs. 53 The Suppression of Mutations Generated by Mu Transposons in Maize R.A. Martienssen and V. Sundaresan, Cold Spring Harbor Laboratory

Abs. 76 Why Do Plants Have Two Pathways of Polyamine Synthesis?

R.L. Malmberg, University of Georgia

Abs. 77 The Determinants of RNA Turnover in Higher Plants

R.B. Meagher, University of Georgia

Abs. 79 Post-transcriptional Regulation and Evolution of the $R / B$ Gene Family in Maize and Rice

S.R. Wessler, University of Georgia

Abs. 81 Development of Innovative Techniques That May be Used as Models to Improve Plant Performance

W.W. Hanna and G.W. Burton, University of Georgia

Abs. 89 Regulation of Carotenoid Biosynthesis: The immutants Variegation Mutant of Arabidopsis

S. Rodermel and D. Voytas, lowa State University

Abs. 107 Isolation of Genes Involved in a Novel Auxin Biosynthetic Pathway

J. Normanly, University of Massachusetts

Abs. 109 Molecular Mechanisms That Regulate the Expression of Genes in Plants

P. Green, Michigan State University DOE Plant Research Laboratory

Abs. 111 Chloroplast Biogenesis

K. Keegstra, Michigan State University DOE Plant Research Laboratory

Abs. 115 Interaction of Nuclear and Organelle Genomes

L. McIntosh, Michigan State University DOE Plant Research Laboratory

Abs. 134 Dosage Analysis of Gene Expression in Maize

J. Birchler, University of Missouri 
162 / Division of Energy Biosciences - Summaries of FY 1996 Activities

Abs. 135 Position Effect as a Determinant of Variegated Pigmentation in Maize K.C. Cone, University of Missouri

Abs. 136 Molecular Analyses of Nuclear-Cytoplasmic Interactions in the Genus Zea K.J. Newton, University of Missouri

Abs. 147 Map Based Cloning of the Enhancer of Gene Silencing 1 Locus Which Enhances the Silencing of a Foreign Gene in Arabidopsis S.R. Grant, University of North Carolina

Abs. 162 Genetic Analysis of Chloroplast Translation

A. Barkan, University of Oregon

Abs. 170 Structural Basis of Signal and Energy Transduction in Plants A.R. Cashmore, University of Pennsylvania

Abs. 173 Molecular and Genetic Analysis of Hormone-Regulated Differential Cell Elongation in Arabidopsis

J.R. Ecker, University of Pennsylvania

Abs. 185 Corn Storage Protein: A Molecular Genetic Model J. Messing, Rutgers University

Abs. 186 Signal Transduction Pathways that Regulate CAB Gene Expression J. Chory, Salk Institute for Biological Studies

Abs. 187. Genetic Engineering with a Gene Encoding a Soybean Storage Protein R.N. Beachy, The Scripps Research Institute

Abs. 189 Nuclear Genes Regulating Translation of Organelle mRNAs

S. Mayfield, The Scripps Research Institute

Abs. 199 Arabidopsis Genome Sequencing Using Random Shotgun Sequencing of BAC Clones

J.C. Venter, The Institute for Genomic Research

Abs. 207* Enhancement of Photoassimilate Utilization by Manipulation of ADP-glucose pyrophosphorylase

T.W. Okita, Washington State University

Abs. 213 Organization of the $R$ Chromosome in Maize

J. Kermicle, University of Wisconsin 
Abs. 217 Analysis of Structural Domains Required for Phytochrome Function by in vitro Mutagenesis

R.D. Vierstra, University of Wisconsin

Abs. 221 Molecular Genetics of the R-r Complex of Maize

S. Dellaporta, Yale University

\section{PLANT CELL WALL DEVELOPMENT}

The most dominant biomass products are plant cell walls which consist of polysaccharides, lignins, proteins and other compounds. The category includes research on the synthesis, structure, function and other aspects of cell wall components. In approaching the multiple questions encompassed by this topic, a broad diversity of techniques are employed including fast atom bombardment mass spectroscopy, Raman spectroscopy, Nuclear Magnetic Resonance spectroscopy and numerous molecular biological procedures, among others. The objective is to insure our knowledge about this natural resource, which would result in greater productivity and also make it more utilizable.

Abs. 1 Molecular Organization in the Native State of Wood Cell Walls: Studies of Tertiary Structure and its Development Using the Raman Microprobe, Solid State ${ }^{13} \mathrm{C}$ NMR, Fluorescence Spectroscopy and Photoconductivity R.H. Atalla, USDA - Madison, Wisconsin

Abs. 8 Role of Pectolytic Enzymes in the Programmed Release of Cells from the Root Cap of Higher Plants M.C. Hawes, University of Arizona

Abs. 30 Biosynthesis of Cellulose and Callose in Developing Cotton Fibers D.P. Delmer, University of California - Davis

Abs. 54 Analysis of Cell Wall Properties in Polysaccharide Mutants of Arabidopsis W.-D. Reiter, University of Connecticut

Abs. 69 CarbBank - A Structural and Bibliographic Database for Complex Carbohydrates

P. Albersheim and S. Doubet, University of Georgia

Abs. 70 The University of Georgia Complex Carbohydrate Research Center (CCRC) P. Albersheim and A. Darvill, University of Georgia

Abs. 71 The Structures and Functions of Oligosaccharins

P. Albersheim, University of Georgia 
164 / Division of Energy Biosciences - Summaries of FY 1996 Activities

Abs. 72 Structural Studies of Complex Carbohydrates of Plant Cell Walls A. Darvill, University of Georgia

Abs. 73 Mechanisms of Lignin Biosynthesis During Xylogenesis in Zinnia elegans K-E.L. Eriksson and J.F.D. Dean, University of Georgia

Abs. 112 Cell Wall Metabolism

K. Keegstra, H. Kende, N.V. Raikel, and J.D. Walton, Michigan State University DOE Plant Research Laboratory

Abs. 133 Cellulose Synthesis and Morphogenesis

T.I. Baskin, University of Missouri

Abs. 145 Transcription Factors in Xylem Development

R. Sederoff, M. Campbell, R. Whetten and D. O'Malley, North Carolina State University

Abs. 153 The Molecular Characterization of the Lignin-Forming Peroxidase

L.M. Lagrimini, Ohio State University

Abs. 157 The Structure of Pectins from Cotton Suspension Culture Cell Walls A.J. Mort, Oklahoma State University

Abs. 165 Biophysical and Molecular Mechanisms Controlling Plant Cell Growth D.J. Cosgrove, Pennsylvania State University

Abs. 175 Purification and Molecular Cloning of the Synthases of Cereal $(1 \rightarrow 3),(1 \rightarrow 4)-\beta$ d-glucan

N.C: Carpita, Purdue University

Abs. 176 Modification of Lignin Composition in Plants by Manipulation of ferulate-5hydroxylase Expression

C.C.S. Chapple, Purdue University

Abs. 193 Biochemical and Molecular Characterization of Enzymes for Cell Wall Synthesis

P.M. Ray, Stanford University

Abs. 198 Molecular, Genetic, and Biochemical Analysis of Cellulose Synthesis in Arabidopsis thaliana

R.M. Brown, Jr., and K. Sathasivan, University of Texas 
Abs. 205 A Comprehensive Approach to the Elucidation of Lignification at the Plasma Membrane/Cell Wall Interface

N.G. Lewis, Washington State University

Abs. 208 Plant Cell Wall Architecture

Z.-H. Ye, Washington University

\section{LIGNIN-POLYSACCHARIDE BREAKDOWN}

The most abundant biomass consists of complex associations of polymers including lignin, cellulose and other polysaccharides that are not easily broken down to convert these polymers into other usable products. Clearly, organisms have been carrying out such conversions for econs, but the exact details of the mechanisms are lacking. Accordingly, this category dwells on basic studies dealing with the organismal and enzymatic conversion of the major constituents of lignocellulosic biomass into usable feedstocks. Such studies include genetic, biochemical and physiological approaches in learning how organisms do this so that it may be possible to carry forth these processes in a biotechnological manner.

Abs. $31 \quad$ Cellulose Binding Proteins of Clostridium cellulovorans Cellulase R.H. Doi, University of California - Davis

Abs. 61 Studies of the Genetic Regulation of the Thermomonospora fusca Cellulase Complex

D.B. Wilson, Cornell University

Abs. 75 Fermentation of Cellulose and Hemicelluloses by Clostridia and Anaerobic Fungi

L.G. Ljungdahl, University of Georgia

Abs. 106 Cellulose Fermentation by Nitrogen-Fixing Anaerobic Bacteria S. Leschine, University of Massachusetts

Abs. $122 \quad$ Xylan-Degrading Enzymes of Cytophaga xylanolytica J.A. Breznak, Michigan State University

Abs. 127 Physiology and Molecular Biology of Lignin-Modifying Enzymes C.A. Reddy, Michigan State University

Abs. 159 Biochemical Genetics of Lignin Degradation by Phanerochaete chrysosporium

M.H. Gold, Oregon Graduate Institute of Science \& Technology 
166 / Division of Energy Biosciences - Summaries of FY 1996 Activities

Abs. 160 Cellobiose Dehydrogenase and $\beta$-glucosidase from Phanerochaete chrysosporium: Effect on Cellulose Hydrolysis, Cloning, and Characterization

V. Renganathan, Oregon Graduate Institute of Science \& Technology

Abs. 169 Characterization of Lignin and Mn Peroxidases from Phanerochaete chrysosporium

M. Tien, Pennsylvania State University

Abs. 181 Synergism and Interaction Between Clostridium thermocellum Major Cellulosome Components, CelS and Cell

J.H.D. Wu, University of Rochester

Abs. 211 Molecular Genetics of Ligninase Expression

D. Cullen, University of Wisconsin

Abs. 212 Identification of the Primary Mechanism for Fungal Lignin Degradation K.E. Hammel, University of Wisconsin

\section{NITROGEN FIXATION AND PLANT-MICROBIAL SYMBIOSIS}

One of the most crucial aspects of plant nutrition is obtaining adequate nitrogen for the growth and survival. Nature has evolved the technique of nitrogen fixation for using atmospheric nitrogen to supply the needs of microbes and certain plants via symbiotic reactions. The prospect of transferring the mechanism to avoid the use of man-made nitrogen fertilizer has been suggested, but unless the whole process of nitrogen fixation in microbes and in symbiotic relationships is better understood, no such objective can ever be considered seriously. The projects in this category cover various aspects of these processes in both microbes and plants, including studies on nodulation, reactions and other topics.

Abs. 9 Rhizosphere Association of the Nitrogen Fixing Bacterial Species Azotobacter paspali with the Tropical Grass Papalum notatum: Specificity and Significance to Plant Nutrition

C. Kennedy, University of Arizona

Abs. 43 Regulation and Function of Two Cell Wall Protein Genes in Medicago Roots and Root Nodules

J.B. Cooper, University of California - Santa Barbara

Abs. 47 Organization and Regulation of the Genes for Nitrogen Fixation in Rhodobacter capsulatus

R. Haselkorn, University of Chicago 
Abs. 108 Molecular Basis of Symbiotic Plant-Microbe Interactions

F.J. de Bruijn, Michigan State University DOE Plant Research Laboratory

Abs. 119* Developmental Biology of Nitrogen-Fixing Cyanobacteria

C.P. Wolk, Michigan State University DOE Plant Research Laboratory

Abs. $120 \quad$ Frankia Genetics

C.P. Wolk, T. Newman, S.Y. He and F.J. de Bruijn, Michigan State

University DOE Plant Research Laboratory

Abs. 123 The Role of Bacterial Surface Glycoconjugates in the Rhizobium/Legume Symbiosis

R.I. Hollingsworth, Michigan State University

Abs. 161* Catalytic Mechanism of Hydrogenase from Aerobic $\mathrm{N}_{2}$-Fixing Microorganisms

D.J. Arp, Oregon State University

Abs. 192 Nodulation Genes and Factors in the Rhizobium-Legume Symbiosis

S.R. Long, Stanford University

Abs. 194 Plant Recognition of Bradyrhizobium japonicum Nod Factors

G. Stacey, University of Tennessee

Abs. 204* Carbon Metabolism in Symbiotic Nitrogen Fixation

M.L. Kahn, Washington State University

Abs: 223 Role of Phenolics in the Adaptation of Rhizobia to the Rhizosphere and Soil D. Parke and L.N. Ornston, Yale University

\section{MECHANISMS FOR PLANT ADAPTATION}

Plant survival frequently depends on the ability to detect and resist attack from other organisms as well as the ability to grow under sub-optimal environmental conditions. How plants withstand pests and pathogens and contend with drought conditions, heat, salinity and other factors that deter growth is the emphasis of this category. Studies are aimed at discerning the mechanisms by which plants detect, adapt to, and survive such conditions. Once again effects using genetic, biochemical and physiological approaches are revealing the basic mechanisms plants employ to mitigate the organisms and environmental conditions that threaten their growth. 
168 / Division of Energy Biosciences - Summaries of FY 1996 Activities

Abs. 13 Phytoalexin Detoxification Genes and Gene Products: Implication for the Evolution of Host Specific Traits for Pathogenicity

H. VanEtten, University of Arizona

Abs. 25 Determinants of Environmental Stress Tolerance by Bacteria on Leaves S.E. Lindow, University of California - Berkeley

Abs. 28 Molecular Cloning and Characterization of the Arabidopsis thaliana RPS2 Disease Resistance Locus

B.J. Staskawicz, University of California - Berkeley

Abs. $34^{\star} \quad$ Cellular and Molecular Characterization of Vascular Plasmodesmata W.J. Lucas, University of California - Davis

Abs. 42* Sensory Transduction of the $\mathrm{CO}_{2}$ Response of Guard Cells

E. Zeiger, University of California - Los Angeles

Abs. 46 Powdery Mildew Disease Resistance

S.C. Somerville, Carnegie Institution of Washington

Abs. 49 Signal Transduction in Plant Development: Chemical and Biochemical Approaches to Receptor Identification

D.G. Lynn, University of Chicago

Abs. 110 Molecular Biology of Plant-Bacterial Interactions

S.Y. He, Michigan State University DOE Plant Research Laboratory

Abs. 118 Biochemical and Molecular Aspects of Plant Pathogenesis

J.D. Walton, Michigan State University DOE Plant Research Laboratory

Abs. 141 Tomato Bushy Stunt Virus and DI RNAs as a Model for Studying Mechanisms of RNA Virus Replication, Pathogenicity and Recombination T.J. Morris, University of Nebraska and A.O. Jackson, University of California, Berkeley

Abs. 146 Novel Control of Signal Delivery from the Pseudomonas syringae avrRPM1 Gene to Arabidopsis thaliana

J.L. Dangl, University of North Carolina

Abs. 182 Characterization of the Systemic Acquired Resistance Immediate-Early Response to Salicylic Acid

N.-H. Chua, Rockefeller University 
Abs. 184 Respiration and Active Oxygen Species Production in Stressed Plants I. Raskin, Rutgers University

\section{FERMENTATIVE MICROBIAL METABOLISM}

Organisms that live in the absence of atmospheric oxygen oftentimes have unique biochemical pathways including different energy deriving reactions. The projects included in this category focus on attempting to understand the nature of some of these pathways including the unusual pattern of degradation of aromatic compounds as well as polysaccharide breakdown, production of organic solvents and others. Such basic knowledge could afford new ways of converting biomass resources into useful products and also give insights into ways of degrading certain pollutants for passible introduction into bioremediation. This is one of the several areas within the EB program which has been understudied.

Abs. 66 Ethanologenic Enzymes of Zymomonas mobilis L.O. Ingram, University of Florida

Abs. 90 Molecular Biology of Anaerobic Aromatic Biodegradation C.S. Harwood, University of lowa

Abs. 131 The Mechanism of Switching from an Acidogenic to a Butanol-Acetone Fermentation by Clostridium acetobutylicum

P. Rogers, University of Minnesota

Abs. 158 Energetic's and Kinetics of Syntrophic Aromatic Degradation M.J. Mclnerney, University of Oklahoma

Abs. 161* Catalytic Mechanism of Hydrogenase from Aerobic $\mathrm{N}_{2}$-Fixing Microorganisms

D.J. Arp, Oregon State University

Abs. 178 Genetic and Biochemical Analysis of Solvent Formation in Clostridium acetobutylicum

G.N. Bennett and F.B. Rudolph, Rice University

Abs. 191 Regulation of Alcohol Fermentation by Escherichia coli

D.P. Clark, Southern Illinois University

Abs. 200 Enzymology of Acetone-Butanol-Isopropanol Formation

J.-S. Chen, Virginia Polytechnic Institute and State University 


\section{ONE AND TWO CARBON MICROBIAL METABOLISM}

Microorganisms, particularly anaerobic ones, are greatly attuned to the conversion of carbon compounds such as carbon monoxide, acetic acid, methanol and others. For example, the production of methane in the very large number of varied sites e.g., swamps, rumens, rice paddies largely involves using one or two carbon precursor molecules on the part of the methanogens involved. It is another area of much needed attention to be able to understand the types of conversions that the large numbers of organisms are capable of. With such information in hand and with the capabilities of genetic manipulation now possible entirely new bioconversion resources may be feasible. Thus the studies covered include methanogenesis, methylotrophy and other systems.

Abs. $15^{*}$ Osmoregulation in Methanogens

M.F. Roberts, Boston College

Abs. 40 Molecular Biology and Genetics of the Acetate-Utilizing Methanogenic Bacteria

R.P. Gunsalus, University of California - Los Angeles

Abs. 62 Conversion of Acetic Acid to Methane by Thermophiles

S.H. Zinder, Cornell University

Abs. 80 Biochemistry and Genetics of Autotrophy in Methanococcus

W.B. Whitman, University of Georgia

Abs. 86 Exploratory Studies on the Bacterial Formation of Methane

R.S. Wolfe, University of llinois

Abs. 88 Characterization of the Membrane-Associated Methane Oxidation System in Methylococcus capsulatus Bath

A.A. DiSpirito, lowa State University

Abs. 94 Acetyl-CoA Cleavage and Synthesis in Methanogens: Mechanistic, Enzymological, and Metabolic Studies

E. DeMoll, University of Kentucky and D.A. Grahame, Uniformed Services University of the Health Sciences

Abs. 102 Carbon Metabolism in Methylotrophic Bacteria

C.J. Unkefer, Los Alamos National Laboratory

Abs. 104 Regulation of Catabolic Gene Transcription in the Methanogenic Archaea K.R. Sowers, University of Maryland 
Abs. 128 Molecular Physiology of Succinic Acid-Based Fermentations in Anaerobes: Control of Chemical Yield by $\mathrm{CO}_{2}$ Fixation and Electron Donors J.G. Zeikus, Michigan State University

Abs. 130 Genetics of Bacteria that Utilize One-Carbon Compounds

R.S. Hanson, University of Minnesota

Abs. 142 Enzymology of Aceticlastic Methanogenesis

S.W. Ragsdale, University of Nebraska

Abs. 152 Transmethylation Reactions During Methanogenesis from Acetate or Methylamines in Methanosarcina barkeri

J.A. Krzycki, Ohio State University

Abs. 154 Structure and Regulation of Methane Genes

J.N. Reeve, Ohio State University

Abs. 167 Enzymological Studies of One-Carbon Reactions in the Pathway of Acetate Utilization by Methanogenic Bacteria

J.G. Ferry, Pennsylvania State University

Abs. 209 Genetics in Methylotrophic Bacteria

M.E. Lidstrom, University of Washington

Abs. 214 The Biochemistry, Bioenergetics and Physiology of the CO-dependent Growth of Rhodospirillum rubrum

P.W. Ludden, University of Wisconsin

\section{EXTREMOPHILIC MICROBES}

How microorganisms cope with exceedingly high temperatures, alkalinity, salinity and other factors is an intriguing serious of questions. Ordinary life succumbs to such conditions. What mechanisms are there that afford the microbes the ability not only to survive, but also to grow under harsh conditions? This is the theme of this category in which a variety of approaches are being employed. The potential benefit of such information generated could be in designing new enzymes that are capable of activity under the same conditions as part of new biotechnological developments.

Abs. 26 Phylogenetic Analysis of Hyperthermophilic Natural Populations Using Ribosomal RNA Sequences

N.R. Pace, University of California - Berkeley 
172 / Division of Energy Biosciences - Summaries of FY 1996 Activities

Abs. 37 Membrane Bioenergetics of Salt Tolerant Microorganisms J.K. Lanyi, University of California - Irvine

Abs. 55 Sugar Transport and Metabolism in Thermotoga A.H. Romano and K.M. Noll, University of Connecticut

Abs. 68 The Metabolism of Hydrogen by Extremely Thermophilic Bacteria M.W.W. Adams, University of Georgia

Abs. 103 Structure and Regulation of L-glutamate Dehydrogenase in Hyperthermophilic Archaea

F.T. Robb and J. DiRuggiero, University of Maryland

Abs. 144 Bioenergetic and Physiological Studies of Hyperthermophilic Archaea R.M. Kelly, North Carolina State University

Abs. 149 In vivo Analysis of Archael Transcription Signals and the Regulation of Heat Shock Promoters C. Daniels, Ohio State University

Abs. 163 The Characterization of Psychrophilic Microorganisms and Their Potentially Useful Cold-Active Glycosidases

J.E. Brenchley, Pennsylvania State University

Abs. 180 Structural Studies of Archaeal Thermophilic Adenylate Kinase J.Konisky, Rice University

\section{MICROBIAL RESPIRATION, NUTRITION AND METAL METABOLISM}

In this category some of the unusual characteristics of microbes are under investigation. The ability to switch from aerobic to anaerobic metabolism is one question being pursued with the consideration of bioenergetics as part of the studies. Others deal with how certain organisms handle heavy metal ions as part of their metabolism. This type of information could bear on a variety of microbial biotechnology developments.

Abs. 58 Regulation of Denitrification in Rhodobacter sphaeroides J.P. Shapleigh, Cornell University

Abs. 60 Genetic Control of Nitrate Assimilation in Klebsiella pneumoniae V.J. Stewart, Cornell University 
Abs. 83 Heavy Metal-lux Sensor Fusions and Gene Regulation

S. Silver, University of Illinois - Chicago

Abs. 84 Studies on the $\mathrm{bo}_{3}$-type Ubiquinol Oxidase from Escherichia coli

R.B. Gennis, University of Illinois

Abs. 91 Bacterial Nickel Metabolism and Storage

R.J. Maier, Johns Hopkins University

Abs. 132 Isolation and Characterization of Ammonia Monoxygenase of Nitrosomonas

A.B. Hooper, University of Minnesota

Abs. 138 Genetics of the Sulfate-Reducing Bacteria

J.D. Wall and B.J. Rapp-Giles, University of Missouri

Abs. 139 The Respiratory Chain of Alkaliphilic Bacteria

T.A. Krulwich, Mount Sinai School of Medicine

Abs. 150 Mechanisms of Microbial Adaptation

C. Daniels and W. Strohl, Ohio State University

Abs. 151* Biosynthesis of Hydrocarbons

P.E. Kolattukudy, Ohio State University

Abs. 196 Role of Glycolytic Intermediations in Global Regulation and Signal

Transduction

J.C. Liao, Texas A\&M University

Abs. 219 Molecular Characterization of Bacterial Respiration on Minerals

R. Blake II, Xavier University of Louisiana

Abs. 220 Biochemistry of Dissimilatory Sulfur Oxidation

R. Blake II, Xavier University of Louisiana

\section{MATERIALS BIOSYNTHESIS}

The prospects of developing entirely new materials using either organisms or enzymes in the synthesis is a prospect of great interest. In collaboration with the Materials Sciences Division of the Office of Basic Energy Sciences efforts are being put into studies that could result in the synthesis of new enzymes, polymers and other substances using biological precursors and ideas as the basis. 
174 / Division of Energy Biosciences - Summaries of FY 1996 Activities

Abs. 97 Enzymatic Synthesis and Biomolecular Materials M.D. Alper, C. Bertozzi, D. Charych, J.F. Kirsch, D.E. Koshland, P.G. Schultz, R. Stevens, F. Tropper and C.-H. Wong, Lawrence Berkeley National Laboratory

Abs. $218 \quad$ Novel Biomaterials: Genetically Engineered Pores

H. Bayley, Worcester Foundation for Biomedical Research 


\section{INVESTIGATOR INDEX}

Adams, M.W.W -- University of Georgia . . . . . . . . . . . . . . . . . . 68

Albersheim, P. - University of Georgia . . . . . . . . . . . . . . . . . . . . 69,70,71

Alper, M.D. -- Lawrence Berkeley National Laboratory . . . . . . . . . . . . . . . . . 97

Arp, D.J. -- Oregon State University . . . . . . . . . . . . . . . . . . 161

Atalla, R.H. -- U.S. Department of Agriculture . . . . . . . . . . . . . . . 1

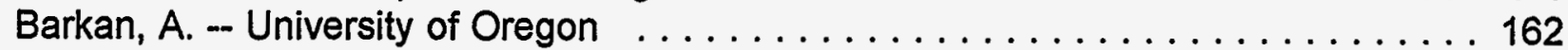

Baskin, T.I. -- University of Missouri . . . . . . . . . . . . . . . . . . . . 133

Bayley, H. -- Worcester Foundation for Biomedical Research . . . . . . . . . . . . . 218

Beachy, R.N. -- The Scripps Research Institute . . . . . . . . . . . . . . . . 187

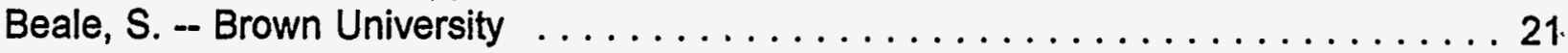

Bennett, G.N. -- Rice University . . . . . . . . . . . . . . . . . . . . . 178

Bertozzi, C. -- Lawrence Berkeley National Laboratory . . . . . . . . . . . . . . . . . . . 97.

Birchler, J. -- University of Missouri . . . . . . . . . . . . . . . . . . . . . . . . . 134

Blake, R., II -- Xavier University of Louisiana . . . . . . . . . . . . . . . . . 219,220

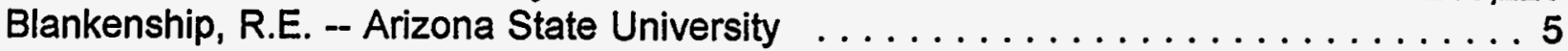

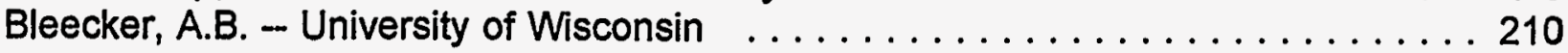

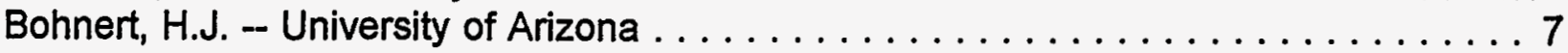

Boyer, J.S. -- University of Delaware $\ldots \ldots \ldots \ldots \ldots \ldots$

Boynton, J.E. -- Duke University . . . . . . . . . . . . . . . . . . . . . 64

Brenchley, J.E. - Pennsylvania State University . . . . . . . . . . . . 163

Breznak, J.A. - Michigan State University . . . . . . . . . . . . . . . . . . . . . . . . 122

Brown, R.M., Jr. -. University of Texas . . . . . . . . . . . . . . . . . . . . 198

Browse, J. . Washington State University . . . . . . . . . . . . . . . . . . . . . . 202

Bryant, D.A. -- Pennsylvania State University . . . . . . . . . . . . . . . . . . . 164

Burr, B. - Brookhaven National Laboratory . . . . . . . . . . . . . . . . . . 17

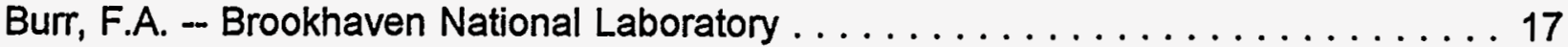

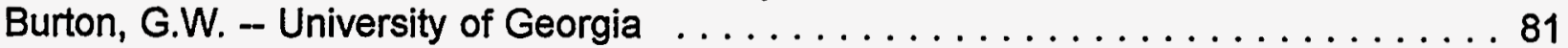

Bush, D.R. .- University of Illinois . . . . . . . . . . . . . . . . . 87

Campbell, M. -- North Carolina State University . . . . . . . . . . . . . . . . . 145

Carpita, N.C. -- Purdue University . . . . . . . . . . . . . . . . . . . 175

Cashmore, A.R. -- University of Pennsylvania . . . . . . . . . . . . . . . . 170,171

Chapple, C.C.S. -. Purdue University . . . . . . . . . . . . . . . . . . . 176

Charych, D. -- Lawrence Berkeley National Laboratory ... . . . . . . . . . . . . . 97

Cheeseman, J.M. -- University of Illinois . . . . . . . . . . . . . . . . . . . 87

Chen, J.-S. -- Virginia Polytechnic Institute and State University . . . . . . . . . . . . 200

Chin, C. -. Rutgers University . . . . . . . . . . . . . . . . . . . . . . 183

Chory, J. -- Salk Institute for Biological Studies . . . . . . . . . . . . . . . . . 186

Chrispeels, M.J. -- University of California - San Diego . . . . . . . . . . . . . 38

Chua, N.-H. .- Rockefeller University . . . . . . . . . . . . . . . . . . . . . . 182 
Clark, D.P. -- Southern Illinois University $\ldots \ldots \ldots \ldots \ldots \ldots \ldots \ldots \ldots \ldots \ldots \ldots$

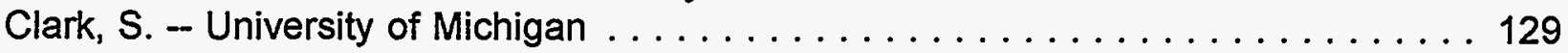

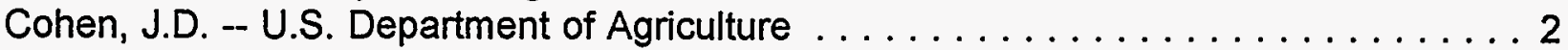

Cone, K.C. - University of Missouri . . . . . . . . . . . . . . . . . . . . . . 135

Conway, T. -- Ohio State University . . . . . . . . . . . . . . . . . . . 148

Cooper, J.B. - University of California - Santa Barbara . . . . . . . . . . . 43

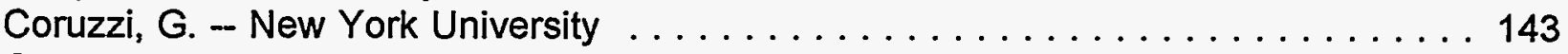

Cosgrove, D.J. -- Pennsylvania State University . . . . . . . . . . . . . . 165

Crofts, A.R. -- University of Illinois $\ldots \ldots \ldots \ldots \ldots \ldots \ldots \ldots \ldots \ldots \ldots \ldots \ldots$

Croteau, R. -- Washington State University . . . . . . . . . . . . . . . . . . . 203

Crouch, R.K. - Medical University of South Carolina . . . . . . . . . . . . . 190

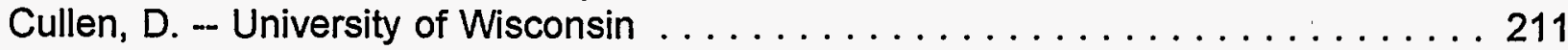

Cyr, R. -- Pennsylvania State University . . . . . . . . . . . . . . 166

Daldal, F. -- University of Pennsylvania $\ldots \ldots \ldots \ldots \ldots \ldots \ldots \ldots \ldots \ldots \ldots$

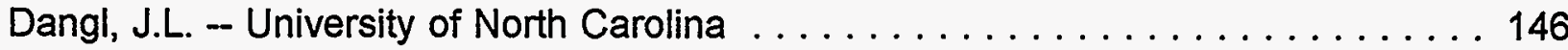

Daniels, C. -- Ohio State University . . . . . . . . . . . . . . . . . . . . 149,150

Darvill, A. - University of Georgia $\ldots \ldots \ldots \ldots \ldots \ldots \ldots \ldots \ldots \ldots \ldots, \ldots \ldots \ldots, 72$

Dean, J.F.D. -- University of Georgia . . . . . . . . . . . . . . . . . . . .73

de Bruijn, F.J. -- Michigan State University DOE Plant Research Laboratory . . 108,120

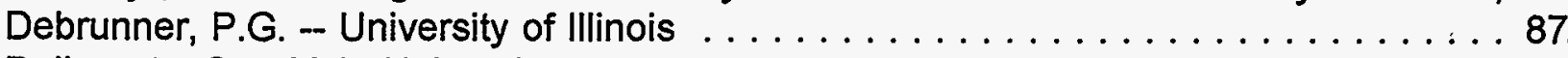

Dellaporta, S. -- Yale University . . . . . . . . . . . . . . . . . . . 221

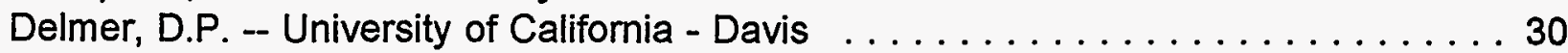

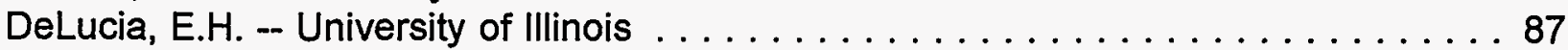

DeMoll, E. -- University of Kentucky . . . . . . . . . . . . . . . . . . . 94

DiRuggiero, J. -- University of Maryland . . . . . . . . . . . . . . . . . . . 103

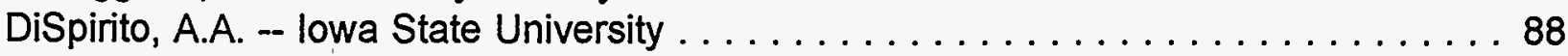

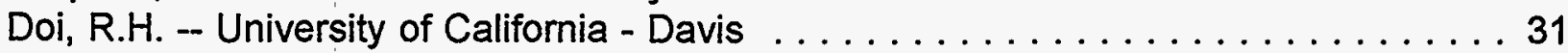

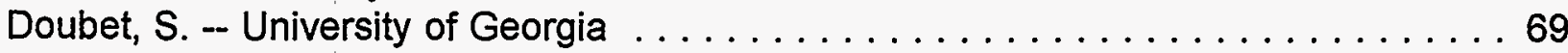

Ecker, J.R. - University of Pennsylvania . . . . . . . . . . . . . . . . 173

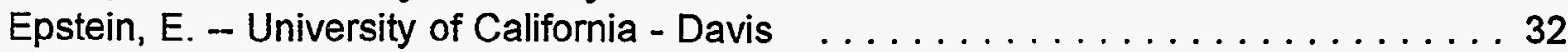

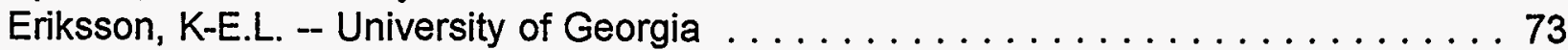

Falkowski, P.G. -- Brookhaven National Laboratory . . . . . . . . . . . . . 18

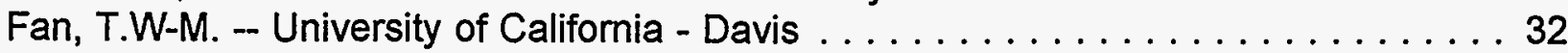

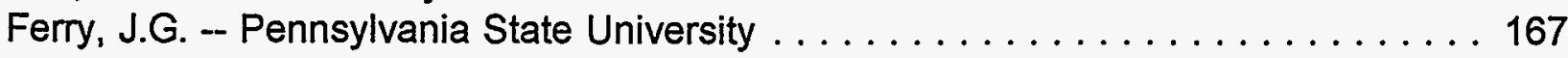

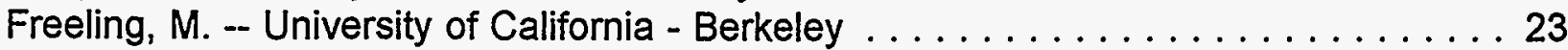

Galau, G.A. -- University of Georgia $\ldots \ldots \ldots \ldots \ldots \ldots \ldots \ldots \ldots \ldots \ldots$

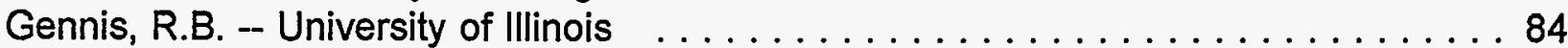

Gibson, S.I. -- Rice University . . . . . . . . . . . . . . . . . . . . . . . 179

Gillham, N.W. -- Duke University . . . . . . . . . . . . . . . . 64

Gold, M.H. - Oregon Graduate Institute of Science and Technology . . . . . . . . 159

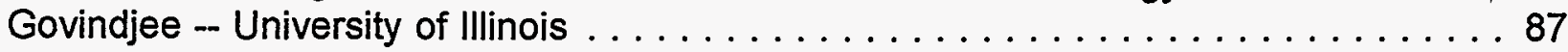

Grahame, D.A. - Uniformed Services University of the Health Sciences . . . . . . . 94

Grant, S.R. -- University of North Carolina . . . . . . . . . . . . . . . . . . . . . 147

Grantz, A. -- Lawrence Berkeley National Laboratory . . . . . . . . . . . . . . 98 
Green, P. -- Michigan State University DOE Plant Research Laboratory . . . . . . 109 Gruissem, W. -- University of California - Berkeley . . . . . . . . . . . . . . 24,98

Guiltinan, M. -. Pennsylvania State University . . . . . . . . . . . . . . . . 168

Gunsalus, R.P. -- University of California - Los Angeles . . . . . . . . . . . . . . 40

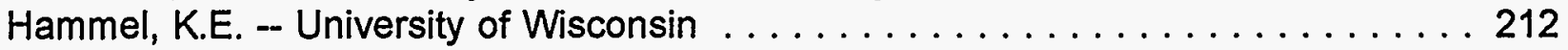

Hanna, W.W. -- University of Georgia . . . . . . . . . . . . . . . . . 81

Hanson, R.S. -- University of Minnesota . . . . . . . . . . . . . . . . . . 130

Hanson, M.R. -- Cornell University . . . . . . . . . . . . . . . . . . 56

Harada, J.J. - University of California - Davis . . . . . . . . . . . . . . . 33

Harper, J. - The Scripps Research Institute . . . . . . . . . . . . . . 188

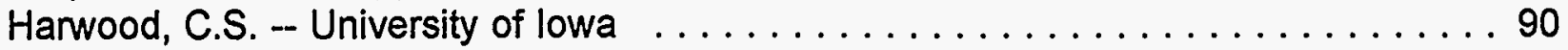

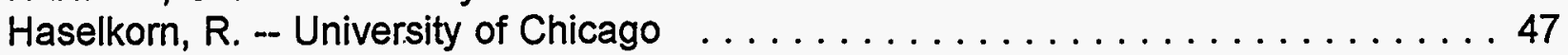

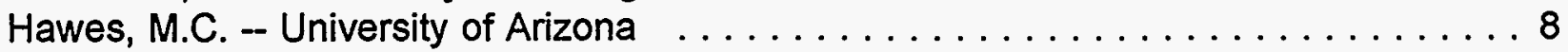

He, S.Y. -- Michigan State University DOE Plant Research Laboratory . . . . . . 110,120

Hearst, J.E. -- Lawrence Berkeley National Laboratory . . . . . . . . . . . . . . . 99

Higashi, R.M. -- University of California - Davis . . . . . . . . . . . . . . 32

Hind, G. -- Brookhaven National Laboratory . . . . . . . . . . . . . . . . . . 19

Hollingsworth, R.I. -- Michigan State University . . . . . . . . . . . . . . . 123

Hooper, A.B. -- University of Minnesota . . . . . . . . . . . . . . . . . . . 132

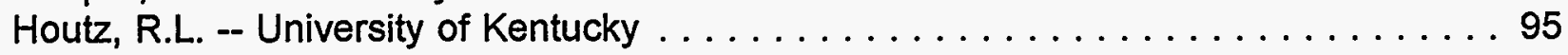

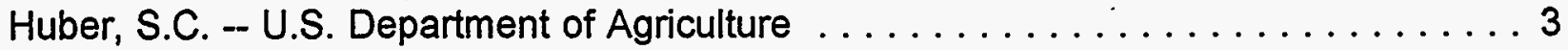

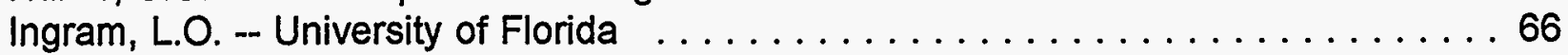

Jackson, A.O. -- University of California . . . . . . . . . . . . . . . . . . 141

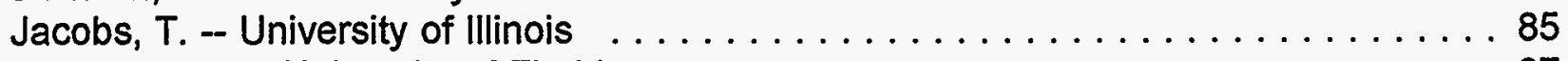

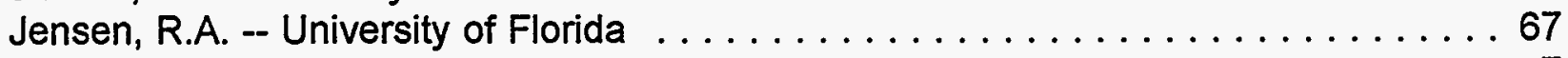

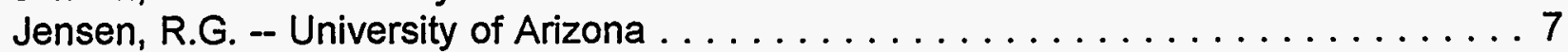

Kahn, M.L. -- Washington State University . . . . . . . . . . . . . . . . . . . . . . 204

Keegstra, K. -- Michigan State University DOE Plant Research Laboratory . . . 111,112

Kelly, R.M. -- North Carolina State University . . . . . . . . . . . . . . . . . . . . . 144

Kende, H. -- Michigan State University DOE Plant Research Laboratory . . 112,113,114

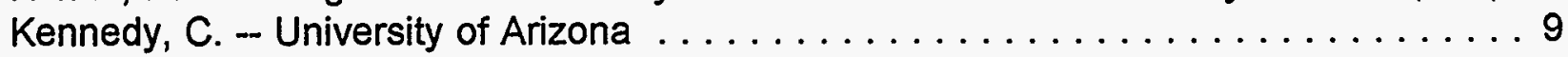

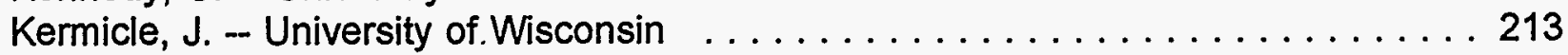

Kim, S.-H. -- Lawrence Berkeley National Laboratory . . . . . . . . . . . . . . . . 98

Kirsch, J.F. -- Lawrence Berkeley National Laboratory . . . . . . . . . . . . . . . . 97

Klein, M.P. - Lawrence Berkeley National Laboratory . . . . . . . . . . . . . . . . 100

Knaff, D.B. - Texas Tech University . . . . . . . . . . . . . . . . . . . . . . . . . 197

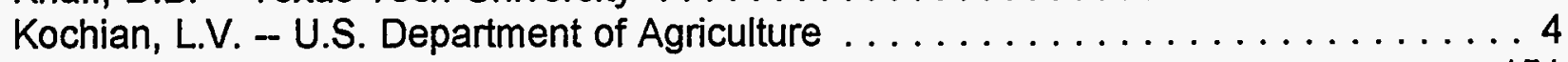

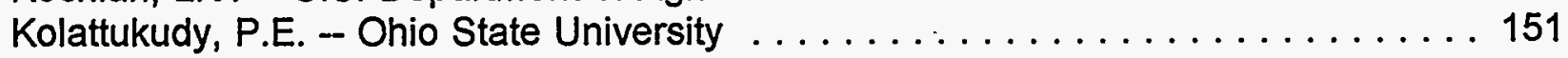

Konisky, J. -- Rice University . . . . . . . . . . . . . . . . . . . . . . . 180

Koshland, D.E. -- Lawrence Berkeley National Laboratory . . . . . . . . . . . . . . . 97

Krulwich, T.A. - Mount Sinai School of Medicine . . . . . . . . . . . . . . . 139

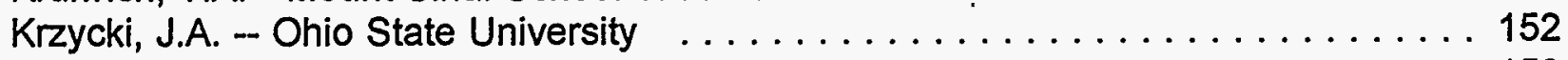

Lagrimini, L.M. -- Ohio State University . . . . . . . . . . . . . . . . . . 153

Lanyi, J.K. - University of California - Irvine . . . . . . . . . . . . . 37 


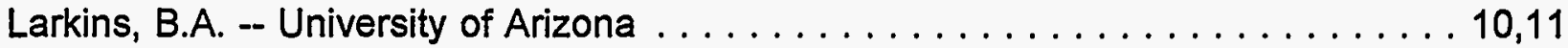

LaRoche, J. -- Brookhaven National Laboratory . . . . . . . . . . . . . . . . 18

Leschine, S. -- University of Massachusetts . . . . . . . . . . . . . . . . . 106

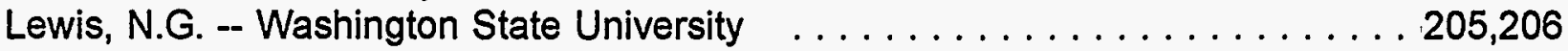

Liao, J.C. -- Texas A\&M University . . . . . . . . . . . . . . . . . . . . . 196

Lidstrom, M.E. -- University of Washington $\ldots \ldots \ldots \ldots \ldots \ldots \ldots 20 \ldots \ldots$

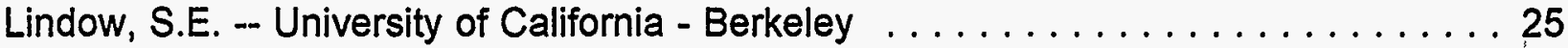

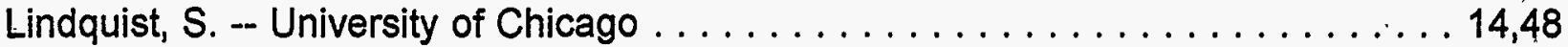

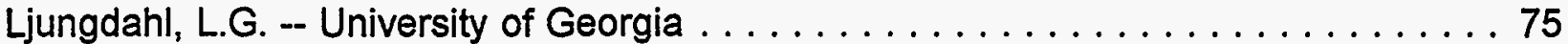

Long, S.R. - Stanford University . . . . . . . . . . . . . . . . . . . . . . . 192

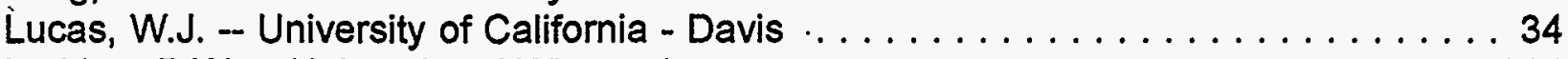

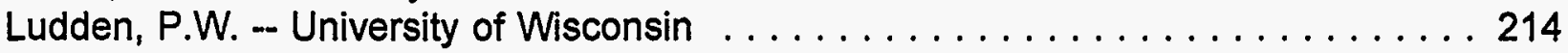

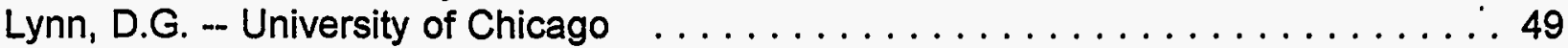

MacMillan, J. - University of California - Los Angeles . . . . . . . . . . . . . 41

Maier, R.J. -- Johnis Hopkins University $\ldots \ldots \ldots \ldots \ldots \ldots \ldots \ldots \ldots \ldots . \ldots \ldots$

Malmberg, R.L. -- University of Georgia $\ldots \ldots \ldots \ldots \ldots \ldots \ldots \ldots \ldots \ldots \ldots$

Martienssen, R.A. -- Cold Spring Harbor Laboratory $\ldots \ldots \ldots \ldots \ldots \ldots \ldots \ldots$

Mayfield, S. -- The Scripps Research Institute . . . . . . . . . . . . . . 189

McCarty, R.E. -- Johns Hopkins University $\ldots \ldots \ldots \ldots \ldots \ldots \ldots \ldots \ldots \ldots \ldots \ldots \ldots$

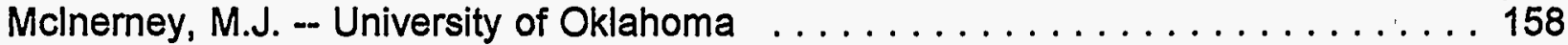

McIntosh, L. -- Michigan State University DOE Plant Research Laboratory . . . 114,115

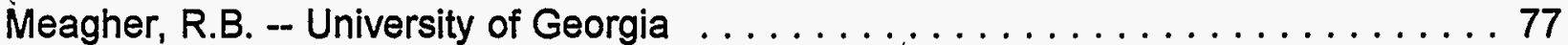

Messing, J. -. Rutgers University ... . . . . . . . . . . . . . . . . . . . 185

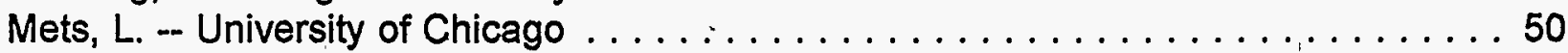

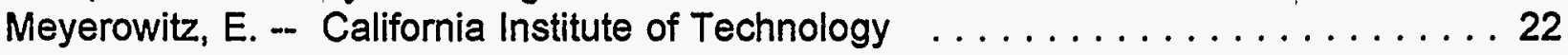

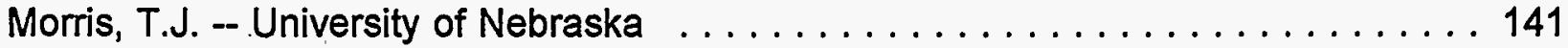

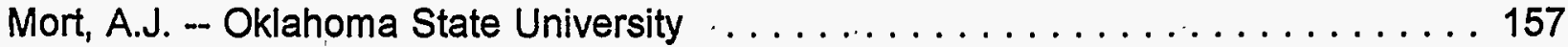

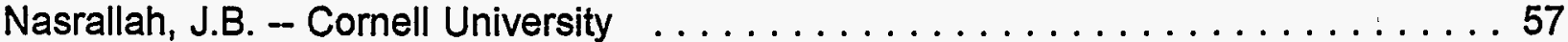

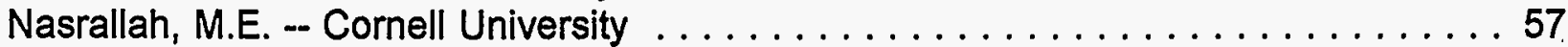

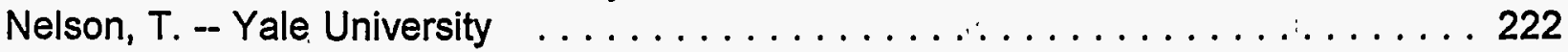

Newman, T. -- Michigan State University DOE Plant Research Laboratory . . . . . . 120

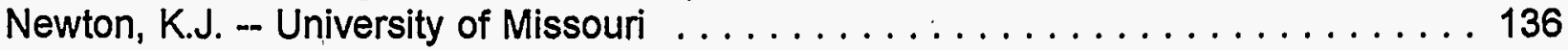

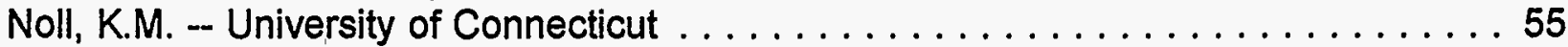

Normanly, J. -- University of Massachusetts . . . . . . . . . . . . . . . 107

Ohlrogge, J. -- Michigan State University . . . . . . . . . . . . . . . . 124,125

Okita, T.W. -- Washington State University . . . . . . . . . . . . . . . 207

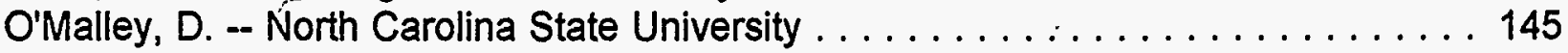

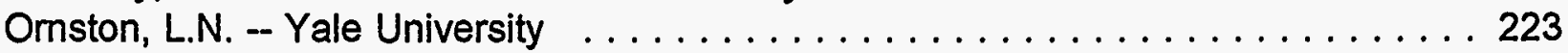

Ort, D.R. -- University of Illinois $\ldots \ldots \ldots \ldots \ldots \ldots \ldots \ldots \ldots \ldots \ldots \ldots \ldots \ldots$

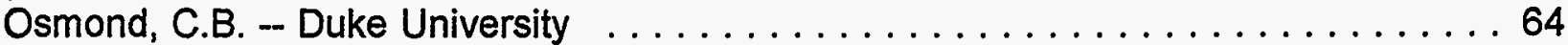

Outlaw, W.H., Jr. -- Florida State University . . . . . . . . . . . . . . 65

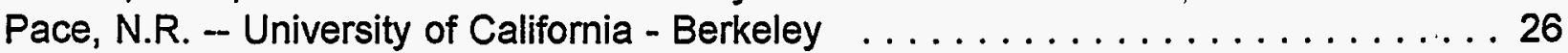

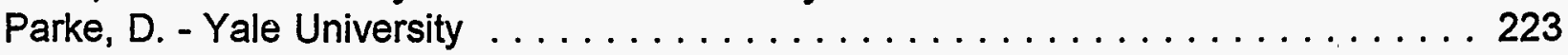

Phinney, B.O. -. University of California - Los Angeles ............. 41 
Poff, K.L. - Michigan State University DOE Plant Research Laboratory . . . . . . . 116

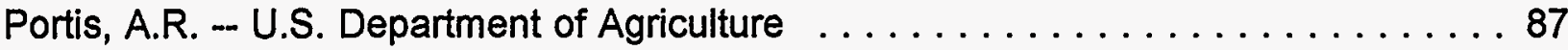

Preiss, J. -- Michigan State University . . . . . . . . . . . . . . . . . . . . . . 126

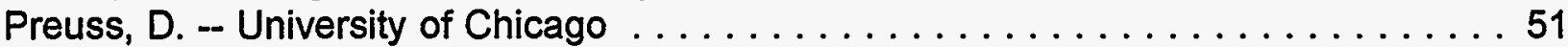

Quail, P.H. -- University of California - Berkeley . . . . . . . . . . . . . . 27

Ragsdale, S.W. -- University of Nebraska . . . . . . . . . . . . . . . . . . . . . 142

Raikhel, N.V. - Michigan State University DOE Plant Research Laboratory . . . 112,117

Rapp-Giles, B.J. -- University of Missouri . . . . . . . . . . . . . . . . . . . 138

Raskin, I. -- Rutgers University . . . . . . . . . . . . . . . . . . . . . 184

Ray, P.M. - Stanford University . . . . . . . . . . . . . . . . . . . . . . . . . 193

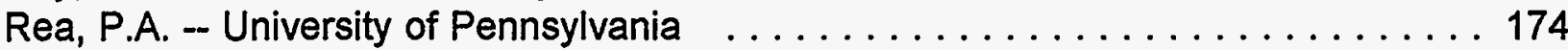

Reddy, C.A. -- Michigan State University $\ldots \ldots \ldots \ldots \ldots \ldots \ldots \ldots$

Reeve, J.N. - Ohio State University . . . . . . . . . . . . . . . . . . . . 154

Reiter, W.-D. -- University of Connecticut . . . . . . . . . . . . . . . . . . . . . . 54

Renganathan, V. -- Oregon Graduate Institute of Science and Technology . . . . . . 160

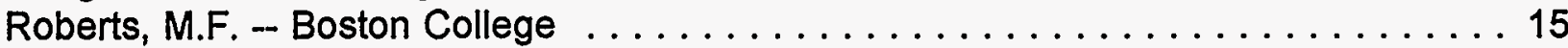

Robb, F.T. - University of Maryland . . . . . . . . . . . . . . . . . . . 103

Rodermel, S. -- lowa State University . . . . . . . . . . . . . . . . . . . . . 89

Romano, A.H. - University of Connecticut . . . . . . . . . . . . . . . . . 55

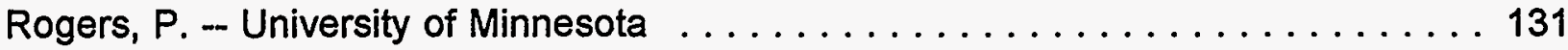

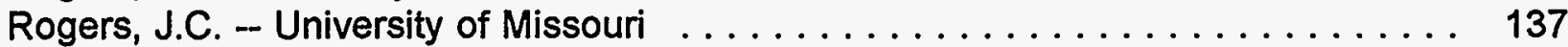

Rudolph, F.B. -- Rice University . . . . . . . . . . . . . . . . . . 178

Sathasivan, K. - University of Texas . . . . . . . . . . . . . . . . . . . . 198

Sauer, K. -- Lawrence Berkeley National Laboratory . . . . . . . . . . . . . . . . . 101

Sayre, R.T. - Ohio State University . . . . . . . . . . . . . . . . . . . 155

Schmidt, G.W. -- University of Georgia $\ldots \ldots \ldots \ldots \ldots \ldots \ldots \ldots$

Schroeder, J.l. -- University of California - San Diego . . . . . . . . . . . . . . . 39

Schultz, P.G. - Lawrence Berkeley National Laboratory . . . . . . . . . . . . . . . . 97.

Schumaker, K.S. -- University of Arizona . . . . . . . . . . . . . . . . . . . . . . . 12

Sederoff, R. -- North Carolina State University . . . . . . . . . . . . . . . . . . . . 145

Seibert, M. -- National Renewable Energy Laboratory . . . . . . . . . . . . . . . . 140

Shanklin, J. -- Brookhaven National Laboratory . . . . . . . . . . . . . . . 20

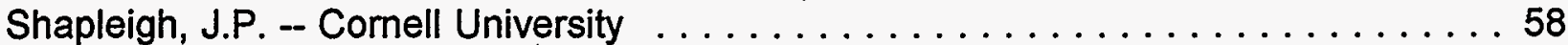

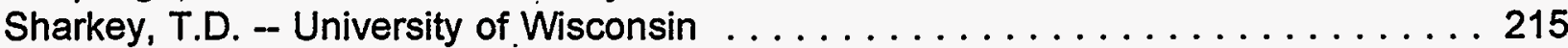

Sherman, L.A. -- Purdue University . . . . . . . . . . . . . . . . . . . . . 177

Silk, W.K. -- University of California - Davis $\ldots \ldots \ldots \ldots \ldots \ldots \ldots \ldots \ldots$

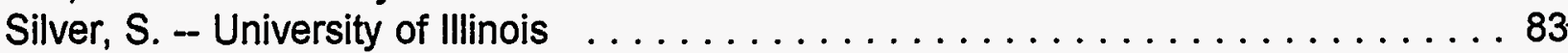

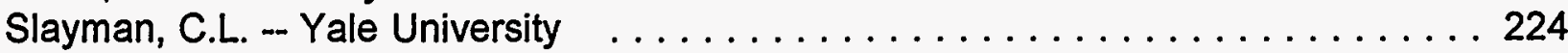

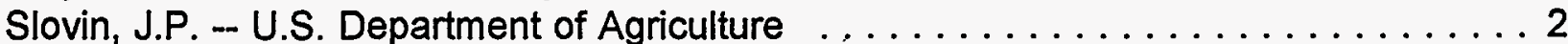

Söll, D. -- Yale University . . . . . . . . . . . . . . . . . . . . . . . . . 225

Somerville, C.R. -- Carnegie Institution of Washington $\ldots \ldots \ldots \ldots \ldots \ldots$

Somerville, S.C. -- Carnegie Institution of Washington . . . . . . . . . . . . 46

Sowers, K.R. -- University of Maryland . . . . . . . . . . . . . . . . . . . . . . 104

Stacey, G. -- University of Tennessee . . . . . . . . . . . . . . . . . . . . . . . . . . 194 
Staskawicz, B.J. -- University of California - Berkeley $\ldots \ldots \ldots \ldots \ldots \ldots 28$

Steponkus, P.L. -- Cornell University . . ..................... 59

Stern, D.B. -- Boyce Thompson Institute for Plant Research, Inc. . . . . . . . . . 16

Stevens, R. -- Lawrence Berkeley Laboratory . . . . . . . . . . . . . . . . . 97

Stewart, V.J. -- Cornell University . . . . . . . . . . . . . . . . . . 60

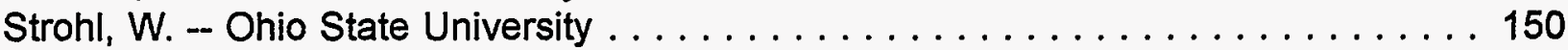

Sundaresan, V. -- Cold Spring Harbor Laboratory ................. 53

Sussman, M.R. -- University of Wisconsin $\ldots \ldots \ldots \ldots \ldots \ldots \ldots \ldots \ldots \ldots$

Sze, H. -- University of Maryland $\ldots \ldots \ldots \ldots \ldots \ldots \ldots \ldots \ldots \ldots \ldots \ldots \ldots \ldots \ldots$

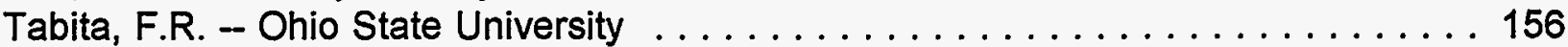

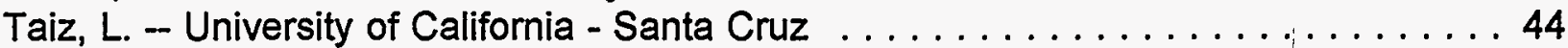

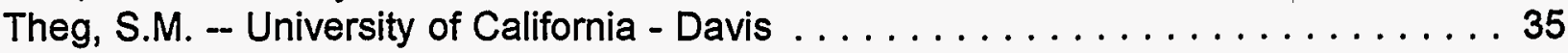

Tien, M. -- Pennsylvania State University $\ldots \ldots \ldots \ldots \ldots \ldots \ldots \ldots \ldots \ldots$

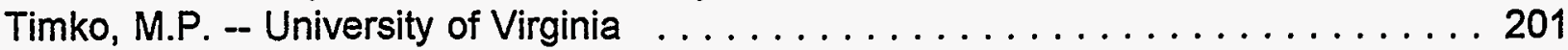

Tropper, F. -- Lawrence Berkeley Laboratory . . . . . . . . . . . . . . . . . . . 97

Unkefer, C.J. -- Los Alamos National Laboratory . . . . . . . . . . . . . . . 102

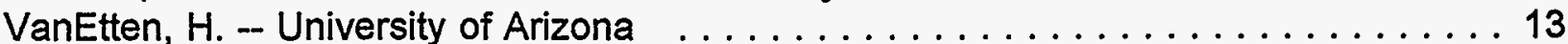

Venter, J.C. - The Institute for Genomic Research . . . . . . . . . . . . . . 199

Vermaas, W. -- Arizona State University . . . . . . . . . . . . . . . . 6

Vierling, E. -- University of Arizona $\ldots \ldots \ldots \ldots \ldots \ldots \ldots \ldots \ldots \ldots \ldots \ldots \ldots \ldots \ldots$

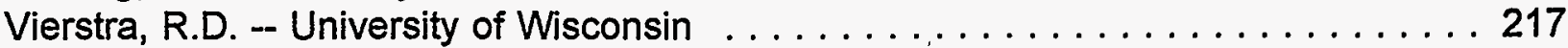

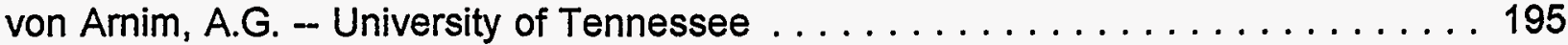

Voytas, D. -- lowa State University . . . . . . . . . . . . . . . . . 89

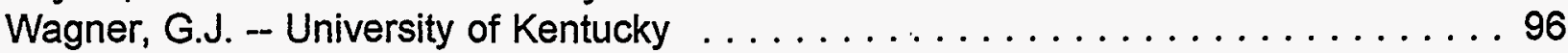

Wall, J.D. - University of Missouri $\ldots \ldots \ldots \ldots \ldots \ldots \ldots \ldots \ldots \ldots \ldots \ldots \ldots \ldots \ldots$

Walton, J.D. -- Michigan State University DOE Plant Research Laboratory . . . . 112,118

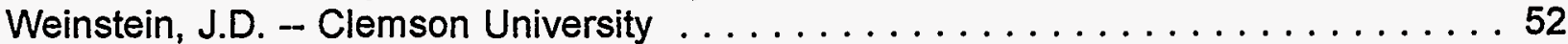

Wessler, S.R. -- University of Gecrgia $\ldots \ldots \ldots \ldots \ldots \ldots \ldots \ldots \ldots \ldots \ldots \ldots$

Whetten, R. - North Carolina State University . . . . . . . . . . . . . . . 145

Whitman, W.B. -- University of Georgia $\ldots \ldots \ldots \ldots \ldots \ldots \ldots \ldots \ldots \ldots$

Whitmarsh, J. -- University of Illinois $\ldots \ldots \ldots \ldots \ldots \ldots \ldots \ldots \ldots \ldots \ldots \ldots$

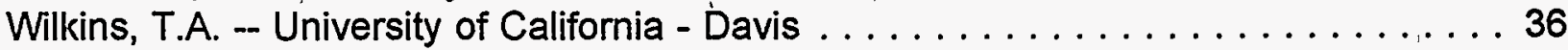

Wilson, D.B. -- Cornell University . . . . . . . . . . . . . . . . . . 61

Wolfe, R.S. -- University of Illinois . . . . . . . . . . . . . . . . . . 86

Wolk, C.P. -- Michigan State University DOE Plant Research Laboratory . . . . 119,120

Wong, C. H. - Lawrence Berkeley National Laboratory . . . . . . . . . . . . . . 97

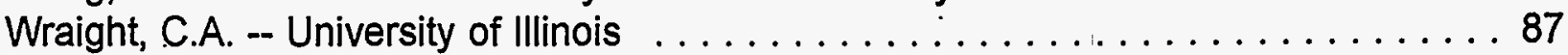

Wu, J.H.D. - University of Rochester . . . . . . . . . . . . . . . . 181

Yachandra, V.K. -- Lawrence Berkeley National Laboratory . . . . . . . . . . . 100

Yamamoto, H.Y. -- University of Hawaii . . . . . . . . . . . . . . . . 82

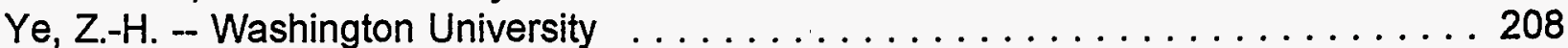

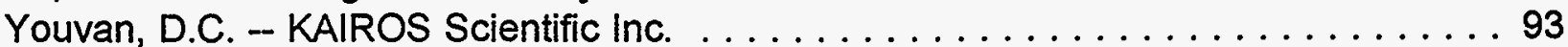

Zambryski, P. -- University of California - Berkeley . . . . . . . . . . . . . 29

Zeevaart, J.A.D. -- Michigan State University DOE Plant Research Laboratory . 114,121 
Zeiger, E. -- University of California - Los Angeles . ............... 42

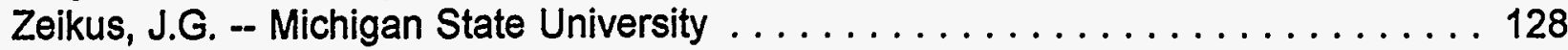

Zielinski, R.E. -- University of Illinois $\ldots \ldots \ldots \ldots \ldots \ldots \ldots \ldots \ldots \ldots \ldots$

Zinder, S.H. -- Cornell University $\ldots \ldots \ldots \ldots \ldots \ldots \ldots \ldots \ldots \ldots \ldots \ldots \ldots \ldots \ldots$ 